JUN231964

\author{
$L$ \\ CEA-1000 \\ March 1, 1964
}

\title{
THE CAMBRIDGE ELECTRON ACCELERATOR: \\ A COMPREHENSIVE ACCOUNT
}

Facsimile Price $\$$

Microfilm Price $\$$

Available from the Available from the Department of Commerce Washington 25, D. C.

PATENT CLEARANCE OBTAINED. RELEASE TO

THE PUBLIC IS APPROVED. PROCEDURES

ARE ON EILE IN THE RECEIVING SECTION,

CAMBRIDGE ELECTRON ACCELERATOR 


\section{DISCLAIMER}

This report was prepared as an account of work sponsored by an agency of the United States Government. Neither the United States Government nor any agency Thereof, nor any of their employees, makes any warranty, express or implied, or assumes any legal liability or responsibility for the accuracy, completeness, or usefulness of any information, apparatus, product, or process disclosed, or represents that its use would not infringe privately owned rights. Reference herein to any specific commercial product, process, or service by trade name, trademark, manufacturer, or otherwise does not necessarily constitute or imply its endorsement, recommendation, or favoring by the United States Government or any agency thereof. The views and opinions of authors expressed herein do not necessarily state or reflect those of the United States Government or any agency thereof. 


\section{DISCLAIMER}

Portions of this document may be illegible in electronic image products. Images are produced from the best available original document. 


\section{CAMBRIDGE ELECTRON ACCELERATOR}

Harvard University

42 Oxford Street

Cambridge, Massachusetts

CEA'-1000

March 1, 1964

\section{THE CAMBRIDGE ELECTRON ACCELERATOR: \\ A COMPREHENSIVE ACCOUNT}

Edited by W. A. Shurcliff

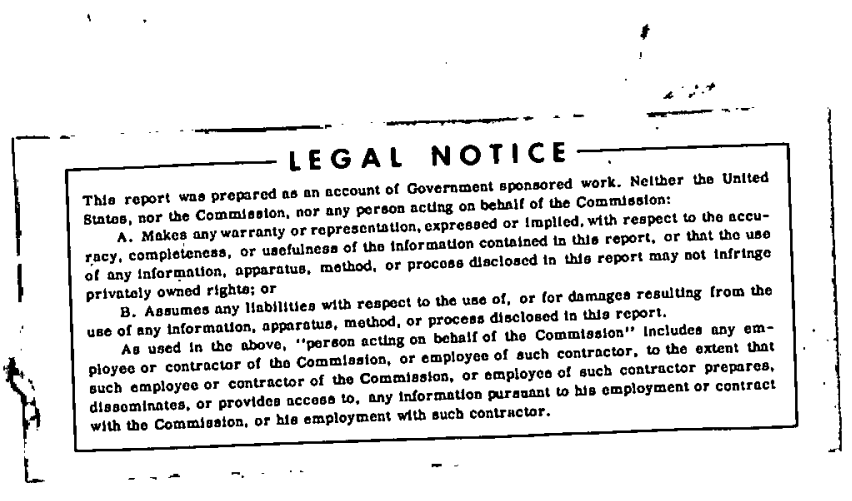




\section{Table of Contents}

Chapter

Preface

- Editor's note of acknowledgment

1 Origin of project

2

Administration

3

Basic plan

4

The 48 magnets

5

Supporting and aligning the 48 magnets

6

Powering the 48 magnets

7

Pole-face windings

8

Linac

Electron injection

RF system

Vacuum chambers

12

Vacuum pumps

13

Straight section tanks

14

Monitors of the orbiting beam

15

Coils for correcting orbit distortions at injection

16

Internal targets and target engagement means

17

External electron beam

Cabling and wiring

Control room

Shielding

Radiation monitoring equipment

Interlock system

Miscellaneous components of accelerator proper

Photon beams from machine targets

Synchrotron radiation

Cooling plant and water treatment plant

Tune-up and early operation

Magnets for use in experiments

Cryogenics

Miscellaneous facilities for experiments

Conclusions

Index 


\section{Preface}

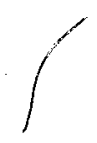

This account of the Cambridge Electron Accelerator was written in 1963 and early 1964, after more than a year of successful operation. It is the successor to Report CEA-81.

The present account summarizes the history, philosophy of design, actual design, construction, and early operation of this $6-\mathrm{Bev}$ electron synchrotron. In addition to describing each major component of the accelerator proper, it describes the various auxiliary facilities provided for use by experimentalists from nearby universities.

The account is intended to be useful to CEA scientists, engineers, and operations personnel, and also to visiting experimentalists. It may be of interest to accelerator designers generally. The account is not an operations manual or maintenance manual, and does not take the place of the detailed reports, manuals, engineering drawings, purchase specifications, etc., available. Also, it makes no attempt to describe the individual experiments that have been completed or are underway.

It is a pleasure to thank the U.S. Atomic Energy Commission for its continuing financial support throughout the seven-year period concerned, to thank the administrative officials of Harvard University for handling the fiscal and contractual affairs, and to thank the joint M.I.T.-Harvard Executive Committee for its wise guidance and unflagging encouragement. Finally, I wish to thank the CEA staff members for their skill in anticipating and overcoming all major difficulties and their hard work which permitted the accelerator to be completed and brought into operation promptly and effectively.

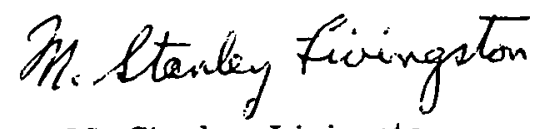

M. Stanley Livingston

Director 


\section{Editor's Note of Acknowledgment}

The writing of each portion of this report was dependent on extensive help from colleagues at CEA. Without their constant advice no adequate account could have been prepared. I am indebted to Dr. M. S. Livingston for general encouragement and counsel, and for preparing the chapter on the early history of the project. I am especially indebted to $\mathrm{K}$. W. Robinson for explaining to me, patiently and in detail, the rationale of the designs of the magnet ring, the pole faces, and the rf system, and for indicating the main factors governing the momentum compaction, betatron frequencies, amplitudes of betatron oscillations, beta function, damping, anti-damping, synchrotron oscillations, beam loading, orbit distortions, etc.

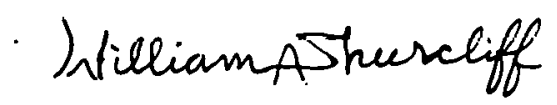

W. A. Shurcliff, Editor March 1, 1964. 


\section{Chapter 1}

\section{Origin of the Project}

1.1 1952: Earliest plans

1.2 1953: Proposal for a 15-Bev proton accelerator

1.3 1954: Switch to an electron accelerator

1.4 1955: Year of waiting

1.5 1956; The prnjest. is launched

\subsection{2: Earliest Plans}

The interest of M.I.T. and Harvard physicists in a high-energy accelerator for the Cambridge area preceded the invention of alternating gradient focusing by several years. At Harvard, it was clear even before completion of the 150-Mev synchrocyclotron in 1949 that the energy provided by this device was insufficient for the production of pions. The Harvard Nuclear Physics Committee, in deciding to complete this device as planned (rather than attempt to rebuild it for higher energy) did so with full recognition that a machine of considerably higher energy would be needed before long. During the spring of 1952 Prof. N. F. Ramsey initiated discussions as to the need for a higher-energy machine with the Department Visiting Committee (J. R. Oppenheimer, Chairman), which gave strong support. Ramsey informed officials of the Atomic Energy Commission of this interest and discussed with Profs. M. S. Livingston and J. R. Zacharias of M.I.T. the possibility of establishing a joint project.

At M.I.T. Livingston recognized that the time was ripe for developing a super-energy machine to attain energies well above the 2 to $3 \mathrm{Bev}$ then afforded by the Brookhaven Cosmotron and the soon-to-be-completed 6-Bev Bevatron at the Lawrence Radiation Laboratory at Berkeley. On May 14, 1952, Livingston presented a memorandum to Dr. J. A. Stratton, Vice President of M.I.T., proposing a design study for a large accelerator to be situated in Cambridge, hoping that members of the Brookhaven design group and also persons from other laboratories could be attracted to Cambridge.

Dr. L. J. Haworth, Director of the Brookhaven National Laboratory, was unable to release staff for a study at Cambridge, but invited Livingston to Brookhaven for the summer of 1952 to work with the Cosmotron. Cosmotron operation was delayed, and Livingston requested permission from Haworth to spend the summer, in collaboration with Brookhaven staff members, on a design study of a higher-energy accelerator. The importance of such 
a study was heightened by the impending visit to Brookhaven in August of a delegation of scientists from the newly-formed CERN Laboratory at Geneva, who were considering building a 10-Bev machine based on the Cosmotron or Bevatron designs. The Brookhaven study group started with the goal of designing a $10-\mathrm{Bev}$ machine that would take advantage of Cosmotron experience.

One of the first results of the 1952 summer design study was the conception and rapid development of the principle of alternating gradient (strong) focusing. This concept was exploited to form the basis of a preliminary design for an AG proton synchrotron, and calculations were made for synchrotron designs that would provide $10 \mathrm{Bev}, 25 \mathrm{Bev}$, $50 \mathrm{Bev}$, or even higher energies. By the time the CERN delegation arrived it was apparent that the new focusing concept could reduce the magnet and power costs sufficiently to justify plans for achieving energies far greater than $10 \mathrm{Bev}$. The CERN delegates were informed of the new ideas and were greatly impressed with the possibilities, leading :eventually to the 28-Bev CERN. AG proton synchrotron.

An account of the principle of strong focusing was published by E. D. Courant, M. S. Livingston and H. S. Snyder ${ }^{1}$ later that year, in a paper which described a possible 25-Bev AG synchrotron and also demonstrated the capabilities of quadrupole lenses for focusing linear beams. A companion paper by J. P. Blewett ${ }^{2}$ described the application to electrostatic gradient fields and the linear accelerator.

Prof. Ramsey came to Brookhaven in August, at which time it was clear that the AG principle offered a unique opportunity of crystallizing planning for a Cambridge accelerator. While at Brookhaven, Ramsey and Livingston prepared initial drafts of a proposal to the AEC. On returning to Cambridge in September they joined with Profs. J. C. Street of Harvard and J. R. Zacharias of M.I.T. as a self-constituted "Joint Accelerator Committee." One of the early study papers was a table of design parameters for a 10-Bev AGS prepared by Livingston on Sept. 24. An initial proposal for a 10-20 Bev Cambridge AG accelerator was sent by this committee on Oct. 10th to Dr. T. H. Johnson of the AEC. Following telephone exchanges with members of Dr. Johnson's office, this proposal was supplemented on Oct. 28th by a request for funds to support a design study at Cambridge. At a meeting at Brookhaven on Oct. 31 with representatives of the AEC and of the Brookhaven and Princeton planning groups, an agreement was reached to support the Cambridge study by means of a subcontract from Brookhaven to M.I.T. This decision was later confirmed by a letter subcontract dated Jan. 26, 1953. 
The "Cambridge Design Study Group" at M.I.T., under the direction of Prof. Livingston, started by holding a series of planning conferences on the several major components. Bulletin No. 1, dated Nov. 7, 1952, announced six conferences during the following week, and later bulletins kept the group informed. Local staff members participating in the study included: M. S. Livingston, N. F. Ramsey, E. M. Purcell, A. J. Poté, R. V. Pound, J. S. Clark, Lan Chu, R. A. Mack, J. C. Street, W. M. Preston, W. Selove, M. Wanagel, A. Wattenberg, and F. B. Robie. Others who joined in the design study during the spring of 1953 were: B. T. Feld, W. H. Bostick, R. Q. Twiss, B. J. Malenka, A. Abragam, R. J. Williams, J. A. Hofman, M. A. Levine, R. L. Smith, J. F. Fraser, P. Floyd, and Mrs. D. Slagel (secretary).

\subsection{3: Proposal for a 15-Bev Proton Accelerator}

An intensive effort by the Cambridge group during the spring of 1953 resulted in a report "Design Study for a 15-Bev Accelerator" (212 pp) completed June 30, 1953, and published as Technical Report No. 60 of the M.I.T. Laboratory for Nuclear Science. The report presented design calculations, a list of parameters, results of experimental magnet model studies, and a preliminary estimate of the cost of construction $(\$ 7,900,000)$. The site proposed in this early study was adjacent to the Harvard Cyclotron Laboratory, where the Cambridge Electron Accelerator now stands.

The report provided the technical basis for a proposal (submitted Aug. 8, 1953) to the AEC from the Joint Accelerator Committee for a 15-Bev AGS at Cambridge. A major obstacle was a policy recommendation by the General Advisory Committee of the AEC that multi-Bev accelerators should be located only in the national laboratories. However, the reduction in cost of multi-Bev machines provided by the AG principle justified reconsideration of this policy. The Cambridge Design Study subcontract from Brookhaven was extended, first to Sept. 1, 1953, and later to Sept. 1, 1954, when it terminated.

Meanwhile, design work at Brookhaven was aimed initially at an energy of $50 \mathrm{Bev}$; requests for support were submitted to the AEC. By late 1953 Brookhaven was informed that AEC funds available for such a large AGS machine were insufficient for $50 \mathrm{Bev}$, and the design energy was set at 25-30 Bev. By September, funds were authorized by the AEC for Brookhaven to design and build an electron "analog" (or model), to study the AG . focusing principle using electrostatic gradient fields, to determine precision requirements, and to evaluate the problems of crossing orbital resonance and the "transition" energy: 
By early 1954 Brookhaven plans for constructing the 30-Bev AGS were approved. On the other hand the AEC decided late in 1953 that it could not support at that time and at a university a project of the size proposed by the Cambridge group.

\subsection{4: Switch to an Electron Accelerator}

The approval of the 30-Bev AGS at Brookhaven and the disapproval of the Cambridge proposals required reconsideration of the plans in Cambridge. A few full-time members of the Design Study Group continued technical studies of AG magnet models and other components. But discussions in the scientific group shifted to consideration of an electron accelerator, also using AG magnets. Special problems such as the energy lost by the orbiting electrons through radiation, and the requirements for acceleration, were analyzed. The practical maximum energy was estimated to be 5 or $6 \mathrm{Bev}$, and early cost estimates suggested that such a machine would be more nearly "university size." A brief "Design Study for. a 6-Bev Electron Accelerator" was prepared by Livingston on March 15; a more complete study by the Design Study Group was prepared in May; further design analysis continued throughout the summer.

On June 10, the Harvard Physics Department voted unanimous approval of the plan for an electron accelerator. Support was obtained from the M.I.T. and Harvard administrations. On Aug. 17, 1954, the Joint Accelerator Committee, still consisting of Livingston, Ramsey, Zacharias and Street, submitted a proposal to the AEC for a "5-6 Bev Electron Accelerator." Efforts to obtain further funds to continue the design study through Brookhaven and the AEC failed, and the design subcontract was terminated on Sept. 1, 1954.

\subsection{5: Year of Waiting}

The Cambridge proposal gathered dust in Washington while problems of financial support for accelerators in universities vs. the national laboratories were being considered. Princeton submitted a proposal for a fast-cycling 3-Bev proton accelerator based on the Cosmotron. The MURA group sought support for an FFAG (fixed-field alternating-gradient) accelerator. The national laboratories at Argonne, Los Alamos, and Oak Ridge submitted plans for accelerators of various types. A "Memo for File" by Livingston on Jan. 31, 1955, describes the confused state of planning at that time.

During May and June of 1955 the Cambridge group requested interim support from the M.I.T. and Harvard administrations, to keep the design effort in being. On June 23, 1955, 
the two universities jointly provided a sum of $\$ 33,000$ to the "Cambridge Accelerator Project" - an amount sufficient to maintain a small staff and a small design laboratory. During this year Livingston continued as Director (part time) and many staff members of M.I.T. and Harvard contributed part-time services; three full-time members were recruited: K. W. Robinson, R. Waniek, and G. Lanza。

During the spring of 1955 , Congress somewhat unexpectedly included in the AEC appropriation bill an amount of $\$ 10,000,000$ for two university accelerators, presumably Lased un the proposals from Cambridge and Princeton. However, on July 6th Dr. T. H. Johnson sent a letter to the presidents of Harvard, M.I.T., and a number of other institutions asking for proposals for accelerators which might compete for this money, setting a maximum of $\$ 5,000,000$ for each and naming a closing date of October 1st. In response to requests from other institutions the decision date was postponed to Dec. 31st. Although the Cambridge group was disappointed at the delay, the time was used in preparing a more complete proposal for a 6-Bev accelerator - a printed proposal dated Dec. 15, 1955.

\subsection{6: The Project is Launched}

Still more months of delay intervened before a contract to support the Cambridge machine was awarded by the AEC. Meanwhile, local arrangements for insuring cooperation and fixing respunsibility were evolved, first by a letter from Dean McGeorge Bundy of Harvard to President Killian of M.I.T. on Jan. 5, 1956, and then by a March .7, 1956 "Memorandum of Agreement" signed by the presidents of the two institutions. Use of the present site near the Harvard Cyclotron was approved by the Harvard administration, and it was agreed that Harvard would act as the contracting agent with the AEC. The first letter-contract between Harvard and the AEC was signed and became effective on April 2, 1956. Contract negotiations extended for over a year, and the final definitive contract was signed on May 28, 1957. The effective date of April 2, 1956, was the official start of the design and construction of the Cambridge Electron Accelerator.

The original contract for construction of the CEA was for $\$ 6,500,000$. However, before this contract was signed it was known that this-amount would not provide sufficient laboratory space and sufficient facilities for full-scale operation. Accordingly a proposal for such additional facilities was submitted to the AEC on Aug. 13, 1956, and a contract extension agreement providing an additional $\$ 1,430,000$ was approved. As of May 28, 1957, the total estimated cost of construction was $\$ 7,930,000$. (As indicated in the next chapter, the final cost of construction was approximately $\$ 11,535,000$.) 
Main References: See reports, etc., mentioned above.

\section{Other References:}

1. E. D. Courant, M. S. Livingston, and H. S. Snyder, Phys. Rev. 88, 1190 (1952).

2. J. P. Blewett, Phys. Rev. $\underline{88}, 1197$ (1952). 
Chapter 2

Administration

2.1 Roles played by M.I.T. and Harvard

2.2 Executive Committee of the CEA

2.3 Scientific Subcommittee

2.4 Director

2.5 Staff

2.6 Support by the AEC

\subsection{Roles Played by M.I.T. and Harvard}

The Cambridge Electron Accelerator laboratory was created by Massachusetts Institute of Technology (M.I.T.) and Harvard. Basic policy questions as to planning, scope of activity, and scientific use of the accelerator have been settled,jointly by these two universities. For the purpose, the Executive Committee of the CEA, described in a later paragraph, was created.

Fiscal and legal negotiations with the U. S. Atomic Energy Commission (AEC), which provided the funds for constructing and operating the accelerator, have been handled by Harvard, through its Office for Research Contracts. Other administrative matters dealt with by Harvard have been: employment, issuing of paychecks to employees, general policing and fire protection, supply of film badges, provision of a parking area, and, of. course, making available the tract of land on which the accelerator was built.

\subsection{Executive Committee of the CEA}

Basic policies concerning the construction and use of the accelerator have been established by a ten-man Executive Committee of the CEA, consisting of five representatives of Massachusetts Institute of Technology and five representatives of Harvard University. Each group of five includes two administrators and three tenure scientists. The Committee meets once or twice a year and informal discussions are held at other times. The membership has changed from time to time, and has included, for various periods, the persons indicated below. Asterisks denote persons who were members on March 1, 1964.

From M.I.T.: Adm. Edward L. Cochrane (admin.)

Dr. Peter T. Demos (admin.)

Dr. Martin Deutsch *

Dr. Bernard T. Feld * 


Dr. Carl F. Floe * (admin.)
Dr. Nathaniel H. Frank (admin.)
Dr. David H. Frisch
Dr. James McCormack (admin.)
Dr. Louis S. Osborne *
Dr. Charles H. Townes * (admin.)
From Harvard:
Dr. McGeorge Bundy (admin.)
Dr. Franklin L. Ford * (admin.)
Dr. Edward M. Purcell
Dr. Nathan M. Pusey (admin.)
Dr. Norman F. Ramsey *
Mr. Edward Reynolds (admin.)
Dr. Karl Strauch
Dr. J. Curry Street *
Mr. L. Gard Wiggins * (admin.)
Dr. Richard Wilson*

On June 6, 1963, Dr. Charles H. Townes of M.I.T. became Chairman.

\subsection{Scientific Subcommittee}

The six tenure-scientists on the Executive Committee comprise the Scientific Subcommittee, which meets monthly to discuss specific scientific or technical issues with the Director.

Assisting this Subcommittee is a "Planning Committee for the CEA," established in 1962 for the purpose of working out the optimum scheduling of the accelerator for use by whatever groups of experimentalists are ready to make runs. The Planning Committee also makes recommendations to the Director as to the support facilities to be provided to the. experimentalists by the CEA. Until June 1963 the M.I.T. and Harvard members of this Committee were M. Deutsch and R. Wilson. Starting in July 1963 the members were L. Osborne and F. Pipkin.

\subsection{Director}

From the outset Professor M. Stanley Livingston of M.I.T. has been Director of the CEA. From Sept. 1962 until May 1963 he was on leave of absence and Professor J. Curry Street of Harvard served as Acting Director.

In January $1959^{\circ} \mathrm{Dr}$. Thomas L. Collins was made Assistant Director, and in June $1960 \mathrm{Mr}$. Robert E. Cummings was made Assistant Director. 
$2.5 \quad$ Staff

The staff increased in number as construction advanced and as the number of experiments in progress or in preparation increased. By August 1960 there were 100 employees and by September 1963 there were 156.

The largest administrative subdivisions of the CEA organization during the construction period were the Mechanical Engineering Division, Electrical Engineering Division, Electronics Division, and machine shop group. The Operations Division was established on March 14, 1962, when the synchrotron was being put into operaliun, and soon became the largest of the divisions.

The following list includes the names of most of the principal scientific and engineering persons engaged - in the past or at present (March 1, 1964) - in design, construction, operation, or administration. Also, it indicates the main interests and responsibilities of the persons concerned.

R. J. Averill: magnet power supply; operations; beam-bump method of target engagement. W. E. Balch: business administration.

Dr. A. E. Barrington (Ex): linac.

B. Berkes (Ex): magnet measurements, magnetic shielding.

H. Biggelaar: electrical power.

R. D. Biron: mechanical engineering, cryogenicэ.

A. Brambilla: electrical facilities.

F. W. Brasse (Ex): ejection theory.

F. P. Brooks: mechanical design, cryogenics.

P. H. Burr: mechanical design.

A. J. Carr: power supplies.

Dr. T. L. Collins: Assistant Director; system coordination; orbit theory; vacuum system, magnets, controls, tune-up, beam-bump method of target engagement, external photon beam, external positron beam, Experimental Hall allocations, buildings and grounds.

Dr. P. F: Cooper, Jr.: magnet design.

R. E. Cummings: Assistant Director for Administration.

Dr. J. Dekleva (Ex): rf models and system design.

Dr. J. K. de Pagter: magnet measurements, correction coils, shielding, ionization chambers, Faraday cup, quantameters.

Dr. D. S. Edmonds (Ex): vacuum pump power supplies. 
Dr. R. Fessel: pole face windings, coordination of experimental arrangements, special detectors, beam analysis, magnet evaluation.

Dr. G. E. Fischer: rf waveguides, ejection of external electron beam, experimental facilities, special detectors.

J. Fitzgerald: electronic instrumentation.

Dr. M. Fotino: special vacuum chambers, quantameters, shielding measurements.

G. Gonzalez: electrical facilities, interlock systems.

H. J. Hajko (Ex): electronics, data processing.

Roger Hall (Ex): mechanical engineering.

Dr. V. Henri (Ex): magnet measurements.

W. S. Henry: cabling, power supply.

J. Hess (Ex): vacuum pumps and vacuum measurement.

H. L. Holcomb: electrical circuits and electronic instrumentation.

G. L. Homsy: electrical power.

Dr. D. D. Jacobus: mechanical engineering; design of major mechanical components; layout of magnet ring; vacuum chamber design; magnet design.

W. J. Jones: electrical facilities, electrical systems planning, cabling, controls, power supplies, electrical safety.

S. C. Kao (Ex): radiation monitoring, operations.

A. Kluge (Ex): mechanical design.

H. Kumpfert (Ex): radiof requency problems.

Dr. G. Lanza (Ex): orbit theory.

L. A. Law: electronics, electronics system planning, instrumentation, beam-bump switching.

L. Leonard (Ex): linac testing.

Prof. M. S. Livingston: Director.

Dr. R. A. Mack: vacuum system; gas analysis; radiof requency problems; circuit analysis; interlock system.

B. J. Maddox: electronics, radiation monitors, instrument design, operation.

Dr. H. R. Myers (Ex): electronic instrumentation, radiation monitoring systems design, special monitors, interlock system.

G. L. Nicholls: radiof requency system, operations.

Dr. H. Nysater (Ex): magnetic measurements.

Dr. J. M. Paterson: orbit distortions, external electron beam.

D. I. Porat (Ex): electronics, electronic systems planning, beam-displacement pick-up coils, intensity monitors, controls.

M. Ramage: magnet appraisal, electrical equipment. 
Dr. J. R. Rees: radiofrequency system planning, components, and power supplies; beam loading problems.

L. C. Robinson (Ex): radiofrequency system, betatron oscillation analysis, linac testing.

K. W. Robinson: system coordination, orbit theory, focusing theory, magnet ring design, radiofrequency system design, magnet alignment analysis, beam loading analysis, photon beams, external electron beam, injection systems, synchrotron radiation.

B. Roscoe (Ex): purchasing.

R. I. Samuel: electronics.

V. Scheurkogel (Ex): mechanical engineering.

Dr. R. Sherwood: inflection, momentum matching system.

Dr. W. A. Shurcliff: magnet alignment, radiation safety, technical reports.

Dr. J. A. Smith (Ex): cryogenics.

Dr. R. Smith (Ex): detection equipment, Faraday cups, linac testing.

Dr. W. I. B. Smith (Ex): injection optics, circuit analysis.

Dr. H. S. Snyder (Ex): theoretical studies.

Dr. K. Steffen (Ex): radiofrequency system, injection optics.

Dr. J. C. Street (Ex): Acting Director.

B. K. Vetter: electrical engineering, operations.

Dr. G. A. Voss: linac testing, inflection, tune-up, operations planning, radiation safety.

C. Wakstein (Ex): mechanical design, shielding.

Dr. R. W. Waniek (Ex): magnet model studies.

J. A. Weinstein: electronic instrumentation.

Dr. M. Widgoff (Ex): reports.

Dr. H. Winick: magnet measurement, pole face windings, vacuum system, tune-up, operations.

C. W. Wooldredge: purchasing.

Dr. L. A. Young (Ex): magnet power supplies, magnet design, special power supplies.

\subsection{Support by the AEC}

The accelerator was designed and built under Contract AT(30-1)-1909 between

Harvard University and the U.S. Atomic Energy Commission. The contract became effective April 2, 1956. The original amount of the contract was $\$ 6.5$ million, but the amount was soon increased to $\$ 7.93$ million to provide laboratory space and facilities for full-scale operation. As construction proceeded other additions and revisions were made; they brought the total to $\$ 11.535$ million. The contract terminated on March 30,1962 . 
Support for research operations has been provided by an operations contract, AT(30-1)-2076, which became effective April 1, 1958, and thus overlapped the construction contract discussed above. Each year, additional funds have been added to the operations contract to cover the year's operations. Prior to 1962 most expenditures charged to this contract were for auxiliary facilities such as special magnets required in various experiments, power supplies for these magnets, a large cryogenics plant, an adequate inventory of shielding blocks, radiation monitoring equipment, etc. After the construction contract was terminated, all laboratory costs were charged to the operations contract. Each year the CEA furnishes the AEC with an estimate of the funds needed for the subsequent fiscal year.

The university lahoratories from which the scientific users of CEA come are supported by separate AEC contracts; these provide the special-purpose equipment required in the individual experiments at the CEA. In general, the CEA provides basic equipments and services essential to a sequence of experiments and the various universities provide the apparatus (including data-analysis systems) specific to individual experiments. 


\title{
Chapter 3
}

\section{Basic Plan}

\author{
3.1 Introduction \\ 3.2 Main design parameters \\ 3.3 The controlling considerations \\ 3.4 Expected operation \\ 3.5 Effectiveness of alternating gradient focusing \\ 3.6 Phase stability \\ Appendix: Some basic definitions
}

\section{$3.1 \cdot$ Introduction}

When, in 1954, physicists participating in the Cambridge Accelerator Project decided to concentrate their efforts on the design of a multi-Bev strong-focusing electron synchrotron, many grand questions of policy confronted them. To arrive at the best basic plan, they had to make two kinds of compromises: compromises between technical goals that to some extent were mutually exclusive, and compromises between what appeared technically optimum and what appeared economically acceptable. None of these compromises could be made without weighing the various goals, exploring the various technical means of achieving the goals, and preparing estimates of costs in terms of dollars and also years. Added difficulties were the interplay among the various key parameters, uncertainties as to some of the more daring and attractive avenues, and occasional changes of requirements by the prospective users of the accelerator.

\subsection{Main Design P.arameters}

The design finally adopted called for the following:

An alternating-gradient electron synchrotron providing a maximum energy of 6 Bev. A location on Harvard University grounds; specifically a location adjacent to the Harvard Cyclotron Laboratory.

A set of 48 magnets in a ring $118 \mathrm{ft}$. in radius; hence an -orbital frequency of 1.321 megacycles/sec and an orbital period of $0.757 \mu$ s.

A FODO magnet lattice with horizontally focușing magnets $F$ and defocusing magnets D separated by equal-length free-field regions $O$.

A magnetic field gradient that would result in betatron oscillation frequencies of 6.4 per turn. 
A repetition rate of 60 cycles per second.

Fully biased waveform of 60-cycle magnet excitation and a power supply employing a resonant circuit of inductors and capacitors.

A 20-to-30 Mev linac operating at 2855 megacycles/sec for injecting one pulse of electrons into the ring each cycle.

A pulsed magnetic inflector.

A set of 16 symmetrically located, high-Q, $\mathrm{rf}$ acceleration cavities, each including two chambers.

An acceleration frequency of 476 megacycles/sec, which is $1 / 6$ of the linac frequency and 360 times the orbital frequency.

A set of 48 vacuum chambers having transversely slotted metallic walls and vacuum-tight, dielectric (non-conducting) wrappings.

A set of 48 high-vacuum pumps of the titanium-discharge type.

An underground Circular Tunnel for housing the accelerator proper.

A widened portion ("Target Area") of the Circular Tunnel to accommodate beam-handling equipment such as collimators.

A large Experimental Hall adjacent to the Target Area and separated from it by a Main Shielding Wall in which six beam channels are provided.

A central Power Building for housing the principal power supplies, cooling system, and controls.

An adjacent Laboratory Building and (in 1964) a special room for housing electronic equipment used in data handling.

The nominal, or design, intensity figure adopted was $1 \times 10^{11}$ electrons per pulse, or $6 \times 10^{12}$ per second.

\subsection{The Controlling Considerations}

Some of the principal governing factors are considered below in a sequence that is somewhat arbitrary.

Location; Radius; Orbital Frequency: The first decision was that the synchrotron should be built on university property in Cambridge, Mass., so as to be an integral part of the physics research facilities of M.I.T. and Harvard. The only large plot of land available was a 275-ft-wide tract adjacent to Oxford St., close to the Harvard physics and 
engineering buildings and adjacent to the Harvard synchrocyclotron. As indicated by Fig. 3.1, the size of the plot imposed an upper limit on the radius of the magnet ring (about 115 or $120 \mathrm{ft}$.). The exact choice was made later, after the frequency of $\mathrm{rf}$ power had been decided; as explained on a later page the eventual choice of radius was $118 \mathrm{ft}$., and the resulting orbital frequency of a particle traveling at approximately the speed of light was $1.322 \times 10^{6}$ per second. The decision was made that the orbit should be at grade 21.5 relative to Cambridge Base Grade and about $10 \mathrm{ft}$. below local ground level. (Cambridge Base Grade is said to be $5 \mathrm{ft}$. below mean low water.) Also, it was decided that the floor level of the circular tunnel and Experimental Hall should be $5 \mathrm{ft}$. below orbit level, i.e., at grade $16.5 \mathrm{ft}$.

Repetition Rate: A high repetition rate of magnet excitation seemed desirable for several reasons: greater quantities of accelerated electrons would be produced per hour for use by experimentalists; the loss of energy by synchrotron radiation would be smaller, per electron; the time interval in which emission of synchrotron radiation could cause radial blow-up of the beam would be shorter and hence fewer very-high-energy electrons would be lost from the beam.

A high repetition rate was achievable if eddy current losses in the magnets could be avoided, as by constructing the core blocks of thin, electrically insulated laminations, and if an efficienl syslem could be devised for storing the magnet excitation energy during the intervals in which the excitation was small. The designers found that a repetition rate of 60 cycles per second could be achieved by making the core blocks of $0.014^{\mathrm{n}}$-thick laminations and by providing an energy storage system employing a large capacitor bank and a special 60 -ton inductor.

Number of Magnets; Length of Magnet; Length of Straight Section: The number of magnets used must be large enough (say 35 or more) so that the individual magnet is short compared to a quarter of a betatron wavelength, as explained in Chap. 4. Otherwise, the amplitudes of betatron oscillations would tend to be unduly large and hence the acceptance ellipse of the magnet system would be unduly small. The decision was made to use 48 magnets, since this number affords various symmetric sets of locations for $\mathrm{rf}$ cavities, beam displacement sensors, etc. For example, rf cavities may be placed at.16 symmetric locations: ; was also decided that the individual magnet should have a net length of $11 \frac{1}{2} \mathrm{ft}$. (implying an overall length of about $13 \mathrm{ft}$.) so that the intervening straight sections would be long enough (about $3 \mathrm{ft}$.) to accommodate rf cavities (see a later paragraph) and long enough to allow for convenient ejection of electron beams. 
Maximum Energy of Accelerated Electrons: If electrons are to be accelerated to $6 \mathrm{Bev}$ energy in a 118-ft-radius circle comprising 48 magnets of the above-indicated length, the vertical magnetic field in the magnet gaps must have a maximum value of at least 7600 gauss. This is well below the saturation value of better types of transformer steel; consequently a 6-Bev energy seemed feasible from the standpoint of magnet design. But would the requirements as to $\mathrm{rf}$ accelerating potentials and $\mathrm{rf}$ power be acceptable? It was well known that high-energy electrons traveling in curved paths emit synchrotron radiation, consisting largely of soft X-rays and ultraviolet radiation. A formula presented in Chap. 10 shows that the power loss by synchrotron radiation increases with the fourth power of the electron energy. Calculations showed that the energy lost by a 6 Bev electron traveling in a 118-ft-radius magnet ring would amount to $4.5 \mathrm{Mev}$ per turn around the ring. Thus the question facing the designers was: Can one design an rf system that will supply not only enough power to make up for the radiation loss but also to increase. the electron's energy at a reasonably rapid rate, such as $1 \mathrm{Mev}$ per turn? They found the answer to be in the affirmative, provided that the $\mathrm{rf}$ power is to be delivered by a large number of resonant chambers (for example, 16 double-chamber cavities) and that the cavities can be made short enough (by choosing an $\mathrm{rf}$ frequency high enough) so as to fit in the 3-ft. straight sections.

Power Requirements for Magnet Excitation and for the rf Cavities: If 60 groups of 6-Bev electrons were to be produced each second, the power needed for magnet excitation would amount to about $1 \mathrm{Mw}$. and the average amount of $\mathrm{rf}$ power needed would be about $100 \mathrm{kw}$. The peak value of $\mathrm{rf}$ power needed (assuming a reasonable choice of synchronous phase angle discussed in a later section) would be about 300 or $400 \mathrm{kw}$. It appeared likely that the goals could be met.

Choice of Frequency of $\mathrm{rf}$ Power: It was obvious at the outset that the radiofrequency must be high. For reasons indicated elsewhere it was apparent that the rf power. requirement would be large, and to minimize it the frequency must be high enough to permit choice of an rf cavity design affording high shunt resistance, hence high efficiency of power utilization. High frequency, i.e., high harmonic number, was essential also to keeping the amplitudes of synchrotron oscillations within reasonable limits at injection; a low radiofrequency would permit large radial amplitudes, and the result would be that an unnecessarily large fraction of the electrons would soon be lost from the beam (unless the cross-sections of the magnet gaps and vacuum chambers were increased). 
After the designers had decided to install, in the 16 uniformly spaced straight sections, cavities comprising two chambers operating in $\pi$ mode (i.e., in opposite phase), the choice of radiofrequency was narrowed further by the need to have the voltage in the second chamber just reverse in the time taken for the electron to travel from the center of the first chamber to the center of the second. If the center-to-center spacing of the chambers was to be in the neighborhood of $1 \mathrm{ft}$. $(30 \mathrm{~cm}$.), a radiofrequency in the range from 450 to $550 \mathrm{Mc} / \mathrm{sec}$ was required.

The choice was narrowed still further by the requirement that an orbiting electron encounter the same phase of voltage in a given cavity on successive turns around the ring. Thus the radiofrequency must be a harmonic of the orbital frequency $f_{0}$, already fixed at about $1.3 \times 10^{6}$ per second. When the decision was made that each of the 16 regularly spaced cavities should operate in opposite phase from its neighbor, the requirement on the $\mathrm{rf}$ became still more stringent: it must be an odd harmonic of $\left(8 \mathrm{f}_{0}\right)$. For example, it must be chosen from the set: $344 \mathrm{f}_{0}, 352 \mathrm{f}_{0}, 360 \mathrm{f}_{0}$, etc.

Another requirement on the choice of $\mathrm{rf}$ stemmed from the policy decision that, eventually, the radiofrequency should be tied to the linac frequency (already fixed at 2855. Mc/sec) in some integral relationship. Such a relationship would permit modulation of the linac beam at exactly the frequency of the main $\mathrm{rf}$ system and with whatever phase maximizes the fraction of freshly injected electrons that are captured into stable orbits, as explained in a later chapter.

The decision was made to use a radiofrequency that was just $1 / 6$ of the linac frequency, i.e.; a radiofrequency of about $476 \mathrm{Mc} / \mathrm{sec}$. (The exact figure adopted initially was $475.83 \mathrm{Mc} / \mathrm{sec}$, but a slight change was made later, as explained in Chap. 10.) By setting the accelerator radius at $118 \mathrm{ft}$. and arriving at an orbital frequency $\mathrm{f}_{\mathrm{o}}$ of $1.32 \times 10^{6}$. per sec, the designers arrived at the happy situation in which the rf was the 360 th harmonic of $f_{0}$ and the 45th harmonic of $8 f_{0^{-}}$Cavities having excellent shunt resistance could be designed and the amplitudes. of synchrotron oscillation could be kept small. Power tubes operating at $476 \mathrm{Mc} / \mathrm{sec}$ and having sufficiently high peak power rating and average power rating could be procured without undue delay.

As explained in a later chapter, the actual frequency used late in 1963 and early in 1964 was slightly lower, and in addition a small amount of frequency modulation was employed at the start of the cycle. 
The justifications for certain other basic choices, such as the "FODO" sequence of. magnets, a pole-face shape leading to betatron oscillation frequencies of 6.4 per turn, and a linac energy of 20 to $30 \mathrm{Mev}$, are indicated in later chapters.

\subsection{Expected Operation}

The expectation was that the synchrotron would operate as follows: Electrons from an oxide-coated cathode at the upstream (source) end of the linac are accelerated to an energy of about 20 or $25 \mathrm{Mev}$ and a speed of about $0.9997 \mathrm{c}$ or $0.9998 \mathrm{c}$. They are then focused and guided toward Straight Section 1 in the circular tunnel, where an inflection device causes them to join the circular orbit smoothly. Having an appreciable spread of momentum and direction, the stream of electrons more than fills the cross-section of the useful region of the magnet-gap; each electron executes horizontal and vertical betatron oscillations, about the equilibrium orbit appropriate to its energy and executes longitudinal (synchrotron) oscillations also. Those electrons that execute very-large-amplitude oscillations trespass outside the region of proper field and field gradient; consequently they oscillate with rapidly growing amplitude and soon strike the wall of the vacuum chamber and are lost from the beam. Electrons that execute small oscillations are captured into stable orbits and are repeatedly accelerated as they pass through the successive rf cavities.

The field strength in the magnet gaps increases smoothly and the.energy of the electrons increases correspondingly. At the end of about 8 milliseconds the magnetic field ceases increasing and the acceleration process comes to an end. By this time the electrons have traveled around the ring about 10,000 times, have gone a distance of 1400 miles, have acquired an energy of $6 \mathrm{Bev}$, a momentum of $6 \mathrm{Bev} / \mathrm{c}$, a mass 12,000 times the rest mass, and a speed of $0.999,999,996 \mathrm{c}$. The magnetic field then decreases smoothly to approximately zero (in about 8 milliseconds), and the cycle then repeats, 60 times a second.

Throughout the acceleration interval the amplitude of vertical betatron oscillation of a given electron tends to decrease, i.e., tends to be damped. The major part of the decrease (the so-called adiabatic damping) may be explained in somewhat popular and imprecise language by saying that the mass of the electron increases and accordingly if the energy of oscillation remains approximately constant the amplitude must decrease. A more precise explanation is that the magnitudes of field and field gradient in the magnet gaps are increasing steadily, and the electromagnetic fields in the rf cavities produce a proportionate increase in the forward-directed component of the momentum of the electron 
but do not increase the transverse component; therefore the amplitude of the transverse oscillation decreases. During the latter part of the acceleration interval the amount of synchrotron radiation emitted becomes large enough to be significant and contributes to the damping; the magnitude of this contribution (i.e., the radiation damping) can be computed using classical radiation theory; that is, the quantum nature of the radiation may be disregarded. During 6-Bev operation of the synchrotron, the height of the beam is presumed to be perhaps 10 or $20 \mathrm{~mm}$ at injection, $1 \mathrm{~mm}$ halfway through the acceleration interval, and $1 / 4$ to $1 / 2 \mathrm{~mm}$ at the end of this interval. During $3-\mathrm{Bev}$ operation the corresponding figures are 10 or $20 \mathrm{~mm}, 2 \mathrm{~mm}$, and $1 \mathrm{~mm}$ respectively.

The horizontal betatron oscillations, likewise, tend to decrease in amplitude during the first part of the acceleration interval. The cause of the decrease is adiabatic damping, and the magnitude of this damping is approximately equal to that of the vertical oscillations. However, during the latter part of the acceleration interval (i.e., after the electron energy reaches 3 or $4 \mathrm{Bev}$ ) the amplitude of the horizontal betatron oscillation tends to increase as a consequence of the emission of synchrotron radiation. The amplitude increases, rather than decreases, for the following reasons: the total amount of radiation emitted is large, it is emitted in the form of large (e.g., 0.1 to $10 \mathrm{kev}$ ) quanta, the quanta are emitted in the horizontal plane, and - most important - they are emitted preferentially at those times when the curvature of the eleclrun path is greatest, i.e., when the electron is farthest outward from the equilibrium orbit. The significance of this last circumstance stems from the fact that when an electron loses momentum, its equilibrium orbit is shifted inward, and consequently the electron finds itself farther from the equilibrium orbit. Accordingly, in succeeding oscillations the electron follows an even more strongly curving path, and the tendency for it to emit additional quanta "at just the wrong momeint" is accentuated. As a consequence of this radiation anti-damping, the amplitude of the horizontal betatron oscillation grows approximately exponentially. Fortunately the interval in which the anti-damping occurs is so short (about 3 or 4 milliseconds) that only a negligible fraction of the electrons are lost from the beam during this stage. During 6-Bev operation of the synchrotron the width of the beam is presumed to be about $40 \mathrm{~mm}$ at injection, 3 to $4 \mathrm{~mm}$ halfway through the acceleration interval, and 8 to $12 \mathrm{~mm}$ at the end of the interval.

At the start of the acceleration interval a modest level of $\mathrm{rf}$ power suffices. But near the end of the interval the power level is increased sharply to provide the electrons with sufficient energy to make up for the large losses by synchrotron radiation. Further details as to rf power are presented in Section 3.6 and Chap. 10. 
It was expected that about $10^{11}$ electrons would survive for the full acceleration interval. Thus $6 \times 10^{12}$ fully accelerated electrons would be produced each second. (When $10^{11}$ electrons travel around the orbit at the rate of $1.322 \times 10^{6}$ turns/sec, the "average circulating current" is 21.0 milliamperes. The "delivery" of $6 \times 10^{12}$ electrons per second corresponds to an average current of 0.96 microampere, and if the electron energy is $6 \mathrm{Bev}$, the average power delivered is nearly $6 \mathrm{kw}$.)

It was expected that, at the end of the acceleration interval, the electrons would be used in either of two ways: (1) They could be made to strike a small target situated inside the vacuum system and only an inch or two from the equilibrium orbit; a very slender beam of multi-Bev photons (bremsstrahlung) would result; these could be allowed to pass through a hole in the Main Shielding Wall to be available for use in the Experimental Hall. (2) They could be jolted out of orbit by a small, pulsed magnet; thus they would constitute an external beam that could be focused and aimed in any required way by means of d.c.-powered magnets.

The orbiting electrons travel, of course, in a vacuum. They travel in a $750-\mathrm{ft}-$ circumference "vacuum ring" consisting of 48 long vacuum chambers, $16 \mathrm{rf}$ cavities, and 32 straight section tanks. The vacuum chamber, resembling a partially flattened tube, consists mainly of stainless steel, and is evacuated to a pressure of about $10^{-6}$ torr by means of high-vacuum pumps situated beneath the straight sections.

\subsection{Effectiveness of Alternating Gradient Focusing}

The transverse deviations of an orbiting electron from its equilibrium orbit remain within acceptable bounds because of the focusing action provided by the two types of magnets, i.e., by the alternating gradients. Since horizontal oscillations have greater amplitude in the closed magnets than in the open magnets, as suggested by Fig. 3.2 , and since the horizontal restoring force is approximately proportional to the horizontal displacement, the horizontal restoring forces exerted by the closed magnets outweigh the horizontal defocusing forces exerted by the open magnets. Thus the net effect is a positive horizontal focusing effect. The vertical oscillations have greater amplitude in the open magnets than in the closed ones; hence the vertical focusing force of the former outweighs the defocusing force of the latter, and the net effect is, again, positive focusing. In summary, positive focusing occurs for oscillations having any transverse direction. (The alternating gradient focusing principle-is discussed in greater detail in the following chapter. It will be noted 
that the symbol $z$ is used to denote the vertical displacement of an orbiting electron from the horizontal median plane of the magnet gap and the symbol $x$ is used to denote the horizontal displacement from the vertical plane that contains the isomagnetic line of the magnet. The symbols $\nu_{\mathrm{z}}$ and $\nu_{\mathrm{x}}$ denote the frequencies of vertical and horizontal betatron oscillations in terms of cycles of oscillation per turn around the orbit.)

The calculated values of maximum and minimum beta function (defined in the appendix to this chapter) of horizontal betatron oscillation are $600^{n}$ and $100^{n}$. Thus the maximum and minimum values of half-width of the band swepl (see Fig. 3.1) by an electron that makes an angle of 0.001 radian with the equilibrium orbit when it crosses this orbit at the center of a closed magnet are $(0.001) \sqrt{(600)(600)}$ and $(0.001) \sqrt{(100)(600)}$, or about $0.60^{\prime \prime}$ and $0.24^{n}$ respectively. The maximum and minimum values of beta function for the vertical betatron oscillations are approximately the same: $600^{n}$ and $100^{\mathrm{n}}$; the maximum occurs, of course, in the open magnet. Graphs of representative betatron oscillations are presented in CEA-90.

The calculated values of "displacement component of momentum vector of the equilibrium orbit" (defined in the appendix to this chapter) are $57^{n}, 29^{n}$, and $42^{n}$ for the center of a closed magnet, center of an open magnet, and center of a straight section. The average value (i.e., value obtained by averaging with respect to distance $s$ along the ideal orbit) is $42^{n}$. The calculated value of average momentum compaction factor, also defined in the appendix, is $42 " / 140{ }^{n}$, or 0.03 .

The shape of the equilibrium orbit segment that lies within the gap of the upstream half of a closed magnet conforms closely (but not exactly) to an arc of a circle of about $86 \mathrm{ft}$. radius; see Fig. 3.3. If the electron has an energy of $6 \mathrm{Bev}$ and the $\mathrm{rf}$ cavities are powered at the design frequency, the position of the segment is such that the included areas radially outward and radially inward from the isomagnetic line are nearly equal. The sum $\left(d_{1}+d_{2}\right)$ of the outward deviation at the center of the half-magnet and the inward deviation at the outer end of an outermost core-block is $0.47 "$. The same is true; of course, for the other half of the magnet. For the open magnet the situation is much the same, although here the sum $\left(d_{1}+d_{2}\right)$ is greater by about $0.005^{n}$, and is thus about $0.48^{n}$. Actually, in a closed magnet the distance $\mathrm{L}$ of the equilibrium orbit from the vertical plane in which the extrapolated strength of the magnetic field is zero varies approximately as the cosine of the following function: some constant $\mathrm{C}_{1}$ times the distance $s$ along the magnet ( $s$ being measured from the center of the half-magnet). In an open magnet 
it varies approximately as the hyperbolic cosine of this same function. But the two curves are nearly alike: they lie on either side of the circular arc mentioned and they depart from it by only a few mils. The value of $\mathrm{C}_{1}$ is such that the argument of the cosine has the value $18^{\circ}$ at the outer end of an outermost core-block.

In a straight-section tank the nominal position of the equilibrium orbit is the tank centerline, defined in a later chapter. Between the magnet and the tank, however, there is a region of "magnet end extension," and the equilibrium orbit has, of course, appreciable curvature here.

The "phase angle length" $\Delta \phi$ with respect to horizontal betatron oscillations is as follows: from the center of one straight section to the center of the next, with a closed magnet between, $\Delta \phi=3.75^{\circ}$; for the corresponding distance with an open magnet between, $\Delta \phi=11.25^{\circ}$. The sum must, of course, be $15^{\circ}$ since the distance then involved constitutes $1 / 24$ of the entire magnet ring. Some theorists prefer to deal with $\left(\nu_{x}\right)(\Delta \phi)$; since $\nu_{\mathrm{x}}=6.4$, the angles $3.75^{\circ}$ and $11.25^{\circ}$ become $24^{\circ}$ and $72^{\circ}$. Further information is presented in CEA-90. Phase-angle lengths with respect to vertical oscillations are similar, but are in opposite relationship.

\subsection{Phase Stability}

Synchrotron oscillations are, of course, a testimonial to the phase stability inherent in the synchrotron. If the design of the system of accelerating chambers has bccn chosen properly and $\mathrm{rf}$ power of suitable frequency is provided, the orbiting electrons tend to remain in synchronism with the accelerating fields. That is, phase stability exists. To see why this is so, one may consider an electron that arrives at a given chamber a short time after the ideal moment. (At the ideal moment the longitudinal electric field is positive and is decreasing; see Chap. 10.) Because it arrives late, the electron receives a smaller-than-normal forward push, proceeds onward with a smaller-than-normal momentum, and is deflected by the ring of magnets into a smaller-than-normal orbit. Because its speed is practically constant (i.e., practically c), the electron travels around this orbit more quickly than normal. In short, it tends to catch up. Typically, it may travel around the orbit of the order of 1 to 20 times before catching up. And, of course, it then tends to overshoot. The reverse process then corrects the situation; the electron arrives at each cavity too early, encounters an abnormally large field, gains an abnormally large amount of momentum, hence travels in a larger orbit and takes more time to complete 
each turn. (The whole process is such that the designers of electron synchrotrons sometimes say that the electron has, in effect, negative mass: if the electron is pushed forward it takes longer to complete a turn than if it were pushed backwards.)

It is because of the phase stability of a synchrotron that a gradual increase in magnetic field leads to a corresponding increase in electron energy. To keep in step with the rf, the electron must follow an orbit of constant length - hence constant radius. But the increasing field of the magnets necessarily decreases the radius unless the electron gains momentum. Thanks tu phase stability, just the necessary gains of energy and momentum occur.

At injection, when the energy demand $\mathrm{dB} / \mathrm{dt}$ is very small (corresponding to about $0.2 \mathrm{Mev}$ per turn) and the energy loss by synchrotron radiation is negligible, the Operator may employ a peak potential of about $20 \mathrm{kv}$ across the individual $\mathrm{rf}$ cavity gap. The synchronous phase angle of the accelerator is then about $160^{\circ}$ and the (total) length, or phaseangle dimension, of the $\mathrm{rf}$ bucket is in the neighborhood of $300^{\circ}$. The length of the $\mathrm{rf}$ bucket depends on the width of the useful region of the magnet gap, and begins to decrease if the operator raises the cavity voltage above a certain value. At injection the height, or energy dimension, of the $\mathrm{rf}$ bucket is of the order of $3 \%$ of the electron energy; the value depends on the width of the useful region of the magnet gap.

Midway in the acceleration interval during 6-Bev operation, i.e., when the electron energy reaches $3 \mathrm{Bev}$, the energy demand $\mathrm{dB} / \mathrm{dt}$ is very large, corresponding to about $0.8 \mathrm{Mev} / \mathrm{turn}$, and synchrotron radiation losses are beginning to be appreciable. The Operator may employ cavity potentials of 50 to $100 \mathrm{kv}$, the synchronous phase angle of the accelerator is perhaps $150^{\circ}$ to $165^{\circ}$, the length of the $\mathrm{rf}$ bucket is of the order of $50^{\circ}$, and the height of the bucket is about $0.1 \%$ of the electron energy.

Near the end of the acceleration interval during 6-Bev operation, $\mathrm{dB} / \mathrm{dt}$ becomes negligible but the radiation losses are very large, namely about $4.5 \mathrm{Mev} / \mathrm{turn}$, as indicated in Chap. 10. If the operator is using the beam-bump method of target engagement (see Chap. 16), he desires to use a peak cavity voltage that is amply large enough to compensate for the radiation loss; usually he uses a voltage of about $200 \mathrm{kv}$. The synchronous phase angle is then about $130^{\circ}$ or $135^{\circ}$, the length of the $\mathrm{rf}$ bucket is about $70^{\circ}$, and the height is about $0.2 \%$. 


\section{Appendix to Chapter 3}

\section{Some Basic Definitions}

Synchrotron: An accelerator in which bunches of charged particles travel many times around an orbit (defined by a magnetic guide field) of approximately fixed radius. and, at certain stations along the orbit, are accelerated by synchronized longitudinal electric fields that increase the momentum of the particles in proportion to the increase in strength of the guide field.

Strong-focusing synchrotron: A synchrotron employing a clrcular array of magnets that provide not only a magnetic field of appropriate direction and magnitude but also an alternating series of field gradients that confines most of the orbiting particles to a region of very small cross-section.

Equilibrium orbit: A concept applicable to a particle of given energy and given momentum at a given instant during the magnet excitation cycle. Specifically, it is the sole orbit-path that lies well within the magnet gaps and closes perfectly on itself, i.e., the sole path that the given particle can retrace repeatedly as it makes successive turns around the ring. (The simplifying assumption is made here that the strength of the magnetic field does. not change appreciably during the time required for the particle to make the successive turns mentioned.) If the particle follows any other path, this latter does ...... not close on itself, but is necessarily "different every time" since the betatron frequencies are non-rational numbers. The equilibrium orbit is roughly, but not exactly, circular. It serves as a convenient reference line from which to measure the directions and amplitudes of betatron oscillations.

\section{Betatron oscillation: A transverse oscillation (of an orbiting particle) the frequency} of which depends on the focusing forces provided by the magnets. The oscillation is transverse to the direction of travel and is approximately symmetrical with respect to the equilibrium orbit appropriate to a particle. having the given momentum. The oscillation. is nearly independent of any forces due to the rf cavities and indeed can occur even when the $\mathrm{rf}$ power is turned off and the particle is coasting. It is sometimes called a free 
oscillation. The name betatron oscillation stems from the fact that oscillations of this type are prevalent in betatron-type accelerators. Betatron oscillations may be vertical or horizontal or in any other direction transverse to the main direction of travel. They are never longitudinal. The frequencies of the vertical and horizontal oscillations are called $\nu_{z}$ and $\nu_{\mathrm{x}}$, and are expressed in the unit cycles per turn, rather than cycles per second.

Synchrotron oscillation: A longitudinal oscillation (of an orbiting particle) the frequency of which depends on the forward-and-backward forces impressed on the particle as it passes through the $\mathrm{rf}$ cavities. If the $\mathrm{rf}$ power is turned off, the synchrotron oscillations cease. The frequency of such oscillation varies enormously as the acceleration continues. An increase in $\mathrm{rf}$ power level tends to increase the forces concerned and increase the frequency, but the increasing mass of the particle tends to decrease the frequency. The net effect is a decrease that may amount to a factor of 10 or more by the end of the acceleration interval.

Displacement component of momentum vector of the equilibrium orbit: If, at some given instant in the acceleration interval, two of the orbiting electrons have different momenta, they have equilibrium orbits of different radius. Let the difference in radius be called D. In the special case in which the momentum of the first electron is ideal and that of the second electron is twice the ideal, $D$ is called the "displacement component of momentum vector." The $\mathrm{D}$-value depends on whether the difference in radius is measured at the center of a closed magnet, center of an open magnet, or center of a straight section. Often a synchrotron designer prefers to deal with $\mathrm{D}_{\text {ave }}$, the value obtained by averaging $\mathrm{D}$ with respect to $\mathrm{s}$, the distance along the ideal orbit. Of course, a twice-normal momentum is highly unrealistic since the gradients of field are uniform throughout a narrow region only; but to define the displacement component of momentum in this way makes for computational convenience.

Momentum compaction factor $a_{\mathrm{p}}$ : . Ratio of (1) D value actually pertaining to the synchrotron to (2) D value that would be found if the strength of the guide field was everywhere equal to the value at the ideal orbit. An equivalent definition is $D / r_{i}$, where $r_{i}$ is : the radius of the ideal orbit. A synchrotron designer usually deals with the average value of momentum compaction, defined as $\frac{\mathrm{D}_{\text {ave }}}{\left(\mathrm{r}_{\mathrm{i}}\right)_{\text {ave }}}$. See also CEA-25. 
Damping of an oscillation: A gradual decrease of amplitude of oscillation. In the CEA, damping may occur as a result of (a) decrease of energy of oscillation, i.e., decrease due to radiation damping, or (b) increase of mass of the electron, i.e., adiabatic damping. The amplitude of vertical betatron oscillation of a freshly injected electron decreases steadily for both reasons. The amplitude of horizontal betatron oscillation tends to decrease for reason (b) but, when the electron energy is very high, the amplitude tends to increase because energy is fed into the oscillation by the anti-damping process explained below.

Anti-damping of horizontal betatron oscillation: A progressive increase in amplitude of horizontal betatron oscillation of an electron as a consequence of the greater probability of photon emission when the electron path is curving most sharply, i.e., when the elcctron is situated farthest outward from the equilibrium orbit. After the photon has been emitted, the electron has less momentum, the pertinent new equilibrium orbit is farther in, and consequently the electron finds itself oscillating with greater amplitude than before. The process tends to occur repeatedly, producing an ever-growing horizontal oscillation.

Orbit distortion: An irregular change in the shape of the equilibrium orbit as a result of irregularity in the pattern of magnetic fields through which the particle travels. Such an irregularity will occur if a magnet has been positioned improperly or is powered improperly, or if there are stray magnetic fields in the neighborhood.

Isomagnetic line: A certain reference line, conceptually fixed with respect to a given magnet that is one of a set of magnets of a synchrotron ring. Consider the set of 96 half-magnets used at CEA. The set includes units of cilosed type allu units of open typo. Each unit is a straight array of core blocks. Suppose that all the closed half-magnets are situated distance $D_{1}$ from the accelerator centerpoint and all the open half-magnets are situated distance $D_{2}$ from the centerpoint. It is possible to find a distance $D_{3}$ such that when, in the median plane of each half-magnet, a line $L$ is drawn at this distance $\mathrm{D}_{3}$ from the centerpoint, the magnetic fields at these lines will have the identical magnitude. The line $L$ in a given half-magnet is called the isomagnetic line of that half-magnet, with respect to the given arrangement of the magnets.

If now, all closed-type magnets are moved radially inward by a given amount and all open-type magnets are moved radially outward by this same amount, the isomagnetic 
lines may remain practically unchanged in position relative to the earth, but will be shifted with respect to the individual magnets.

The basic 96-sided polygon: The regular, 96-sided polygon defined by the 96 lines $\mathrm{L}$ defined above. Because the field strength is identical along all of these lines, the set of lines serves as a reference frame for describing the equilibrium orbit (assuming a given instant in the magnet excitation cycle and an electron of appropriate momentum). The equilibrium orbit itself is, of course, curved; but the 96-sided polygon comprising the 96 işmagnetic lines represents the best fit that can be achieved with a regular 96-sided polygon.

Acceptance ellipse of a synchrotron: A specification of all the acceptable pairs of values of angular deviation and transverse linear deviation of the freshly injected particle at the location where it enters the synchrotron, i.e., every pair that is consistent with capture of the particle into a stable orbit. If each such pair is represented by a single point on a graph the $x$ and $y$ coordinates of which correspond to angular deviation and linear deviation, the set of points occupies an elliptical area. If the ellipse is not tilted, one may describe the ellipse adequately by stating the lengths of the $x$ and $y$ semi-axes. In all, two ellipses are involved: one for deviations in the vertical plane, the other for deviations in the horizontal plane. See CEA-90.

Beta function with respect to horizontal betatron oscillations: The maximum horizontal displacement of an electron from the equilibrium orbit at a given location $s$ along the orbit as the electron travels around the orbit many times while executing a horizontal betatron oscillation of amplitude such that when the electron crosses the equilibrium orbit at the given location $s$ its path makes an angle of 1 radian with that orbit. Obviously, an angle-of-crossing of 1 radian is not realistic; but to define the beta function in terms of this unit angle makes for computational convenience. For example, in a practical case in which the actual angle-of-crossing of an electron at a specific location $s$ is 0.001 radian, the maximum displacement at $s$ is simply $(0.001) \beta(s)$. The beta function has its greatest value $\beta_{\max }$ at the center of a horizontally focusing (closed) magnet and its smallest value $\beta_{\min }$ at the center of a vertically focusing (open) magnet. [A somewhat different subject is the half-width $W$ of the band swept out by an electron that travels around the orbit many times and, when crossing the equilibrium orbit at the center of a closed magnet, makes an 
angle of 1 radian with that orbit. The half-width $\mathrm{W}$ at any location $\mathrm{s}$ is given by $\sqrt{\beta(s) \beta_{\max }}$. If the angle-of-crossing at the center of the closed magnet is 0.001 radian, the half-width $\mathrm{W}$ at any location $\mathrm{s}$ is $(0.001) \sqrt{\beta(\mathrm{s}) \beta_{\max }} \cdot \mathrm{A}$ corresponding beta function can be defined for vertical betatron oscillations; their maximum displacements occur at the midpoints of vertically focusing magnets.

Phase angle $\phi$ of an orbiting electron that is executing a horizontal betatron oscillation: A specially distorted measure of the electron's position around the ring, the distortion being specified with respect to the beta function $\beta(s)$ and the betatron oscillation frequency $\nu_{\mathrm{x}}$ thus: $\frac{\mathrm{d} \phi}{\mathrm{ds}}=\frac{1}{\nu_{\mathrm{x}} \sqrt{\beta(\mathrm{s})}}$. The definition is such that:

One complete trip around the ring corresponds to a $360^{\circ}$ increase in $\phi$, and $\mathrm{D}_{\mathrm{eo}} / \beta$ varies sinusoidally with $\left(\nu_{\mathrm{x}} \phi\right)$, where $\mathrm{D}_{\text {eo }}$ is the horizontal displacement of the electron from the equilibrium orbit.

By dealing with $\phi$, rather than $s$ and rather than the actual physical angle, theorists find that various computations of orbits, oscillations, etc., are much simplified. A corresponding. phase angle may be defined for electrons executing vertical betatron oscillations; however, it is conventional that the choice of location of zero angle differs by the center-tocenter distance between closed and open magnets.

Synchronous phase angle: During any given microsecond of the acceleration interval, the electric potential across a given gap of a given rf cavity varies approximately sinusoidally. If $\mathrm{V}_{\mathrm{p}}$ is the potential at the moment the forward-accelerating force on the electron is greatest, the instantaneous potential $V_{i}$ may be expressed as $v_{i}=V_{p} \sin \omega t$. $=\mathrm{V}_{\mathrm{p}} \sin \phi$, where $\phi$ is the "synchronous phase angle." Usually one assumes that $-180^{\circ} \leq \phi \leq 180^{\circ}$. If a given electron passes through the midpoint of the gap at some instant $t_{s}$, the quantity $\phi_{s}=\omega t_{s}$ is called the "synchronous phase angle of the electron." If this phase angle happens to be ideal, in the sense that the electron continues to follow an orbit of unchanged radius (i.e., it receives just enough energy to compensate for radiation losses and to satisfy the energy demand stemming from the increasing magnetic fields in the 48 magnets), it is called the "synchronous phase angle of the accelerator."

Phase-space specification of a particle in a beam: A six-dimensional specification comprising six kinds of deviations-from-ideal: linear and angular deviations in the vertical 
plane, linear and angular deviations in the horizontal plane, and also deviations of energy. and longitudinal position. Actually, longitudinal position is usually expressed in terms of synchronous phase angle of the electron.

RF bucket: A specification of all the acceptable pairs of values of synchronous phase angle and energy deviation (of an orbiting, accelerating particle at a given location along the orbit and assuming a given value of $\mathrm{B}, \mathrm{dB} / \mathrm{dt}$, and $\mathrm{rf}$ power level), i.e., every pair that is consistent with the particle's remaining in synchronism with the accelerating fields. If each pair is plotted as a single point on a graph the $x$ and $y$ coordinates of which correspond to synchronous phase angle and energy deviation, the set of points occuples an area that, under some circumstances, has some resemblance to an ellipse or to a longitudinal cross-section of a football. Since the electrons travel in a succession of bunches, a succession of such football-shaped areas is implied, and is reminiscent of the chains of buckets used in some types of materials-transport machines.

References to Strong Focusing Electron Synchrotrons in General

M. S. Tivingston and J. P. Blcwett "Particle acceleraturs,". McGiraw Hill (1962). (see also the many references listed at the end of the book.)

J. J. Livingood "Principles of Cyclic particle accelerators," Van Nostrand (1961).

R. R. Wilson "Electron synchrotrons," A chapter in Vol. 44 of the Encyclopedia of Physics, Springer, Berlin (1959).

G. K. Green "Theory of Alternating gradient accelerators," pp 300-318 of Vol. 44 of the Encyclopedia of Physics, Springer, Berlin (1959).

E. D. Courant and H. S. Snyder "Theory of alternating gradient synchrotrons," Annals of Physics $\underline{3}$, p. 1 (1958).

E. D. Courant, M. S. Livingston, and H. S. Snyder "The strong-focusing synchrotron: a new high energy accelerator," Phys. Rev. 88, 1190 (1952).

References to the Basic Plan of CEA

M. S. Livingston "Design study for an A.G. electron synchrotron," CAP-1 of 4/26/54.

M. S. Livingston "Proposal for a 6-Bev electron accelerator," CAP-2 of 12/15/55.

M. S. Livingston "Study of accelerator grade level," CAP-22 of 2/28/56.

M. S. Livingston "The Cambridge Electron Accelerator," CEA-1 of 5/4/56. 
M. S. Livingston "Present status of components and systems; outline," CEA-TM-41 of $11 / 17 / 58$.

M. Widgoff "Accelerator parameters," CEA-TM-50 of 9/4/59.

CEA Staff: "The Cambridge Electron Accelerator," CEA-81 of Aug. 1, 1960.

M. S. Livingston and W. A. Shurcliff "The Cambridge Electron Accelerator," Science 134, 1186 (1961).

Other Pertinent References

W. I. B. Smith "Injection path, phase space, and momentum matching," CEA-90 of 8/31/62. 


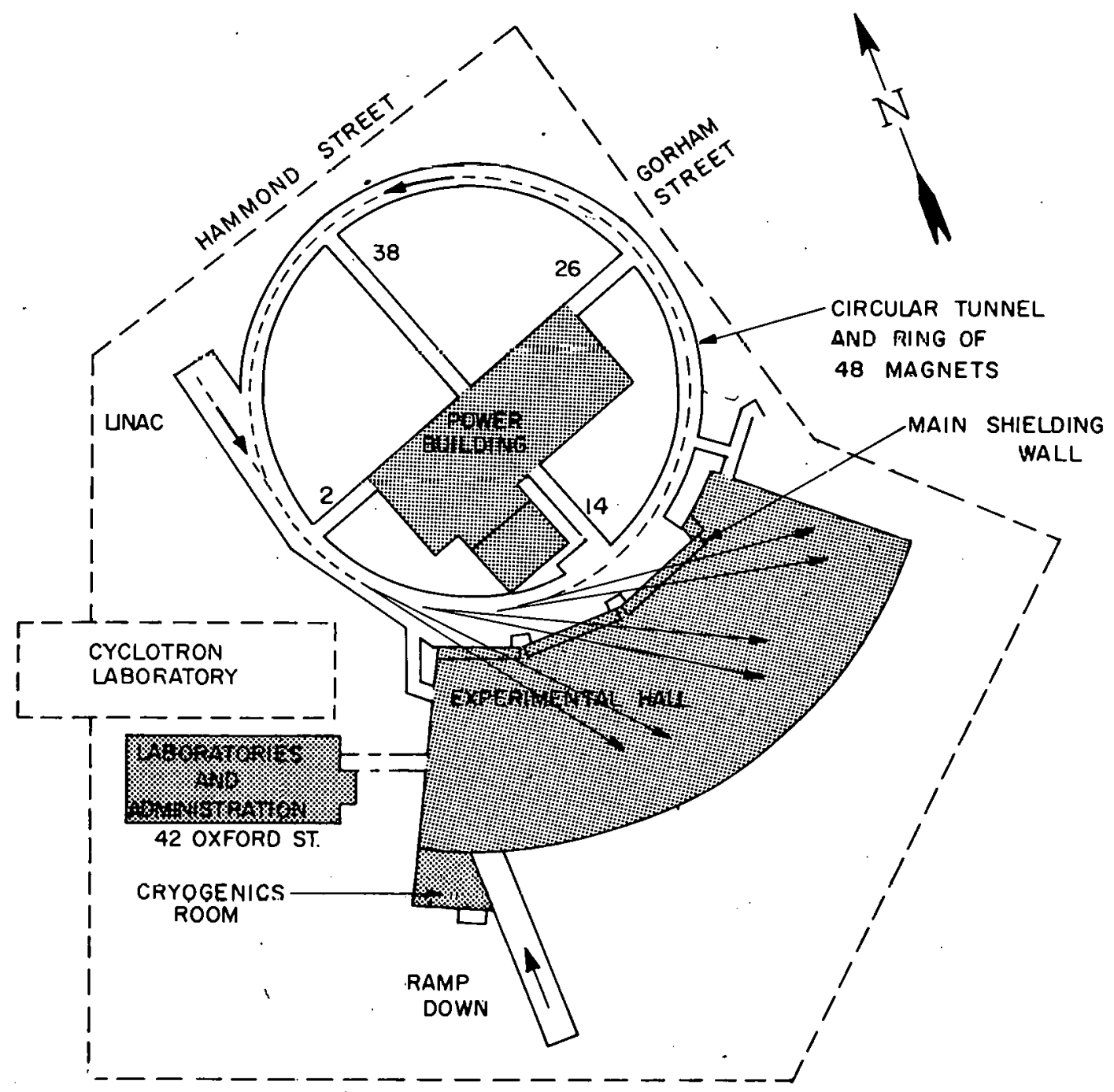

Fig. 3.1. Plan of Cambridge Electron Accelerator, located on Harvard University grounds adjacent to the Physics Department, the Division of Engineering and Applied Physics, and the Cyclotron Laboratory. Not drawn to scale. 


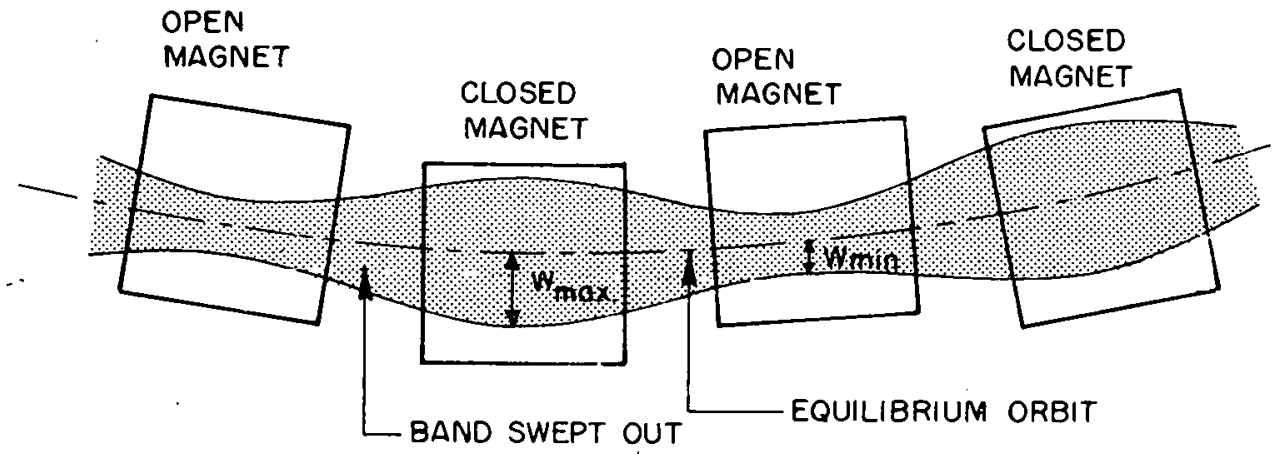

Fig. 3.2. Schematic diagram indicating the greater width, in closed magnet as compared to open magnet, of the band swept out by a single electron that executes a horizontal betatron oscillation while traveling around the orbit many times. The ratio $\mathrm{W}_{\max } / \mathrm{W}_{\min }$ of extreme half-widths is $0.60 / 0.24$.

\section{EQUILIBRIUM ORBIT}

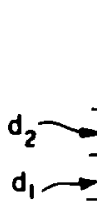

$\uparrow$ TO CENTERPOINT OF ACCELERATOR

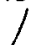

$63.9^{\prime \prime}$

Fig. 3.3. Schematic plan-view diagram of equilibrium orbit segment that lies in one half of a closed magnet. The equilibrium orbit moves outward if the frequency of $\mathrm{rf}$ power is decreased; that is, $d_{1}$ increases and $d_{2}$ decreases. But the sum $\left(d_{1}+d_{2}\right)$ remains almost constant, being about 0.47 inches. The radius of curvature of the arc shown is about $86 \mathrm{ft}$., and the mid-point of the arc is about $118 \mathrm{ft}$. from the centerpoint of the accelerator. 


\section{Chapter 4}

\section{The 48 Magnets}

\subsection{Purpose}

4.2 Principle of design

4.3 Design

4.4 Manufacture

4.5 Performance

\section{$4.1 \quad$ Purpose}

The ring of 48 magnets serves two major functions: it provides a verticallyupward-directed magnetic field that deflects the electrons to the left, so that they travel counterclockwise in an approximately circular orbit, and it provides a succession of oppositely directed magnetic gradients that collectively "focus" the electron paths both horizontally and vertically so that they remain near the center of the vacuum chamber.

\subsection{Principle of Design}

General Considerations: In order that electrons may be accelerated to an energy of 6 Rev while traveling in an approximatcly circular path aboul $118 \mathrm{fl}$. in radlus, the magnets defining the path must provide, at the end of the acceleration interval, a vertical field $\mathrm{B}_{\mathrm{z}}$ of about 7000 to 8000 gauss. Since a field of this magnitude is required throughout a considerable area (aggregating about $300 \mathrm{sq}$. ft.), the cost of power for excitation is large and accordingly the use of iron cores is indicated. Because a synchrotron repetition rate of 60 cycles/sec was adopted, the magnet cores were constructed of thin, insulated laminations oriented parallel to the field direction; this arrangement insures that the eddy currents induced in the blocks remain small enough so that eddy-current heating, and likewise any distortions of the field in the magnet gap, will be insignificant. The laminations. in any one magnet are of identical shape and have been bonded into simple straight stacks, or core blocks; but because the orbit is curved, the blocks have been arranged in two straight groups which make a small angle with one another and thus conform roughly to the circular orbit. The blocks of an individual group are held in accurate alignment by means of guide rails resting on a sturdy girder; the blocks are held a sufficiently great distance above the girder that practically no magnetic flux is induced in the girder and therefore no distortion of the field in the magnet gap is produced. 
The magnets are excited by coils supplied with full-biased a.c., rather than pure a.c., for reasons indicated in Chap. 6.

The magnet jaw, or gap, is large enough to permit inserting the excitation coils, to permit installing the vacuum chamber, and to allow room for pole face windings.

Shape of the Pole Face: In order that strong focusing may be achieved, two types of pole-face shape, called closed and open, are used, and the resulting closed and open magnets are arranged alternately around the ring. As indicated in Fig. 4.1, the gap in the closed magnet is smallest at the front (outer) portion of the pole tips, i.e., the portion at the greatest distance from the accelerator centerpoint. The reverse is true of the open magnet.

An essential feature of the closed magnet is that, within the gap, the quantity $\frac{\partial \mathrm{B}}{\partial \mathrm{x}}$, called the radial gradient of vertical component of magnetic fteld, is large and positive. That is, $B_{z}$ increases rapidly with $\mathrm{x}$; hence the magnet is called radially focusing. [It follows that the conjugate quantity $\frac{\partial \mathrm{B}}{\partial z}$, the vertical gradient of the horizontal component of field, is also large and positive. This follows from Maxwell's equations, which state that, in a region free of time-varying electric fields, $\left(\frac{\partial B_{z}}{\partial x}-\frac{\partial B}{\partial z}\right)=0$. Thus at any given point in the gap, the two gradients are alike in sign and magnitude.]

In the gap of an open magnet the gradient $\frac{\partial B_{z}}{\partial x}$ is negative; $B_{z}$ decreases rapidly with increasing distance from the accelerator centerpoint, and the magnet is called radlally defocusing. Again $\left(\frac{\partial \mathrm{B}_{\mathrm{z}}}{\partial \mathrm{x}}-\frac{\partial \mathrm{B}}{\partial \mathrm{z}}\right)=0$.

The exact shapes of the pole faces were chosen su as to satisfy the following more specific requirements on the gradient $\frac{\partial \mathrm{B}}{\partial \mathrm{x}}$ :

Requirement \#1: That the magnitude of the gradient at the isomagnetic line have a large and accurately controlled value appropriate to producing satisfactory values (discussed below) of the horizontal and vertical betatron frequencies $\nu_{\mathrm{x}}$ and $\nu_{\mathrm{z}}$.

Requirement \#2: That the magnitude of the gradient vary slightly and in accurately controlled manner with the radial position $x$, such that the betatron frequencies will remain almost unchanged if the electron follows an orbit having a radius. slightly greater than, or slightly less than, the ideal radius.

In connection with Requirement \#1, the reader will recall that a central goal of the designer of an electron synchrotron is to accommodate, at any given moment, electrons having an appreciable spread of momentum. The freshly injected electrons may have 
a spread as large as 1 or $2 \%$, and if they are to be accommodated in a vacuum chamber only a few inches wide a high degree of momentum compaction is required. This in turn requires that $\frac{\partial B_{z}}{\partial x}$ be large.

In evaluating the momentum compaction, an accelerator designer usually deals with the momentum compaction factor, defined as the ratio of actual radial spread of the equilibrium orbits of electrons having different momenta to the spread that would result if the magnetic field strength did not vary with radial position. (This is discussed in CEA-25.) Since the value of compaction factor depends on whether the radial spread is measured in a closed magnet or an open magnet, the designer usually works with the average momentum compaction factor, called $a_{p}$. It may be shown that $a_{p}$ varies approximately as $(1 / \nu)^{2}$, and that a $\nu$-value of 6.4 leads to an $a_{p}$-value of 0.031 ; these are the values that apply to the CEA magnet ring.

How closely must the $\nu$-value be controlled? If the nominal value is 6.4 , a variation as large as 0.05 , for example, does little or no harm. Yet if at any time during the acceleration interval the value decreases enough to reach the value 6.0 , beam blow-up will occur very quickly; the value 6.0 is called an integral stop-band, meaning that an electron executing just 6.0 oscillations per turn in a slightly irregular magnet ring will experience the same transverse "kick" each turn around the orbit and hence will oscillate with everincreasing amplitude and will soon be lost from the beam. A value of 6.5 , called a halfintegral stop-band, also leads to beam blow-up, although less rapidly. Accordingly the . CEA designers attempted to keep the betatron frequencies within these limits and (in order to reduce orbit distortions) nearer to 6.5 than to 6.0. Hence the choice of 6.4.

There would be some benefit from employing pole-face profiles producing much higher betatron frequencies. But the disadvantage would be serious: the pole faces would have to slope more steeply, causing difficulty in maintaining uniformity of gradient over a region of adequate width (see Requirement \#2) and difficulty in conforming to the necessarily closer tolerances needed to avoid stop-bands.

In the theory of alternating gradient synchrotrons that - unlike the CEA - employ magnets that are curved to coincide with the curved orbit of radius $r$ and are separated by straight sections of negligible length, an outstandingly important parameter is the $\mathrm{n}$ value, defined as $\frac{\Delta \mathrm{B}_{\mathrm{z}} / \mathrm{B}_{\mathrm{z}_{\mathrm{O}}}}{\Delta \mathrm{r} / \mathrm{r}_{\mathrm{O}}}$, where the subscript "o" means "at the equilibrium orbit." The parameter may be thought of as being the slope of a curve of "normalized $B_{Z}{ }^{n}$ vs. 
"normalized r." (The parameter is discussed in CEA-29, for example.) Although such theory does not apply rigorously to the CEA (because each straight section is long and each half-magnet is straight), it may be of interest that a $\nu$-value of 6.4 corresponds roughly to an $\mathrm{n}$-value of 91 . This means, for example, that two electrons that follow orbits differing in radius by $0.01 \%$ will find themselves in fields whose intensities differ by an amount of the order of $0.91 \%$.

For convenience in design and manufacture of the CEA magnet ring, the magnitude of the gradient $\frac{\partial \mathrm{B}}{\partial \mathrm{x}}$ at the isomagnetic line is made almost the same in the two types of magnets. A slight difference is required because the two kinds of magnets have slightly different end-effects, discussed in a following section.

In connection with Requirement \#2 the reader will recall that an electron that has abnormally great momentum at some instant will execute synchrotron oscillations and hence will follow a path that is sometimes farther from the accelerator centerpoint and sometimes nearer to it, as compared with the path of an electron having typical momentum. Consequently if the frequency $\nu_{x}$ of the horizontal betatron oscillation of such an electron were to vary with orbit radius, coupling between this oscillation and the synchrotron oscillation would occur and the betatron oscillation might grow in amplitude and cause the electron to be lost to the walls of the vacuum chamber.

To avoid such coupling, the synchrotron designers strove to find a pole-face profile that would produce a spatial distribution of field gradient such that the betatron frequencies would remain constant even for electrons traveling as much as, say, one inch either side of the isomagnetic line. An additional reason for wishing the frequencies to be constant. over an appreciable radial range is the fact that, at injection, the radius of the equilibrium orbit is 0.3 inch less than at the end of the acceleration interval (or about 0.6 or 0.7 inch less if the frequency of the $\mathrm{rf}$ system is frequency modulated as indicated in Chap. 10).

If the pole-face profile were to correspond exactly to a hyperbola, the field gradient at some typical location along the magnet would be independent of radial position; but the frequencies of betatron oscillations would not be independent of radial position - because of the differences in electron momentum concerned (and also because of the fringing fields at the ends of the magnets). For this reason the designers adopted a slightly different profile, namely one that makes the betatron frequencies independent of radial positions and, incidentally, compensates for the fact that, at best, the poles could correspond only to short ( $\sim \frac{1}{2}$ inch) segments of the pertinent hyperbola. See Fig. 4.2. 
The hyperbola to which the finally-adopted shape is an approximation is usually specified in terms of the characteristic length $\mathrm{k}$, defined as the distance from (a) the vertical plane containing the isomagnetic line to (b) the vertical asymptote of the hyperbola. As indicated in Fig. 4.1, the vertical asymptote of the hyperbola pertinent to a pole of a closed magnet is situated deep within the magnet, and the curvature of such a pole is greater near the rear (inside) of the magnet jaw than near the front (outside). For the open magnet, the situation is the reverse: the vertical asymptote lies well in front of the magnet and the curvature of the pole is greater near the front. Knowing the characteristic length $\mathrm{k}$, one may compute $\left(\frac{\partial \mathrm{B}_{\mathrm{z}}}{\partial \mathrm{x}}\right)_{\mathrm{o}}$ from the relation $\left(\frac{\partial \mathrm{B}}{\partial \mathrm{x}}\right)_{\mathrm{o}}=\frac{\mathrm{B}_{\mathrm{z}_{0}}}{\mathrm{k}}$.

In the case of a synchrotron to which the concept of $n$-value is applicable, the $n$ value may be calculated from the relation $n=r_{0} / k$ where $r_{0}$ is the radius of curvature of the equilibrium path in the magnet.

Length of Magnet: The designers of the synchrotron chose a magnet length such that one period of the magnet ring (i.e., one closed magnet, one open magnet, and two straight sections) would be about one quarter of the wavelength of a betatron oscillation. If the length of the period were to greatly exceed one quarter of a wavelength, the amplitudes of betatron oscillations and the maximum value of the beta function would be unnecessarily large; if the length of a period were to be much less than one quarter of a wavelength, the number of magnets required would be undesirably large, a greater magnitude of field gradient would be needed, and the length of the individual straight section would be undesirably small.

Earlier decisions as to choice of diameter of the magnet ring, of field gradient, and of maximum electron energy had implied that the betatron oscillation wavelength would be of the order of $120 \mathrm{ft}$., and accordingly the designers adopted a value of about $30 \mathrm{ft}$. for the length of one period of the magnet ring. This was an especially satisfactory figure since it left the designers free to fix the total number of magnets at 48 ; being evenly divisible by $2,3,4,6,8,12,16$, and 24 , this number allows many choices of uniform spacings of radial tunnels, rf cavities, pick-up-coil tanks, etc.

The value adopted for the length of the individual magnet was about $12 \mathrm{ft}$. This implied that the length of the straight section between magnets would be about $3 \mathrm{ft}$., measured between the overhanging coils of the magnets and about $4 \mathrm{ft}$. measured between the terminal core-blocks. This length of straight section is sufficient to permit insertion of rf cavities and pick-up-coil tanks and to permit the ejection of near-tangential beams of 
photons and electrons. Adoption of a longer straight section would have necessitated shortening the magnets and increasing their field strength, which in turn would have increased the energy losses due to synchrotron radiation and would have increased the maximum value of beta function.

Sequencc of Magnets: During the early stage of designing the CEA, much attention was given to finding the optimum sequence of the two types of magnets. For a while, a sequence called "FOFDOD" seemed attractive, for reasons indicated in CEA-TM-24. ("F" stands for a radially focusing magnet, i.e., a closed magnet, " $D$ " stands for a radially defocusing magnet, i.e., an open magnet. "O" stands for an open space between magnets, i.e., a straight section.) In the FOFDOD sequence, a given open space is flanked by two magnets of identical type. To construct such a sequence, one would presumably mount an "F" core-block group along one half of a support girder and a "D" core-block group along the other half. The next girder would contain two such groups in the opposite sequence. A space of several feet would be left between girders to provide the open space desired, as indicated in Fig. 4.3.

The sequence finally chosen was a "FODO" arrangement, in which each open space is flanked by dissimilar magnets. Although this sequence leads to a slightly larger value of $\beta_{\max }$ (assuming a given value of $\nu$ ), it affords important advantages. Each girder carries core blocks of only one type; thus the construction of the magnet-plus-girder assembly is simpler, the construction of the vacuun eliamber is made simpler (since the cham ber must in any event have a different height in the two kinds of magnets), and the task of inserting the chamber is simpler. More important, since the different types of magnets rest on different girders, the two types can be moved radially inward or outward independently and oppositely - a process essential to any change in the betatron frequencies of electrons that have accelerated to high energy. To increase the betatron frequencies, one moves the closed magnets outward and the open magnets inward; such a move increases the distance between equilibrium orbit and the pole faces, hence decreases the field but leaves the gradient almost unchanged. (Pole face windings of small-gauge wire can be used to change the frequencies when the field strength in the magnets is low, i.e., at injection, but their effect is negligible when the field strength is high.)

The FODO arrangement has the additional advantage of providing a higher value of betatron frequency, i.e., about 6.4 as compared to perhaps 5.4, assuming use of the same magnitude of field gradient. Also, the momentum compaction factor provided by the FODO scheme is smaller. 


\subsection{Design}

The individual magnet has a C-shaped cross-section and consists essentially of stacks of thousands of C-shaped laminations. Each lamination is $0.014^{\mathrm{n}}$ thick and consists of Armco Grade A6 Trancor transformer steel, which is notable for its low coercive force and its high permeability at high field, as indicated in CEA-TM-13 and CEA-23. (The following section gives additional details as to the steel and the method of manufacturing the core blocks.) Each lamination has an electrically insulating coating.

Fig. 4.4 indicates the major dimensions of the individual lamination. The poles are 6.500" wide, and the central window is 9.500" high and 7.500" wide. At the bottom of the lamination there are two 2.500"-wide, 0.500"-high steps used in aligning the laminations during assembly into core blocks. Similar steps are provided at the top, to maintain symmetry.

The exact dimensions of the pole faces of closed-type and open-type laminations are indicated in Dwgs. D-11M-113-C and D-11M-191-A. The minimum distance between pole faces of the closed lamination is 1.560" and the corresponding dimension of the open lamination is 1.580". The gap height of the closed lamination is exactly $2.000^{\prime \prime}$ at a position $3 \frac{1}{2}$ " from the "front edge" of the poles, i.e., from the closed-gap side; the gap height

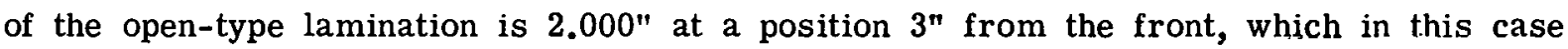
is the open-gap side. The closed edge of the pole face is rounded (with a $3 / 8^{\prime \prime}$ radius) to minimize undesirable edge effects there. A 5/8-inch-wide flat region is provided near the open edge to serve as a "shim" to widen the region in which the betatron frequencies are constant. See Fig. 4.2.

The design of the magnet and magnet ring is such that, at the instant of peak excitation during 6-Bev operation, the peak value of field strength $B_{z}$ at the isomagnetic line of either type of magnet (and at a location unaffected by end-effects) must be about 7600 gauss. It is estimated that, under such circumstances, the field strength in the back leg of the magnet is about 12,000 or 13,000 gauss, and that some degree of saturation may exist.

The pole-face profile is approximately hyperbolic and the characteristic length $k$ is 11.38". Accordingly the magnitude of the gradient in the neighborhood of the isomagnetic line (and at a location unaffected by end-effects) is approximately $\left(\mathrm{B}_{\mathrm{z}_{\mathrm{O}}}\right) / 11.38$ inches; that is, the field changes by about the reciprocal of 11.38 , or about $9 \%$, per inch of radial travel. The exact shape of the gently curving portion of the pole-face profile deviates 
from the hyperbola by an amount that nowhere exceeds 0.01 inch; the deviations are of such magnitude as to make the betatron frequencies independent of radial position throughout a range of \pm 1 inch. (If no deviations existed, the betatron frequencies would vary about $2 \%$ for a one-inch change in radial position.) The deviations of shape provide a rate-ofchange of gradient of $2.7 \%$ per inch in a closed magnet and $1.9 \%$ per inch in an open magnet.

The typical core block weighs $1027 \mathrm{lb}$, and the $\mathrm{rms}$ deviation is about $3 \mathrm{lb}$. The thickness is approximately 10.65". Further details may be found in "Specification of Magnet Core Blocks" dated $3 / 4 / 58$ and in a revision of $6 / 13 / 58$.

Each magnet includes 12 core blocks, arranged in two groups of six: At each end of such a group there is a stainless steel plate, and attached to each plate there is a packet of transformer-steel laminations that scrve to terminate the train of core blocks. Because the field here is a fringing field and is not perfectly vertical, but has a component that is parallel to the long axis of the magnet, the laminations are arranged in a vertical plane parallel to (rather than perpendicular to) this axis; see CEA-49. Although this terminal arrangement reduces the large eddy currents that would occur here, some eddy-current heating remains and accordingly water-cooling is provided. The set of six core blocks and two end-plates is maintained under longitudinal compression by ten stainless steel tie-rods. The length of the set of six core blocks is $63.9^{n}$, or $65.4^{n}$ if the two 3/4-inch-thick terminal packets are included.

Each group of six core blocks rests on front and rear guide rails which are pinned and bolted to the top of the supporting girder. Each block is pushed forward against the front rail to insure uniform radial alignment within about $0.002 "$. The upstream and downstream guide rails differ in orientation by $3.750^{\circ}$ and thus conform to the pertinent sides of the basic 96 -sided polygon. The guide rails are $1 \frac{11}{16}$ " high; consequently the air space between bottom of magnet and top of girder is sufficient so that very little field is induced in the girder and the field in the magnet remains symmetric about the median plane.

The girder, of box-type, is $144^{\prime \prime}$ long, $24^{\prime \prime}$ wide, and $13 \frac{3}{8} "$ high. When carrying no load, it is flat on top to within 0.002"; but when loaded with the core blocks and coils (and supported at the ends only, as explained in Chap. 5) it sags $0.015^{\prime \prime}$ at the front and $0.008^{\prime \prime}$ at the rear. It is believed that this "vertical scalloping" causes no appreciable distortion of the orbit. The girder is electrically grounded by a heavy copper conductor. The ends of the girder serve to support the adjacent rf cavities and straight section tanks and, indirectly, the high-vacuum pumps. 
Between the two groups of core blocks there is a wedge-shaped space, or junction, about 3 to $5 "$ long. It is used to admit the leads of the excitation coils and the coolingwater tubes.

Each magnet is energized by a 40-turn winding in the form of four flat horizontal coils. These are symmetrically located, two being above the median plane and two below. Between adjacent coils there is a sheet of 1/64" neoprene, to minimize chafing. Each coil encloses all 12 core blocks. Each is thin enough (about 1.5") to be inserted between the pole tips. The four coils are connected in series and together have a d.c. series resistance of $0.030 \mathrm{ohm}$ and an inductance of 33.2 millihenries. (An "effective 60-cycle a.c. resistance" can be defined, as in CEA-TM-86; the value is $0.125 \mathrm{ohm}$. )

The conductor used in the coils consists of a double layer of stranded cable. The individual strands ( 91 per cable) are insulated with Formvar to prevent eddy currents, and the pair of cables is embedded in a resin binder protected by a wrapping of fiberglass ribbon impregnated with epoxy resin. The combined cross-section of copper in each turn (i.e., in each pair of 91-strand cables) is about 0.39 square inch. The coils overhang the magnet at bath ends and make the overall length of the assembly about $13 \mathrm{ft}$; thus the net space between magnets is about $3 \mathrm{ft}$. The coils are cooled by water circulating in copper tubes meshed between the conductors. The coils are supported by stainless steel brackets at the ends and ccnter; throughuul must of their length they are left free, so that unavoidable vibration, amounting to a few mils, can occur without mechanical impact or friction. Further information is contained in "Specifications for Magnet Excitation Coils" of $2 / 3 / 58$, and a revision of $3 / 27 / 58$.

It was originally planned that magnets of open type would be situated 0.500 " farther inward radially than the closed magnets, so that the 2.000 -inch-high regions of the two kinds of gaps would be equidistant from the accelerator centerpoint. Later, as explained in the following chapter, the difference was reduced to $0.410^{\prime \prime}$. Fig. 4.5 explains the situation. See also Fig. 4.6.

\subsection{Manufacture}

The core blocks and end-plate assemblies were made by the Eddystone Division of the Baldwin Lima, Hamilton Corporation in 1959. The fabrication of the 450,000 laminations of Armco hot-rolled and annealed A6. transformer steel is described in detail in D. D. Jacobus' memorandum "Accelerator Magnet Fabrication" of $11 / 12 / 63$. The steel is said to 
contain 2.5 to $4.0 \%$ silicon, and at a field strength of 100 gauss it has a permeability exceeding 2000. The residual coercive force of specimens that have been subjected to a 10 oersted magnetizing field is said to be less than 0.3 oersted.

In collating the laminations into stacks (later to be bonded into core blocks), the manufacturer used a systematic "dealing" procedure that insured equal representation from each of the various melts of steel concerned. A shuffling procedure had been considered, but appeared more difficult and less satisfactory than dealing.

The laminations were bonded with Rubber and Asbestos Corporation's Resin \#AW119-E-20, which has properties somewhat similar to Goodyear Rubber Co.'s rubbermodified phenolic resin Pliobond \#20. Specially developed procedures for assembling the laminations in stacks, compressing the stacks, and curing them in an oven at $350^{\circ} \mathrm{F}$ were followed. The procedures insured accurate alignment of the laminations within 0.002 ", elimination of any excess of bonding material, and a $10.65^{n}$ overall thickness of the finished core block. Because the core blocks tend to cold-flow and become distorted if supported non-uniformly, they were always stored on flat, parallel, horizontal guide rails even during shipment.

The girders were made by Bethlehem Steel Co. Before the girders were painted, their upper surfaces were planed flat to within about $0.001 "$.

The coils were made by National Electric Coil Co.

The magnets were assembled by CEA early in 1960 and were mounted in the circular tunnel by Nov. 1960. In November and' December of that year some magnets were partially disassembled and then reassembled using different end-plates, to minimize performance differences attributed to variations in the magnetic properties of the end-plates. Also, an improved sequence of the magnets was arrived at, on the basis of magnetic-field tests and computer programs. By $2 / 1 / 61$ all the magnets were in place in the ring and, as indicated in Chap. 5 , the first radial survey had been begun.

\subsection{Performance}

To date, the core blocks and coils of the 48 magnets have performed excellently. No deterioration has been observed.

The effective length of the magnets, measured along the isomagnetic line, exceed the mechanical lengths because of the fringing fields that occur at the ends and produce "end extensions." Measurements reported in CEA-19, CEA-23, CEA-26, CEA-41, CEA-83, 
and CEA-TM-15 show that the end-extension-of-field of a closed magnet excited for 6-Bev. operation amounts to $0.9^{\prime \prime}$ and $0.8^{\prime \prime}$ at the outer end of a half-magnet and at the inner (junction) end respectively, and each of the two end-extensions-of-gradient amount to about $0.1 "$. The extensions of field of an open magnet are $0.9^{\prime \prime}$ and $0.8^{\prime \prime}$ and the extensions of gradient are $0.1^{n}$ and $0.0^{\prime \prime}$ at the outer and inner ends respectively. When the magnet excitation is low, e.g., at injection, the extensions are larger. The contour lines of end extension vs. radial position are oblique to the isomagnetic line rather than perpendicular to it, and the obliquity is a function of the excitation; also it is different for the two types of magnets. The obliquities pertinent to the outer end of a half-magnet differ considerably from those pertinent to the inner end. For further details see CEA-TM-15 and CEA-83.

The betatron frequencies applicable at peak magnet excitations appropriate to 2 or $3 \mathrm{Bev}$ operation have been measured and found to be 6.4. The same value is presumed to apply to 4, 5, or 6-Bev excitation. Approximately the same value applies (for a given excitation) throughout a \pm 1 -inch range in orbit radius. These findings, supported by actual measurements of field gradients, suggest that the gradients conform closely to the design values specified in a preceding section.

At injection, during 2-Bev operation of the synchrotron, the frequency $\nu_{x}$ of horizontal betatron oscillation, has been found to exceed 6.5 unless the pole-face quadrupole windings, discussed in Chap. 7, are brought into play. When the magnets are powered for 6-Bev operation the value of $\nu_{\mathrm{x}}$ at injection remains slightly below 6.5 even when the pole-face windings are not used.

The shape of the equilibrium orbit of a $6-\mathrm{Bev}$ electron is indicated in Chap. 3.

\section{$\underline{\text { References }}$}

More than sixty reports of the CAP, CEA, and CEA-TM series deal with the design, construction, and testing of the 48 magnets. Some of the more interesting of these are listed below.

\section{CEA Reports}

CEA-12 Orbit computation, effect of misalignment and twists on the equilibrium orbit. G. Lanza. 9/21/56.

CEA-19 Program for magnetic measurement. M. S. Livingston. $1 / 7 / 57$. 
CEA-23 High-field and flux measurement on the hybrid Mark II magnet. J. Dekleva and J. Rees. 2/27/57.

CEA-25 Motion of particles with momentum $p_{0}+\Delta p$ in an alternating gradient synchrotron. G. Lanza. 3/13/57.

CEA-26 On the existence of a magnetic and focusing length in a synchrotron. J. Dekleva and G. Lanza. 4/1/57.

CEA-29 Dynamic n-measurement on the CEA magnet model Mark II under a.c. and biased operation. H. Nysater. 4/16/57.

CEA-32 Amplitude and phase functions for the revised parameters of the CEA: G. Lanza and K. Steffen. 5/27/57.

CEA-36 Magnet construction and assembly. M. S. Livingston. 6/25/57.

CEA-37 Betatron oscillation frequency shift due to "n-errors." G. Lanza and K. G. Steffen. $7 / 1 / 57$.

CEA-41 Dynamic $B$ and n-behavior up to 7600 gauss in magnet model Mark II: comparison of results with long and small grad coils. V. P. Henri and H. Nysater. $8 / 13 / 57$.

CEA-45 Betatron oscillations and equilibrium orbits in terms of amplitude and phase functions. K. G. Steffen. 1/7/58.

CEA-49 End packets for reducing eddy current at ends of magnet cores. L. Young. $3 / 17 / 58$.

CEA-50 Relative oscillation amplitudes and effect of $\mathrm{rf}$ cavities on betatron oscillations in the new CEA structure. T. L. Collins and K. G. Steffen. 3/19/58.

CEA-51 Dynamic measurements with biased peaking strips of magnetic field and field gradient at low fields. H. Nysater. $3 / 28 / 58$.

CEA-62 Correction of magnetic field for momentum variation. K. W. Robinson. 9/2/58.

CEA-83 Magnetic measurements on the 48 synchrotron magnets. J. dePagter and H. Winick. 12/15/60.

\section{CEA-TM Reports}

CEA-TM-13 Choice of magnet steel. M. S. Livingston. 6/14/57.

CEA-TM-15 Aspects of fringing field effects on the focusing length of magnet sectors: J. Dekleva and H. Nysater. 7/5/57.

CEA-TM-16 Prototype pole face shape. M. S. Livingston. 7/11/57.

Other Reports

D. D. Jacobus "Accelerator Magnet Fabrication," 4 pp., 11/12/63. 

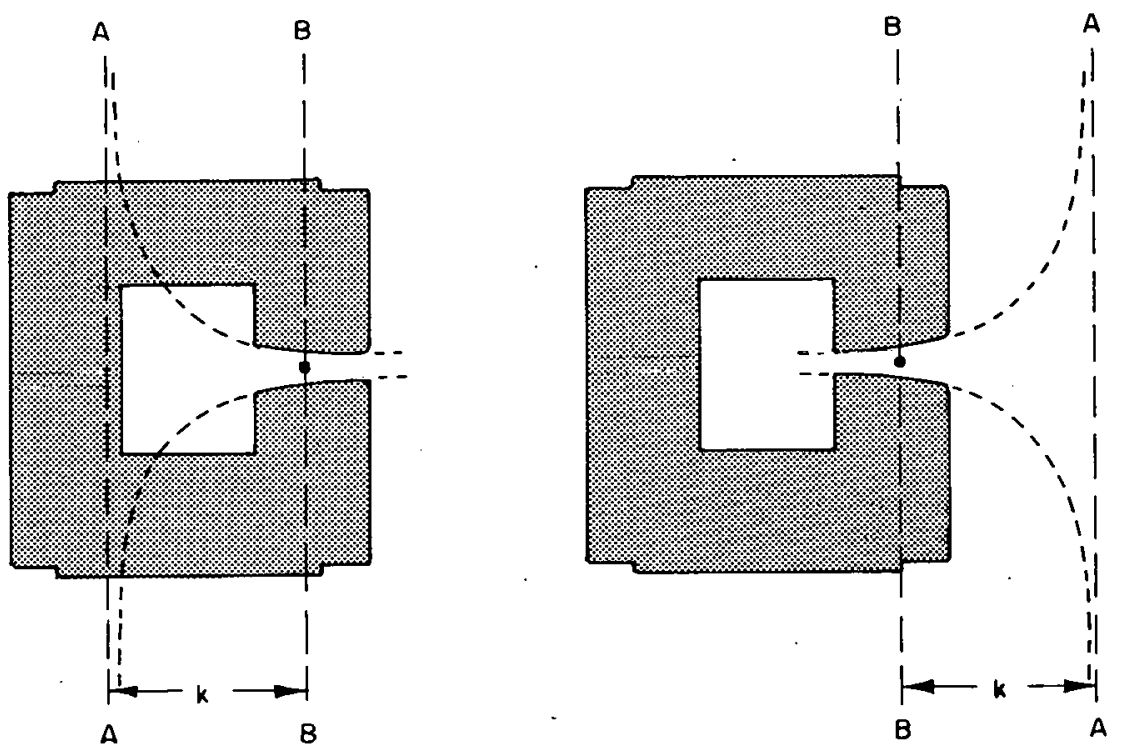

Fig. 4.1. Profiles of the poles of a magnet (schematic). The profile of each pole face conforms fairly closely to a portion of a hyperbola the vertical asymptote of which is indicated by line AA. The isomagnetic line, here seen "end-on," lies in the vertical plane $B B$. The distance $k$ is the characteristic length; its value is 11.38 inches.

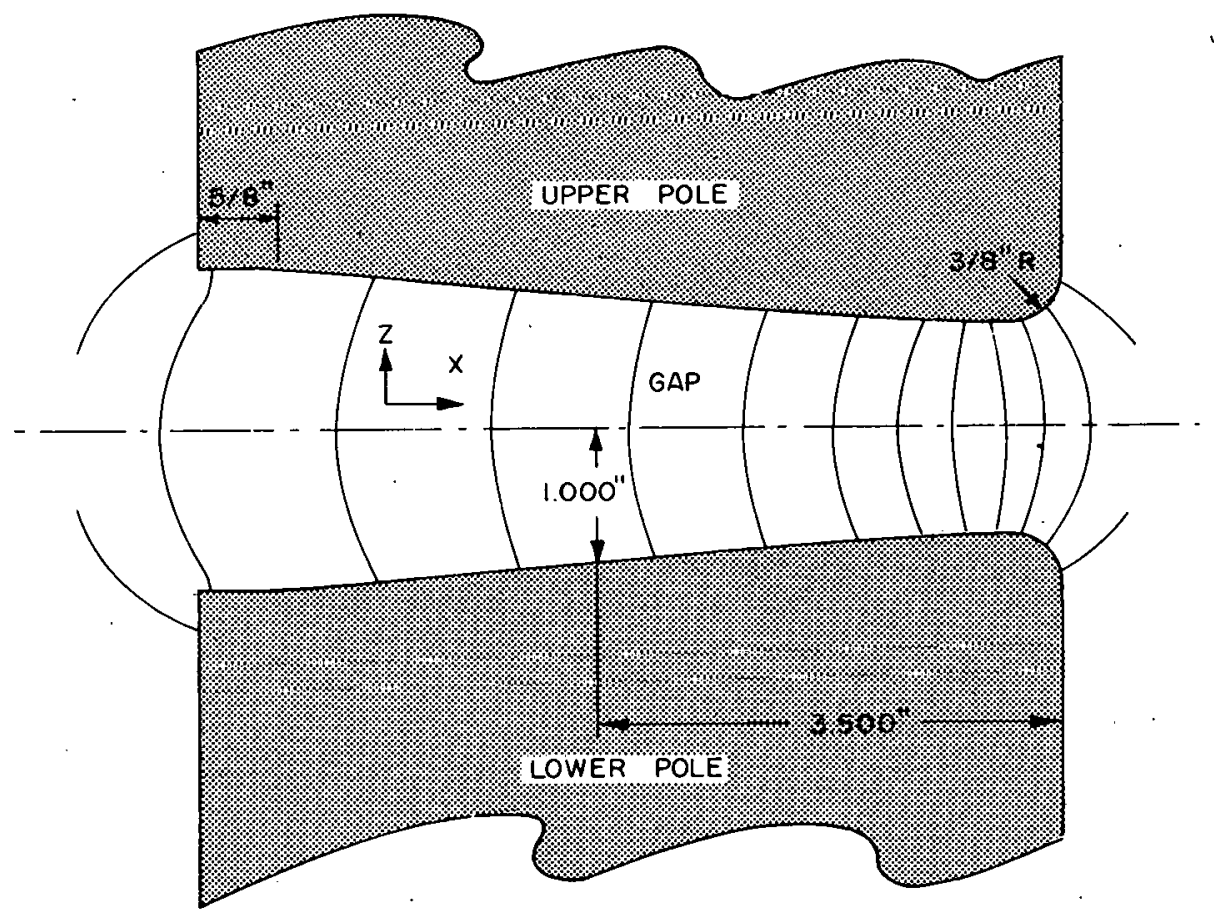

Fig. 4.2. Profiles of the poles of a closed magnet. Not to scale. The horizontal line indicates the mechanical median plane. Also included are lines that suggest (schematically only) the density and shape of the magnetic field lines. 


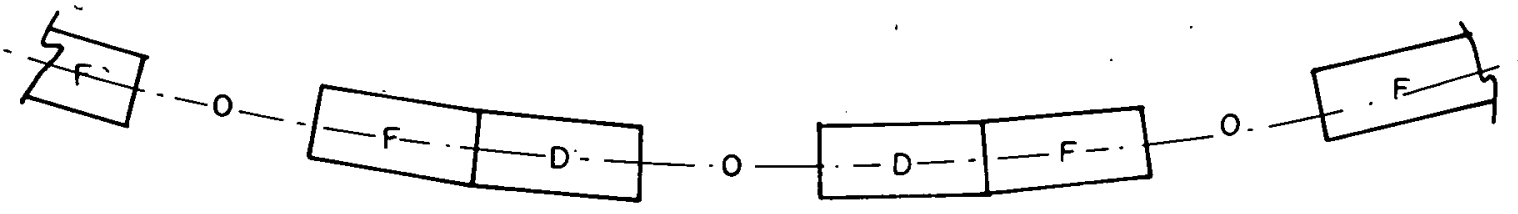

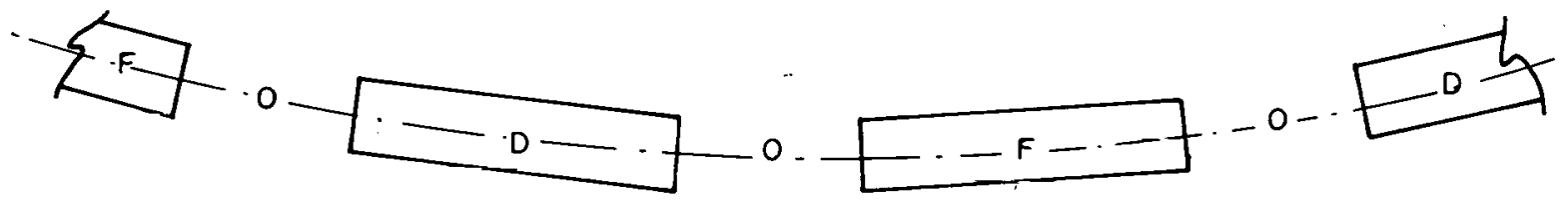

Fig. 4.3. Comparison of two sequences of radially focusing $(F)$ and radially defocusing (D) magnets in a strong-focusing synchrotron. The upper sequence is called FOFDOD. The lower sequence, FODO, is employed in the CEA.

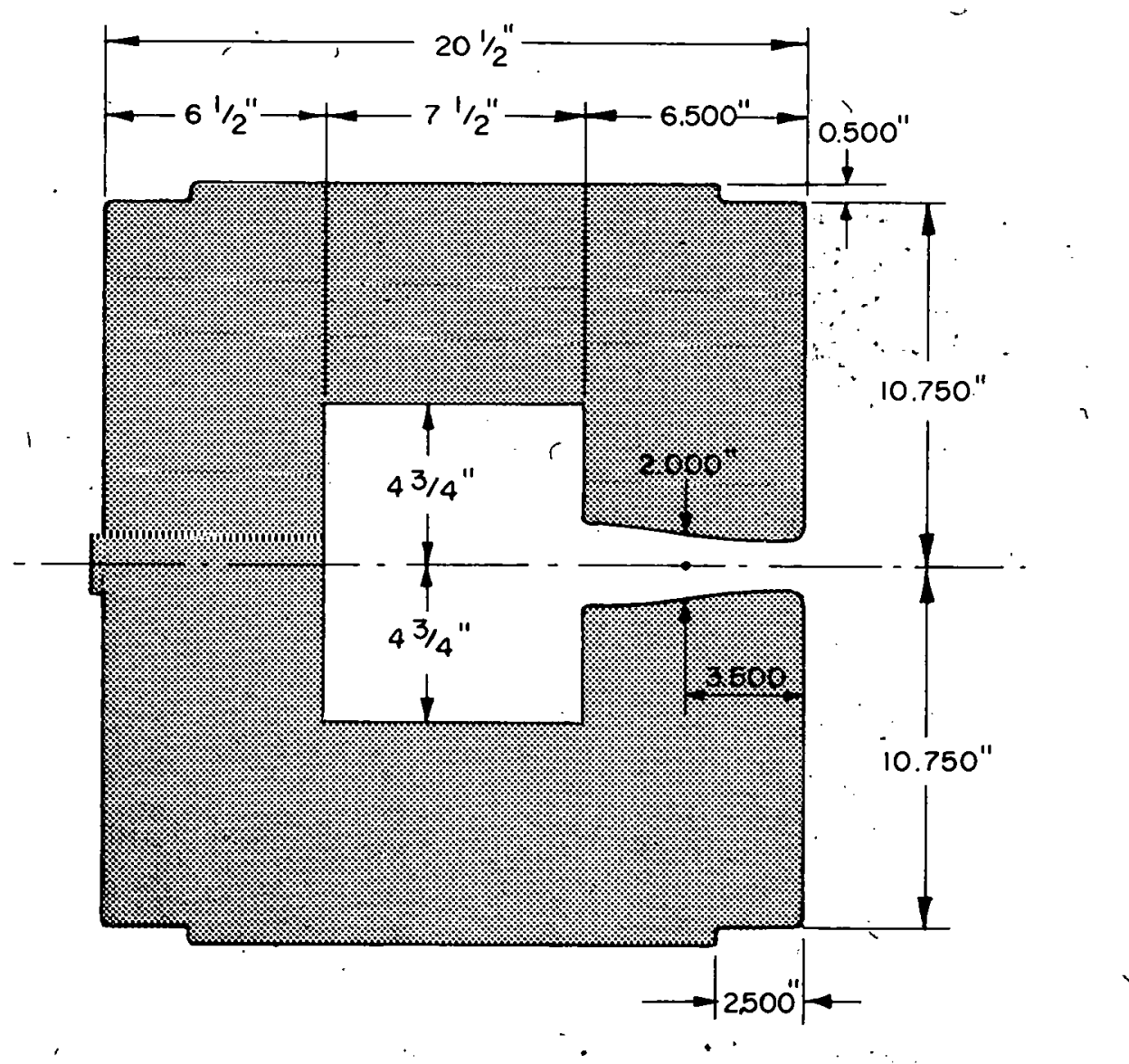

Fig. 4.4. Some key dimensions of a lamination of a closed-type magnet. 


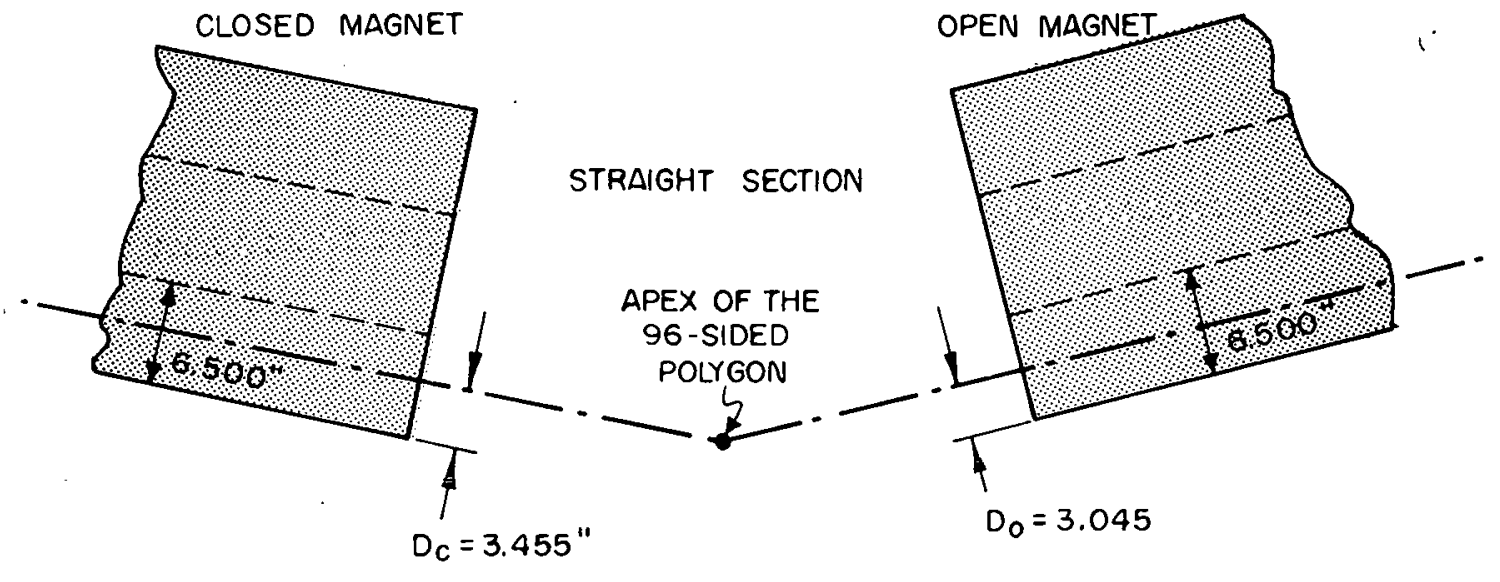

Fig. 4.5. Relationships of isomagnetic lines and front faces of magnets of closed and open type. The angle between the axes of the magnets has been exaggerated greatly. Until $7 / 12 / 61$ the distances $D_{c}$ and $D_{O}$ were $3.500^{\prime \prime}$ and $3.000^{\prime \prime}$ respectively, and the difference was $0.500 "$. Since that date the values have been 3.455" and 3.045", and the difference has been $0.410^{\prime \prime}$.

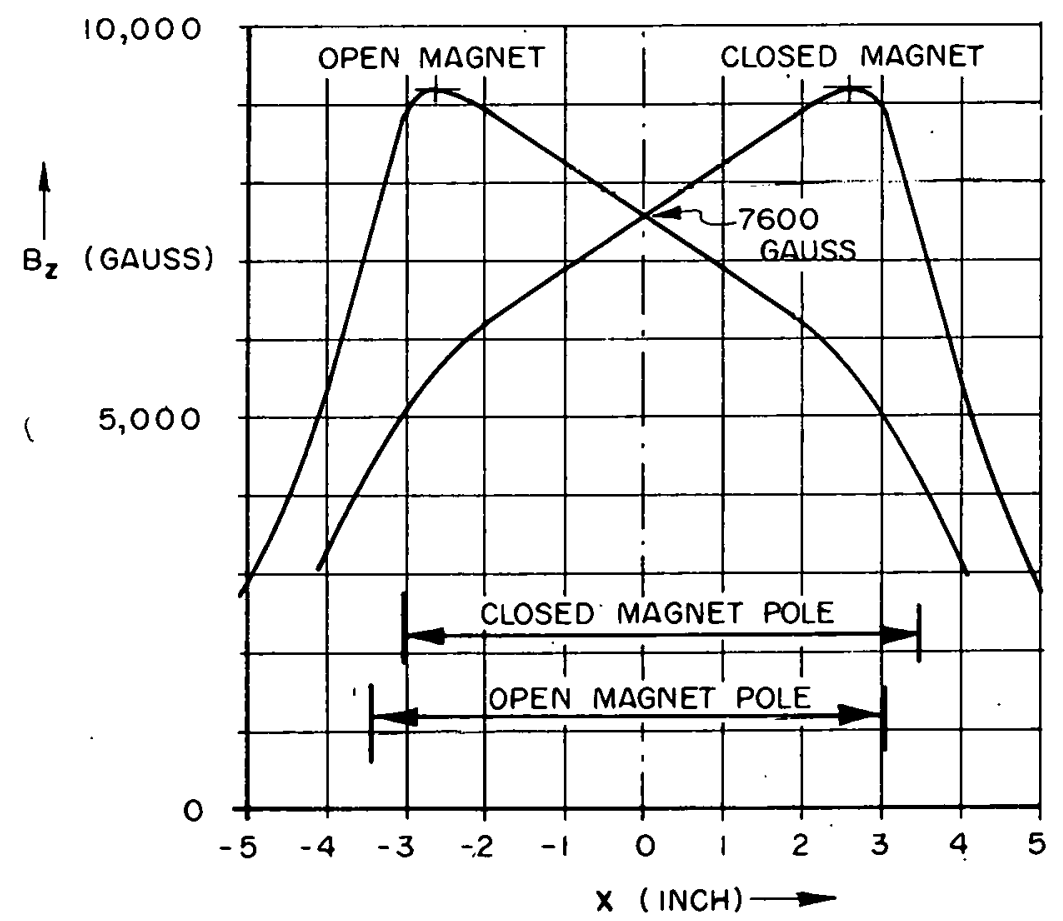

Fig. 4.6. Relationship between $\mathrm{B}_{z}$, the vertical component of magnetic field in the gap of one of the 48 magnets, and the radial position $x$ measured radially outward along the mechanical median plane, when the magnets are excited for 6-Bev operation. 
Chapter 5

Supporting and Aligning the 48 Magnets

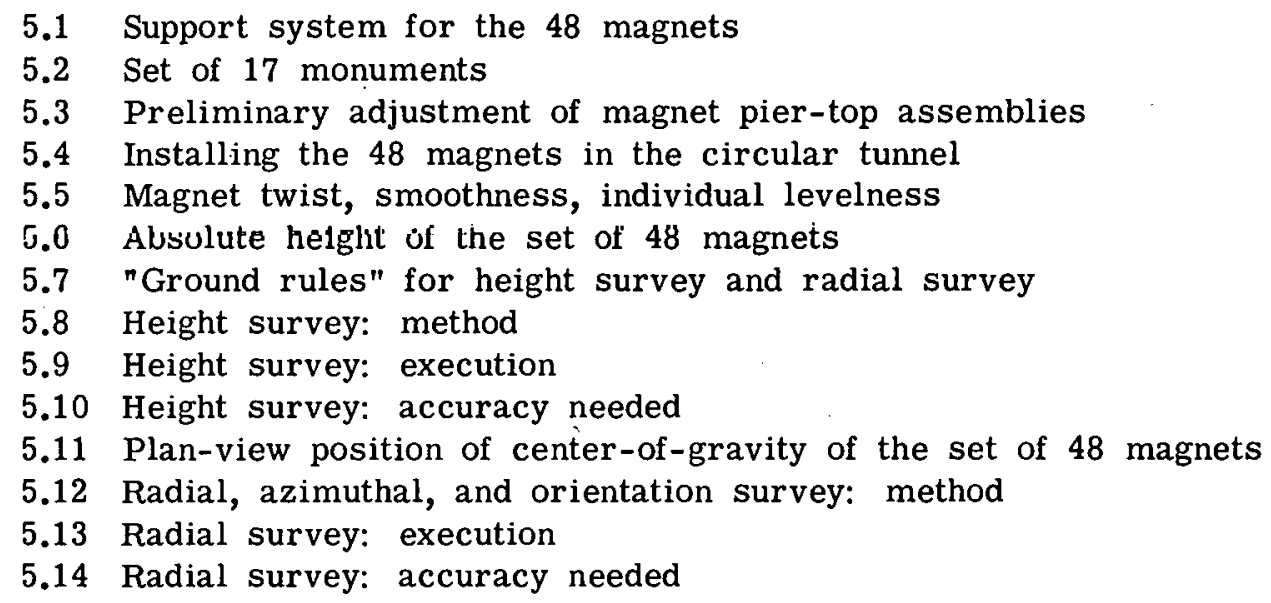

\subsection{Support System for the 48 Magnets}

Each of the CEA's 48 magnets rests on a $12-\mathrm{ft}$ girder, and each end of the girder rests on a pier-top assembly. The pier itself, capped by a welded-on stainless steel capping plate, is supported by two steel $\mathrm{H}$-section piles that extend approximately 30 to $40 \mathrm{ft}$. downward into undisturbed gravel.

Originally, the top of each girder was planed flat to within about $1 \mathrm{mil}$; then the guide rails, also flat to about $1 \mathrm{mil}$, were attached. The magnet core blocks were then set on these rails. When loaded by the 6-ton magnet and supported at the ends only, the girder sags very slightly, as indicated in the following chapter.

Each pier-top assembly includes a central $1 \frac{1}{2}$ " diameter vertical pin that engages a hole or slot in the underside of the girder, and includes also two Duff-Norton jacks that raise or lower the girder-end at the rate of 5.6 mils per revolution of the jack handle; total range of vertical travel is 1.75 inches. Also, two hydraulically actuated pistons and two rings of ball bearings are included, to facilitate radial or azimuthal motion of the girder. To bring the rings into play, one applies oil at a pressure of 200 psi. Two horizontal screws control the radial motion of the pier-top assembly. Azimuthal motion is controlled by screws at the ends of the girder. A depth gage may be used to record the radial and azimuthal positions. A precision of 1 mil can be achieved. The pier-top assembly as a whole is mounted perpendicular to the girder, and thus appears $3.75^{\circ}$ skew with respect to the pier's capping plate. 


\subsection{Set of Seventeen Monuments}

To assist in the original determination of the appropriate positions for the magnet support piers, a system of seventeen benchmarks, or monuments, was constructed. The centerpoint monument S-O is located in the Power Building basement, near the inner end of Tunnel 38; it is at elevation $20.12 \mathrm{ft}$. The other sixteen monuments (S-2, S-5, S-8, etc.) are situated in the circular tunnel and form a circle about Monument S-0; the nominal radius of this circle is $122.0000 \mathrm{ft}$., and the successive monuments are $22.5^{\circ}$ (i.e., $47.6020 \mathrm{ft}$.) apart. Each of the sixteen monument tops is quite low, namely $0.38 \mathrm{ft}$. below floor level (i.e., below Level $16.50 \mathrm{ft}$.). To gain access to a monument, one must remove a steel cover plate. The monument-top consists of a horizontal stainless steel plate in which there is a vertical conical hole (3.463/2.597 inches, to receive a $3.5000^{\prime \prime}$ dia. sphere). Each monument rests on an H-type steel pile driven about $30 \mathrm{ft}$. into undisturbed sand.

The detailed designs of the monuments and the method of determining their positions (with Keuffel and Esser Lovar precision tapes, Wild T-2 theodolite, and Farrand alignment telescope) are indicated in reports CEA-TM-43, CEA-TM-52, CEA-TM-53, and in Harry R. Feldman, Inc. report of 11/19/59. An accuracy of about 0.03 inch was achieved.

After having been used to locate the magnet piers and the 96 pier-top assemblies thereon with an accuracy of 0.03 to 0.06 inch, the monuments were not used further. Subsequent radial surveys have dealt just with the magnets themselves, relating to nothing except perhaps their collective center of gravity.

\subsection{Preliminary Adjustment of Magnet Pier-Top Assemblies}

After the monument system had been completed and the pler capplny plales liad been laid on top of the piers, the location of each capping plate was determined, relative to adjacent monuments, with the aid of the Wild T-2 theodolite and specially made spacer bars, or jig offsets, having net lengths of $56.335,165.991$, and 222.978 inches. The capping plate was then welded in place, and the pier-top assembly was installed and centered relative to the capping plate. This was done in 1959. The goal was to have the axis of , each $1 \frac{1}{2}$ " dia. pin of the pier-top assembly situated $118.0000 \mathrm{ft}$. from the accelerator centerpoint and equidistant from the corresponding pins of adjacent magnets, and to achieve a general rotational, or azimuthal, position such that Straight Sections 2, 14, 26, and 38 lay approximately on the centerlines of Radial Tunnels 2, 14, 26, and 38 respectively. It turned out (see a later paragraph) that the goal was achieved with gratifying accuracy. 


\subsection{Installing the 48 Magnets in the Circular Tunnel}

A total of 46 of the 48 magnets were installed in the circular tunnel in Oct. and Nov. 1960. Two were omitted pending completion of the inner experimental room adjacent to Tunnel 14.

In Dec. 1960 and Jan. 1961 many of the magnets' end-plates were removed and mounted on other magnets, to achieve greater overall uniformity of magnetic performance, and the magnets were rearranged in the circular tunnel in a more advantageous sequence.

On Feb. 1, 1961, the last two magnets were put into place, and the first radial survey was made immediately thereafter.

\subsection{Magnet Twist, Smoothness, Individual Levelness}

It was found that immediately after the magnets were assembled and installed in the circular tunnel, some of the magnet girders exhibited a small amount of twist. In extreme cases, if the four supporting jacks were at exactly the same height, the girder would rest on three of these and a gap of a few mils would appear between the girder and the fourth jack. Within a few days, however, the magnet (the core blocks, presumably) would "relax" and bear with almost equal force on all four jacks.

The upper shoulders of the magnets. were found to be smooth enough so that moving the small three-legged optical target to various locations (on the shoulder) within 6 inches of a given location would change the height of the target by only about 2 mils $\mathrm{rms}$.

Inicially, one corner of a given magnet might be found to be very different in height from the other corners. First priority was given to bringing all four corners to the same height within 2 mils. Only after this had been done was the height of each magnet compared with that of a neighboring magnet.

\subsection{Absolute Height of the Set of 48 Magnets}

The original choice of nominal height of the mechanical median plane of the synchrotron (i.e., nominal plane of the orbit) was 60.000 inches above floor level, i.e., at Level $21.50 \mathrm{ft}$. (defined in Chap. 3). In Nov. 1960 the magnet heights were adjusted so as: to make the mechanical median plane just 60 inches higher than the floor region directly beneath the upstream end of the Magnet 48 girder.

However, on $12 / 15 / 60$ the entire set of magnets. was raised $250 \mathrm{mils}$, because it had been learned that some areas. of the floor (e.g., some areas that were to support the 
Main Shielding Wall) were $1 / 2$ inch higher than the region beneath Magnet 48 . Thus the nominal height became 60.250 inches.

With the set of 48 magnets at this height, the average protrusion of shaft of the Duff-Norton jacks is about $3 / 4$ inch, meaning that the jacks are comfortably close to the middle of their available 1.75 inch range of motion.

It was desired, of course, that the accelerator plane be horizontal - for convenience in surveying and in order that the emerging beams of photons and electrons would be approximately horizontal. The height survey, described in the following paragraphs, was made in such a way as to insure that the orbit is indeed horizontal.

\section{7 "Ground Rules" for Height Survey and Radial Survey}

Every effort was made, in planning the survey procedures, to make it feasible to make the surveys (1) fast, to minimize machine tie-up time, (2) without the need for dismantling any accelerator components, such as linac drift tubes or waveguide riser sections, (3) without use of lines of sight exceeding $50 \mathrm{ft}$. (which invite large errors due to temperature gradients and refractive index gradients in the air), (4) without the need for measuring angles, and (5) accurately enough, especially with respect to the 6 th, 7 th, 17 th, and 18th harmonics in magnet height. (The special importance of these harmonics is indicated in later paragraphs.)

\subsection{Height Survey: Method}

To find the relative heights of the magnets, the surveyor compares Magnets 48 and 1. Then he compares 1 and 2, then 2 and 3, and so on, obtaining 48 values of heightdifferences. From these, and after computing and distributing the closing error, he computes the 48 values of height relative to Magnet 48.

To compare, say, Magnets 3 and 4, he employs a Wild 42X Type N-3 telescopic level. This is situated on top of an extra-tall, extra-heavy tripod situated equidistant from the two magnets in question and about $4 \mathrm{ft}$. radially outward from the ring of 48 magnets. The optical target employed consists of a small bit of Keuffel and Esser Wyteface scale mounted on a tiny three-legged holder. The target is mounted first on Magnet 3 , then on Magnet 4. In each case it is situated on the top, radially outer shoulder of the magnet, at a location about 4 inches upstream from the gap between the two sets of core blocks; the position is established quickly and reproducibly with a small loosely fitting fixture. 
The precision with which the surveyor makes his settings and readings is about $1 / 2$ mil. To eliminate certain kinds of cumulative error, the optical target, once set on Magnet 4, is left untouched until both readings on Magnet 4 have been made, i.e., the reading relative to Magnet 3 and the subsequent reading relative to Magnet 5 .

\subsection{Height Survey: Execution}

Height surveys were made on eight occasions between $9 / 16 / 60$ and $3 / 1 / 64$. Typically, the survey was marle in 4 hours, by onc man. The clusing error was 3 mils.

At the conclusion of a typical survey, the magnet heights were readjusted as required - in about two hours.

Analysis of the survey results shows that, in the first few months of 1961, a typical magnet changed in height by about 1 mil per month, relative to the set of 48 magnets. During 1962, the change was at about half this rate, and during 1963 the change was slightly smaller still. By $3 / 1 / 64$ the heights had been left unadjusted for 17 months, and the vertical distortions of the orbit at high field were so small that the decision was made to leave the heights unadjusted for some time to come.

\subsection{Height Survey: Accuracy Needed}

Bearing in mind that the design value of vertical betatron oscillation is about 6.4, one appreciates that if the magnet heights varied so that, around the entire ring, there were 6 or 7 "waves," or were $(24-6)$ or $(24$ - 7) waves, the orbiting electrons would be found to exhibit large vertical betatron oscillations. In other words, the 6th, 7th, 17th, and 18th harmonics in magnet height are especially harmful. Other harmonics are far less harmful. The situation has been analyzed and reported by $\mathrm{K}$. W. Robinson in his memoranda of 4/15/60 and 5/19/60. In summary: an $\mathrm{rms}$ error of $0.5 \mathrm{mil}$ in the individual magnet-height comparison is so small that the heights can be adjusted accurately enough that the maximum amplitude of vertical betatron oscillation is not likely to exceed about 15 mils; furthermore, a five-times greater surveying error would still probably be tolerable.

Actual measurements on orbit distortions have been made with the aid of pick-up coils discussed in another chapter. The measurements indicate that the magnet heights are sufficiently uniform by a wide margin.

It seems likely that the uniformity of height will continue to be adequate even if the heights are not readjusted for 2 or 3 years. 


\subsection{Plan-View Position of Center of Gravity of Set of 48 Magnets}

Because the magnets were mounted in the ring after the cap plates on the piers had been welded in place, the surveyor's goal - in making the first radial and azimuthal surveys of the magnets themselves - was to arrive at positions compatible with these plates and indeed such that each magnet would find itself somewhere near the middle of its available range $( \pm 3 / 4$ inch) of horizontal adjustment. It turned out that the cap plates lay very close to the ideal positions (within $1 / 16$ inch in most instances), and consequently it was indeed possible to correct the magnet positions and at the same time keep each magnet near the middle of its range.

\subsection{Radial, Azimuthal, and Orientation Survey: Method}

The so-called radial survey of the 48 magnets entails measurement not only of the radial positions of each magnet but also the azimuthal positions (i.e., along the arc of the circle) and the orientations. The telescope used and optical targets used are mounted - not at the centers of the magnets - but on "outrigger" fixture plates extending about $3 \mathrm{ft}$. radially outward from the magnet in order to provide 50-ft.-long clear lines of sight; accordingly, none of the three parameters is orthogonal to any other, and none can be evaluated adequately without some knowledge of the others.

The method of determining the radial position, azimuth, and orientation of each mag-. net conforms to all the ground rules mentioned in a previous paragraph. It consists of dealing with one set of four contiguous magnets at a time. By making forward sights among the group of four, and by making backward sights also as a check, the surveyor.obtains. data as to the radial positions and orientations of the second and third magnet relative to the first and fourth. Tapes are used to measure the relative azimuths. The process is repeated for all 48 sets (overlapping sets). Then a computer operates on these "relative". data and computes the absolute radial and azimuthal motions required for each end of each magnet. (The appraisal of the radius of the circle is a separate subject, and is discussed in a later paragraph.)

Fig. 5.1 shows the plan of survey for the set comprising Magnets 48, 1, 2, 3. To hold the Farrand 60X alignment telescope and the optical target, the surveyor employs a set of four specially designed fixtures. Each fixture is a kind of horizontal platform that extends radially outward from the magnet and bears firmly against the front guide rails in clean and definitive manner. (Since the widths of the guide rails are alike within 1 mil, 
affixing the fixtures to the rails is equivalent to affixing them to the core blocks themselves.) Each fixture contains two built-in, symmetrically located depth gages, which are used in mounting the fixture symmetrically. on the magnet - symmetrically with respect to the vertical radial plane through the point where the centerlines of the two guide rails intersect.

Each fixture has an outer hole, or station, for locating a telescope or optical target at one end of the 47-ft.-long line-of-sight, and each has ịner holes fọ locating an optical target. The holes were drilled in the fixtures at such locations that when the magnet positions are exactly correct in every way, the holes concerned in any one sighting lie exactly along the line-of-sight, i.e. on a straight line. Consequently there is nothing to measure, nothing to record except "zero." If there are lateral deviations, these are small and can be measured directly, instantly, in terms of mils, by means of the optical micrometer contained within the alignment telescope. The fixtures contain inner wingstation holes for holding targets when the surveyor wishes to appraise the orientation of a magnet. If the orientation and position are correct, the line-of-sight passes through the target when it is placed successively in the various wing stations, and there is nothing to record except zero.

Necessarily, all four. fixtures have different geometry. This is unavoidable in view of the upstream-vs.-downstream choice of position of a given magnet of the group of four, and the open-vs.-closed choice of type of magnet. It will be remembered that the original plan called for placing the closed magnets so that their guide rails were 0.500 . inch farther outward radially than the guide rails of the open magnets. Accordingly the fixtures for closed magnets were designed to be 0.500 inch shorter, radially, than those for open magnets. (On $7 / 12 / 61$ the displacement was changed from 0.500 to 0.410 inch, for reasons explained in $\mathrm{H}$. Winick's $7 / 8 / 61$ memorandum.)

The azimuthal part of the survey employs the same fixtures, but no optical instruments; it employs, rather, an invar steel tape made specially by Keuffel and Esser. With: this tape the surveyor, measures the distance between the central inner stations of the fixtures attached to the two. inner magnets. Special mechanical targets are used, and the tape is read with the aid of a hand magnifying glass to the nearest mil. The design of the accelerator is such that the distance should be $15.6853 \mathrm{ft}$., as is shown in Drawing C-WS-3/18/60.

A. Bendix G-15 computer is then used to process the mass of relative data on radial position, azimuthal position, and orientation, and compute the necessary radial and 
azimuthal motions required of each pier-top assembly in order to make the plan-view positions of the 48 magnets correct in every respect. The program was devised just prior to $2 / 23 / 61$. The difficult part of the task was to find the appropriate Green's function.

Radius of the circle: Even though the magnets may lie on a perfect circle and have the correct angular positions and orientations, the radius of the circle may not have the correct value. To find whether it has the correct value, the surveyor adds the 48 values (each nominally $15.6853 \mathrm{ft}$. as indicated above) of distance between adjacent magnets' fixture plates. If the sum is too large by, say, one part in $10^{6}$, he moves all magnets radially inward so as to decrease the radius by one part in $10^{6}$.

\subsection{Radial Survey: Execution}

Three radial surveys were made, on approximately $2 / 1 / 61,4 / 2 / 61$, and $11 / 14 / 61$. On about 2/18/63 a radial survey of half the ring was made. Each full survey required about 25 hours of survey work in the circular tunnel, four hours of paper work, a 7-hour computing machine run, and 8 hours for readjusting the plan-view positions of the magnets.

No noteworthy difficulties were encountered in these surveys. The back-sight data agreed with the forward-sight data within about 2 mils. Each reading of target displacement from line-of-sight was determined with a precision of $1 \mathrm{mil}$ and an accuracy of 2 mils (or perhaps 4 mils on those occasions when disturbing air currents and temperature gradients existed). No interference was caused by the linac drift tube; no part of the accelerator had to be disturbed; normal maintenance work in the tunnel continued.

The first survey showed that the .typical end of a typical magnet had an incorrect plan-view position by about 70 mils (occasionally as much as 150 mils), yet the computer output data showed correctly how to eliminate virtually all of the error. This is evidenced by the fact that the second survey, made two months later, showed that only negligible errors remained, e.g., radial errors of about 5 mils $\mathrm{rms}$, and even these must be attributed in part to changes that occurred after the first survey had been completed. By the time the third radial survey was made (7 months later), the radial error had increased to about 10 mils $\mathrm{rms}$; the subsequent readjustments reduced this error to about 4 mils rms. In the half-survey made in Feb. 1963 (14 months later), it was found that the radial positions had changed only negligibly - specifically, by about 3 mils rms.

In order to correct errors found in the mean radius of the ring, the magnets were moved 180 mils outward after Survey \#1, 60 mils inward after Survey \#2, and left unchanged after Survey \#3 (although the radius then appeared 45 mils less than the nominal value). 


\subsection{Radial Survey: Accuracy Needed}

Estimates made in advance indicated that errors of the magnitudes implied above would be entirely acceptable. Again the crucial errors are those contributing to the 6th, 7 th, 17th, and 18th harmonics in radial position, since the design value of the frequency of horizontal betatron oscillations is the same as that of the vertical oscillations, namely about 6.4. It was estimated that there was only about a $1 \%$ chance that the maximum horizontal displacement of the equilibrium orbit would exceed 25 times the $\mathrm{rms}$ error in an individual measurement.

Measurements made of radial distortions present in the orbit of $4 \mathrm{Bev}$ and $6 \mathrm{Bev}$ electrons during the last four months of 1962 showed that the horizontal distortions were very small in all 16 of the straight-section tanks that contained pick-up coils for sensing orbit distortions. In no tank did the displacement exceed $1 / 3$ inch.

\section{References:}

D. D. Jacobus: CEA-TM-43, CEA-TM-52, and CEA-TM-53 re monuments.

K. W. Robinson: Memoranda of $4 / 15 / 60$ and $5 / 19 / 60$ re accuracy requirements in magnet surveys. Also Bendix G-15 computer programs of 2/23/61 re clockwise radial survey and counterclockwise radial survey.

W. A. Shurcliff: Memorandum of $11 / 7 / 60$ re monuments. Also CEA-TM-64 and CEA-TM-79 and memoranda of $5 / 24 / 61,5 / 25 / 61,9 / 26 / 61$, and $3 / 16 / 62$ re height surveys. Also memoranda of $12 / 16 / 60,2 / 28 / 61,3 / 15 / 61,12 / 4 / 61,3 / 7 / 63$ re radial surveys. 


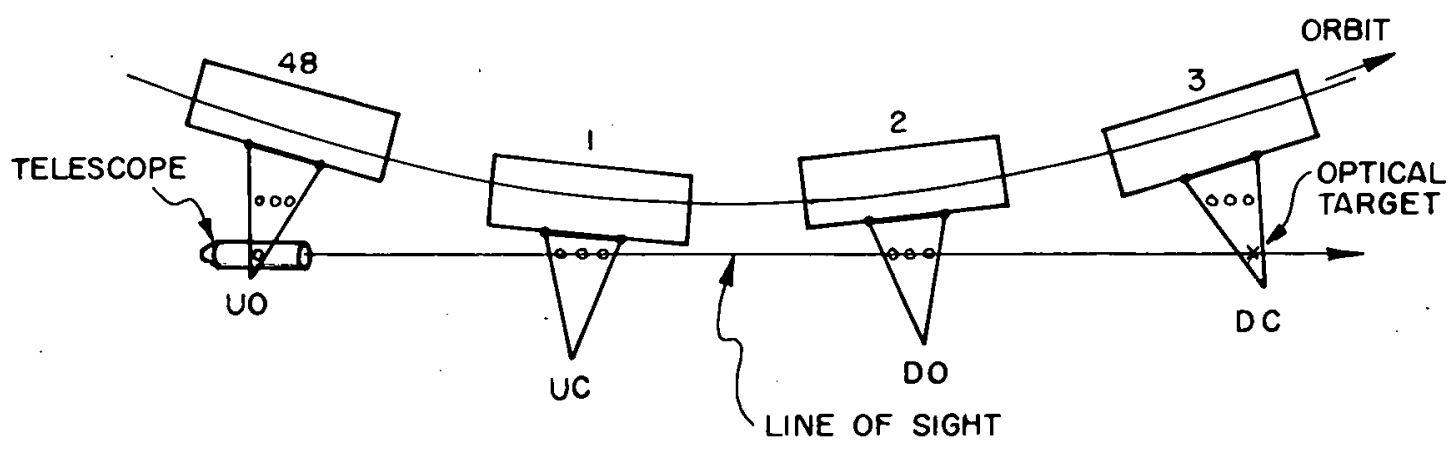

Fig. 5.1. Schematic, plan-view diagram of the four fixture plates in place on a group of four magnets. Each plate contains holes for locating a telescope or target. Two plates are for use on open (O) magnets and two for use on closed (C) magnets; two are for use at an upstream (U) location and two for use at a downstream (D) location. Thus no two are alike. 


\section{Chapter 6}

\section{Powering the 48 Magnets}

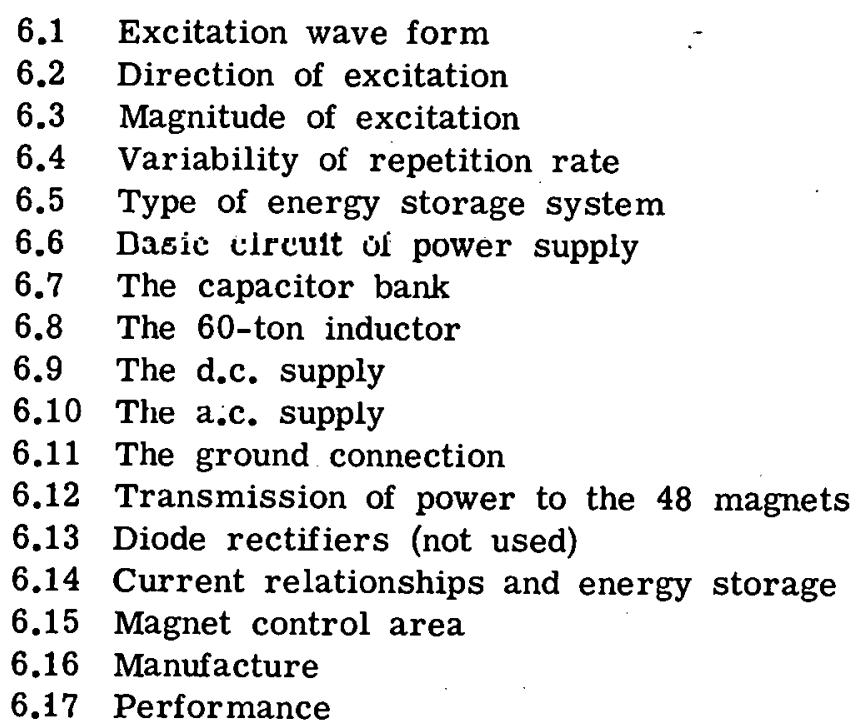

\subsection{Excitation Wave Form}

Chapter 4 explains the decisions to use 48 magnets and a repetition rate of b0 cycles/sec, but says little about the excitation wave form. Early in the design of the accelerator the designers proposed to use full-biased excitation, pictured in Fig. 6.1 and specified by:

$$
I(t)=I_{\text {ave }}(1-\cos 2 \pi f t)
$$

where $I(t)$ is the instantaneous current in the magnet coils, $I_{\text {ave }}$ is the: average current; $f$ is the frequency or repetition rate $(60$ per $\mathrm{sec})$, and $t$ is time. Obviously the current $I(t)$ varies sinusoidally about the average value and the least value is zero; that is, the current just fails to reverse.

As explained in CAP-12, CAP-12a, and CEA-59, such excitation has the following. advantages over unbiased (simple sine-wave) excitation:

a. The field strength at the start of the cycle, when injection is to occur, varies extremely slowly; that is, $\mathrm{dB} / \mathrm{dt}$ is very small. Consequently, the tolerance on timing of injection is reasonably large, being perhaps $3 \mu \mathrm{s}$

during 6-Bev operation and perhaps 10 or $20 \mu \mathrm{s}$ during 1-Bev operation. 
Also, small $\mathrm{dB} / \mathrm{dt}$ implies small eddy currents and small distortion of the orbit. In addition it allows time for a reasonably large fraction of the freshly injected electrons to settle into stable orbits.

b. The hysteresis losses in the magnets are smaller and hence the magnet power requirement is reduced.

c. The energy demand of the freshly injected electrons is much smaller and therefore the rf buckets (defined in Chap. 3) are longer in phase and a larger fraction of the electrons can be captured into stable orbits.

d. The rate of change of electron energy near the end of the acceleration interval is smaller and therefore a longer spill time may be used; for a given permissible range of spill-energy the spill lime is longer by $\sqrt{2}$.

It was later decided that the amplitude of the a.c. component of excitation should be increased enough so as to slightly exceed the average current (biasing current, or d.c. component) and produce a brief interval in which the direction of the current reverses. By measuring and controlling this interval, an operator can maintain a fixed relationship between the two components. The decision was made to use an a.c. component having an amplitude $1.8 \%$ greater than the d.c. component, which produces a reversal interval of about 1 millisecond. Thus:

$$
I(t)=I_{\text {ave }}[1-(1.018) \cos 2 \pi f t]
$$

\subsection{Direction of Excitation}

Because the electrons are to travel around the ring in counterclockwise sense, as viewed from above, the field in the magnet gap must be upward and the conventionally defined current in the individual coil must be counterclockwise.

\section{$\underline{6.3 \quad \text { Magnitude of Excitation }}$}

A 1963 analysis that took account of the actual geometry of the equilibrium orbit of $6.00 \mathrm{Bev}$ electrons and the actual properties of the 48 magnets showed that, to hold the electrons in this orbit, each magnet must provide, at the isomagnetic line within the. gap, a field strength of 7620 gauss. Such a field is achieved when the instantaneous current in the individual coil of the magnet is 761 amps. To achieve such a current one may employ a d.c. component of 377 amps and an a.c. component having a peak value of 
(377)(1.018) or $384 \mathrm{amps}$ and an $\mathrm{rms}$ value of $271 \mathrm{amps}$. The extreme values of total instantaneous current are then $(377+384)$ or 761 amps and $(377-384)$ or -7 amps. The rms current is 465 amps.

\subsection{Variability of Repetition Rate}

The designers decided to design the power supply so that operation at any frequency within $1 \%$ of the design frequency $(60$ cycles $/ \mathrm{sec}$ ) could be accommodated. They believed that the power supply might be operated as an oscillator; operating at its natural resonant frequency, and anticipated that this frequency might differ from the nominal frequency (the line frequency) by as much as $1 \%$.

Actually, in nearly all operation through $3 / 1 / 64$, the frequency was tied to the $60-$ cycle line frequency.

Early in the course of the design, the designers entertained the possibility of operating, at will, at 60 cycles/sec or 30 cycles/sec, for reasons explained in CEA-59. In 1958 the latter frequency was dropped from consideration.

\subsection{Type of Energy Storage System}

Because the peak amount of energy required for $6-\mathrm{Bev}$ excitation of the magnets is so large (exceeding 460,000 joules), and because only about $6 \%$ of this is dissipated in the magnets, considerations of economy of power utilization dictated that, between intervals of peak excitation, the remaining energy be stored. In some accelerators, notably those having repetition rates as low as one pulse every few seconds, massive flywheels have been used as storage devices. But when the rate is to be as high as 60 per second the designers have the opportunity of choosing an all-electric storage system, that is, a resonant system consisting of inductors and capacitors. The designers made estimates (see CAP-12, CAP-12a, CEA-16, CEA-59) indicating that, for this accelerator, electrical storage would entail a lower capital investment and would provide greater versatility and greater reliability. Also, electrical storage avoids the high noise levels associated with flywheels. Accordingly they decided: on electrical storage. The system adopted employs a set of 24 capacitor banks and a unique 60-ton inductor. These are described in later sections.

\subsection{Basic Circuit of the Power Supply}

Fig. 6.2 is an abbreviated circuit diagram of the power supply for the magnet coils. A full diagram would show 24 capacitor banks, 24 secondary coils, and 24 groups of mag- 
net coils. (Each of these last groups includes the coils of two adjacent magnets, such as Magnets 2 and 3.) The 24 secondary coils are part of the 60-ton air-gap inductor discussed in a later section.

The logic underlying the circuit is easily understood by referring to Figures 6.3, 6.4, 6.5. It is explained also in CEA-59, CEA-68, CEA-74, CEA-TM-86.

Fig. 6.3 shows a greatly simplified circuit containing just capacitors and inductors; each inductor represents one group of magnet coils. Obviously the current can oscillate in each portion of the circuit. And if all the capacitances are alike and all the inductances are alike, the oscillations in all portions can have the same frequency, same phase, and same magnitude. The energy may be thought of as flowing back and forth from inductors to capacitors.

In Fig. 6.4, choke-coils have becn added, by-passing the capacitors, to permit direct current to flow through the entire train of inductors; thus biased excitation is made possible.

In Fig. 6.5, primary coils for initiating and maintaining the oscillations have been added.

By rearranging this diagram and including appropriate terminals, one arrives at Fig. 6.2. The 24 primary windings are powered in parallel, and the 24 secondaries are connected in series.

Whether a given pair of secundary windings is to be rogarded as serving the two adjacent upstream magnets, the two adjacent downstream magnets, or one adjacent upstream magnet and one adjacent downstream magnet is, of course, arbitrary. The same applies to a given capacitor bank. The symbols used at CEA for the various magnets, secondary windings, and capacitors are such that, for example, the pair of secondary windings called \#3 and the capacitor bank called \#3 are situated between Magnets 3 and 4, and thus may be said to serve Magnets 3 and 4. Such an assignment of symbols seems especially appropriate when one remembers that the upstream end of Magnet 3 and the downstream end of Magnet 4 are nominally at "a.c. ground potential," so that the loop circuit comprising secondary windings \#3 and capacitor bank \#3 is, in a sense, a selfsufficient unit.

The circuit as a whole may be likened to that of a self-excited Class-C oscillator with a natural frequency of $60 \mathrm{cycles} / \mathrm{sec}$ and a $Q$ of about 80 . One can change the frequency as much as $1 \%$ by changing the capacitances of the capacitor banks, and one could 
make a much greater change by altering the magnet connections and the connections of the secondary windings of the inductor.

During 6-Bev operation of the synchrotron, the losses in the entire magnet supply, including not only the magnet coils and inductor but also the equipment for supplying d.c. to the coils and a.c. to the inductor primaries, amount to 1400 to $1500 \mathrm{kw}$. Approximately $3 / 4$ of this may be assigned to the a.c. component and 1/4 to the d.c. component.

The locations, magnitudes. and phases of energy storage in the circuit are discussed in a later section, after the designs of the individual circuit components have been described.

\subsection{The Capacitor System}

The capacitor system consists of 24 banks each of which has a maximum available capacitance of $180.5 \mu \mathrm{f}$ and a voltage rating of $7.6 \mathrm{kv} \mathrm{rms}$. The bank includes 76 individual capacitors connected in series-parallel arrangement. Each of these 76 is a Westinghouse Outdoor Type FP, Style 2K1272, capacitor intended for use with single-phase 60cycle current; each is rated at $9.5 \mu \mathrm{f}, 3.8 \mathrm{kv} \mathrm{rms}$, and $50 \mathrm{KVA}$, and is provided with a current-limiting fuse.

Four of the 76 capacitors can be connected or disconnected individually, by remote control; thus the operator can compensate for the small decreases in capacitance that result from warm-up of the capacitors. (The typical change in capacitance that occurs as a result of warm-up is of the order of $2 \%$; such a change produces a $1 \%$ change in resonant frequency of the magnet powering circuit.) Normally 64 to 68 of the capacitors are in use, and the rest are left unconnected; thus the actual capacitance of the bank is about 152 to $161.5 \mu \mathrm{f}$. Perhaps $155.5 \mu \mathrm{f}$ is typical.

If all of the (24)(76) or 1824 individual capacitors were connected in parallel, the total capacitance would be $(1824)(9.5 \mu \mathrm{f})$ or $17,328 \mu \mathrm{f}$, with a voltage rating of $3.8 \mathrm{kv} \mathrm{rms}$. Because of the series-parallel arrangement and because, ordinarily, a few of the capacitors are left unconnected, the actual working capacitance is about $3700 \mu \mathrm{f}$ and the voltage rating is $7.6 \mathrm{kv}$ rms.

Each capacitor bank occupies a region about $15 \mathrm{ft}$. long, $4 \mathrm{ft}$. wide, and $8 \mathrm{ft}$. high, and includes four rows: two at waist height and two at head height. Each row contains 19 individual capacitors; all the capacitors in the upper rows are connected in parallel and all those in the lower rows are likewise connected in parallel, but the two groups 
(upper and lower) are connected in series. The steel framework holding the 76 capacitors is insulated from ground. For further details as to the individual bank and its framework, see "CEA Resonant Capacitor Specifications" dated 7/17/58, Rev. 8/14/58.

Together, the 24 banks occupy a $45 \times 50 \mathrm{ft}$. outdoor yard NE of the Power Bldg. The 24 frameworks are electrically insulated from one another.

Since the biasing current that runs through the train of 24 magnet pairs and 24 choke coil secondaries is large, the potentials $\mathrm{P}_{1}, \mathrm{P}_{2}, \mathrm{P}_{3}$, etc. of the midpoints of successive capacitor banks differ progressively and considerably. Nominally the "steps" $\left(P_{2}-P_{1}\right),\left(P_{3}-P_{2}\right)$, etc., are identical. But in practice some variability occurs. To minimize this a parallel potentiometric circuit is provided. It includes a d.c. supply and a train of twenty-four 100-ohm resistors, and the connections to the various midpoints (and likewise to the steel frameworks) are made by twenty-four 10,000-ohm resistors. During 6-Bev operation of the synchrotron, the rms voltage across one capacitor bank is slightly less than $7 \mathrm{kv}$.

\subsection{The 60-Ton Inductor}

The single most massive component of the entire accelerator is the 60-ton inductor, or choke, of the magnet power supply, pictured in Fig. 6.6 and described in detail in CAP-12, CAP-12a, CEA-59, CEA-66(2), CEA-68, CEA-74, CEA-76, CEA-TM-32, CEA-TM-39, CEA-TM-59. The device, situated in the Power Bldg. Basement, performs many functions, including:

a. providing, via the secondary coils (choke coils), a path for the direct current that flows through all 24 groups of magnet coils in series;

b. providing primary coils whereby pulses of power may be fed to the circuit segments to maintain the 60-cycle oscillations;

c. providing air-gaps (filled, actually, with plates of Micarta) in which large amounts of energy may be stored;

d. providing considerable "flux sharing" of adjacent secondary coils to help insure that the a.c. in each group of coils is almost identical to that in neighboring groups, in magnitude and phase.

As indicated in Fig. 6.6, the heart of the inductor is the set of 12 four-ton core blocks, arranged in circular array. The individual core block, about 61" high and $31^{\text {" }}$ in 
radial dimension; consists of a vertical stack of six horizontal, trapezoidal-shaped, 10-inchhigh assemblies, each made of hundreds of 0.014 -inch-thick horizontal laminations of grainoriented transformer steel, the grain orientation being in the direction of the azimuthal flux. The laminations are bonded with an insulating material known as Cycleweld C-6. At the top and bottom of the core block there is a water-cooled copper plate designed to. keep the flux lines nearly horizontal near the outer edges of the core block, and thus eliminate the danger of overheating here. Each core block is cooled by air that is circulated at high speed in four vertical passages. (Until the fall of 1963 it was cooled by silicone oil, as explained in a later section.)

The 12 core blocks rest on rubberized fabric (Fabreeka) pads which rest in turn on 8-inch-thick maple blocks attached to a stainless steel foundation ring. The core blocks are $2 \frac{1}{2}$ " apart, and these air gaps are filled with $2 \frac{1}{2} "$-thick plates of Micarta; this tough plastic has good dielectric properties and hence provides excellent storage of electromagnetic energy; also it is strong enough to resist the tremendous. compressive forces that arise when the core blocks are energized and attract one another strongly.

The circular array of core blocks and Micarta plates is held together not only by these compressive magnetic forces but also by radial tie rods acting via wooden pressure. blocks. The system is compliant enough to allow several mils of radial "breathing" that results from the full-biased 60 -cycle powering:

Embracing each core block is a single coil, which includes one primary winding and four sets of secondary windings. (This applies to coils that were rebuilt in 1963 according to an improved design. The original design called for two primary. windings.) The primary winding serves two pairs of magnets; it includes 20 turns of copper conductor, and contains about $50 \mathrm{lbs}$. of copper. The d.c. resistance is $0.060 \mathrm{ohm}$.

Two of the secondary windings are connected in series with one another and with one pair of magnets; the other two serve another pair of magnets. The d.c. resistance of a series-connected pair of secondary windings is $0.0285 \mathrm{ohm}$ and the effective inductance, medsured with the coils in place in the completely assembled inductor, is 132.8 millihenries. Each of the four secondary windings includes 24 turns. In the set of four secondary. windings there are about 1600 lbs. of copper. The conductor is of pierced type and is cooled by circulating water.

The coupling between the secondary windings of the 60-ton inductor varies from almost unity for windings included in a single coil and embracing. a single core block, to 
about 0.18 for radially opposite windings, according to tests made on $3 / 31 / 61$. The common component of flux linking the windings acts to reduce voltage variations among the windings.

The coils are held in place by compliant clamps that allow them to vibrate independently of the core blocks.

Just above the inductor are several concentric arc-shaped bus bars carrying current to or from the terminals of the coils.

The inductor is situated at the center of an 18-ft.-diameter vertical cylindrical wall of concrete blocks. The diameter was chosen so as to provide a radial separation, between core block and wall, of just one-fourth of a wavelength of 60-cycle sound, i.e., a separation of about $4 \mathrm{ft}$. This insures that the principal sound waves radiating outward from the circular array of core blocks will be reflected back to the core blocks just onehalf cycle out of phase, causing such a poor acoustical impedance match that, in fact, little 60-cycle sound is radiated. Before the wall was built, the sound levels in nearby work areas were almost intolerable.

During $\dot{6}-\mathrm{Bev}$ operation of the synchrotron the peak field in the core blocks is about 15 kilogauss.

Tests made in Jan. 1964 showed that after the inductor has been in steady operation for 12 hours at 5-Bev level the temperature rise in the $24 \mathrm{gal} / \mathrm{min}$ flow of cooling water for the coils is 31 Fahrenheit degrees, the temperature $1 \cdot$ ise in the $00 \mathrm{gal} / \mathrm{min}$ flow of cooling water for the end plates is 5 degrees, and the temperature rise in the $1100 \mathrm{cu} \mathrm{ft} / \mathrm{min}$ flow of cooling air for the core blocks is 33 degrees. The corresponding heat dissipations are 110,80 , and $12 \mathrm{kw}$ respectively. The highest temperature measured at the surface of a core block during $5-\mathrm{Bev}$ operation was $145^{\circ} \mathrm{F}$.

\subsection{The d.c. Supply}

The d.c., or biasing, current for the 48 magnets is provided by Power Supply No. 2, built by Westinghouse Electric Corporation. The circuit diagram is shown in CEA-TM-86. See also CEA-16, CEA-59, and the Westinghouse Instruction Manual. The device has a current rating of 500 amps at 1200 volts, implying a power of $600 \mathrm{kw}$. Tests made in March 1961 showed that as much as 750 amps may be supplied for brief periods. During 6-Bev operation of the synchrotron, Power Supply No. 2 furnishes 377 amps at about 800 volts, or a power of about $300 \mathrm{kw}$. 
The power is derived from a $13.8 \mathrm{kv}$, three-phase, 60-cycle line. Power from this line is transformed by means of an auto-transformer and a rectifier-type transformer to an appropriate voltage and is applied to a three-phase, bridge-type rectifier employing six ISE-3 ignitron tubes. By varying the time (i.e., phase) of firing of these tubes, the operator can achieve any desired output from 60 to 600 amps.

A two-stage LC-filter reduces the 360-cycle voltage-component in the output by about $80 \mathrm{db}$. The filter includes two 33-millihenry inductors and appropriate capacitors. The inductances are provided by two large magnets that were prototype-variants of the 48 magnets used in the ring.

The transformers for Power Supply No. 2 are situated just outside the Power Bldg., by the SW corner. The bridge-type rectifier is situated in the Magnet Control Area of the Power Bldg. and the filter is situated in the basement.

6.10 The a.c. Supply

The name a.c. supply is something of a misnomer. The supply in question does not actually deliver a.c., but delivers d.c. pulses timed to augment the existing 60-cycle oscillation (in the secondary windings of the 60-ton inductor). Just enough energy is supplied each cycle to compensate for the losses that occur.

The a.c. supply consists of a Power Supply No. 1, a charge storage system (also known as "pulse power supply" or "PPS"), and a pulser. These are described in CEA-20, CEA-59, CEA-68, CEA-74, CEA-TM-86, and in the Westinghouse Instruction Manual. Power Supply No. 1 supplies direct current at high potential to the charge storage system. Once each cycle of the synchrotron the pulser delivers current (drawn from the charge storage system) to the primaries of the 60-ton inductor; it does so at approximately the time when the charge on the capacitor banks is a maximum, thus maintaining the 60-cycle oscillation in the magnet powering circuit. A later paragraph discusses the timing of the pulses in greater detail.

Power Supply No. 1 derives its power from a $13.8 \mathrm{kv}$, three-phase, 60-cycle line. Power from the line is transformed by means of an auto-transformer and a rectifier-type transformer to an appropriate voltage and is applied to a three-phase, bridge-type rectifier employing six.ISE-20 ignitrons, firing sequentially. To adjust the output voltage in large steps the operator employs different taps on the rectifier-type transformer; to produce small changes (from $10 \%$ to $100 \%$ of the nominal value provided by the tap) he varies 
the timing of each ignitron. The impedance that Power Supply No. 1 sees is essentially 25 ohms. There is some 360-cycle ripple in the output of Power Supply No. 1, but the filter choke provides ample attenuation.

The charge storage system (or PPS) includes a filter choke, a storage capacitor, and a pulse choke. The filter choke isolates Power Supply No. 1 from the sudden changes that occur in the potential of the storage capacitor; that is, it provides a constant impedance of about $25 \mathrm{ohms}$ for Power Supply No. 1 despite the fact that the capacitor is periodically discharged. The nominal inductance of the filter choke is 0.35 henry, and various taps permit the operator to select values that are $74 \%, 86.5 \%, 93.1 \%, 100 \%$, $114.5 \%$, or $130 \%$ of this. The filter choke is rated at 303 amps rms, 300 amps ave., and 333 amps peak. The maximum voltage rating is $5 \mathrm{kv} \mathrm{rms}$ across the terminals and $10 \mathrm{kv}$ rms to ground. It is air cooled, and is situated in the Power Bldg. basement about $15 \mathrm{ft}$. from the 60-ton inductor.

The storage capacitor has a capacitance of $389.4 \mu \mathrm{f}$ and a voltage rating of $10 \mathrm{kv}$ peak. It consists of 66 individual units connected in parallel. The assembly is mounted in a rack situated in the north portion of the $45 \times 50 \mathrm{ft}$. yard $\mathrm{NE}$ of the Power Bldg. The rack is not grounded. The storage capacitor is being charged throughout about $5 / 6$ of the synchrotron period and is discharged in about $1 / 6$ of that period. During 6-Bev operation of the synchrotron the average potential across the capacitor is about 4000 volts.

The pulse choke smoothes the discharge, spreading it over a period of about 3 milliseconds. The inductance of this choke must, of course, be far less than that of the filter choke, otherwise the discharge would be too prolonged. The nominal inductance of the choke is 2.5 millihenries, i.e., about $1 / 144$ of the inductance of the filter choke. The pulse choke is rated at 303 amps ave., 805 amps rms, and 2700 amps peak. The maximum voltage rating is $5 \mathrm{kv} \mathrm{rms}$ across the terminals and $10 \mathrm{kv} \mathrm{rms}$ to ground. The choke is cooled by air circulated by small fans.

The pulser starts and stops the discharges. The switching is accomplished by a pair of ISE-20 ignitron tubes connected in parallel. A pulse generator tied to the 60cycle line from the Cambridge Electric Light Co. sends a signal to the grids of the ignitrons to fire them, thus starting the discharge. The discharge terminates when the voltage from anode-to-cathode reverses, permitting de-ionization to occur and allowing grid control to be regained.

Because the frequency of delivery of pulses is almost identical to the natural frequency of the magnet powering circuit, the oscillation in that circuit tends to "adjust itself" 
so that the phase (timing) of the pulse approximately coincides with (1) the time the magnet current $i_{\text {mag }}$ has about half the peak value and is decreasing, and (2) the time the voltage across the capacitor bank is a maximum. Circumstance (1) means that the pulse. occurs at a time when there are no orbiting electrons and hence the transient currents do no harm. Circumstance (2). means that the pulses tend to maintain the oscillation, rather than damp it.

The pulser has a rating of 300 amps ave., 800 amps rms, and 2700 amps peak. It is situated in a large cubicle in the Magnet Control Area on the first floor of the Power Bldg.

During 6-Bev operation of the synchrotron, the time-averaged power actually supplied by the pulser is about $1140 \mathrm{kw}$.

\subsection{The Ground Connection}

The magnet power supply is grounded at a point on the output side of the filter of the d.c. supply. The actual point chosen is the midpoint of the series of capacitors on the output side of the filter. Mention should be made also of the high-impedance "grounding" provided by the potentiometric circuit associated with the 24 capacitor banks and described in a previous section.

\subsection{Transmission of Power to the 48 Magnets}

The 24 capacitor banks are connected to the secondary windings of the 60 -ton inductor by 24 pairs of copper bus. bars about $1 \frac{1}{2} " \times 1 / 4^{n}$ in cross section, and the secondary windings are connected to the coils of the 48 magnets by 24 pairs of bare copper tubes that have an outside diameter of $1 \frac{5}{8} "$ and a wall thickness of $1 / 8^{n}$. Each pair serves two magnets connected in series (e.g., Magnets 2 and 3, Magnets 4 and 5, etc.) and thus serves eight series-connected coils. Each quadrant of the ring, i.e., each set of 12 magnets (such as Magnets $2-12$ inclusive) is served by a set of six pairs of tubes that reach the ring via the nearest upstream radial tunnel. In the circular tunnel the tubes rest on brackets attached to the inner wall and are enclosed in a protective housing of metal grillwork. Short horizontal copper bars and flexible vertical drop-out cables connect the tubes to the magnet.coils; the terminals of which are at the back of the magnet. The drop-out cables are enclosed in housings of fiberglass and epoxy resin. 


\subsection{Diode Rectifiers (Not Used)}

The original design of the magnet power supply called for the use of 24 diode rectifiers that would serve to prevent reversal of current in the 24 pairs of magnets and thus minimize difficulties associated with remanence, as explained in CEA-24. Such rectifiers were installed and tried out, but were abandoned in December 1961, for a variety of reasons. One reason was the desire, discussed in a previous section, to allow a slight reversal of current. Another reason was that the transient currents produced by the diodes (at about the moment of injection) proved to be more harmful than had been anticipated.

\subsection{Current Relationships and Energy Storage}

Let the instantaneous amounts of stored energy associated with the coils of a pair of magnets, a pair of secondary windings, and a capacitor bank be denoted by $w_{\text {mag' }}$, $\mathrm{w}_{\text {chk }}$, and $\mathrm{w}_{\text {cap }}$ respectively, and let the corresponding instantaneous currents be called

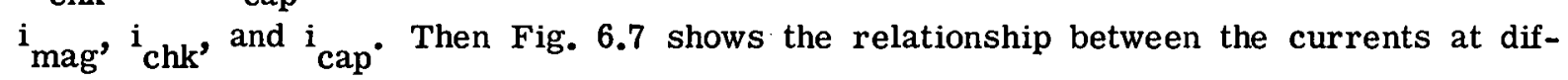
ferent times in the cycle - for the simplified case in which $i_{\text {mag }}$ has exactly a full-biased form, the peak value of $i_{\text {mag }}$ is 761 amps, the inductance $L_{\text {chk }}$ of the pair of secondary windings is $\mathbf{1 3 2 . 8}$ millihenries and is thus just twice that of a pair of magnets, and the capacitor banks are adjusted to produce a natural frequency of 60 cycles/sec. (We assign to each magnet coil and each secondary winding a sensc ouch that the biasing current is deemed positive in each.) Since the biasing current has the same magnitude in the magnets and secondary windings, the average values of $i_{\mathrm{mag}}$ and $i_{\text {chk }}$ are identical, as may be inferred from the figure. Also, if all 24 portions of the circuit are identical and only the desired 60-cycle mode of oscillation occurs, one may regard any pair of magnets as being in parallel with the "next downstream" (or, alternatively, next upstream) secondary windings; accordingly the amplitude of the a.c. component of voltage across the two magnets is identical to that across the pair of secondary windings. However, the phases differ by $\pi$ radians because of the above-mentioned sign convention. Because these voltages are in opposite phase, the corresponding currents $i_{\text {mag }}$ and $i_{\text {chk }}$ likewise are in opposite phase. However, the amplitudes of the a.c. components differ, because the inductances differ; the inductances are in the ratio of 1 to 2 , and consequently the amplitudes are in the inverse ratio, namely 2 to 1 . Taking into account the biasing current, one finds that the peak currents in the magnet coils and the secondary windings are in the ratio of (1) to $\left(\frac{1}{2}+\frac{1}{4}\right)$, 
or 4 to 3. And since the amount of energy stored in an inductance is

$$
\mathrm{W}=\frac{1}{2} \mathrm{Li}^{2}
$$

the peak value of the quantities $W_{\text {mag }}$ and $W_{c h k}$ are in the ratio $(1)\left(4^{2}\right)$ to $(2)\left(3^{2}\right)$ or 8 to 9 , as indicated in Fig. 6.8. Specifically, the values are 458,000 joules and 516,000 joules respectively.

As regards the capacitor system: the instantaneous current $i_{\text {cap }}$ varies symmetrically about zero (see Fig. 6.7) and the instantaneous voltage $e_{\text {cap }}$ varies nearly symmetrically about zero. (Slight asymmetry enters because of the biasing current in the secondary coils; this produces an average potential of (377 amps) $(0.0944 \mathrm{ohms})$ or 36 volts across the individual capacitor bank - a potential that is small compared to the peak voltage of about 10,000 volts.) Accordingly the quantity $W_{\text {cap }}$ has two almost equal peaks each cycle of magnet powering; the value of such a peak is about 7,900 joules. For the entire capacitor bank the value is about 190,000 joules, which is considerably less than the peak value for the set of 48 magnets or for the secondary windings of the 60-ton inductor. The current $i_{\text {cap }}$ equals the difference $\left(i_{\text {mag }}-i_{\text {chk }}\right)$, hence is in phase with the current having greater peak value, namely $i_{\text {mag; }}$ accordingly $e_{\text {cap }}$ lags by $\pi / 2$ radians and $w_{\text {cap }}$ has peak values at $\pi / 2$ and $3 \pi / 2$ radians, as indicated in Fig. 6.8.

During normal 6-Bev operation of the synchrotron the wave form departs from the full-biased form in that a reversal equal to $1.8 \%$ of the biasing current occurs, as explained in a previous section. Also, the d.c. resistance of the coils of a pair of magnets is $0.0472 \mathrm{ohms}$ and the d.c. resistance of a pair of secondary windings is 0.0944 . Other losses occur also. During actual 6-Bev operation the principal currents and energy storage values have the values indicated in Table 6.1.

Table 6.1

Currents and Energy Storage in Magnets and Magnet Power Supply

\begin{tabular}{|c|c|c|c|c|c|}
\hline Component & $\begin{array}{l}\mathrm{i}_{\text {ave }} \\
(\mathrm{amp})\end{array}$ & $\begin{array}{l}i_{\max } \\
(\mathrm{amp})\end{array}$ & $\begin{array}{l}i_{\min } \\
(\mathrm{amp})\end{array}$ & $\begin{array}{l}\text { Weak } \\
\text { (joule) }\end{array}$ & $\begin{array}{l}\text { phase angle } \\
\text { of current } \\
\text { (radian) }\end{array}$ \\
\hline Pair of magnets & 377 & 761 & -7 & $\begin{array}{l}458,000 / 24 \\
\text { or } 19,000\end{array}$ & (0) \\
\hline $\begin{array}{l}\text { Pair of secondary } \\
\text { windings }\end{array}$ & 377 & 569 & 185 & $\begin{array}{l}516,000 / 24 \\
\text { or } 21,500\end{array}$ & $\pi$ \\
\hline Capacitor bank & 0 & 569 & -569 & $\begin{array}{l}190,000 / 24 \\
\text { or } 7,900\end{array}$ & approx. 0 \\
\hline
\end{tabular}


6.15 Magnet Control Area

Special tests and adjustments of the power supply for the 48 magnets are made at, or near, a console in the Magnet Control Area, situated on the ground floor of the Power Building. Routine operation, however, is managed from the Main Control Room.

\subsection{Manufacture}

The individual capacitors were made by Westinghouse Electric and Manufacturing Co., and the racks were built by Robinson Boiler Works Co. Additional installation work was done by Mass. Electric Construction Co.

The core blocks of the 60-ton inductor were made by the Allis-Chalmers Mfg. Co., and the coils were made by National Electric Coil Co. (A full set of spare coils is expected to be available by the spring of 1964.) The inductor was assembled by CEA in 1961 and 1962; assembly was facilitated by a removable octagonal floor section just above the inductor, and by an overhead 10-ton crane. Several of the original coils have failed and have been replaced; replacing a coil takes about eight hours.

Power Supply No. 1, Power Supply No. 2, and the pulser proper were built by Westinghouse.

The filter choke and pulse choke for the a.c. supply system were built by HeviDuty Electric Co.

\subsection{Performance}

The capacitor system has performed well. Few capacitors have failed. The switching system for varying the capacitors has been successful. Variations of capacitance with temperature have been small - probably less than $3 \%$. Variations in potential of the support racks gave some trouble in 1962 and early in 1963. It was found that currents flowed from the racks to ground, and the current varied from one rack to another. Also the potential-to-ground measured at the two ends of a given rack differed appreciably. During rainy weather the troubles became seriously troublesome. The difficulties were largely eliminated by insertion of insulators between racks and the earth. Mylar sheets were used for this purpose starting $2 / 23 / 63$; they may soon be replaced by porcelain insulators.

The 60-ton inductor has performed reasonably well, although difficulties of several types have occurred on many occasions. Procurement of coils having adequate insulation and mechanical strength was a major problem. The original set of coils was made by 
General Electric Co. in 1960; tests made on these coils early in 1961 revealed that the internal insulation was inadequate and allowed short circuits to occur even when the voltages applied were low. Efforts by the manufacturer to correct the difficulty were not successful, and accordingly a set of coils was ordered from National Electric Coil Co. These were installed early in 1962 and performed well for some time. Thereafter several of the coils failed mechanically, for reasons not fully understood, and had to be replaced. By 3/1/64 the construction by National Electric Coil Co. of a new set of coils of improved design was well underway and indeed one of the new coils had been delivered to CEA and installed on the inductor.

Another difficulty was the tendency for the stainless steel end plates of the core blocks to overheat when the inductor was powered for operation levels exceeding 2 or $3 \mathrm{Bev}$. In a first attempt to overcome this tendency, CEA engineers arranged with Bethlehem Steel Co. to mili slots in the end plates, for the purpose of reducing eddy currents; also, water cooled pads were added. The improvement was found to be insufficient, and in June and July 1962 these plates were removed and replaced by water-cooled plates of copper. Because these plates have appreciable overhang, partly bridging the $2 \frac{1}{2}$-inch gap between core blocks and thus helping keep the lines of magnetic flux parallel to the plates, and because copper has a much greater thermal conductivity than stainless steel has, the now plates do not become overheated even during steady operation at 6 Bev level.

The clamps and pressure pads for keeping the coils in place were improved repeatedly and are now perhaps satisfactory; the present clamps are sufficiently compliant to allow the coils to vibrate without undue restraint. Several of the radial tie rods broke and were replaced; late in 1963 a set of rods of improved design was installed. Söme of the vertical tie rods failed also; an improved set was installed in February 1964.

Originally the core blocks were cooled by circulation of silicone oil in vertical annular passages. Leakage of oil was a continual problem - solved late in 1963 by changing to air cooling.

References:

\section{CAP Reports}

CAP-12 Economy of d.c. bias with optimum choke design. E. M. Purcell, 9/23/55. CAP-12a Choke design for symmetrical biased magnet supply. E. M. Purcell, 10/1/55. 


\section{CEA Reports}

CEA-16 Magnet power supply analysis. M. S. Livingston, 11/9/56.

CEA-20 Variable frequency magnet power supply. M. S. Livingston, 1/21/57.

CEA-24 Use of rectifier in magnet power supply. M. S. Livingston and K. W. Robinson, $3 / 13 / 57$.

CEA-43 Analysis of spurious modes in magnet power supply. J. Dekleva and K. W. Robinson, $1 / 3 / 58$.

CEA-59 Magnet power supply. L. A. Young, 6/20/58.

CEA-66(2) Cooling of the ring choke. D. D. Jacobus, 11/1/58.

CEA-68 Operation of the magnet pulse power supply. H. W. Woodsen and L. A. Young, $12 / 1 / 58$.

CEA-74 Dynamic operation of the a.c. magnet power supply. H. W. Woodsen, 8/1/59.

CEA-76 Ring choke for the magnet power supply. D. D. Jacobus, 9/15/59.

CEA-TM Reports

CEA-TM-32 Choke design study. M. S. Livingston, 7/3/58.

CEA-TM-39 Cooling of the ring choke. D. D. Jacobus, 11/1/58.

CEA-TM-59 Cooling the steel core blocks of the magnet power supply ring choke. P. H. Burr, 3/17/60.

CEA-TM-86 Magnet power supply. R. Averill, 6/20/62.

\section{Other Documents}

CEA. specification "Resonant capacitor specifications;" July 17, 1958, Revised Aug. 14, 1958.

Electrical Engineering, Feb. 1961: "Choke core blocks for 6-Bev accelerator."

"Westinghouse Instruction Book for Power Supplies 1 and 2 and the Pulser."

\section{Drawings:}

Drawings included in documents mentioned above, especially the CEA specifications cited. Also CEA drawings E-12E-23C (power supply), E-12E-24C (interconnections), D-12E-61B (controls), E-12E-79A (power supply as a whole; schematic).

Westinghouse drawings 523F363 (controls), 523F364 (identification of components), 523F361 (power); 523F362 (excitation). 


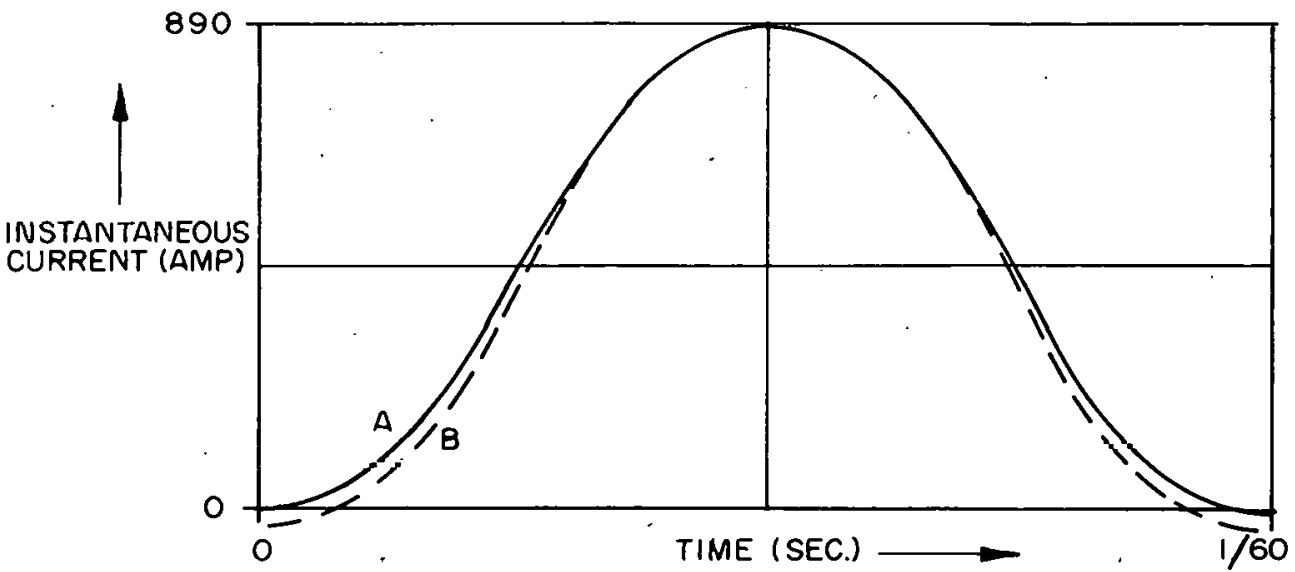

Fig. 6.1. Curve A: ideal, full-biased wave form. Curve B: wave form obtained by reducing the biasing current slightly and increasing the amplitude of the a.c. slightly, in such a manner that the peak current is the same as in $A$ but the amplitude of a.c. exceeds the bias current by $1.8 \%$ of the latter. For clarity of exposition, the difference between the two curves has been exaggerated.

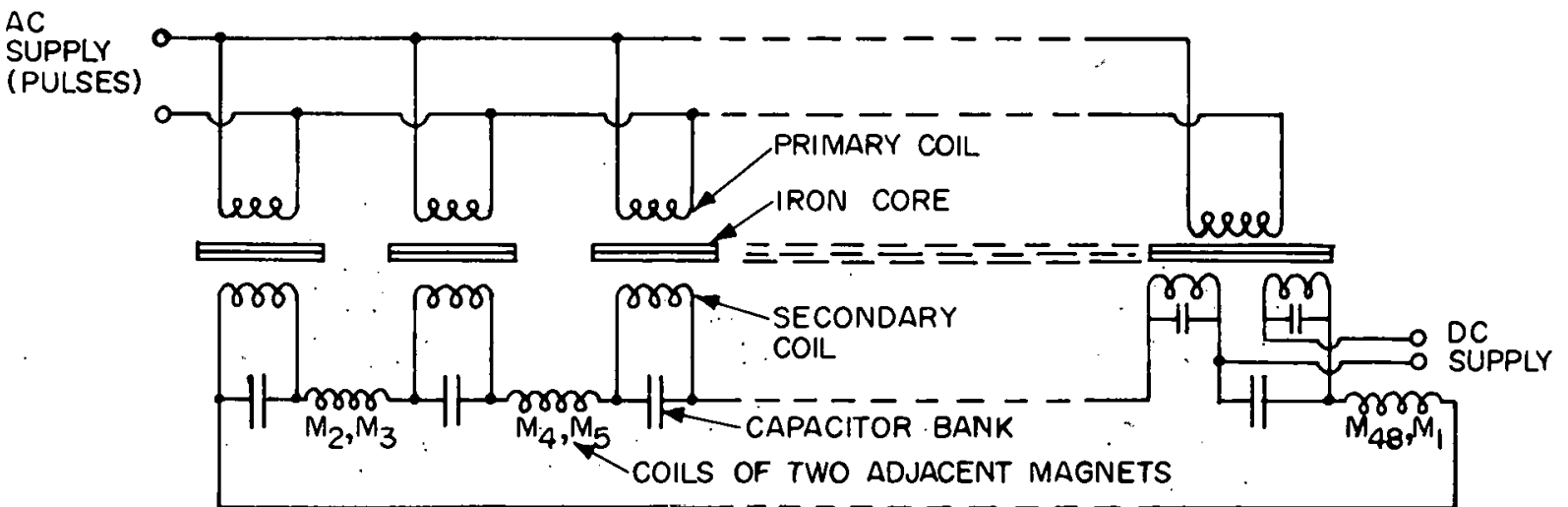

Fig. 6.2. Diagram of circuit for powering the 48 magnets. Only three pairs of magnets are shown. A full diagram would, of course, show 24 pairs。' 


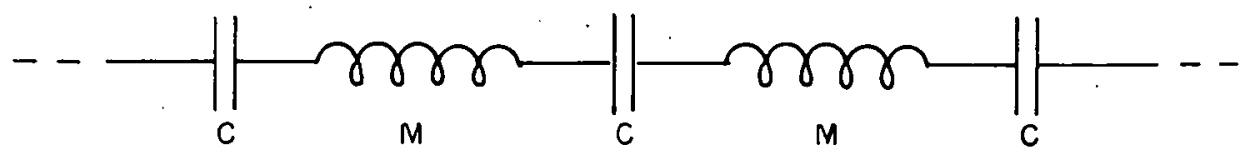

Fig. 6.3. Simplified circuit that can carry a.c. only.

The circuit includes magnet coils (M) and capacitors (C).

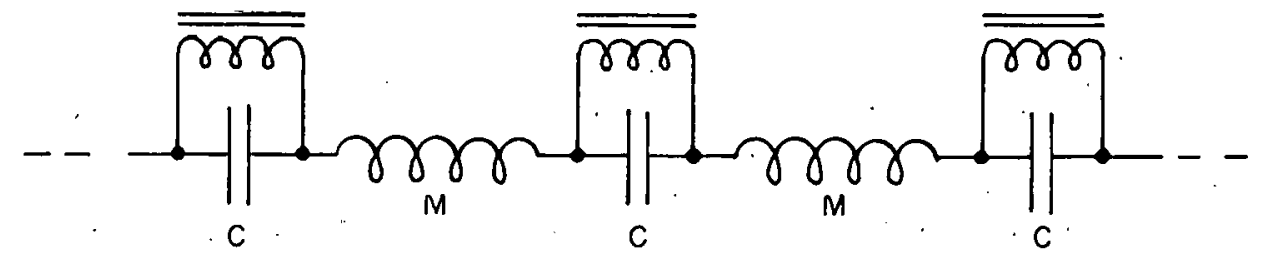

Fig. 6.4. As in previous figure, but with addition of choke-type bypasses to permit.d.c. to flow through the train of magnet coils $M$.

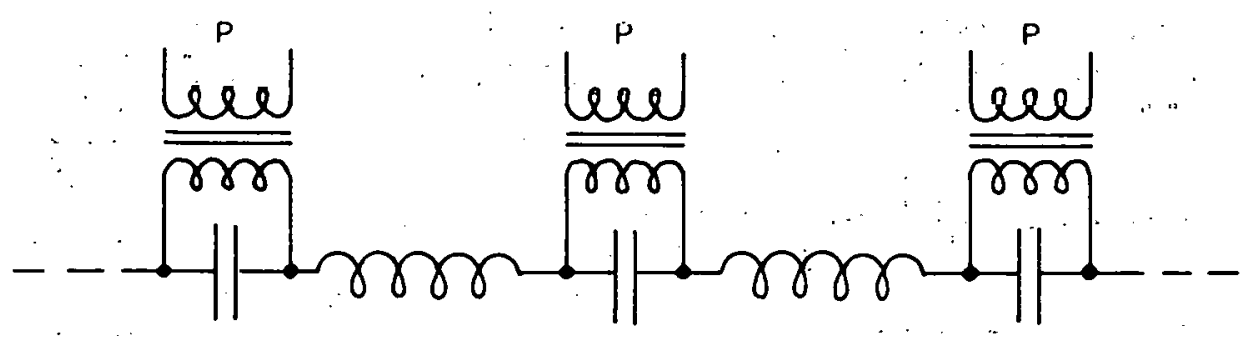

Fig. 6.5. As in previous figure, but with the addition of primary coils $P$ to permit application of pulses of current for maintaining the oscillations. 

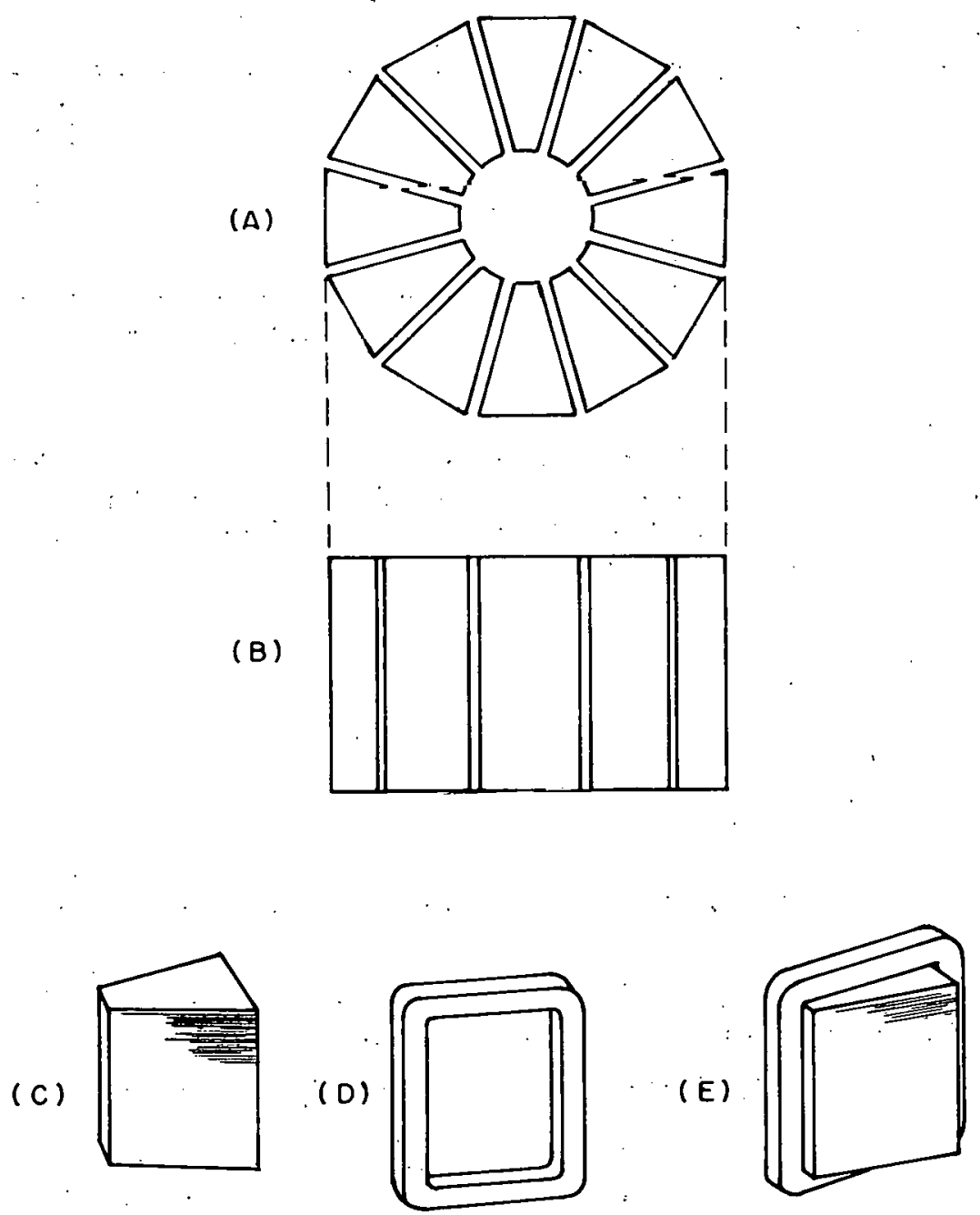

Fig. 6.6. The 60-ton inductor. (a) plan view of set of twelve coreblocks and "air gaps" filled with plates of Micarta; (b) side elevation of same; (c) perspective view of one core block; (d) perspective view of one coil; (e) coil mounted on core block. Not drawn to scale. 


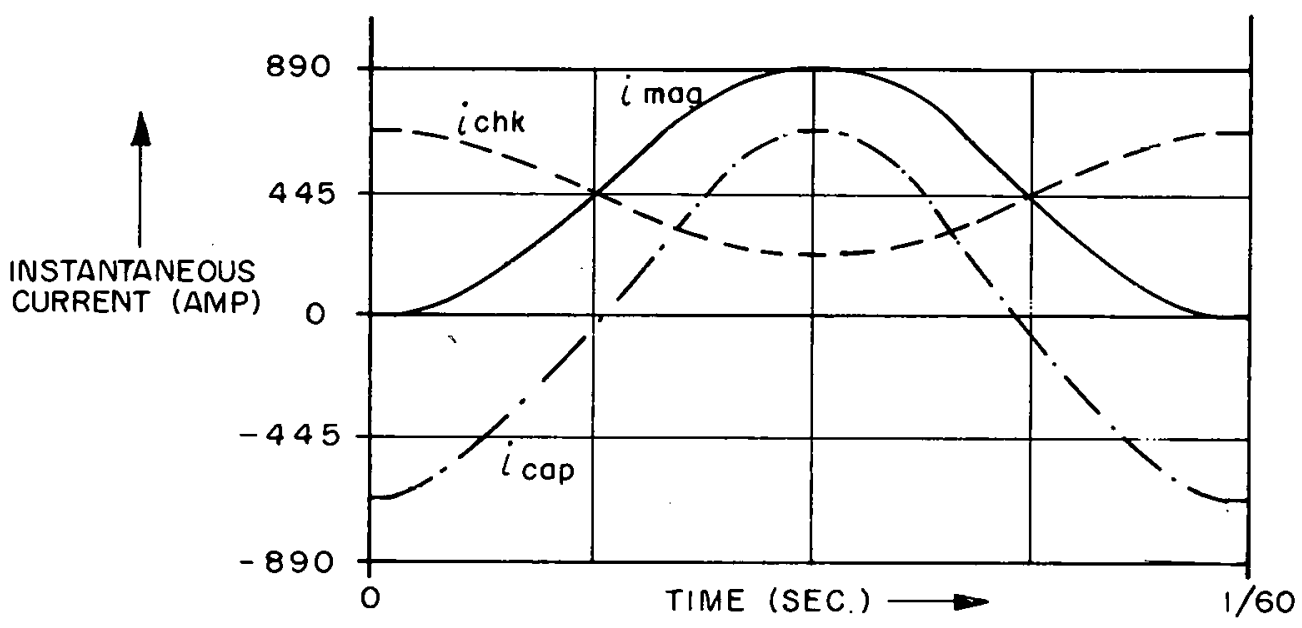

Fig. 6.7. Instantaneous current in the magnet coils, in the secondary windings, and in the capacitor banks, for idealized case of exactly full-biased wave form. Not drawn accurately.

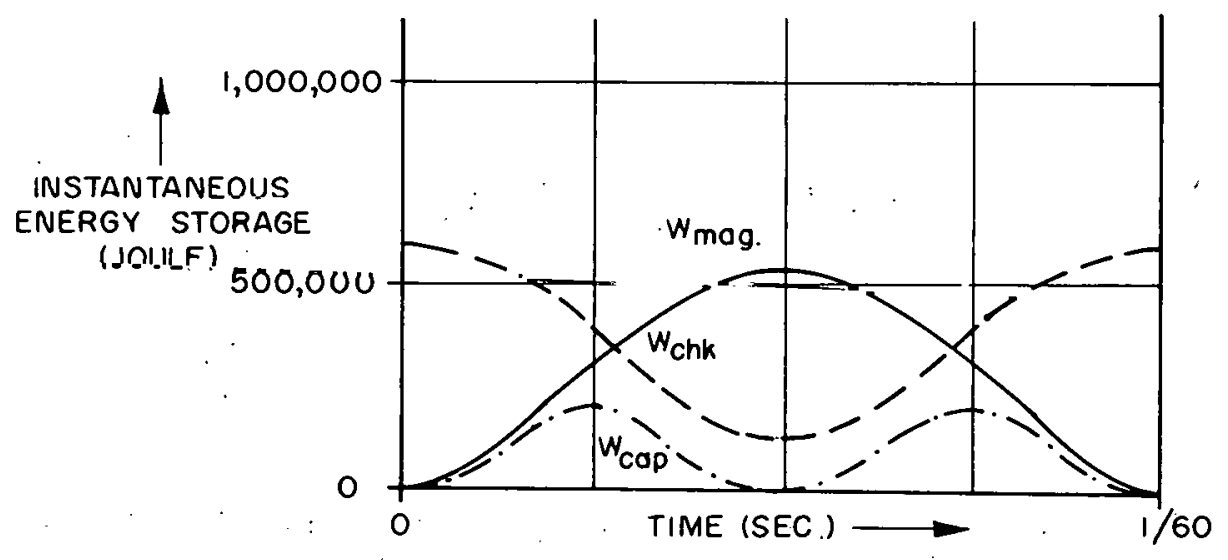

Fig. 6.8. Instantaneous energy storage in the set of 48 magnets, in the 60-ton inductor, and in the set of 24 capacitor banks, for the idealized case of exactly fullbiased wave form. Not drawn accurately. 


\section{Chapter 7}

\section{Pole Face Windings}

$\begin{array}{ll}7.1 & \text { Purpose } \\ 7.2 & \text { Principle } \\ 7.3 & \text { Design } \\ 7.4 & \text { Manufacture } \\ 7.5 & \text { Performance }\end{array}$

\subsection{Purpose}

The purpose of the pole face windings of the 48 magnets is to modify the magnetic field in the magnet gaps at injection, i.e., during the time when electrons are being injected into the orbit and are making their first few turns around the orbit. At injection, the field strength in the gap is small; $\left(\mathrm{B}_{\mathrm{z}}\right)_{0}$, the vertical component of the field at the isomagnetic line, is about 35 gauss. However, the peak field is several kilogauss. Consequently the remanent field is large and, indeed, amounts to a significant fraction of the 35 gauss field, and therefore produces - at injection - significant changes in the field and in the extent to which the field varies with radial position across the gap. In other words, remanence alters the magnet's dipole strength, quadrupole strength, sextupole strength, etc. Remanence is particularly harmful near the ends of the 60-inch-long half-magnets, i.e., near the fringing fields.

The most important consequence of remanence at the time of injection is the change in quadrupole strength, i.e., gradient of field. Calculations made in 1960 by T. L. Collins indicated that the gradient at injection might be off by perhaps $1 / 4$ to $1 / 2$ gauss per inch, and consequently the radial and vertical betatron frequencies $\nu_{x}$ and $\nu_{z}$ at injection might be off by as much as 0.2 or 0.3 units. During operation of the accelerator in 1962 and 1963 it was found that, when the magnets were powered for 1 or $2 \mathrm{Bev}$ energy, $\nu_{x}$ at injection was often as large as 6.5 or 6.6 when the pole face windings were not in use. The value 6.5 implies a half-integral stop-band, hence would cause radial blow-up of the beam immediately following injection. Values above 6.5 at injection cannot be tolerated either, since the high-field value of $\nu_{x}$ is about 6.4 and consequently at some intermediate moment in the acceleration interval the value 6.5 would pertain and beam blow-up would occur. 
The pole face windings are designed to restore the relationship between dipole, quadrupole, and sextupole strengths (at injection) to the proper relationship, i.e., one such that $\nu_{\mathrm{x}}$ and $\nu_{\mathrm{z}}$ will be approximately 6.4 - comparable to the values that pertain at high field - and consequently the amplitudes of betatron oscillations will decrease, not increase, during the carly portion of the acceleration interval.

\subsection{Principle}

A pole face winding consists of an array of copper wires mounted very close to the pole of a magnet and running parallel to the isomagnetic line. The wires carry a small amount of d.c. and hence produce a weak, steady, contribution to the magnetic field. The contribution is significant at injection, i.e., when the magnet field strength is low, and thus can serve as a correction to the field of the magnet. At times when the magnet field is very intense, the contribution by the pole face winding is negligible and it does not matter whether the winding is powered or non-powered.

The wires comprising a dipole winding are connected so as to form a coil that encloses the entire pole, hence produces a contribution that has the same sign over the whole area of the pole.

The wires comprising a quadrupole winding form several sets of loops. Some of the loops are situated near the front part of the pole and carry current in one sense, e.g., clockwise; others are situated near the rear part and carry current in the opposite sense. Thus the field is strengthened in one region, weakened in the other; in other words the gradient (the quadrupole moment) is altered.

The wires comprising the sextupole winding are connected to form many different loops, which are situated and powered so as to alter the gradient of the gradient, i.e., the sextupole moment of the magnet.

Fig. 7.1 indicates the general layouts of the three kinds of windings.

\subsection{Design}

Pole face winding assemblies (abbreviated PFWA's) have been provided for each of the 48 magnets. Since each magnet consists of two separate groups of core blocks and each group has an upper jaw and a lower jaw, a total of 192 PFWA's are employed.

The design of the individual PFWA is indicated in detail in CEA specifications of 8/12/63, which include Drawings S-16M-93C, C-16M-113B, and also Drawings D-16M-115 through D-16M-118 inclusive. Only a brief summary is presented below. 
Each PFWA includes four sets of windings: (a) one-turn dipole winding, (b) twoturn dipole winding, (c) eight-turn quadrupole winding; (d) four-turn sextupole winding. All four are incorporated in a single mechanical assembly, consisting mainly of fiberglass cloth and epoxy resin. Fig. 7.2 indicates the appearance of the assembly and Fig. 7.3 shows the cross section.

The one-turn dipole winding consists of two long straight pieces of copper wire that are embedded in the two long beads (closed-edge bead and open-edge bead). of the assemhly, and includoe ond connection segments diso. The total resistance is about $0.2 \mathrm{ohm}$, and a current of $1 \mathrm{amp}$. can be carried without undue temperature rise. The leads are joined to an Amphenol female connector \#57-40140. The assembly can withstand 700 volts d.c., and is resistant to high temperature, ozone, and radiation.

The two-turn dipole winding is very similar. It can be used if a larger dipole correction is required. If a still larger correction is needed, the one-turn and two-turn windings can be used simultaneously.

The quadrupole winding, comprising 8 turns, includes eight wire segments that run along the crown of the assembly and eight return wires that are situated within the beads; three of the latter wires are in the closed-edge bead and five are in the open-edge bead - an arrangement that minimizes the localized induced voltages and also the overall induced voltage, which otherwise might be so large that breakdown in the insulation might occur or difficulty in designing suitable power supplies might arise. The leads terminate in the same connector mentioned above. The total resistance is about $1.5 \mathrm{ohms}$. The optimum locations of the wires in the crown were determined by means of calculations; see T. L. Collins' informal, one-page memorandum of $7 / 1 / 60$. The calculations indicated that the windings would provide a reasonably uniform gradient-correction throughout a region (in the magnetic median plane) extending about 2 inches to either side of the isomagnetic line; outside of this region the correction would fall off sharply. Slight changes were later made in the specifications of the positions of the wires - to avoid interference with certain sextupole-winding wires. The positions finally adopted are indicated in the specifications and drawings mentioned above; see especially Drawing S-16M-93C.

The sextupole winding includes eight wires, all running along the crown of the assembly. Here too the optimum positions were calculated and the positions finally chosen are shown in Drawing S-16M-93C. The total resistance is about $0.75 \mathrm{ohm}$. A later paragraph describes a scheme for compensating for the high induced voltages that occur in these windings. 
The fiberglass cloth and epoxy resin assembly is shaped to fit a "compromisen pole face profile, indicated in Drawing C-16M-113B. Thus any PFWA can be fitted on any closed magnet, and can also be fitted on any open magnet. However, two types of assemblies, differing only in the "handedness" of the location of the connector, are employed. The types are called "U" and "L"; 96 of each are employed.

So little space was available between the pole faces and the vacuum chamber that it was necessary to specify that the thickness of the crown of the PFWA should not exceed 40 or 50 mils.

Compression springs of beryllium copper, seated in grooves machined in the beads of the PFWA's, urge the opposing PFWA's away from one another, and thus hold each firmly in place on the pole facc. See Fig. 7.4.

By 12/1/63 no arrangement for powering the dipole windings had been completed, and the windings had never been put to any substantial use. In 1961 the expectation was that these windings would be powered by the combination of a backleg winding on the magnet and a large capacitor, which would supply the dipole winding with a 60 -cycle current $180^{\circ}$ out of phase with the magnet field strength. Each magnet would be treated separately; the current in its dipole windings could be adjusted individually, in the circular tunnel. It should be noted, however, that the current provided by this means would vary linearly with the value of peak magnetic field, a relationship that is presumably not ideal.

The quadrupole windings are powered by d.c. supplies situated in the Control Room. All the closed magnet's quadrupole windings are powered as a group, i.e., identically, and all the open magnet's quadrupole windings are powered as a group. The currents supplied to the two groups can be adjusted independently.

The sextupole windings also are powered by direct current from the Control Koom. The current in the windings installed on closed magnets may be adjusted independently of the current in the windings of the open magnets. Whenever the sextupole windings are used, some of the dipole windings are connected to them so as to counteract, or compensate, a large fraction of the induced voltages in the sextupole windings. Otherwise, these latter induced voltages (added up for all closed magnets, or all open magnets) would exceed 1000 volts when the magnets are powered for 6-Bev operation; this would rule out the use of a simple, low-impedance power supply for powering the sextupole windings, and also might cause electrical breakdown of the insulation. When an appropriate number of dipole windings (with appropriate polarity) are connected to the sextupole windings, the 
build-up of a large induced voltage is avoided. Some current then flows in the dipole windings, but it produces only negligible changes in field.

\subsection{Manufacture}

The original set of PFWA's was manufactured by Atkins \& Merrill, Inc., in the period April-October 1961. A total of 105 " $\mathrm{L}^{\prime}$ units and 105 " $\mathrm{U}$ " units were made. Thus there were 9 spare units of each type. In January 1964, 25 additional units of each type were received.

\subsection{Performance}

By $12 / 1 / 63$ the dipole windings had not yet been put into routine use. Consequently no comment on their performance will be included here.

It has been found that, as expected, the quadrupole windings have a large and important effect on $\nu_{x}$ and $\nu_{z}$, the radial and vertical betatron oscillation frequencies. The quadrupole windings on closed magnets produce a large effect on $\nu_{x}$ and a much smaller effect (about $1 / 4$ as great) on $\nu_{z}$; if the windings carry a current of $40 \mathrm{ma}$ in "direct direction" (i.e., direction such that the gradient is increased), $\nu_{x}$ is increased by about 0.1 unit and $\nu_{z}$ is decreased by about 0.025 unit. The quadrupole windings on open magnets produce a converse effect: they have a large effect on $\nu_{z}$ and an effect only about $1 / 4$ as great on $i_{x}$; if the windings carry a current of $40 \mathrm{ma}$ in "direct direction," i.e., so as to increase the gradient, $\nu_{\mathrm{z}}$ is increased by about 0.1 unit and $\nu_{\mathrm{X}}$ is decreased by about 0.025 unit.

The sextupole windings have been used, but not extensively. No comments on their performance will be included here.

For an account of the way in which the pole face windings are used, and a statement of the typical currents employed, see Chap.'27 on ture-up and early operation.

Main References

See specifications, drawings, etc., mentioned above. Additional References

J. Rees "Pole face windings," CEA-35, 6/20/57.

W. A. Shurcliff "Pole face windings: purpose, possible layout, and means of solving the induced voltage problem," 10/25/60 (9 pp.). 

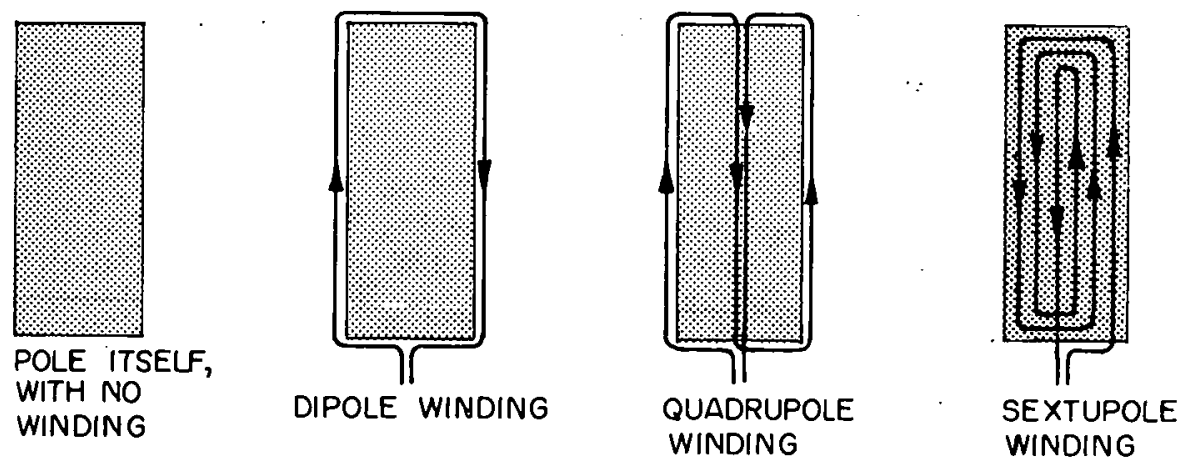

Fig. 7.1. Plan view of the pole of a magnet, with (a) no winding, (b) a dipole winding, (c) quadrupole windings, and (d) sextupole windings. The actual shape of the pole is, of course, much longer and thinner. The arrangement of the wires shown here is schematic, and greatly simplified.

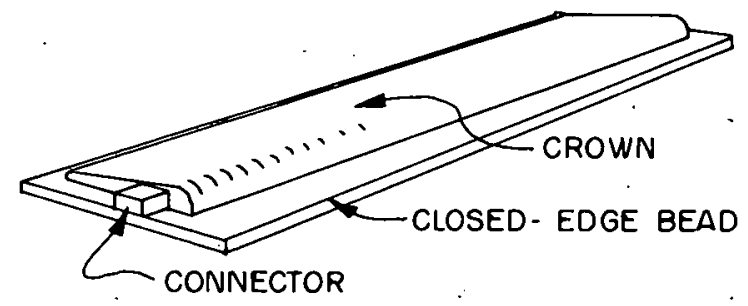

Fig. 7.2. Perspective view of a typical pole face winding assembly.

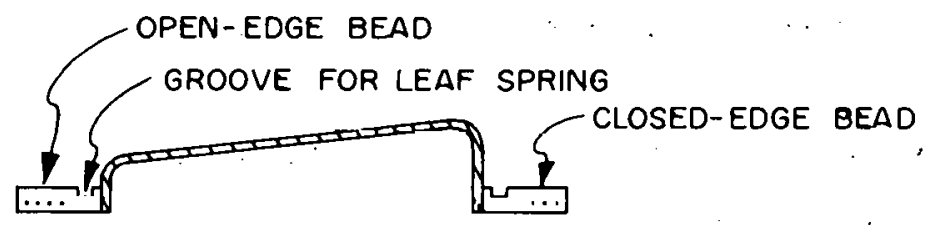

Fig. 7.3. Cross-section (schematic) of a pole face winding assembly. Each dot indicates a wire running parallel to the long axis of the assembly.

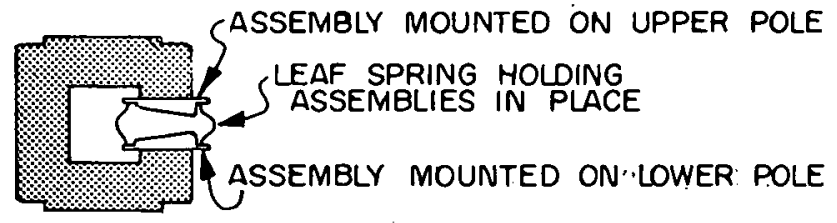

Fig. 7.4. Means for holding the pole face winding assemblies, in: : place on the magnet. 


\section{Chapter 8}

\section{Linac}

8.1 Requir ements

8.2 Design

8.3 Manufacture and installation

8.4 Performance

8.5 Plans for synchronizing linac and synchrotron proper

\subsection{Requirements}

This chapter has been made brief since the expectation is that the present linac will be replaced by one of higher energy within a few years.

The characteristics required of the linac were established by the CEA designers in 1956 and the purchase order was issued in Jan. 1957. During the planning stage the designers gave much attention to the choice of linac output energy. High energy was desired for two main reasons. The first pertains to constancy of orbit radius during the acceleration interval. Since, for many reasons, a constant (or virtually constant) radiofrequency is used in the acceleration system of an electron synchrotron, any increase in speed of electrons-in-orbit results in an increase in radius of the orbit; yet because, in the present accelerator, the width of the useful region of the cross-section of the magnet gap is small, only a small increase in radius - such as 0.4 inch - can be tolerated. This implies a linac output energy of at least $20 \mathrm{Mev}$; an energy of $20 \mathrm{Mev}$ corresponds to an electron speed of $0.9997 \mathrm{c}$ and permits a maximum speed increase of $(1.0000-0.9997) \mathrm{c}$, or $0.03 \%$; the corresponding increase in radius of orbit is approximately 0.4 inch.

A second reason for wanting a high output energy from the linac was the need for reducing various deleterious magnetic effects in the ring of 48 magnets. One such effect was remanent field in the 48 magnets. Measurements made using model magnets showed that the remanent fields in the magnet gaps might amount to about 7 gauss, as compared to the field strength of about 26 gauss that would be required at injection if the output energy of the linac were $20 \mathrm{Mev}$. Another harmful effect was the variations in end-fields * of the 48 magnets. Uniformity was especially hard to achieve here because of the special 
end-plates and end-packets required. A third harmful effect was stray fields in straight sections. Examples of stray fields are the earth's field, the field of the nearby Harvard synchrocyclotron, and fields due to the permanent magnets associated with the high-vacuum pumps. Obviously, the higher the output energy of the linac and hence the higher the field strength of the 48 magnets at injection, the smaller the threat posed by these anomalies.

At the time in question (1956) the optimum choice of energy appeared to be $20 \mathrm{Mev}$. Expectations were that the magnetic anomalies would be relatively small and could be compensated adequately. To have specified - at that stage in the development of commercially produced linacs - a linac of much higher energy would have led to an increase in cost that, at that time, could not be justified.

(As explained in a later section, it became clear during preliminary operation of the synchrotron in 1962 that orbit distortions due to magnetic anomalies at injection constituted a more serious problem than had been anticipated. Variations in stray fields in straight sections proved to be larger and more difficult to control than had been expected. Variations in the end-fields of the magnets were difficult to measure and compensate. In order to reduce the resulting orbit distortions at injection, the operators raised the linac output energy to about 25 or $28 \mathrm{Mev}$, at the cost of a considerable decrease in linac beam intensity. By mid-1963 the need for a linac of considerably higher. energy had become apparent and plans for replacing the original linac with one rated at $70 \mathrm{Mev}$ were initiated.)

Energy spread in the linac output beam was another parameter considered carefully. It was apparent that the spread would have to be kept small if a reasonable fraction of the injected electrons were to be captured into stable orbits. A spread of $\pm 1 \%$ in injection energy leads to a radial spread of \pm 0.42 inch among the equilibrium orbits (measured, say, in Straight Section \#1) and in addition gives rise to horizontal betatron oscillations. Excessive amplitudes of such oscillations can cause a loss of beam intensity before the adiabatic damping discussed in Chap. 3 compacts the beam. If the energy spread is to be kept small, the linac power supplies, and likewise the master oscillator, must be made very stable.

It was clear also that the cross section and angular spread of the beam from the linac, i.e., the emittance ellipse, should be kept small. Otherwise the amplitudes of vertical and horizontal betatron oscillations of the orbiting electrons would be large and many electrons would be lost from the beam. Fortunately, commercially available electron linacs had acceptably. small emittance ellipses. 
The designers of the CEA chose a linac frequency of 2855 megacycles/sec in order to take advantage of the technology and equipment developed for S-band radar and in order to benefit from experience gained by Stanford designers of S-band linacs. The S-band frequency was high enough so that the necessary length of the linac could be small, i.e., of the order of 20 or $30 \mathrm{ft}$.

The designers specified that the linac should provide pulses at the rate of 60 per second and that the length of the individual pulse should be at least $0.7 \mu \mathrm{s}$, i.e., great enough to fill one turn of the orbit. It was desired that the linac have an output intensity of many times the intended intensity of the orbiting beam, i.e., many times 21 ma, since a large fraction of the injected electrons would inevitably be lost to the walls during the first few turns.

\subsection{Design}

The CEA linac, designed and built by the Applied Radiation Corporation (ARCO), is of traveling wave type, employing two 10 -ft.-long sections of iris-loaded, $2855 \mathrm{Mc} / \mathrm{sec}$ (S-band) accelerating waveguides in series. The overall length from the cathode housing at the upstream end to the analyzing magnet at the downstream end is $34 \mathrm{ft}$.

Fig. 8.1 shows a diagram of the equipment. Electrons emitted from a cup-shaped, indirectly heated, oxide-coated cathode are attracted toward, and pass through, the nearby modulating anode, a hollow cylindrical device that has an inside diameter of $1 / 2$ inch. (Cathode and modulating anode are collectively called the gun.) The anode is energized with a flat-top, $6 \mathrm{kv}$ pulse at the moment when injection is to occur; the length of the pulse is about $2 \mu$ s, i.e., several times the duration needed to fill one turn of the orbit. Just before the modulating anode is energized, the entire gun is pulsed 130 to $200 \mathrm{kv}$ negative - for a few microseconds. The modulating anode pulse is timed to coincide with the central (flattest) portion of the 130 to $200 \mathrm{kv}$ negative pulse. The timing of the operation as a whole is governed by a trigger generator which derives its signal from a peaking strip discussed in Chap. 23.

The original intent was that the resulting $200 \mathrm{kev}$ electrons would next pass through a pre-buncher, consisting of a resonant cavity tuned to $2855 \mathrm{Mc} / \mathrm{sec}$. This device was designed to bunch the electrons just before they enter the first accelerating waveguide and thus reduce the energy spread in the beam emerging from the linac. The center-to-center distance between bunches would be about $10 \frac{1}{2} \mathrm{~cm}$. at the downstream end of the linac, and 
somewhat less at the upstream end. In trial operation in 1962 the device performed erratically, and in 1963 it was not used.

The electrons, having now an energy of $200 \mathrm{kev}$ and a speed of $0.7 \mathrm{c}$, pass through a focusing lens (magnetic lens) and then enter the first accelerating waveguide. The $2855 \mathrm{Mc} / \mathrm{sec} \mathrm{rf}$ power is introduced at the upper end via an input coupler, travels to the other end and emerges into a water-cooled matching load. The waveguide is loaded (uniformly, not in progressively spaced, or tapered manner) with iris diaphragms, or washers, to reduce the phase speed to approximately the speed of light in vacuum. (If there were no loading the phase speed would greatly exceed the speed of light in vacuum.) The electrons in the waveguide are accelerated by electric fields of the order of 3 megavolts per ft. Enclosing the waveguide there is (1) a water-cooled jacket, (2) a d.c.-powered solenoidal coil which produces an axial magnetic field that overcomes certain defocusing forces and confines the electrons to a slender region close to the axis, and (3) a set of coils for compensating for the field of the earth.

The second waveguide, colinear with the first, is essentially identical to it.

Just downstream from the second waveguide there is a quick-acting valve that can isolate the linac vacuum system from the synchrotron vacuum system and thus protect the delicate cathode from water. vapor when: and if air suddenly and accidentally enters the synchrotron vacuum system. Rise in pressure adjacent to the valve is sensed automatically, and the valve closes automatically within a few milliseconds; it is actuated by compressed air at 80 psig.

Beyond the valve there is a beam-intensity monitor consisting of a toroidal coil on a ferrite core. The resulting signal is amplified and transmitted via coaxial cable to the Linac Control Room and also the Main Control Room. The monitor is effective whether or not the analyzing magnet (discussed in a later paragraph), is in use.

The frequency: of $\mathrm{rf}$ power used in the two accelerating waveguides is established by - means of a $26.4 \mathrm{Mc} / \mathrm{sec}$, frequency-modulated, master oscillator that also -serves the synchrotron rf system. This oscillator is described in Chap.:10.: The $26.4 \mathrm{Mc} / \mathrm{sec}$ frequency is multiplied by 18 in the TTU-1B of the synchrotron $\mathrm{rf}$ system power supply, and a portion of the resulting $476 \mathrm{Mc} / \mathrm{sec} \mathrm{CW}$.power is diverted to a varactor that multiplies the frequency by exactly 6.

The-resulting (frequency-modulated) $2855 \mathrm{Mc} / \mathrm{sec}$ power is -fed to a $7-\mathrm{kw}$ pulser (klystron) which, when triggered, produces very-fast-rise pulses of several microseconds 
duration. The triggering (discussed in Chap. 23) occurs at a time when the frequency fed to the above-mentioned 7-kw pulser is in the neighborhood of $2854.5 \mathrm{Mc} / \mathrm{sec}$; the range is about 2854.4 to 2854.6. The pulser output is fed to two large amplifier tubes (Varian 820-C klystrons) each having a peak power rating of 5 megawatts. Each of these tubes serves one of the accelerating waveguides of the linac, as explained in a previous section. A remotely controlled attenuator and a phase shifter are provided for the second waveguide. If a pre-buncher were used (see previous section), some of the power for the first waveguide would be diverted to the pre-buncher.

At the downstream end of the linac proper there is a magnetic analyzing system that permits the operator to measure energy and energy spread of the emerging electrons. The 340-lb. electromagnet (ARCO type AP0056) remains passive during normal operation of the linac, and the electrons pass straight through. When the magnet is excited by an appropriate amount of d.c., the electrons are deflected by about $30^{\circ}$ into a drift-tube branch and those that have a certain energy pass through a slit (about $1 / 4$ inch wide) into a water-cooled Faraday cup. By varying the magnet excitation in small steps, the operator can cause electrons of successively different energy to pass through the slit and can. construct an energy distribution curve in terms of the observed value of current from the Faraday cup. (The analyzing system may also be used as a "derailer" for preventing the linac output beam from reaching the synchrotron. When the beam is derailed, and when certain other precautions have been taken, Operations Division men could perhaps be permitted to work in fairly nearby, downstream portions of the Circular Tunnel even while the linac is running.)

Well upstream from the linac there is a small Linac Control Room, separated from the linac proper by a 3 -ft.-thick wall of Type $\mathrm{X}$ shielding blocks made of iron-loaded. concrete. A stream of outdoor air is delivered to this room to prevent build-up of any appreciable concentration of ozone. At the downstream end of the linac - just downstream from the analyzing magnet and Faraday cup - there is a 1-ft.-thick shielding wall which considerably reduces radiation levels downstream from that location.

The linac is situated so that its centerline is parallel to, and $0.7^{\prime \prime}$ radially outward from, the centerline of Straight Section Tank \#1. In other words it is perpendicular to the radius vector from the accelerator centerpoint to Apex \#1 of the 96-sided polygon, and intersects that radius vector at a point $(1.143-0.7)$ or about $0.4^{\text {" }}$ radially inward from that apex. Of course, the injected electrons do not travel straight along this path, but are 
deflected to some extent by the magnetic field of Magnet 48, and are then inflected by the magnetic inflector discussed in the next chapter.

For evacuating the linac, three Drivac high-vacuum pumps are used; one is of 300 liters/sec capacity and the others are of 30 liters/sec capacity.

\subsection{Manufacture and Installation}

The linac was ordered from the Applied Radiation Corp. (ARCO) in Jan. 1957, and was received at CEA in December 1959. By 2/29/60 it had been assembled and operated at a temporary location in the Experimental Hall. In Jan. 1961 two waveguides of improved type were installed by the manufacturer; they eliminated serious difficulty from beam blow-up, and produced a longer and more intense pulse. (One of the new waveguides proved to be defective and was replaced by the manufacturer four weeks later.) Final acceptance tests were completed in March 1961.

On $5 / 12 / 61$ the task of dismantling the linac and transferring it to its final location in the Spur Tunnel was begun, and by $8 / 1 / 61$ the equipment was in operation at the new site. Successful injection tests involving the first 16 magnets were made on $10 / 5 / 61$, and injection into the entire ring of 48 magnets was achieved on $3 / 7 / 62$.

\subsection{Performance}

In the acceptance tests of March 1961 the linac was found capable of producing a 250 ma beam of $20 \mathrm{Mev}$ electrons in pulses lasting $2 \mu \mathrm{s}$, when no pre-buncher was used. When the pre-buncher was used, the performance at $20 \mathrm{Mev}$ was slightly better, and even at $30 \mathrm{Mev}$ an $80 \mathrm{ma}$ beam with an energy spread of only about $\pm 1 \%$ could be achieved. During the spring of 1963 the typical output (with no pre-buncher in use) was a 100 to $150 \mathrm{ma}$ beam of 25 to $28 \mathrm{Mev}$ electrons, with an energy spread of $\pm 1 \%$. The semi-axes of the emittance ellipse pertinent to the "best $60 \%$ of the electrons" were of the order of 0.1 inch and 1 milliradian.

The linac has suffered from a number of specific faults, including leakage, failure of the vacuum pumps, failure of the cathode, electrical breakdown in the anode structure, failure of tubes in the power supply, and presence of $3520 \mathrm{Mc} / \mathrm{sec}$ parasitic oscillations. The performance has been impaired also by imperfect stability of the oscillator, ripple in the power supply output voltage, and timing jitter due to various causes.

Numerous improvements were made. Better waveguides were installed as explained above. Better vacuum pumps, a more stable oscillator, and tubes of improved types were 
installed. The performance at $28 \mathrm{Mev}$ has gradually been improved, the pulse length has been increased, and the general stability has been improved.

Nevertheless the need for a wholly new and superior linac is clear. Higher energy is badly needed, and likewise increased stability and reliability. For further details see Chap. 31.

\subsection{Plans for Synchronizing Linac and Synchrotron Proper}

Early in 1964 plans were being made for (1) causing the linac to produce just one bunch (instead of six bunches) of electrons per period of synchrotron $\mathrm{rf}$ power, and (2) adjusting the phase of this bunch in optimum relationship to the phase of $\mathrm{rf}$ power in the $\mathrm{rf}$ cavities. It was expected that these changes would reduce the beam-loading of the rf cavities and increase the number of electrons accepted into stable orbits.

\section{Bibliography}

CEA and CEA-TM Reports: None

Applied Radiation Corp. "Instruction book for the Cambridge Electron Accelerator" (no date). 


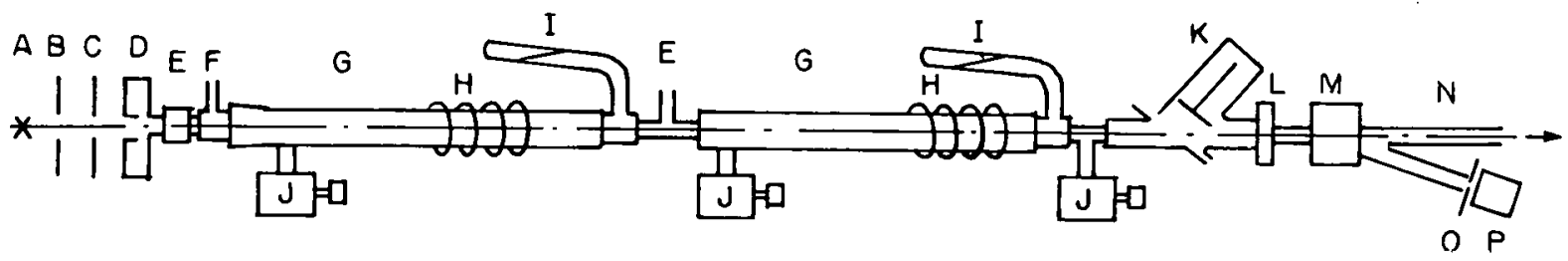

Fig. 8.1. Schematic diagram of main components of the linac proper, including cathode $A$, first anode $B$, main anode $C$, pre-buncher $D$ (not in use at this time, $3 / 1 / 64$ ), focusing lens $E$, radiofrequency power inlet $F$, traveling waveguide $G$, solenoidal coil $H$, matching load $I$, highvacuum pump $J$, quick-acting valve $K$, beam intensity monitor $L$, analyzing magnet $M$, exit tube $N$, analyzing slit $O$, Faraday cup $P$. 


\section{Chapter 9}

\section{Electron Injection}

9.1 Linac beam transport system

9.2 Magnetic inflector

9.3 Electrostatic inflector (superseded)

9.4 Achieving momentum match

\subsection{Linac Beam Transport System}

Between the linac proper and the synchrotron ring there is a 36- $\mathrm{ft}$.-long transport system that conveys the linac emergent beam to the side tube, or spout, on the vacuum chamber in Magnet 48. Fig. 9.1 indicates the more important devices included in the transport system.

At the upstream end, about $3 \mathrm{ft}$. from the linac proper, there is a beam blocker consisting of a $1 \frac{1}{2}$ "-long block of Hevimet that can be lowered into the beam by remote control to insure that no beam can reach the synchrotron. The device can be used as an extra precaution at times when the linac beam is diverted to the Faraday cup and Operations Division men are to be allowed to enter tunnel areas downstream from the linac. At present the beam blocker is used seldom if ever.

The beam then passes a pair of steering coils that may be used to deflect the beam slightly horizontally and vertically and then passes through a housing where, for a short time in 1962, a "Venetian-blind-type attenuator" was situated. (By altering the angle of the vanes of this device, the operator could reduce the intensity of the beam by any desired factor from 0.9 to 0.05 , without having to disturb any of the electrical controls of the linac.) Below the housing a high-vacuum pump is attached.

Just downstream from this housing there is a set of three small quadrupole magnets for focusing the beam horizontally and vertically. They can be used to image the linac-beam cross-over point, which is situated a short distance upstream from the output end of the linac proper, onto the region where the transport system enters the fringing field of Magnet 48. Ordinarily, the currents in these quadrupole magnets are adjusted empirically, i.e., so as to maximize the fraction of linac beam that is captured into stable orbits.

A magnetic chopper, included in the drift tube near the first quadrupole triplet, makes possible the routine elimination of the first and last parts of the beam pulse, so 
that only the most energetically-uniform portion proceeds to the linac. As explained in a later paragraph, only a $0.6 \mu \mathrm{s}$ portion of the pulse serves a useful purpose, and to deliver an unnecessarily long pulse to the synchrotron does harm in several ways, such as (a) increasing the radiation damage to the vacuum chambers, especially Chamber \#1 which the linac beam strikes after the inflector has been de-energized, (b) producing local heating and gassing of this same chamber, (c) contributing slightly to the loading of the rf cavities during the interval preceding the $0.6 \mu \mathrm{s}$ moment of useful injection. Normally the chopper transmits a straight-ahead beam for about $1 \frac{1}{2}$ to $2 \mu \mathrm{s}$, but the length of the interval is variable and may be as short as 0.1 or $0.2 \mu \mathrm{s}$, which may be preferred on some special occasions, for example during initial tune-up of the accelerator. The chopper may be used also to suppress entire pulses and transmit, say, just one pulse out of every 60 .

The chopper consists of two slender air-core magnets, about $5 \mathrm{ft}$. long, that fit inside the drift tube. One of these is energized just before the linac pulse starts, and deflects the first portion of the pulse vertically, so that it strikes a diaphragm situated about $12 \mathrm{ft}$. downstream. This magnet is then de-energized suddenly, allowing the beam to proceed toward the ring. A little later the other magnet is energized suddenly and deflects the final portion of the pulse horizontally so that it too strikes the diaphragm. Charged capacitors provide the high potential for energizing the magnets, and the fast switching. is accomplished by thyratron tubes acting as short-circuiting devices. Each magnet consists merely of two long alumninum rods; the two sets lie in mutually perpendicular planes and hence do not interact. The device was designed and built at CEA and has been in routine use since Jan. 1962.

Just downstream from the chopper there is a set of pick-up coils for measuring the horizontal and vertical transverse displacements of the beam. The output is displayed in the Control Room.

The beam then proceeds toward a stainless steel diaphragm in which there is a 1-inch-square aperture. The diaphragm is 1 inch thick, and is water-cooled.

Next the beam passes through a second quadrupole triplet. In principle this triplet, used in conjunction with the first triplet, can provide exact phase-space match between the linac beam and the synchrotron proper. Actually the second triplet has been used very little; at present it is not used at all. The reason for this is that the first triplet alone appears to provide a sufficient degree of phase-space match.

On emerging from the second triplet, the beam passes successively through a $1.25^{\prime \prime} \times 1.25^{\prime \prime}$ aperture, an intensity monitor, and a transverse displacement monitor. Next 
it passes through a 4 -inch, metal-seat-type gate valve. This is normally open, but is closed manually whenever the synchrotron (but not the linac) is to be let down to air.

Farther downstream there is another transverse displacement monitor and another pair of vertical steering coils. Close to the location where the drift tube joins the spout of Vacuum Chamber 48 there is a horizontal steering coil called the "20-milliradian deflecting coil," which deflects the beam radially outward by whatever amount (in the neighborhood of 20 milliradians) causes it to pass through the optimum region of Straight Section Tank \#1. (If there is a distortion of the equilibrium orbit here, the optimum region may not coincide with the center of the tank.)

After entering Vacuum Chamber 48 the beam passes through the fringing field of the upstream portion of Magnet 48 and passes through the main field region of the downstream portion. Because the strength of the fringing field is hard to measure and varies somewhat with the choice of peak field (and is affected by eddy currents), one cannot calculate the exact path that the electrons will follow.

$i$ The various drift tubes are enclosed in magnetic shields of 20-mil-thick Conetic AA metal.

\section{$9.2 \quad$ Magnetic Inflector}

Finally, the beam reaches the magnetic inflector, situated in Straight Section Tank \#1. Here it is deflected radially outward by about $2 \frac{1}{2}$ degrees, so as to join the intended circular orbit. The operator adjusts the inflection angle empirically so as to maximize the number of electrons that are captured into stable orbits.

The turn-off time of the inflector is crucial, and must not exceed about $0.2 \mu \mathrm{s}$. If it were to exceed $0.7 \mu \mathrm{s}$, which is the time taken by an electron to make one turn around the orbit, every electron that had been inflected into a suitable orbit would pass through the inflector at least once during the turn-off interval and would be deflected out of orbit and would be lost from the beam. If turn-off occurs in $0.15 \mu \mathrm{s}$, only about onefifth of the 750-ft.-long orbiting beam is deflected during turn-off and the other fourfifths remain in orbit. The loss of one fifth is not wholly undesirable, since the resulting 150-ft. gap in the orbiting beam provides a basis for sensing the intensity of the beam and also the transverse displacements, as explained in a later chapter.

As indicated in CEA-TM-57, the inflector is a 60-cm-long, air-core magnet, or coil, consisting of four pairs of conductors symmetrically arranged with respect to the 
centerline of Straight Section 1. Each conductor is a $3 / 8^{n}$ diameter aluminum rod. When the inflector is pulsed with 20 to $25 \mathrm{kv}$, a current of the order of 200 amperes flows in each conductor and a magnetic field of about 60 gauss is produced along the axis of the straight section tank. The turn-off time is approximately $0.15 \mu \mathrm{s}$. The source of high potential is a charged capacitor; the pulse is switched on by means of a thyratron, and is terminated by another thyratron which short-circuits the capacitor.

The inflector was built at CEA and was first used successfully in token injection trials on 10/5/61. However, some shortcomings were apparent and several modifications were made in mid-1962; better insulating bushings were installed, the maximum value of field strength was increased, the stability was improved, eddy currents were reduced, and the high-frequency noise interfering with pick-up coil performance was reduced.

\subsection{Electrostatic Inflector (Superseded)}

In 1959 the synchrotron designers expected that an inflector of electrostatic type would be used. The electrons would be deflected by an electrostatic field between two vertical plates mounted in Straight Section Tank \#1. In 1959 a suitable power supply was ordered from Levinthal Electronic Products (later called Radiation at Stanford). This was received at CEA early in 1961, but was never used.

The plan to use electrostatic inflection was dropped in 1960, when the virtues of magnetic inflection became more evident. The electrostatic system appeared inferior in these respects: (1) Build-up of stray electric charge might occur and might change the extent of inflection erratically. (2) The cross-section of the region of uniform inflecting field would be smaller, which might cause difficulty if an orbit distortion occurred.in this neighborhood and in addition might hamper momentum-matching schemes. (3) To power the device would entail complications.

\subsection{Achieving Momentum Match}

An electron that has a $1 \%$-greater-than-average energy should ideally have a position (at Straight Section \#1) that is 0.43 " farther out radially and a direction that is 2.56 milliradians farther out. If it has such position and direction, a momentum match is achieved, and accordingly the energy deviation does not entail a horizontal betatron oscillation.

Analysis of the momentum matching problem has shown (see CEA-90) that a set of four relatively simple deflecting magnets situated between linac and synchrotron can provide 
a match for all energies within a range of about $1 \%$, and in addition can perform all the functions of the second quadrupole triplet discussed in the previous paragraph. By 11/1/63 a set of four deflecting magnets had been installed (but not tried out). It is hoped that, within a few months, the second quadrupole triplet will be removed and the set of deflecting magnets will be tried out and put to use.

For a discussion of phase-space matching, see CEA-90. Present indications are that empirical adjustment of the first quadrupole triplet of the linac beam transport system provides a sufficient degree of phase-space match.

\section{Bibliography}

G. A. Voss "Magnetic Inflector," CEA-TM-59 of 1/22/60.

W. I. B. Smith "Injection path, phase space and momentum matching," CEA-90 of 8/31/62. (Note: page 2 of this report lists many articles on momentum matching.) 


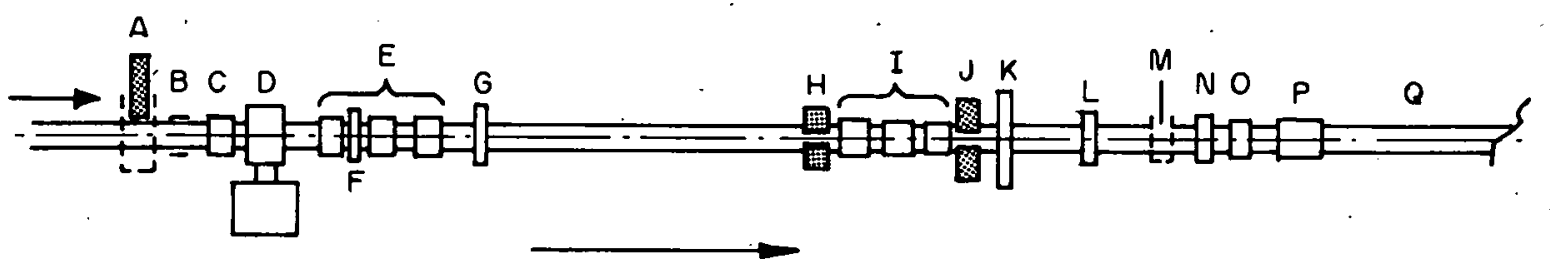

Fig. 9.1. Schematic diagram of devices included in the linac transport system. These include beam blocker $A$, horizontal steering coils B, vertical steering coils C, chamber for Venetian-blind attenuator $D$, first quadrupole triplet $E$, chopper $F$, transverse displacement monitor $G, 1$-inch-square aperture $H$, second quadrupole triplet $I, 1.25$-inch-square aperture $\mathrm{J}$, intensity monitor $\mathrm{K}$, transverse displacement monitor $\mathrm{L}$, gate valve $\mathrm{M}$, transverse displacement monitor $\mathrm{N}$, vertical steering coil $\mathrm{O}, 20$-milliradian horizontal deflecting coil $P$, spout $Q$ of Vacuum Chamber 48. 
$\underline{\text { RF System }}$

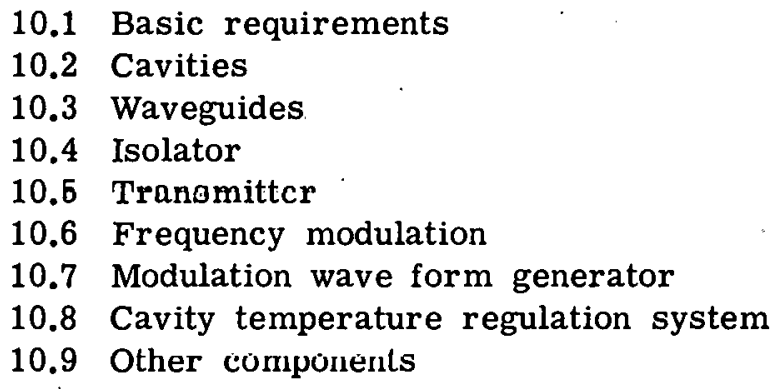

\subsection{Basic Requirements}

Much of the rationale of the accelerator as a whole and the rf system in particular has been indicated in Chap. 3. However, certain crucial aspects of the rf system deserve more detailed discussion; these aspects include: power requirement of the orbiting electron, synchronous phase angle of the accelerator, power required from the rf transmitter, number of $\mathrm{rf}$-cavities, choice of radiofrequency, and transmission of power to the rf cavities. These are discussed in the following paragraphs,

A. Power requirement of the Orbiting Electron. The basic formula for $\Delta \mathrm{E}$, the amount of energy that must be supplied to a highly relativistic electron each time it travels around the synchrotron ring, is as follows:

$$
\Delta E=\frac{4 \pi e^{2}}{3 r}\left(\frac{E}{E_{0}}\right)^{4}+\operatorname{ert} \frac{d B}{d t}
$$

where $e$ is the charge on the electron, $r$ is the radius of curvature of the electron path, $\mathrm{E}$ is the energy of the electron, $\mathrm{E}_{\mathrm{O}}$ is the rest energy of the electron, $T$ is the time taken for the electron to make one turn around the ring, $B$ is the magnetic field strength, and $\mathrm{t}$ is time.

The formula contains two terms. The first indicates the energy needed to make up for the loss by synchrotron radiation. Being proportional to the fourth power of $\mathrm{E}$, the loss becomes enormous when the electron energy is high; at CEA the loss by a 6 . Bev electron is $4.5 \mathrm{Mev}$ per turn. The loss is so great that if the $6 \mathrm{Bev}$ electron received no energy at all it would spiral inward at the rate of about $1 . \mathrm{mm}$ per turn, even if the strength of the magnetic field remained fixed. In no other accelerator in existence is the 
loss as high as this. Such loss usually sets the upper limit on energy achievable in an electron synchrotron. In designing the accelerating system for such a synchrotron, the designer keeps this loss uppermost in his mind.

The second term, involving $\mathrm{dB} / \mathrm{dt}$, is the energy demand - the amount of energy required in order that the momentum of the electron may keep pace with the strengthening guide field, so that the diameter of the path followed will remain practically unchanged. The energy demand is greatest midway in the acceleration interval, owing to the biased sinusoidal wave-form of magnet excitation. The demand is greatest, of course, when the synchrotron is operating at maximum energy (6 Bev); the maximum value is then almost 1 Mev per turn.

B. Synchronous Phase Angle of the Accelerator. As explained in Chap. 3, the synchronous phase angle of the accelerator cannot be set at $90^{\circ}$, but must have some value between $90^{\circ}$ and $180^{\circ}$ if phase stability is to be achieved.

During the latter part of the acceleration interval a phase angle much nearer to $180^{\circ}$ than to $90^{\circ}$ would be desirable to the extent that it provides larger rf buckets and thus permits keeping a larger fraction of the electrons in orbit despite synchrotron oscillations excited by the emission of rather large $(0.1$ to $10 \mathrm{kev})$ quanta of synchrotron radiation. On the other hand a phase angle nearer to $90^{\circ}$ than to $180^{\circ}$ would be desirable to the extent that the very large energy losses by synchrotron radiation can be made up (by energy supplied by the fields in the rf cavities) without the need for using excessively high, hence excessively costly, cavity voltages. It was expected that the optimum value lof synchronous phase angle of the accelerator would be somewhat less than $130^{\circ}$ or $135^{\circ}$.

During the first part of the acceleration interval, i.e., at injection, the advantage of using a phase angle near $180^{\circ}$ is overwhelming. Such a phase angle increases the size of the rf buckets, hence accommodates a greater spread of electron energies and arrival times, and results in increasing the number of electrons captured into stable orbits. Although the cavity voltage must be increased by a large factor to achieve such a phase angle, the voltage remains very small compared to that required at the end of the acceleration interval. Stated differently: the efficiency of use of $\mathrm{rf}$ power at injection is very low, but efficiency is not important at this time.

The parameter controlled by the operator is, of course, the power output of the transmitter. Increasing the output increases the voltages across the accelerating gaps and hence automatically shifts the phase angle toward $180^{\circ}$. 
C. Power Required from the RF Transmitter. The peak value of power required from the rf transmitter occurs near the end of the interval in which the electrons are accelerated to an energy of $6 \mathrm{Bev}$. At that time the transmitter must supply enough power to (1) accelerate the electrons, 1.e., satisfy the energy demand; (2) make up for the power they dissipate via synchrotron radiation, and (3) make up for the losses that occur in the isolator, waveguides, and cavities when the cavity voltages are maintained at the level appropriate to the desired synchronous phase angle.

Early estimates indicated that the power output required of the rf transmitter would be $400 \mathrm{kw}$ peak and $100 \mathrm{kw}$ average. The estimates included an appreciable safety factor. When the rf cavities were built and tested, their efficiency was found to exceed expectations; the losses were less than anticipated, and the actual power requirement was reduced. However, this benefit was partially off set by the addition of the isolator, which dissipates some power and had not been included in the early planning.

Present prospects are that even when the synchrotron is operating at design energy and intensity ( $6 \mathrm{Bev}$ and $10^{11}$ electrons per cycle), the peak power requirement will not exceed $350 \mathrm{kw}$. To date, having a little extra $\mathrm{rf}$ power in hand has proven to be a boon, increasing the simplicity and reliability of operation.

D. Number of $\mathrm{rf}$ Cavities. The designers decided to use $32 \mathrm{rf}$ accelerating chambers, a choice that meant that, during 6-Bev operation of the accelerator, the peak voltage across each chamber would not exceed $6 \mathrm{Mev} / 32$, or about $200 \mathrm{kv}$, assuming a synchronous phase angle of about $135^{\circ}$. One reason for wishing to avoid higher voltage is that the total amount of $\mathrm{rf}$ power required (aside from transmission losses) varies inversely with the. . peak voltage. Another reason is that the danger of electrical breakdown of the cavities is reduced.

In order not to assign one large segment of the accelerator ring to the accelerating chambers and thus spoil the symmetry of the ring, the designers distributed the chambers symmetrically among many straight sections. To avoid preempting too many straight sections, they included two chambers in each of 16 straight sections, as implied by Figs. 10.1 and 10.2. They used chambers that have high $Q$, are tuned very close to the frequency of the rf system, and are tightly coupled - a scheme that provides large shunt resistance. and high efficiency of energy storage.

E. Choice of Radiof requency. Chapter 3 gives the reasons for the choice, in 1957, of a radiofrequency of $475.83 \mathrm{Mc} / \mathrm{sec}$. But the fact is that, in the autumn of 1962 , the 
practice of using a lower radiofrequency, namely $475.72 \mathrm{Mc} / \mathrm{sec}$, was inaugurated. The lower frequency was used in order that the freshly injected electrons, rather than the multi-Bev electrons, would have an equilibrium orbit lying close to the center of the useful region of the magnet-gap cross-section. In any given magnet the freshly injected electrons have a large spread in direction and in transverse position and "more than fill" the useful region of the magnet gap; hence "centering" increases the number captured into stable orbits. The multi-Bev electrons, on the other hand, occupy only a small portion, of the useful region, hence for them centering is less important. The rf cavities were, of course, retuned to be resonant at this $475.72 \mathrm{Mc} / \mathrm{sec}$ frequency.

For a period of time starting in November 1962, occasional use was made of a frequency modulation system intended to reduce beam loading difficulties (see a following section). Just prior to injection, and for about $1 / 2$ to 1 millisecond thereafter, a frequency about 50 to $60 \mathrm{kc} / \mathrm{sec}$ higher than usual was employed, i.e., a frequency of about 475.78 $\mathrm{Mc} / \mathrm{sec}$. The frequency then decreased smoothly to the usual, $475.72 \mathrm{Mc} / \mathrm{sec}$ value.

Starting in January 1964, frequency modulation was used routinely. Several new items of equipment were used, and there was now a new reason for using $\mathrm{fm}$ : the Operations Division was making preparations for achieving exact 6-to-1 synchronism of linac frequency and $\mathrm{rf}$ system frequency (see Chap. 8) and it was necessary to employ, at injection, an $\mathrm{rf}$ system frequency just one-sixth of a frequency within the tuning range of the linac. Six times the $475.72 \mathrm{Mc} / \mathrm{sec}$ frequency was too low for convenient linac operation, but six times a frequency in the neighborhood of 475.75 or $475.78 \mathrm{Mc} / \mathrm{sec}$ was acceptable.

F. Transmission of Power to the rf Cavities. Since the amount of rf power required at the cavities is large, the system for transmitting the power from the rf transmitter must be an efficient one. Also, it must insure synchronism of the rf fields in the 16 cavities to within about $10^{\circ}$ of the phase angle. These requirements are met if the cavities are connected in tightly coupled manner by means of a high-Q, low-loss waveguide ring, this ring itself being connected to the transmitter by a radial link of waveguide. An isolator was included in the radial link to compensate for mismatch and protect the highpower amplifier from reflected surges of power; see Section 10.4. The isolator - which became available only late in the system design - has proved to be one of the most useful and valuable features of the system. It decouples the transmitter's output cavity from the narrow-band accelerating system in a uniquely efficient way, and greatly simplifies operation. 


\subsection{Cavities}

A. Design. Fig. 10.1 shows the longitudinal cross-section of the rf cavity. It shows the two cylindrical chambers (resonators) with openings along the common axis to allow the orbiting electrons to pass through, openings at the top to admit rf power, and openings at the bottom for vacuum pumping. At each axial opening there is a pair of horizontal reentrant members, one above the orbit and one below, which load the cavity capacitatively, serve as drift tubes to reduce the effective transit time of the electrons traversing the cavity, and increase the shunt resistance. (It will be recalled that the shunt resistance of an accelerating structure constitutes a figure of merit.)

The center-to-center distance between the two resonators is $31.53 \mathrm{~cm}$,, which is half the free-space wavelength corresponding to the radiofrequency adopted. The inside diameter is about 16 inches. The slot-shaped, full-width, opening in the dividing wall inductively couples the two resonators to one another, and the 6 "wide slot-shaped openings at the top provide inductive coupling to the adjoining waveguides. See Fig. 10.2.

The individual resonator operates in a "perturbed $\mathrm{TM}_{010}$ mode." (The subscripts indicate the number of nodes with respect to the azimuthal, radial, and longitudinal coordinates.)

Just above each slot-shaped opening at the top there is a $0.3^{n}$-thick disk-shaped window made of high-purity, high-density alumina that absorbs very little of the rf power; made by Western Gold and Platinum Corp., the material is called "Type AL-995, 99.5\% pure aluminum oxide." The window is the barrier separating the evacuated cavity from the unevacuated waveguide. It can withstand a unilateral pressure of 7 atmospheres. The disk is made by a process involving pressing, sintering, and grinding." The edge is chamfered to a conical shape and is provided with brazed-on copper rims to facilitate joining the window to the cavity body by means of a vacuum-tight weld.

In the front side of the cylindrical wall of each chamber there are two recessed sapphire windows. A small probe for measuring the magnitude of the $\mathrm{rf}$ field in the cavity has been inserted in one of the recesses. The windows are also used for viewing the interior of the chamber, to see, for example, whether electrical discharge is occurring there. In the rear wall there is a tuner which consists of a plunger (supported by stainless steel bellows) for varying the resonant frequency of the chamber through a range of about $1.3 \mathrm{Mc} / \mathrm{sec}$. See CEA-TM-75 and CEA-TM-76.

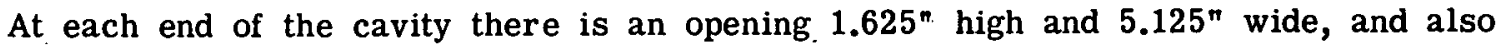
a stainless steel flange (11.000" dia.) to which bellows may be bolted. The flanges serve 
also as fiducial surfaces when the cavity is being installed. Beneath the two 2-inch-wide slot-shaped openings at the base of the cavity there is a stainless steel manifold to which a high-vacuum pump is bolted.

At the downstream end of the cavity, a small pocket has been machined adjacent to the radially outer portion of the opening there in order that a large fraction of the photoelectrons produced there by incident synchrotron radiation may be trapped. If there were no trap, large quantities of photoelectrons might pervade the cavity and cause electrical breakdown.

The resonators are made of oxygen-free, high-conductivity (OFHC) copper. Copper was chosen in order that the cavities might have very high shunt resistance. OFHC copper was required in order to make possible the many hydrogen-atmosphere brazing operations utilized in fabrication, and for no other reason. The walls are thick enough (1.025") so that they do not flex under atmospheric pressure or vibration.

The cooling of the cavities is discussed in a later section.

The $16 \mathrm{rf}$ cavities are situated in Straight Sections 3, 6, 9, etc., i.e., just upstream from Magnets $3,6,9$, etc. The axis of the individual cavity is $1.143^{\text {" }}$ radially inward from the adjacent apex of the 96-sided polygon. See Fig. 13.1. Successive cavities are 554.330" apart on centers.

For further information on the design of cavities, see the reports and specifications listed at the end of this chapter, and also Solar Aircraft Corp. Drawings 006505 and 006510 , and CEA Drawing S-13M-80G.

B. Manufacture. The cavities were made by Solar Aircraft Corp. In making preliminary models the manufacturer encountered difficulties in obtaining vacuum-tightness. To achieve the correct resonant frequency, the manufacturer had to make two prototype . models. Routine production began in Nov. 1960 , the last cavity had been delivered to CEA by $6 / 15 / 61$, and a full set of 16 cavities was in place in the ring by $9 / 15 / 61$.

A total of 18 operable cavities were produced. One is a prototype but, aside from a small variation in height of the base, is fully operable and indeed is in routine use in the ring. Another cavity is used as a beam 'current sensor and is discussed in Chap. 14.

A total of 44-alumina windows were made - by Western Gold and Platinum Corp. The copper rims were brazed on by Ceramaseal, Inc.

C. Performance. The individual cavity performs essentially as expected. The Qvalue of the unloaded cavity is about 25,000 and the shunt resistance is 7.5 megohms. The 
magnitude of coupling between waveguide and cavity is such that if both waveguides joined to a cavity were terminated in matched loads, the loaded $Q$ of the cavity would be about 500. The peak power dissipated per cavity to achieve a peak potential of $200 \mathrm{kv}$ across each chamber (sufficient for $6 \mathrm{Bev}$ operation) is about $12 \mathrm{kw}$. This is somewhat greater than the value calculated from the resistance figure given above, and the excess is accounted for by losses in the waveguide and losses incurred by slight mistuning of the system.

The frequency range (a tunable range of about $1.3 \mathrm{Mc} / \mathrm{sec}$ ) is centered approximately on the desired value; however, manufacturing variations from one cavity to another amount to about half of this range and consequently the available range for the set as a whole is perhaps 0.3 to $0.6 \mathrm{Mc} / \mathrm{sec}$, or about one part in a thousand.

The modal splitting of the cavity, i.e., the difference between the 0-mode and $\pi-$ mode frequencies, amounts to about $2.8 \mathrm{Mc} / \mathrm{sec}$. See CEA-TM-51.

The vacuum integrity has been excellent. Electrical discharge and electrical breakdown within the cavity have occurred occasionally, but seldom if ever while the accelerator is operating routinely. No permanent damage resulted. Early fears that the windows would fail promptly because of heating or because of accumulation of electric charge on the dielectric surfaces have proved groundless. Through $12 / 31 / 63$ only two windows had failed, and these failures were probably due to certain highly abnormal conditions of synchrotron operation.

\subsection{Waveguides}

The waveguide system includes a 750 -ft.-circumference ring situated just above the magnet ring and also a radial link about $1.40 \mathrm{ft}$. long situated in Tunnel 38. The parameters of the waveguide system were chosen so that power of the desired frequency would be transmitted efficiently, well above the cut-off frequency, and the amount of energy stored in the waveguide would be small compared to that stored in the rf cavities, so that the power losses in the waveguide would be relatively small.

The waveguide, of standard WR-1800 type, is made of $1 / 8^{\prime \prime}$ thick $61 \mathrm{ST} 6$ aluminum plates that have been heliarc welded: The cross section of a typical horizontal segment of waveguide is a rectangle 9.000" high and 18.000" wide. The waveguide operates in standard $\mathrm{TE}_{10}$ mode. The electric vector is parallel to the short edge of the rectangle; the magnitude of the vector is great near the center of the cross section and decreases to zero at each side. The nominal attenuation is less than $0.0006 \mathrm{db}$ per linear foot of waveguide. 
The waveguide ring consists of 16 links, each about $45 \mathrm{ft}$. long. Each link extends from the downstream chamber of one cavity to the upstream chamber of the next cavity downstream, as indicated in Fig. 10.2. The 6"'-wide slots between waveguide and cavity are sufficiently large as to provide tight (inductive) coupling.

Each link includes two $11.25^{\circ}$. bends in the horizontal plane so as to conform roughly to the arc of a 118-ft.-radius circle. At each end there is a miter section (" $90^{\circ} \mathrm{E}-$ plane mitered elbow") and a vertical "riser" section that join the main body of the link to the cavities in question. The miter sections are $541.800^{\prime \prime}$ apart on centers. Successive segments of the waveguide link are joined by flanges and $1 / 8^{n}$-thick Parker gaskets.

The design of the waveguide link is such that the two individual resonators coupled by it operate in the same phase $(0$-mode). Since the inverted-U shape of the link has the effect of reversing the electric vector the link must have an electrical length of approximately $\left(n+\frac{1}{2}\right) \lambda$. Here $\lambda$ is the wavelength in the waveguide, rather than in free space, and amounts to about $87 \mathrm{~cm}$., or $140 \%$ of the wavelength in free space. More exactly, the length of a link is $\left(n+\frac{1}{2}+\frac{\delta}{360}\right) \lambda$ where $\delta$ is a small angle chosen so as to minimize excitation of neighboring, unwanted modes of oscillation (especially the so-called waveguide mode) from the desired mode and thus minimize the extents of excitation of the neighboring modes and minimize the power waste associated therewith. Calculations have indicated that $\delta$ should be about $-14.9^{\circ}$.

The electrical length of the waveguide link is adjusted by means of capacitativepost-type phase shifters included near the two ends of the link; see Fig. 10.2. The individual shifter consists of three short rods, each having a rounded end that projects up into the waveguide a short distance. The rods are approximately $\lambda / 4$ apart, hence produce almost no reflection. To change the electrical length of a link, one changes the extents to which the rods intrude into the waveguide. A range of $90^{\circ}$ is provided by the pair of phase shifters.

Although the two individual resonators coupled by a given waveguide link operate in the same phase (0-mode), correspondingly located resonators operate in $\pi$-mode. This applies, for example, to the downstream resonators in Straight Sections 3 and.6. This halfcycle difference is necessary, of course, since the ratio of harmonic order (360) to number of cavities (16) is a half-integer $\left(22 \frac{1}{2}\right)$.

The link includes also a centrally located slotted section that permits insertion of a resonant-post-type short-circuiting device, which may be used to isolate a pertinent 
cavity for tuning and to permit measurement of the standing wave ratio in the waveguide. See CEA-TM-51 and CEA-80. An expansion unit is included also, to allow for thermal expansion and alignment errors; originally a telescoping type of expansion joint was used, but this was replaced in mid-1963 by a bellows, for reasons indicated in a later paragraph. B. Manufacture. The waveguide links were made by I-T-E Circuit Breaker Co., in 1960. All of the regular links were delivered to CEA by $12 / 31 / 60$. The T-link joining the radial run to the waveguide ring was delivered on $6 / 21 / 61$.

C. Performance. In the main, the waveguide system has performed well, with little attention. The Q-value of the typical link slightly exceeds 22,500. Resistive losses in the waveguide are so small that power levels far in excess of those currently used could be accommodated.

The original expansion joints, of telescoping type, caused some troubles. Their lack of angular compliance hampered the alignment of the rf cavities; also the gap between sliding members allowed rf power to leak out, constituting a major source of high-frequency background in the circular tunnel and thus hurting the transmission of pick-up coil signals to the Control Room. Efforts to close the gaps with metallic "fingers" were only partially successful. The bellows-type expansion units are expected to eliminate these problems.

The H-plane switch gave much trouble from arcing and welding, and was removed.

\subsection{Isolator}

Included in the radial waveguide link is a large water-cooled ferrite isolator, which serves two functions. First, it provides instant protection for the transmitter in the event of a surge of power from the waveguide-and-cavity system toward the transmitter. Such a surge occurs when electrical breakdown occurs in a cavity or in the waveguide itself. The isolator blocks the surge by absorbing the great majority of the backward-traveling power. (If surges occurred in many cycles in succession, the isolator might be overheated. This is prevented by the rf-modulation-turn-off device discussed in a later section.)

$\therefore$ The second function is to provide the transmitter with a more perfectly matched load and to decouple the narrow-band accelerating structure from the transmitter output cavity. The isolator does this by absorbing the major part of any power that is reflected back from the cavities or waveguides; also it absorbs power that flows from the cavities as a result of voltages induced there by the bunches comprising the electron beam.

The isolator, a Model 1-UH11 device made specially for this purpose, is about. $5 \mathrm{ft}$. long and $9 \times 18$ inches in cross section. It was delivered to CEA in 1961 and was 
tried out under full load at CEA on $12 / 5 / 61$. The actual value of isolation (attenuation of backward-directed power) is about $13.5 \mathrm{db}$, and the insertion loss (attenuation of forwarddirected power) is $0.35 \mathrm{db}$. Both results are superior to the original minimum specifications, which were $10 \mathrm{db}$ and $0.5 \mathrm{db}$ respectively.

\subsection{Transmitter}

Fig. 10.3 shows the relationship of the $\mathrm{rf}$ transmitter to the $\mathrm{rf}$ power supply as a whole. The basic portion of the transmitter is the RCA TTU-1B, which is an RCA television transmitter employing buffer stages, frequency multipliers, intermediate power amplifier, circulator, video amplifier, modulator, and $1 \mathrm{kw}$ modulation amplifier. The equipment is situated in the RF Control Area of the ground floor of the Power Building.

The TTU-1B is excited by a CEA-built, crystal-controlled, if master oscillator, which serves not only the rf system but also the linac. The device provides automatically cycled frequency modulation between two manually controllable frequencies. It is situated in the Main Control Room. (It is described more fully in the following section.) During tuning tests on rf cavities an RCA oscillator is often used; it provides by a single, manually selected frequency, but has a wider range and is. situated close to the TTU-1B.

Ahead of the video amplifier of the TTU-1B there is a "VSWR protector," provided for the purpose of eliminating the output of the modulator amplifier whenever a sensor situated just downstream from the $\mathrm{rf}$ isolator finds that the amount of backward-reflected power exceeds a specified limit. During the summer of 1963 it was a frequent custom not to use the VSWR protector, but to use, instead; a "crowbar" discussed in a later paragraph.

The sutput of the TTU-1B is fed to a 40-kw driver, which is an RCA amplifier employing a 2041 tetrode.

The output of the driver is fed to the high-power amplifier (HPA). This includes a single tube, an RCA 4612 triode, formerly called a developmental A-15040 superpower triode. By using a single, very powerful tube rather than several less powerful ones, the designers avoided problems involving uniformity of phase and combining power. The 4612 tube is a vertical, cylindrical, double-ended triode that has, at top and bottom, a grid inner cavity and a plate outer cavity. The overall height exceeds $2 \mathrm{ft}$., and the outside diameter is about $1 \mathrm{ft}$. The central region includes a circular array of 96 vertical cathode wires (matrix oxide filaments held taut by springs), an enclosing cylindrical array of grid structures, and, outside this, an enclosing cylindrical anode. The natural frequency of the 
tube's cavities can be altered slightly by means of adjustable shorting bars. The plate potential is very high ( 13 to $15 \mathrm{kv}$ ) and the filament current is very large (about $1500 \mathrm{amps}$ ). This tube, together with the 40-kw driver stage discussed in a later paragraph, is housed in an electrically shielded room on the first floor of the Power Bldg.

During 6-Bev operation of the synchrotron, the average power output of the transmitter is about $50 \mathrm{kw}$ and the peak output is about $300 \mathrm{kw}$. The $\mathrm{rf}$ power issuing from the transmitter travels horizontally, then downward into the basement, then through the isolator and along the radial link in Tunnel 38 to reach the waveguide ring.

The high-voltage power supply is described thus by Musson in "RCA Engineer," $\underline{8}$, 46, 1962:

"The 14-kv plate voltage for the final and driver tubes is supplied from a unitized high-voltage, three-phase, full-wave rectifier utilizing six RCA 857-B mercury vapor tubes. This power supply will provide an output of 20 amperes through an LC filter comprised of a 0.3 -henry reactor (located in the Power Building basement) and a $300 \mu \mu \mathrm{f}$ fused capacitor bank (situated adjacent to the shielded room). It was necessary to provide this relatively large amount of capacitance in the filter to insure an adequate availability of stored energy; this eliminates objectionable voltage drop on the videopulse envelope and avoids objectionable power-line pumping."

The HPA is protected by various devices that sense excessive current, excessive temperature, and drop in rate-of-flow of cooling water. Whenever the plate current becomes moderately excessive, an over-current relay turns off the a.c. input. When the plate current becomes drastically excessive, the capacitor bank is short-circuited in an extremely short time interval by means of an ignitron (fired by a thyratron) acting as a "crowbar."

The transmitter, including the RCA 4612 triode, was made by RCA. It was delivered to CEA on 5/11/61, and was assembled there by RCA engineers by 8/1/61. A second such triode; to serve as a spare, was supplied in $\mathbf{1 9 6 2 .}$

During early trials by RCA engineers, the HPA exhibited various troubles, including parasitic oscillations at 30,1370 , and $2800 \mathrm{Mc} / \mathrm{sec}$, and sporadic generation of gas. On $1 / 27 / 62$ parasitic oscillations caused local overheating in one of the ceramic seals of the 4612 triode and damaged the seal sufficiently so that the tube had to be sent back to the factory for repair. The tuning cavity also caused some trouble. A substitute triode exhibited low gain, and was removed. In other trials, some arcing occurred.

The difficulties were gradually overcome. For example, an improved suppressorloop succeeded in eliminating the principal parasitic oscillations. On about.11/1/61 the 
transmitter met its performance specifications and was accepted by CEA. On 3/2/62 enough $\mathrm{rf}$ power was delivered to the ring to accelerate a token number of electrons to 6 Bev. Actual acceleration took place five days later, on: $3 / 7 / 62$, when the accelerator as a whole operated for the first time. By 12/15/62 RCA engineers had completed the adjusting and testing of the spare triode.

\subsection{Frequency Modulation System}

In November 1962 a CEA-built frequency modulation system was put into use. It shifted the frequency slightly (about 50 or $60 \mathrm{kc} / \mathrm{sec}$ ) immediately prior to injection in order to cause the rf cavities to operate slightly off-resonance, so as to minimize phase shifts due to beam loading by the freshly injected electrons. The shift employed was a shift to higher frequency, since this makes for greater stability of phase of the orbiting electrons. As regards the benefit that resulted from using this $\mathrm{fm}$ system in 1962 and 1963: on occasions when the synchrotron was running well and the beam intensity was limited by beam loading, the use of $\mathrm{fm}$ permitted a significant increase in intensity. Often, however, other factors (not well understood) were dominant, and the use of frequency modulation had little or no effect. Accordingly, in 1963 it was often the practice not to use the fm system.

Starting in January 1964 a somewhat different frequency modulation system was put into use. This was intended not only to reduce beam loading at injection and to approximately center the injected beam in the useful portion of the magnet-gap cross-section, but also to make it feasible to achieve exact 6-to-1 synchronism of frequency of the linac and frequency of the main rf system; see Chap. 8. The heart of the new $\mathrm{fm}$ unit is a crystal-controlled master oscillator, built at CEA and situated in the Control Room, that includes two control knobs, one controlling the (higher) frequency at the start of the acceleration interval and the other controlling the (lower) frequency throughout the latter part of the interval. The respective frequency ranges of the master oscillator are 26.429 to $26.431 \mathrm{Mc} / \mathrm{sec}$ and 26.426 to $26.428 \mathrm{Mc} / \mathrm{sec}$, which, after multiplication by the appropriate multipliers $(6 \times 18$ and $1 \times 18$ respectively), become 2854.33 to $2854.55 \mathrm{Mc} / \mathrm{sec}$ and 475.67 to $475.71 \mathrm{Mc} / \mathrm{sec}$. The centers of the respective ranges are $2854.44 \mathrm{Mc} / \mathrm{sec}$ (i.e., 6 times $475.74 \mathrm{Mc} / \mathrm{sec}$ ) and $475.69 \mathrm{Mc} / \mathrm{sec}$. The two $26 \mathrm{Mc} / \mathrm{sec}$ signals are fed sequentially to the TTU-1B described in the previous section. Of course, the two frequencies "blend into one another" smoothly; after injection the frequency relaxes from the injection value to the steady state value in $1 / 2$ to 1 millisecond. 
The fm system provides an opportunity for compensating automatically for any small temperature change, and hence change in resonant frequency, of the rf cavities. In January 1964 plans were being made for incorporating in the master oscillator a circuit that would cause the steady state frequency to "track" the change in cavily frequency.

\subsection{Modulation Wave Form Generator}

The modulation wave form generator controls the output of the $1 \mathrm{kw}$ amplifier (and hence the transmitter output also) from moment to moment in the 8 millisecond acceleration interval. The wave form is selected by the operator by turning three knobs, called " $\mathrm{C}_{0}$," "B dot," and " $\mathrm{B}^{4}$." $\underline{\mathrm{Knob} \mathrm{C}_{\mathrm{O}}}$ controls the amplitude of the rf power at injection; low power suffices since the magnetic field is increasing only very slowly at this time and the power loss by synchrotron radiation is negligible. Knob B-dot governs the extent to which the amplitude increases with $\dot{\mathrm{B}}$, or $\mathrm{dB} / \mathrm{dt}$; thus it is concerned with the energy demand associated with the rate of increase of field in the 48 magnets. Knob $\mathrm{B}^{4}$ governs the extent to which the amplitude increases with $\mathrm{B}^{4}$; thus it is concerned with losses by synchrotron radiation, which are extremely large just before the end of the acceleration interval. The three knobs operate simultaneously, of course, to provide an amplitude proportional to $\left(\mathrm{C}_{0}+\mathrm{C}_{1} \mathrm{~B}+\mathrm{C}_{2} \mathrm{~B}^{4}\right)$, where $\mathrm{C}_{0}, \mathrm{C}_{1}$, and $\mathrm{C}_{2}$ are constants selected by the operator by means of the knobs. (There is a fourth knob, Knob LLB, which defines a "least lower bound," or "floor" to the amplitude. Ordinarily, the values of $\mathrm{C}_{0}, \mathrm{C}_{1}$, and $\mathrm{C}_{2}$ are such that no floor is needed; thus the fourth knob is left at zero. There is another knob, Knob B that is sometimes used when the accelerator is operating at low energy; it governs the extent to which amplitude increases with B itself, and helps smooth the spillout process.)

The modulation wave form generator was built at CEA and was put into use in September 1962. The value of $\dot{B}$ is sensed by a back-leg winding on one of the 48 magnets. By integrating this signal, one obtains $B$ itself. A signal roughly proportional to $B^{4}$ is obtained with the aid of a triode having an empirically adjusted, fixed, grid potential and a plate potential that is proportional to $B$ itself. The wave form that results is displayed on CRO's in the RF Control Room and Main Control Room.

Back Porch. When the 6-Bev electrons are caused to strike an internal target by means of the rf-turn-off method (see Chap. 16), they spiral inward rapidly (at the rate of . about $1 \mathrm{~mm}$. per turn) and strike the target throughout a time interval of only about $10 \mu \mathrm{s}$. 
A much longer spill time is desired by many experimentalists, and can be provided if the level of $\mathrm{rf}$ power is adjusted to a critical, intermediate (and gradually decreasing) value such that most of the electrons remain in stable orbits but, in each microsecond, a few that have large amplitude of synchrotron oscillation lose synchronism with the rf field, spiral inward, and strike the target. Provision for such a "back porch" power level is included in the modulation wave form generator: the operator can select the height, slope, and duration of the porch. Spill times as long as 500 or $1000 \mu \mathrm{s}$ can be achieved. Usually, the beam-bump method of target engagement is used, and no back porch is needed.

\subsection{Cavity Temperature Regulation System}

The cavity temperature regulation system is called on to perform two functions: heating the cavities before the accelerator is started and cooling them after it has been started. Before the accelerator is started the cavities must be heated to such temperature (about $105^{\circ} \mathrm{F}$ ) that the resonant frequency of the cavities matches the frequency of the $\mathrm{rf}$ transmitter. If the cavity temperature were only $70^{\circ} \mathrm{F}$ the cavity dimensions would be too small and the resonant frequency would be too large by about $150 \mathrm{kc} / \mathrm{sec}$. (The temperature coefficient is $-4.5 \mathrm{kc} / \mathrm{sec}$ per deg. F.) Consequently the power level in the cavities would be very low (assuming that the transmitter frequency remains unchanged) and the orbiting electrons could not be accelerated to high energy. Furthermore the level of power reflected toward the isolator and transmitter would be excessive. The resistive losses in the cavities would be so small that automatic warm-up of the cavities would not occur within an acceptable time.

During normal operation of the synchrotron the standing wave ratio in the cavities is high, the resistive losses are large (about $2 \mathrm{kw}$ per cavity, during 6-Bev operation), and the cavities must be cooled.

The required precision of temperature control is governed, ordinarily, by the level of reflected power that can be tolerated. During 6-Bev operation a precision of the order of $1 / 2 \mathrm{deg} . \mathrm{F}$ is desirable, and during $1-\mathrm{Bev}$ operation a precision of $5^{\circ} \mathrm{F}$ is ample.

The temperature regulation system was designed in broad terms by the CEA staff, and the control system was designed by Minneapolis Honeywell Co. Construction was complete by Nov. 1961. The system supplies water of appropriate temperature to the 16 cavities in parallel, from a common supply made up by combining, in suitable proportions, cool water (from a heat exchanger which in turn is cooled by cooling-tower water) and 
hot water (heated by a steam line). The water employed is "machine water," discussed in Chap. 22. Controls and temperature recorders are situated in the RF Control Area.

The system has performed well. However, the relative level of reflected power in the $\mathrm{rf}$ waveguide system changes occasionally (during 6-Bev operation) by amounts that slightly exceed tolerance and suggest that temperature variations as great as $1^{\circ} \mathrm{F}$ occur. It is usually the custom for an operator to compensate for such variations by empirical change in the rf frequency. In Dec. 1963 plans were being made for a tracking system that would make the necessary changes automatically.

\subsection{Other Components}

A. Cradles. The $\mathrm{rf}$ cavities rest on aluminum cradles which in turn rest on the ends of the adjacent magnet girders. There is a large central hole in the cradle to allow room for the vacuum manifold at the base of the cavity. A high-vacuum pump is bolted to. the base-flange of this manifold. (The prototype cavity has a slight excessive vertical dimension, hence is provided with a cradle that has been specially machined to allow for this height excess.)

B. Dummy Load. During tests, the transmitter is sometimes coupled to a dummy load instead of to the waveguide ring. The dummy load consists of a long waveguide section that contains two slightly-obliquely-mounted slender cones of glass through which flows impure, electrically conductive water (tap water) which absorbs the rf power. When the dummy load is to be used, it is connected to the transmitter by means of a special waveguide link. (Formerly a special H-plane switch was used. This gave trouble from arcing and welding, and was removed.)

$\underline{\text { References }}$

CEA Reports

CEA-11 "Radiof requency acceleration. Part II," (See CAP-25), K. W. Robinson, 9/10/56.

CEA-17 "Radiofrequency parameters," K. W. Robinson, 11/21/56.

CEA-38 "Radiofrequency acceleration. Part III," K. W. Robinson, 7/25/57.

CEA-55 "Introduction of power into the rf system," J. R. Rees, 4/2/58. 
CEA-67 "Resonance frequencies of ring circuits," J. Dekleva and J. R. Rees, 11/25/58.

CEA-80 "Tuning of the $\mathrm{rf}$ accelerating system of the CEA," J. Dekleva and J. R. Rees, 2/16/60.

CEA-TM Reports

CEA-TM-35 "Provisions for tuning the rf ring in place," J. Dekleva and J. R. Rees, 8/15/58.

CEA-TM-51 "Objective electrical specifications for CEA rf cavities," J. Dekleva and J. R. Rees, 10/9/59.

CEA-TM-61 "Cooling the rf cavity," D. D. Jacobus, 5/10/60.

CEA-TM-76 "Electrical properties and performance of rf resonant cavities for CEA," L. C. Robinson, 12/15/61.

\section{Miscellaneous}

J.'R. Rees "Specification for waveguide system," 5/25/59.

CEA Staff "Specification for rf cavities," 6/18/59.

J. R. Rees, L. C: Robinson, A. V. Dubsky "Properties of the rf cavity Prototype I," 4/27/60.

Solar Aircraft Co. "RF cavity for CEA: status report $1, " 5 / 13 / 60$.

K. W. Robinson "Radiofrequency system of the CEA," IRE Transactions on Microwave Theory and Techniques, MTT-8, 593, 1960.

C. H. Musson "High-power rf generator for the CEA," RCA Engineer $8,46,1902$.

RCA "Cambridge Electron Accelerator RF Generator (ES28800)," an undated manual. 


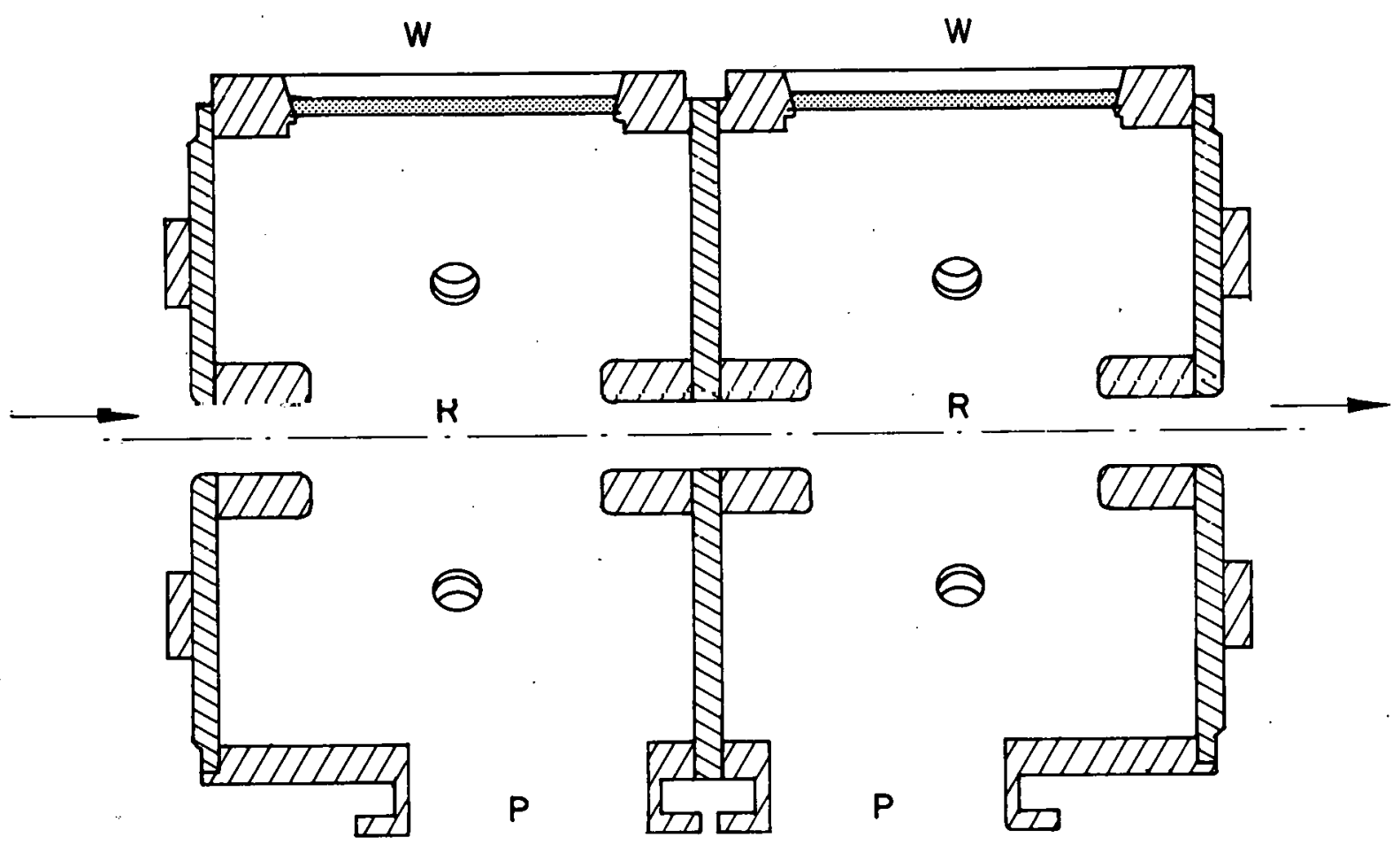

Fig. 10.1. Vertical longitudinal section of an rf cavity, showing the two resonators $R$, the two alumina ceramic windows $W$, the re-entrant surfaces, and ports $P$ leading to the vacuum pump.

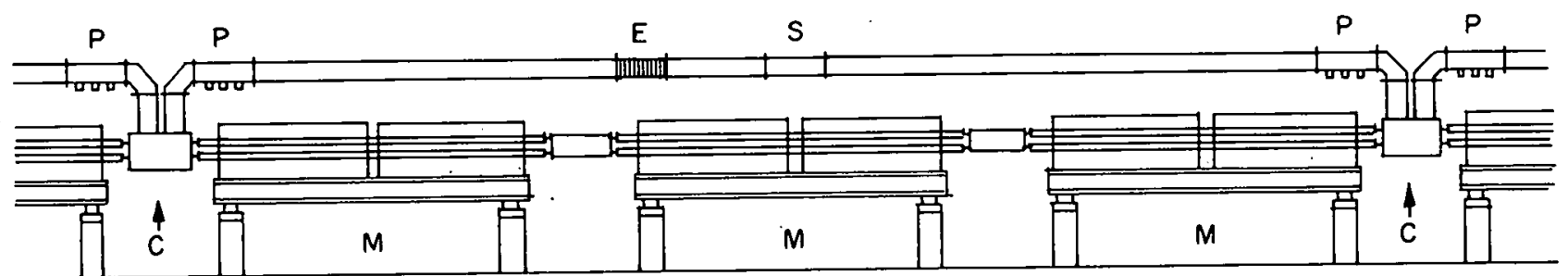

Fig. 10.2. Cavities and waveguide link. Two cavities $\mathrm{C}$ are shown, with three magnets $M$ intervening. Each waveguide link contains two phase shifters $P$, an expansion unit $E$, and a slotted section $S$. 


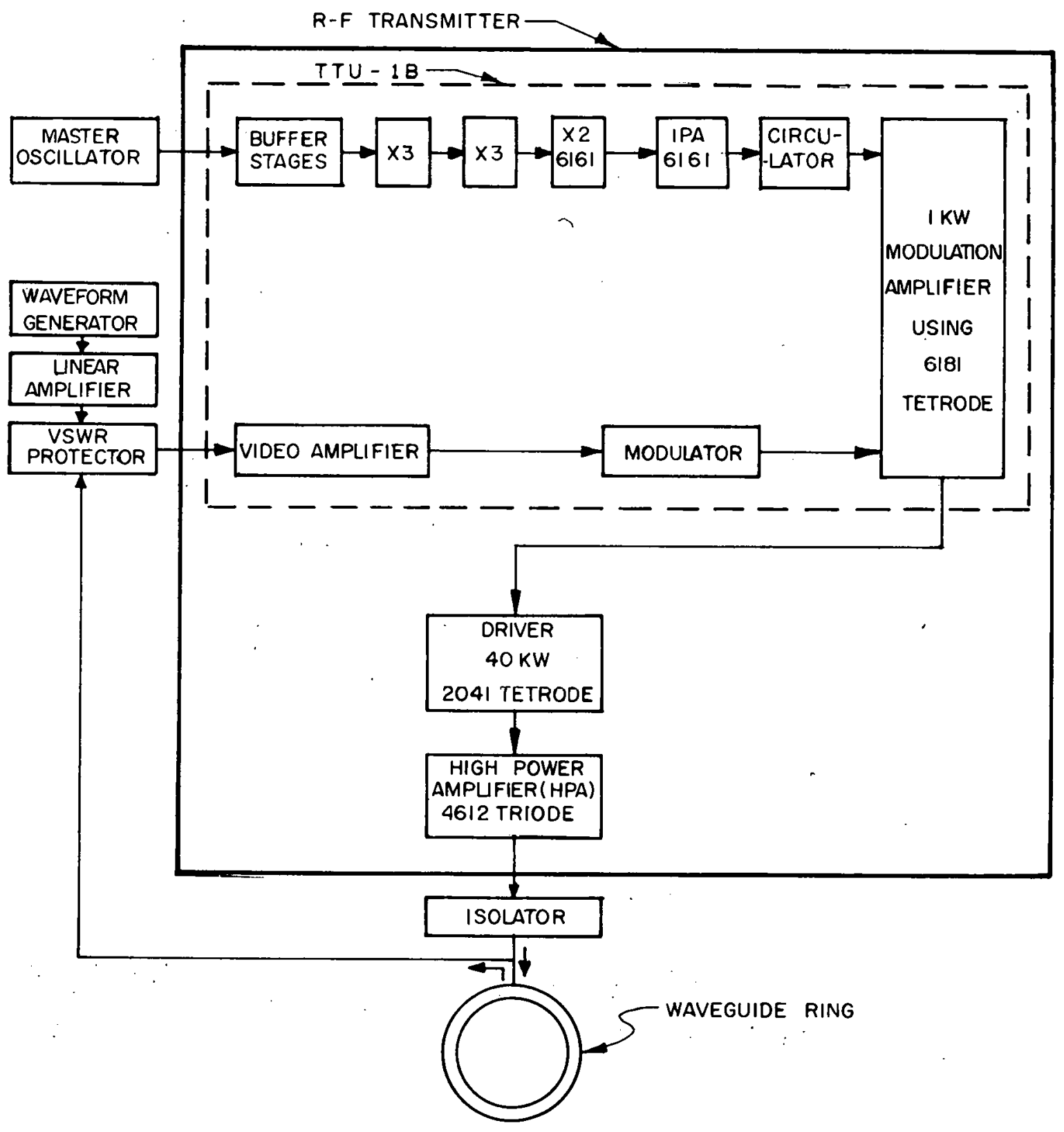

Fig. 10.3. Power supply for rf system. 


\section{Chapter 11}

\section{Vacuum Chambers}

\subsection{Requirements}

11.2 Vacuum chambers of oval ring type

11.3 Vacuum chambers of new types

11.4 Injection chambers

11.5 Exit ohamboro for photon bcama

11.6 Exit chamber for electron beam

11.7. Bellows

11.8 Vacuum seals

\subsection{Requirements}

The 48 vacuum chambers, together with the rf cavities and straight section tanks, make it possible to provide an evacuated path for the orbiting electrons. The vacuum integrity of the chambers must be high enough so that the pressure may be reduced to such an extent that scattering of the orbiting electrons by the residual gas is not appreciable. Most of the orbiting electrons are scattered many times during their 8-millisecond, 1400mile trip within the vacuum ring. The multiple scattering is harmful because it produces small changes in direction of the individual electron and thus increases the amplitude of betatron oscillation. The rms angle $\theta_{\mathrm{rms}}$ through which an electron is deviated (in traversing a region containing a small amount of gas and in being multiply scattered thereby) may be computed from the relation

$$
\theta_{\mathrm{rms}} \cong \frac{(21 \mathrm{Mev})\left(\mathrm{X} / \mathrm{X}_{\mathrm{O}}\right)^{\frac{1}{2}}}{\mathrm{E}}
$$

where $\mathrm{E}$. is the electron energy in Mev, $\mathrm{X}$ is the pathlength in the given region, and $\mathrm{X}_{\mathrm{o}}$ is the "radiation length" in that region. (Pathlength and radiation length are usually expressed in terms of gram $/ \mathrm{cm}^{2}$.) Calculations suggest that if the pressure is kept below $1 \times 10^{-5}$ torr, $\mathrm{X}$ is sufficiently small that no more than a few percent of the electrons are lost by scattering.

Another reason for keeping the pressure below about $10^{-5}$ torr is to make it extremely unlikely that electrical breakdown will occur in the $\mathrm{rf}$ cavities even when very high voltages are used there, as during $6-\mathrm{Bev}$ operation of the synchrotron. A reason for preferring to keep the pressure well below $10^{-5}$ torr is indicated in the following chapter, 
which deals with the high-vacuum pumps and their ideosyncrasies. The pressure requirements are discussed in greater detail in Chap. 12, which also states the pressures actually achieved.

The cross section of the interior of the vacuum chamber must be wide enough to accommodate the large horizontal oscillations that occur immediately following injection. In closed (radially focusing) magnets the horizontal oscillations have greater amplitude than in open magnets, as explained in Chap. 3 ; hence it is appropriate that chambers intended for closed magnets be wider than those for open magnets. However, there is little benefit from having the width exceed 5 inches, since the gradient of the magnetic field deteriorates sharply at 2 to $2 \frac{1}{2}$ inches from the vertical plane that includes the isomagnetic line.

The height of the cross section can be less, since synchrotron oscillations produce excursions that are horizontal, not vertical. The height must be great enough, however, to satisfy two requirements. First, reasonably large amplitudes of vertical betatron oscillations must be accommodated; they are large at injection and, in general, are larger in open (vertically focusing) magnets than in closed magnets. Second, the cross-sectional area of the chamber must be great enough to afford pneumatic conductance such that the pressure can be kept below $10^{-5}$ torr even at the center of the chamber, i.e., about eight feet from the nearest vacuum pump. The conductance should be of the order of 10 liters/sec at a pressure of $2 \times 10^{-6}$ torr.

The height must, of course, be small enough so that the chamber can be fitted between the poles of the magnet and still leave room for the pole-face-winding assembly. This is an important limitation on the chambers for closed magnets, since these chambers (necessarily introduced from the front of the magnet) must be thin enough to pass through the narrowest part of the gap, where the height available between pole-face-winding assemblies is only about $1.480 "$.

The chamber must be built of non-magnetic material, if large magnetic forces and severe vibration are to be avoided. Also, the material must have at least moderately high electrical resistance; otherwise eddy-current heating might be appreciable and the induced fields might alter the paths of the orbiting electrons. (It is desirable that, at all times during the acceleration interval, the intensity of induced fields of large area should remain below $1 \%$ of the intensity of the main field.) The inner surface of the chamber must be of conducting material; if it were non-conducting, electrostatic charge might collect here and alter the orbit. 
The chamber must be able to withstand the large amounts of synchrotron radiation emitted approximately tangentially by the orbiting electrons. (The composition of this radiation is discussed in Chap. 25.) The radiation strikes a 1 to $3 \mathrm{~mm}$ high region along the wall of the chamber and delivers about 1 watt of power per linear centimeter, or about 10 watts per sq. $\mathrm{cm}$. at the narrow region of most intense irradiation. The chamber must not suffer chemical change from the radiation, and must dissipate the heat adequately.

The chamber must withstand, also, large amounts of very-high-energy radiations of various kinds, including soft showers produced within the chamber walls. Also, the chamber must be unaffected by moisture and ozone.

The chamber must be strong enough to' withstand atmospheric pressure, and must be unaffected by the moderately high temperature (about $60^{\circ} \mathrm{C}$ ) encountered in normal operation and the even higher temperature (about $80^{\circ} \mathrm{C}$ ) encountered during bake-out. Bakeout is necessary not only on account of the large surface area involved and the complicated internal geometry, but also because, during normal operation of the synchrotron, the chambers are (unavoidably) heated by eddy currents produced by the 60-cycle magnetic field. By baking out the chambers before the magnets are turned on, the Operations Division minimizes the amount of gas released during operation of the synchrotron.

The next section deals with vacuum chambers of oval ring type. In December 1963 about 42 such chambers were in routine use in the ring. Later sections deal with chambers of other types, and also chambers having side tubes for admitting the linac beam, for providing egress for photon beams, and for providing egress for the extracted electron beam.

\subsection{Vacuum Chambers of Oval Ring Type}

Fig. 11.1 indicates the plan-view appearance of a vacuum chamber of oval ring type. Each of its seven-ft.-long straight segments is assembled from large numbers of oval rings of annealed, - low-permeability, Type 316 stainless steel. Each ring is $1 / 2^{\text {" }}$ wide and has a wall $0.083^{\prime \prime}$ thick. The inside height and width of the rings are $1.194^{\prime \prime}$ and 5.544" for the closed-magnet type chamber and 1.484" and 5.394" for the open-type chamber. During manufacture of the core of a chamber, the rings are aligned accurately and are then joined by copper brazing or tack welding. As indicated in Fig. 11.2, the 
successive rings are brazed at alternate ends only, so that deep gaps (nominally about $0.01 "$ wide) exist. The gaps reduce eddy currents, and the brazing provides enough electrical conductivity along a zig-zag path to make it possible for the assembly to be heated electrically for bake-out. The electrical resistance along the entire zig-zag path is 0.65 ohm.

The two segments make an angle of $176.25^{\circ}$ with one another. At the outer end of each there is a 9.000" dia. stainless steel flange, mounted at an angle of $88.125^{\circ}$ (rather than $90^{\circ}$ ) so that the angle between the two flanges is $7.5^{\circ}$ and each flange is perpendicular to the axis of the adjacent straight section tank. The individual flange is not concentric with the segment to which it is attached but is displaced slightly so as to be concentric with the axis of the straight section tank. The working faces of the flanges are smooth and free of radial scratches; thus vacuum-tight seals can be made. (Bellows and seals are discussed in a later section.)

The body of the chamber is wrapped with two layers of $0.007^{\prime \prime}$-thick glass scrim cloth. The cloth is wrapped on the bias, i.e., obliquely, to reduce the tendency for the glass fibers to break where they cross the slots between rings. An extra layer of cloth is included where extra strength is desired and extra space is available, namely the central 4" region near the magnet junction and a short $\left(2 \frac{1}{2}{ }^{\pi}\right)$ region near each flange. The glass cloth is then impregnated with epoxy resin and the assembly is baked.

About $3^{n}$ from each end of the vacuum chamber there is a projecting lug to which bake-out-current wires may be attached; the lugs are used also in grounding the chambers during operation of the synchrotron. Just beyond each lug there is a 0.02 " gap in the stainless steel structure; thus the main body of the structure is electrically insulated from the flanges. The flanges themselves are not baked out.

The finished chamber is introduced gently between the jaws of the magnet and is held in place partly by means of rubber wedges and partly by the flanges and bellows. (The bellows are bolted to the adjacent straight section tanks.) A radial strap of stainless steel prevents the central portion of the vacuum chamber from being forced radially inward by the atmospheric pressure on the flanges; this pressure creates a 200-lb. tension. along the chamber, hence threatens to "straighten out the $V^{\text {" }}$ when and if the epoxy resin becomes hot and compliant.

The contract for construction of the original lot of 58 vacuum chambers of oval ring type was awarded to Youngstown Welding and Engineering Co. That company made 
the stainless steel rings; Ferrotherm Co., a subcontractor, did the copper brazing, and Atkins \& Merrill, Inc., applied the glass cloth and epoxy resin. The first finished chamber was received at $\mathrm{CEA}$ on $3 / 3 / 61$ and the last (the 58th) was received on 10/31/61.

Late in 1963 four more such vacuum chambers for use in open magnets were received and two more for use in closed magnets were received.

Most of the vacuum chambers of the first lot were found to be satisfactory, or practically so. Many required minor repair. A few required major repair and were returned to the manufacturer. However, by mid-February 1962 the entire vacuum ring had been assembled, sealed, and pumped down to $1 \times 10^{-6}$ torr.

During routine operation of the accelerator most of these vacuum chambers have performed well. The typical pressure maintained was $2 \times 10^{-6}$ to $2 \times 10^{-7}$ torr (measured at the entrance port of a high-vacuum pump). (Computations suggest that the pneumatic conductance of one $7-\mathrm{ft}$. segment of the vacuum chamber is about 20 liters/sec; at. : $1 \times 10^{-6}$ torr.) Even when the accelerator was run at $6 \mathrm{Bev}$ and at an intensity as great as $1 / 5$ of the design intensity, a pressure below $2 \times 10^{-6}$ torr was usually maintained. However, chambers that were subjected to especially intense radiation (e.g., by the linac beam) sometimes developed leaks.

During bake-out of the vacuum ring, the chambers are heated to about $80^{\circ} \mathrm{C}$ by means of a 35-ampere, 60-cycle cưrrent from power supplies each of which serves four chambers. The power dissipation per chamber is about 700 watts. Although the epoxy resin has a heat-distortion temperature of about $80^{\circ} \mathrm{C}$, no actual distortion occurs during normal bake-out, provided the radial restraining straps mentioned above are used.

\subsection{Vacuum' Chambers of New Types}

On $1 / 23 / 64$ the first "solid metal side-wall type" vacuum chamber for use in typical locations in the vacuum ring was received at CEA. Fig. 11.3 indicates the cross section of this chamber. The front and back side-walls of the chamber are of stainless steel, and are continuous, or "solid." The top and bottom pieces are of Inconel X; they are slotted, to reduce eddy currents. A wrapping of fiberglass cloth and epoxy resin provides vacuum integrity here. The various metallic members are thick enough, hence stiff enough, so that the overall height of the chamber decreases by an acceptably small amount (less than 0.06") when the device is evacuated. The top and bottom pieces are electrically insulated from the front and back side-walls by fiberglass cloth and cement; thus bake-out 
by electrical heating is easily accomplished. On $3 / 1 / 64$ plans were underway for obtaining many more vacuum chambers of this type.

Another type of vacuum chamber deserving mention is an "external metal foil type," developed at CEA in 1963. It is a modification of a vacuum chamber of oval ring type, and employs a $0.003^{n}$-thick stainless steel foil that lies close against the exterior of the side-wall of the chamber and extends the full length of the chamber. Such a foil may be applied to one side-wall or to both. As indicated in Fig. 11.4, the foil is insulated from the stainless steel core by a thin layer of sprayed ceramic and is sealed along the edges with fiberglass cloth and epoxy resin. Thus the foil constitutes a vacuum-tight, radiationresistant barrier extending along the median-plane region where radiation is most intense. The edge-seals of glass cloth and epoxy resin are located well above and well below the median plane, hence receive relatively modest amounts of radiation. A prototype chamber of this type was installed in the synchrotron in Oct. 1963; it was installed in Magnet 1 where radiation levels (from the linac beam, mainly) have been exceptionally high and conventional chambers have proved to be short-lived (e.g., perhaps 1 week). It performed. without incident for six weeks, then developed a leak in the epoxy coating. On $3 / 1 / 64$ another chamber of external metal foil type was being made ready for use.

\subsection{Injection Chambers}

An injection chamber is used in Magnet 48, just upstream from Straight Section 1 where the beam from the linac joins the synchrotron proper. The chamber has a side-tube, or spout, that allows the linac beam to enter the chamber and join the circular orbit at Straight Section 1:

Two injection chambers of oval ring-type are on hand. In each, internal stainless steel posts are employed (see Fig. 11.5) to strengthen those rings (near the base of the spout) that are especially long and consequently might tend to be deformed excessively by atmospheric pressure. The chamber has the same type of coating of fiberglass cloth and epoxy resin that is discussed in Section 11.2.

The two chambers were ordered in March 1961. The first unit was received on $10 / 27 / 61$, and required some modifications, including (1) welding the free end of each post so that it cannot puncture the coating when the chamber is evacuated and becomes compressed slightly, (2) attaching a thermocouple near the post that: is farthest upstream, so that the synchrotron operator will have prompt warning of any temperature rise such. as 
occurs when the linac beam is misdirected and strikes the line of posts, (3) attaching a third lug, to assist bake-out of the spout. The second unit, incorporating the changes found necessary in the first unit, arrived on 6/4/62.

The two units have been used interchangeably in the ring. The coating on each has suffered much radiation damage. Leaks have developed on many occasions, and each chamber has been repaired many times.

\subsection{Exit Chamhers for Photon Deams}

Requirements. When a bremsstrahlung beam originating within a straight section is to be used outside the vacuum ring, the adjacent downstream magnet must be provided with a vacuum chamber that has an exit spout (terminating, preferably, in a stainlesssteel-foil window of thickness less than $\left.0.003^{\prime \prime}\right)$. The beam then emerges with almost no attenuation. If no exit spout is provided, the beam passes obliquely through the thick wall of the vacuum chamber and suffers much attenuation and broadening; in tests made in 1962 it was found that the intensity of the high-energy photons proceeding straight through the chamber wall is reduced by a factor of about 4 .

In designing a spout for a photon-exit chamber, the designer must take into account the location of the internal target and the direction of the centerline of the photon beam; this direction is the same, of course, as that of the centerline of the beam of high-energy electrons striking the target. Ordinarily, an internal target is situated 1 to $1 \frac{1}{2}$.inches radially inward from the equilibrium orbit and is engaged by the beam-bump procedure explained in Chap.' 16.

Exit chambers for use in open magnets must have slightly different geometry of spout from that appropriate to an exit chamber used in a closed magnet, since the beta functions pertinent to the two types of -magnets differ, as explained in Chap. 3.

The nominal paths of bremsstrahlung beams originating in odd-numbered and evennumbered straight. sections are indicated. in Chap. 24.

Exit Chambers for Use :in Open Magnets. A rib-type photon-exit vacuum chamber for use in open magnets was designed in 1961. Fig. 11.6 indicates the general design. The chamber includes two segments from a standard oval-ring-type chamber, a special spout, and a wide portion consisting of $\frac{1}{2}$-inch-wide segments each strengthened by an internal, transverse rib of stainless steel. The coating of the chamber consists of fiberglass cloth and epoxy resin.: External :longitudinal spars of dielectric material stiffen the assembly. The chamber was completed in mid-1962 and installed in the ring in Sept. 1962. 
On 12/1/63 four such chambers were on hand; three were in routine use. Another unit was on order.

Exit Chambers for Use in Closed Magnets. A photon-exit chamber intended for use in a closed magnet must have a slightly different geometry from that appropriate to an open magnet. As indicated in Chap. 24, the photon beam that emerges from a closedmagnet chamber emerges closer to the equilibrium orbit and the spout must lie closer to the body of the chamber. A suitable chamber employing transverse ribs somewhat similar to those used in the open-magnet chamber was built in 1962 and was installed in the synchrotron in April 1963. A second unit, employing ribs along the entire length, was installed a few months later.

A post-type photon-exit chamber for use in a closed magnet was designed in March 1963 and two such devices were constructed during the summer and fall of 1963 . On 12/1/63 one unit was in successful use in the ring.

On $12 / 1 / 63$ a rib-type photon-exit chamber to be used in conjunction with an internal target mounted in a radially outward position was in an advanced stage of construction. This chamber also is for use in a closed magnet.

\subsection{Exit Chamber for Electron Beam}

By $3 / 1 / 64$ plans for extracting the electron beam were well advanced. Extraction is to be accomplished with the aid of two special magncts mounted in Straight Sections 4 and 5, as described in detail in Chap. 17. The electron beam is to emerge from the synchrotron along a path that lies extremely close to the usual orbit throughout the entire length of Magnet 6 . Since the exit spout on the vacuum chamber and the attached drift tube must also lie close to the orbit, the following straight section tank (\#7) was designed so. as to extend less far upstream, thus allowing room for spout and drift tube. As indicated in Fig. 11.7, the exit chamber designed for use in Magnet 6 is of oval-ring type and has an extra-long spout to make connection with the shortened straight-section tank. The chamber is ribbed along its entire length. It was designed and built in 1962 and 1963 and was installed in the ring in June 1963.

\subsection{Bellows}

The vacuum chambers are joined to the adjacent straight section tanks by means of bellows, which facilitate alignment and allow for thermal expansion. The bellows, made by 
Bellfab Corp., are of Type 316 or 321 austenitic stainless steel, have a nominal diameter of 5.5 to 6.5 inches, and terminate in 3/8-inch-thick, 9.000-inch diameter, stainless steel flanges. The overall length of the assembly is about $1 \frac{7}{2} \pi$, and the range of extension is about $1 / 3^{n}$ or $1 / 2^{n}$. The angular compliance is large, but the shear compliance is less than $0.005^{\prime \prime}$.

\subsection{Vacuum Seals}

It has been the policy at CEA to join vacuum chamber flanges to bellows by means of metal-to-metal seals only; indeed this policy applies to the entire vacuum system of the synchrotron proper. Rubber O-rings would deteriorate rapidly in the high-level radiation fields and would emit vapors that would contaminate the vacuum system.

The O-rings used are of lead, ordinarily. They are made of lead wire $1 / 16^{\text {n }}$ in diameter. The flanges to be joined together in vacuum-tight manner are inspected to make sure that there are no large scratches and, above all, no radial scratches. After the $O-$ ring has been put in place, the screws joining the flanges are tightened firmly. Such a seal is made quickly and lasts almost indefinitely. No evidence of radiation damage has been found. Each time a joint is taken apart and reassembled, a new O-ring is used.

On some occasions O-rings of soft aluminum wire $0.085^{n}$ in diameter have been used (see CEA-TM-26) in conjunction with aluminum spacers 0.064" thick. The screws are tightened until the spacer prevents further tightening; thus the aluminum is compressed to the predetermined extent of 0.021". O-rings of indium have been used on a few occasions.

\section{Main References}

See memoranda, drawings, etc., mentioned above.

\section{Other References}

G. Lanza "Study of a vacuum chamber for synchrotron," CAP-24, 1956.

M. S. Livingston "Vacuum system using oil diffusion pumps," CEA-TM-9, 1957.

B. Stearns "Various suggestions for forming the vacuum chamber," CEA-TM-19, 1957.

B. Stearns "Thermal considerations in vacuum chamber design," CEA-TM-20, 1957. 
R. Hall "Fabrication of O-rings from aluminum wire," CEA-TM-26, 1957.

R. Hall, J. Hess, C. Wakstein "Specification for vacuum chamber sections," 6/8/59.

C. Wakstein "Permeability of type 316 stainless steel," 4/29/60.

D. D. Jacobus "Altered vacuum chamber cross section," 5/20/60.

P. H. Burr and D. D. Jacobus "Specifications for vacuum chamber injection section," $3 / 10 / 61$.

H. Winick "Notes on the vacuum system of the CEA," CEA-TM-83, 1962.

M. Fotino "Specifications for ribbed section of open magnet ejection chamber," 1/15/62.

M. Fotino "Summary of ejection vacuum chamber program at CEA," 2/19/62.

M. Fotino and F. P. Brooks "Specifications for ribbed module of open magnet vacuum chamber," 5/9/62.

M. Fotino "Proposed modification of the CEA vacuum chamber coating," 5/8/63.

D. D. Jacobus "Steel frames for solid wall vacuum chambers," 12/27/63. 


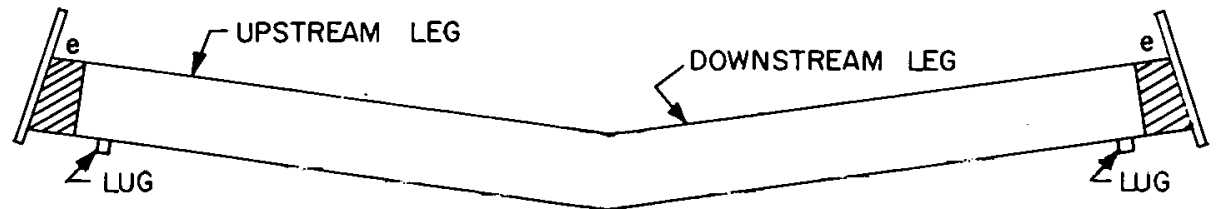

Fig. 11.1. Schematic plan-view diagram of a vacuum chamber of oval ring type. Note that the individual flange is not perpendicular to the leg in question and is not concentric with it. The end portiuns e are electrically isolated from the body of the vacuum chamber.

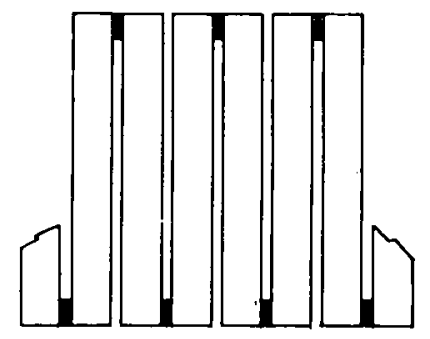

Fig. 11.2. Schematic plan-view diagram of a copper-brazed group of oval rings of stainless steel. The regions where brazing occurs are small, and alternate as indicated to form a zig-zag conducting path.

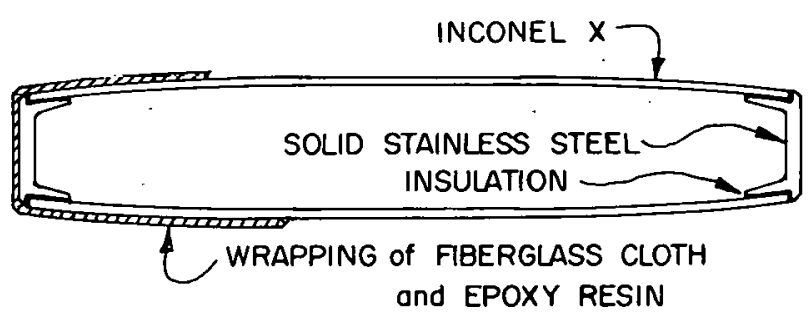

Fig. 11.3. Schematic drawing of cross section of a vacuum chamber of solid metal side-wall type. 


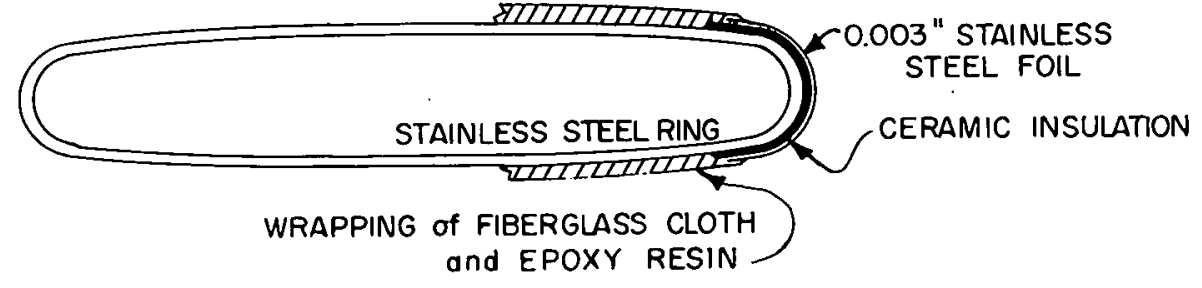

Fig. 11.4. Schematic drawing of cross section of external metal foil type of vacuum chamber.

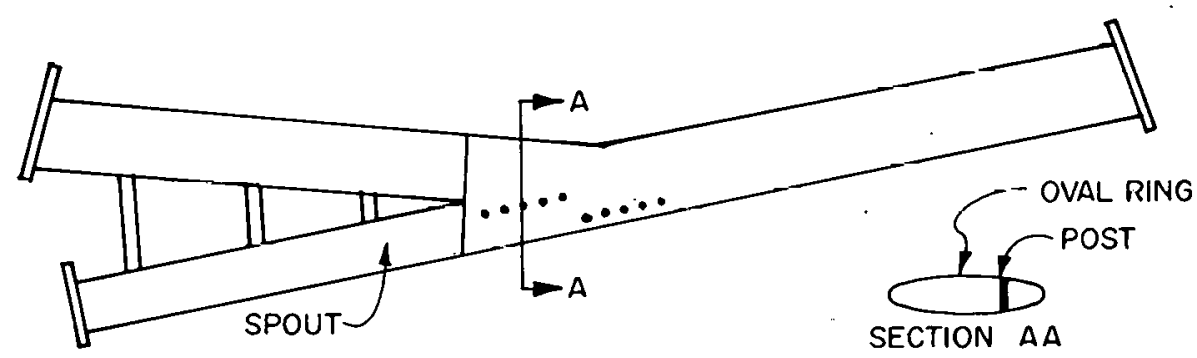

Fig. 11.5. Post-type injection chamber; highly schematic diagram, not to scale.

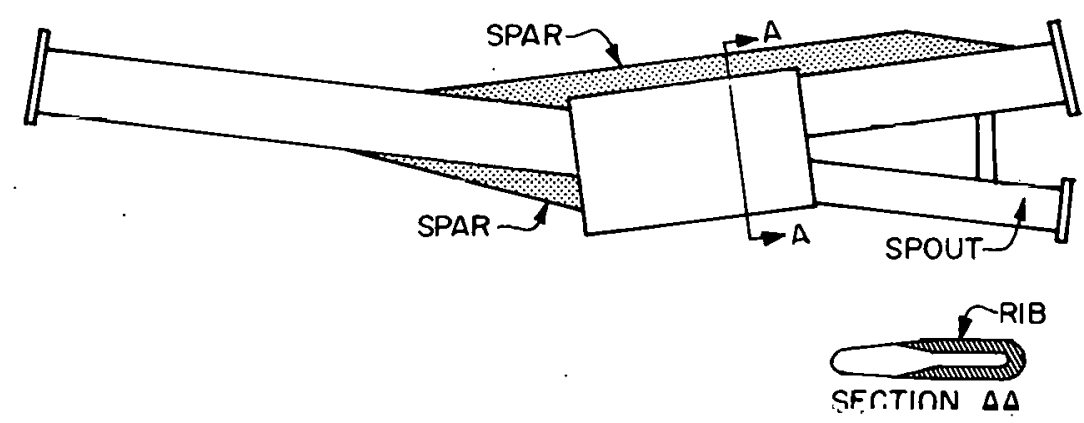

Fig. 11.6. Rib-type photon-exit chamber for use in open magnet. Not to scale.
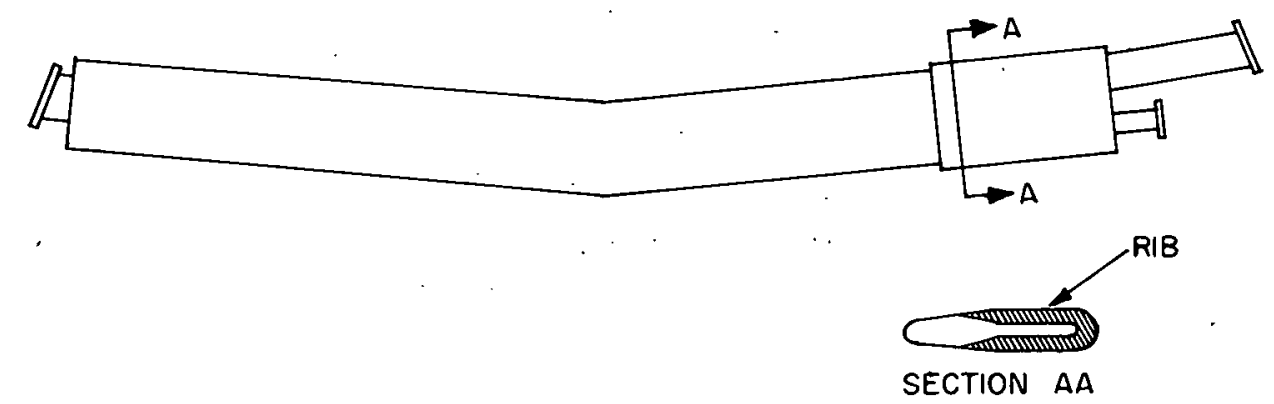

Fig. 11.7. Rib-type electron-ejection chamber for use in an open magnet. Not to scale. 
Chapter 12

\section{Vacuum Pumps}

12.1 Introduction

12.2 Drivac pump

12.3 Varian pump

12.4 Forepump

12.5 .Foreline assembly

12.6 Controls

12.7 Dry nitrogen system

\subsection{Introduction}

As explained in a preceding chapter, it is essential that the average pressure in the vacuum chambers and straight section tanks be kept below about $1 \times 10^{-5}$ torr and desirable that it be kept below about $2 \times 10^{-6}$ torr. Since the high-vacuum pumps are spaced $16 \mathrm{ft}$. apart, the pressure will be perhaps five times higher midway between them than at the pump inlet manifolds; consequently it is appropriate to try to achieve - at the pumps - a slightly lower pressure, such as $1 \times 10^{-6}$ or possibly even $1 \times 10^{-7}$ torr. Mercury diffusion pumps and oil diffusion pumps can meet this requirement. But if they were used, special precautions would have to be taken to prevent the mercury or oil from entering the vacuum system. If mercury or oil did enter, prolonged trouble might result. Also, such pumps require continuous operation of forepumps, and if power failure should occur and all the pumps stopped, air could enter via the forepump outlet lines and might carry large amounts of mercury or oil into the vacuum chambers.

The decision was made in 1960 to employ pumps of the newly-developed titaniumdischarge type. They employ no liquids. Also, after a reasonably good vacuum has been achieved, the outlet lines may be closed, the forepumps can be turned off, and cooled traps are no longer required: If power failure occurs, the vacuum may. remain satisfactory for some time.

\subsection{Drivac Pump}

The heart of the CEA high-vacuum pumping system is a set of Consolidated Electrodynamics Corp. three-electrode, high-voltage, titanium-discharge, "Drivac" pumps having a rated speed of 300 liters per second at $10^{-6}$ torr. Before the decision was made (in 1960) to employ pumps of this make, many other designs of titanium-discharge pump were 
considered. Several were tested thoroughly. See, for example, CEA-TM-54 and CEA-TM-58. A small, three-electrode, high-voltage-discharge pump made by Consolidated Electrodynamics Corp. was studied especially carefully and was found to be superior to other existing types in several respects, including these: it was less prone to occasional release of large quantities of gas, and it appeared capable of pumping even the noble gases (argon, for example) with adequate speed. An order was placed for 50 pumps of this general design, but of larger capacity, namely 300 liters/sec at $10^{-6}$ torr.

Fig. 12.1 shows the cross-section of the Drivac pump, and Fig. 12.2 shows how the pump is mounted. Surrounding the central vertical inlet chamber are three radial galleries in which the actual pumping occurs. A thick, egg-crate structure of stainless steel (the anode) occupies the center of the gallery and is kept at aboul 3500 volts. Flanking it are thin honeycomb structures (cathodes), also of titanium; these are kept at -3500 volts. Beyond these are the gallery walls, of stainless steel; these are at ground potential. The galleries are pervaded by an 1800-gauss magnetic field produced by heavy, externally mounted, permanent magnets of Alnico.

During normal operation of the pumps, gas that finds itself in a straight section tank may pass downward, via the port at the bottom, into the central chamber of the Drivac pump situated just below. Thence it passes into the galleries. Gas within or close to the egg-crate anode becomes ionized by electrons that travel rapidly back and forth along helical paths concentric with the lines of magnetic flux. (The ionization process serves also to maintain the population of free electrons.) The ionized gas molecules are attracted (accelerated) toward the nearby titanium cathode; most of them pass through it, and are decelerated, and then strike the stainless steel wall. Here most of them are "gettered," or chemically adsorbed, by the titanium coating on the wall. Some of the ionized gas molew cules, while traveling at near-maximum speed, strike the cathode and sputter titanium metal from it onto the nearby wall. Thus the titanium coating on the wall is continually augmented, ready to absorb additional quantities of gas. It is believed that the noble gases present in the pump are not gettered in the usual sense but are physically buried by the steady rain of titanium.

Each pump is enclosed in an external metal basket which provides help to prevent people from touching the high voltage terminals and prevents iron objects, from being attracted to the large Alnico magnets. Each Drivac has its own d.c. power supply, which is a Type 923-0000. supply made by Varian Associates. It includes two portions, one supplying 
current at about 4000 volts and the other at -4000 volts. About 100 semi-conductor diodes are included. The current varies almost linearly with the gas pressure in the Drivac, if the pressure is below $10^{-5}$ torr; by noting the current the operator can infer the pressure. A current of $8 \mathrm{ma}$ implies a pressure of about $1 \times 10^{-6}$ torr. When the pump is started (i.e., after the forepumps have reduced the pressure to about $10^{-3}$ torr) the current is nearly as great as the short-circuit current, which is about $500 \mathrm{ma}$. Two circuit breakers are included in the $208 \mathrm{v}, 3$-phase, input side of the individual power supply: a 30-amp. breaker employed during start-up, and a 5-amp. breaker employed during normal operation. The power supplies were purchased from Varian Associates early in 1961.

If the Drivacs are to perform reliably for days or weeks, they must be re-stabilized once or twice a week. This is done by heating the vacuum chambers to about $80^{\circ} \mathrm{C}$ (by means of the bake-out procedure described in the previous chapter) with the Drivacs on, the excitation of the 48 magnets off, and the forepumps valved-off. Heating the chambers causes them to release gas, and the resulting increased load on the Drivacs causes their interior surfaces to heat up and re-emit some of the previously pumped gas. The pressure rises to perhaps $5 \times 10^{-5}$ torr and then gradually decreases by one or two orders of magnitude as the gas is readsorbed by, or reburied in, the titanium in more. stable manner: The whole process takes about two hours.

On the whole, the Drivacs have performed well. In recent months (and in the absence of known leaks in the vacuum chambers) they have maintained average pressures of about $10^{-6}$ torr in the vacuum ring. However, in 1961 and 1962 some modifications to the structures.within the galleries had to be made, to avoid occasional short-circuiting by growth of metallic whiskers or by warping of the electrodes. A number of minor difficulties remained, and it was occasionally necessary to remove a pump and overhaul it. Fortunately, it was usually possible to operate the synchrotron even when several pumps had developed troubles and had to be shut off.

\subsection{Varian Pump}

In 1962 and early 1963 three Varian Associates VacIon No. 911-5020 high-vacuum pumps rated at 400 liters/sec were purchased. One was put to use promptly at Straight Section 2. A second was later put to use in Straight Section 1. All have performed well. Varian Type 921-0005, 7300 v. power supplies are used. 


\subsection{Forepump}

Adjacent to each of the 48 high-vacuum pumps there is a Cenco Hypervac \#25 forepump, capable of achieving a vacuum of $10^{-3}$ or $10^{-4}$ torr under favorable circumstances. Eighteen of these were ordered in March 1960 and 32 more were ordered in Feb. 1961. By Dec. 1961, 48 had been tested and installed in the circular tunnel. The pumps run directly off: the 60-cycle, $208 \mathrm{v} . ; 3$-phase line; and are turned on and off individually, locally. They have performed well.

\subsection{Foreline Assembly}

Associated with each forepump there is a foreline assembly. This includes:

A Hughes Aircraft Company No. RF-75K ionization-type high-vacuum gage.

An outlet tube to which a helium-type leak detector may be connected; also a valve for closing off this tube.

A main shut-off valve; this remains closed during normal operation of the synchrotron.

An intermediate-range vacuum gage for measuring the rough vacuum between highvacuum pump and forepump.

A trap that may be filled with liquid nitrogen shortly after the forepump is turned on.

A solenoid-actuated vent valve; just before the forepump is shut off, this valve aulv= matically admits air to the foreline.

The foreline assemblies were made by Accurate Aircraft Parts, Inc., in mid-1961 and were installed in the ring in the fall of that year. They have performed well.

\subsection{Controls}

The forepumps are turned on individually, locally. The high-vacuum pumps are controlled mainly from the Vacuum Control Area in the Power Bldg., and may be turned on and off, individually or in groups of six. Also, they may be turned off in groups of six locally, in the circular tunnel.

The Vacuum Control Area includes the following indicators:

Milliammeters incorporated in the 48 power supplies. Each meter indicates the current drawn by one pump. Knowing the current, an operator can infer the pressure in the pump. 
Red pilot lights indicating the satisfactoriness or unsatisfactoriness of the 48 power supplies; the red lights are arranged in a circular pattern corresponding to the pump locations in the circular tunnel. A given light is lit whenever the current drawn by the corresponding power supply exceeds a "danger threshold" of about $60 \mathrm{ma}$ or whenever the voltage output of the supply falls as low as 500 volts.

A buzzer that sounds whenever a red light is lit and whenever a power supply voltage falls below 500 volts. By pressing an "acknowledge" button the operator can turn off the buzzer.

An amber light that flashes under the same circumstances that activate the buzzer.

The Control Room includes a red warning light that is lit whenever a red pilot light in the Vacuum Control Area is lit and a milliammeter that may be connected to any one power supply.

The RF Control Room includes milliammeters that are connected to the power supplies associated with high-vacuum pumps situated beneath $\mathrm{rf}$ cavities - so that the pressures in these cavities can be ascertained conveniently and quickly.

The procedures used in starting the vacuum pumps and baking out the vacuum chambers are described in CEA-TM-83.

\subsection{Dry Nitrogen System}

When the vacuum system must be opened (for example: to permit removing and repairing a vacuum chamber, or to permit installing a target in a straight section tank) it is filled with dry nitrogen, rather than ambient air, in order that moisture and oxygen will be excluded.

Nitrogen gas is stored at high pressure in steel cylinders in the Power Bldg., and flows, via suitable tubes, pressure reducers, and regulating valves, to the vacuum ring. CEA-TM-83 describes the system.

\section{Main References}

See memoranda, etc., mentioned above.

\section{Other References}

M. S. Livingston and A. Koehler "Vacuum system using oil diffusion pumps," CEA-TM-9, $5 / 22 / 57$. 
M. D. Kostin "Gas scattering in a strong focusing synchrotron; single scattering theory," CEA-66, 11/19/58.

J. Hess "Varian pump report," CEA-TM-36, 8/28/58.

J. Hess "Discussion of the Vapor-Ion pump with regard to the CEA vacuum system," CEA-TM-47, 4/1/59.

J. Cerino, J. Hagopian, J. Hess "Pellet feed for getter-ion-type pump," CEA-TM-49, 8/6/59.

D. S. Edmonds, Jr. "A proposed power supply for a 300 -liter/sec version of the Drivac pump," CEA-TM-55, 1/7/60. 


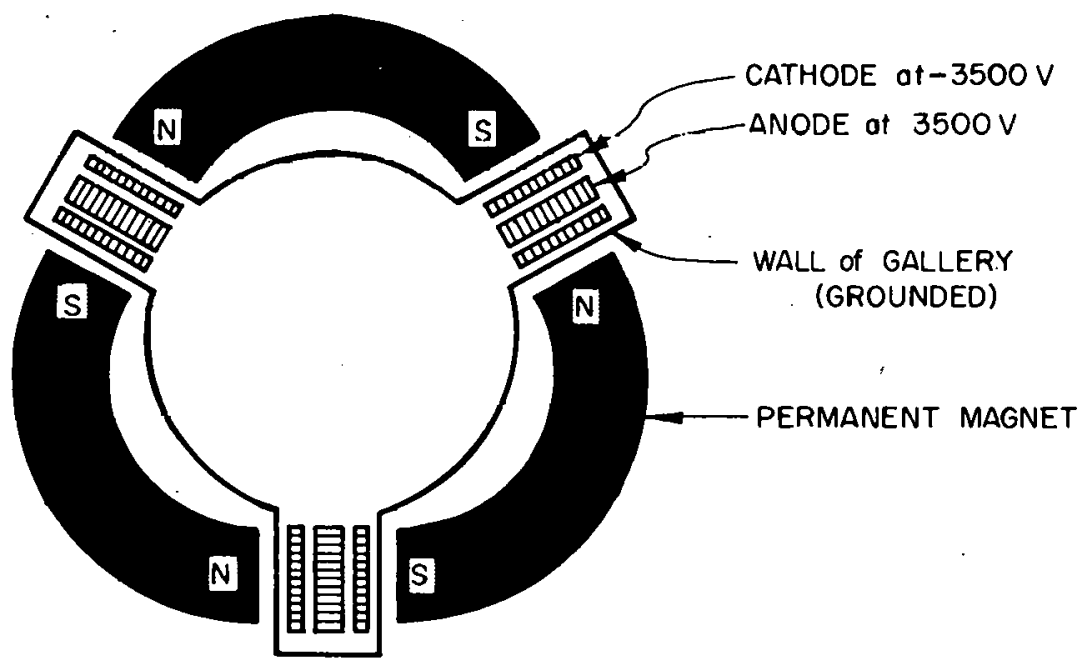

Fig. 12.1. Horizontal cross section (schematic) of Drivac 300 liter/sec high-vacuum pump, showing the three radial galleries each of which contains an egg-crate anode at positive potential flanked by honeycomb cathodes at nega- . tive potential. The permanent magnets produce magnetic fields that are normal to the galleries. 


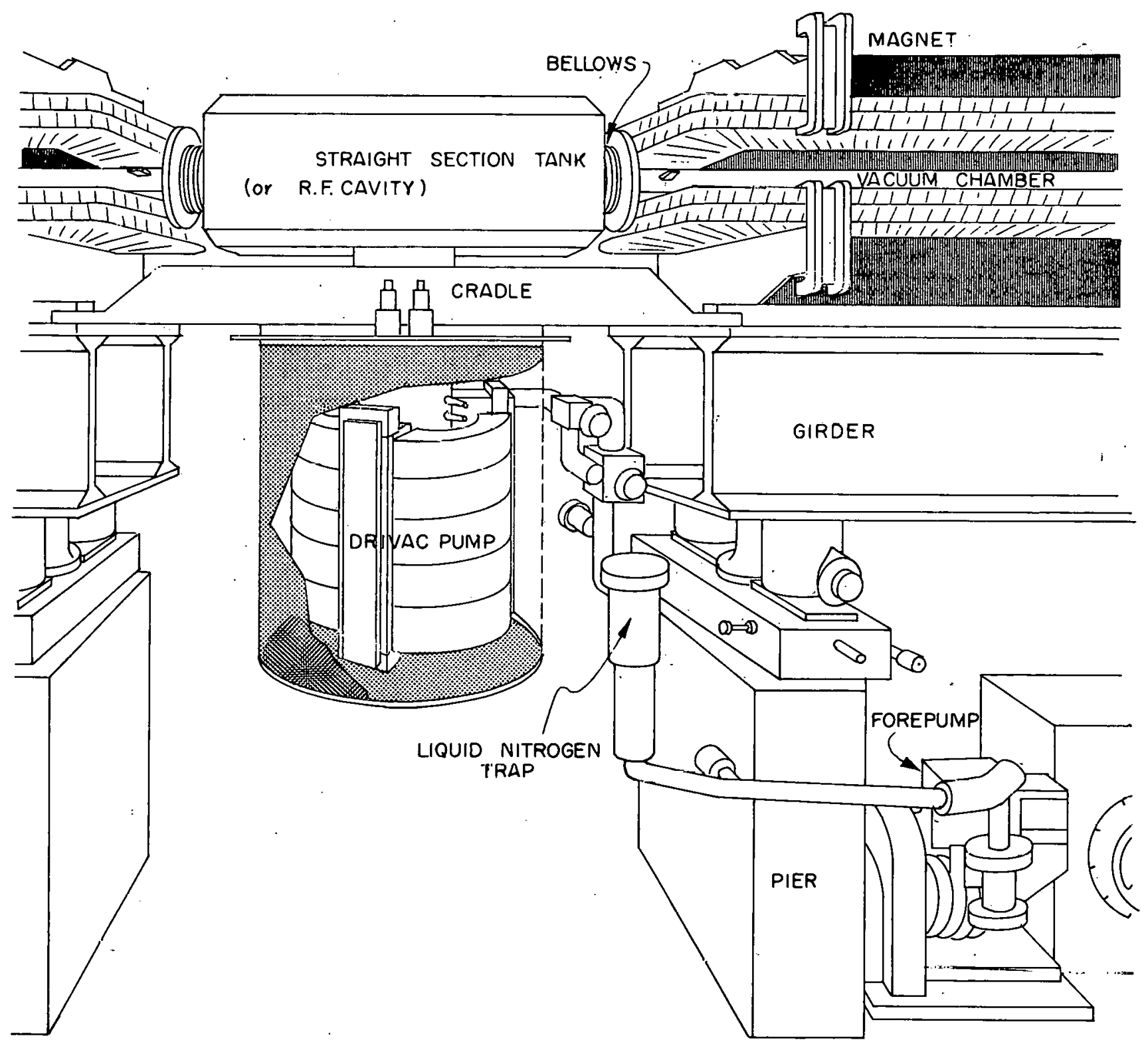

Fig. 12.2. Typical pumping station. 
13.1 Straight-section tank of standard type

13.2 Straight-section tank for housing pick-up coils

13.3 Other straight-section tanks; also valves therein

13.4 Supports for straight-section tanks

13.5 Magnetic shielding

13.6 Aligning the tanks

\subsection{Straight-Section Tank of Standard Type}

The typical, standard, straight-section tank has two main functions: it provides a vacuum path between the two adjacent vacuum chambers, and it supports a high-vacuum pump and serves as manifold therefor. Some tanks perform the additional function of supporting pick-up coils; these tanks have been specially modified and are discussed in the following section.

Some of the standard tanks contain one of the following: magnetic inflector, target, scraper, magnet used in ejecting the electron beam, mirror used in observing synchrotron radiation.

As shown in Drawings S-16M-94B and S-16M-95A, the standard tank consists essentially of a non-magnetic stainless steel cylinder about $10^{\prime \prime}$ in outside diameter and $28^{\prime \prime}$ long. The axis of the cylinder is horizontal, lies in the mechanical median plane of the set of 48 magnets, and is $1.143^{\prime \prime}$ from the apex of the pertinent corner of the 96 -sided polygon, as indicated in Fig. 13.1. The intent was that the axis should coincide with the equilibrium orbit of electrons passing through the tank. Thus the location corresponds exactly to that of an rf cavity; see Chap. 10.

At each end of the tank there is a $9.000^{\prime \prime}$-diameter flange to which bellows may' be attached (cf. Chap. 11). The upper edge of the flange is nominally (11.250" $-4.500^{\prime \prime}$ ) or $6.750 "$ below the top of the magnet. At the bottom of the tank there is a vertical tube (with $6^{n}$ inside diameter) that projects about $8^{\prime \prime}$ downward and terminates in an $11^{\text {"r}}$-diameter flange to which a high-vacuum pump may be attached. On the side farther from

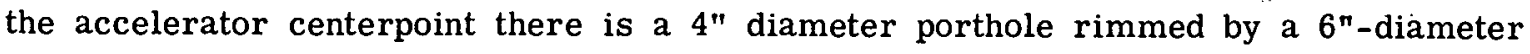
flange, and on the opposite side there are two such ports $12^{n}$ apart on centers. At the top there is another such port. On the bottom of the tank there are two U-shaped brackets that rest on the heavy aluminum support described in a later section. 
The construction of the tank is described in "Specification of Pick-Up Electrode Cavities" by D. D. Jacobus, 9/2/60. A total of 34 tanks were made - by Hicks Co., just prior to March 1961. Inspection of these tanks showed that in many instances the end flanges were neither flat nor parallel to one another; accordingly they were sent to Accurate Aircraft Parts, Inc., to be remachined (in the summer of 1961). Eighteen were later modified for use with pick-up coils, as explained in the next section.

\subsection{Straight Section Tank for Housing Pick-Up Coils}

The straight section tanks that house pick-up coils for monitoring beam intensity and beam displacement are modified versions of the standard tank described in the previous scction.

As indicated in Drawing $S-16 \mathrm{M}-126-\mathrm{E}$, the end flanges are of heavier type and are specially machined to support the pick-up coil assemblies rigidly and accurately, with the aid of dowel pins.

Also, two survey mounts, or pedestals, are attached at the top. The pedestals are machined accurately, and the heavy flanges are machined in accurate relationship to them. Thus the surveyor, on aligning the pedestals accurately with respect to the magnets, knows that the flanges - and hence the pick-up coils also - are in accurate relationship to the magnets. The upstream pedestal is only 1.750" wide, but the downstream pedestal, intended for use in determining the transverse levelness of the tank, is $6.000^{\text {" wide. The }}$ top of each pedestal is nominally $6.656^{\prime \prime} \pm 0.005^{\prime \prime}$ above the centerline of the tank. Measurements made in 1962 showed that the relationship between pedestals and dowel pins had an $\mathrm{rms}$ variability of about $0.015^{\prime \prime}$. (Beam height measurements made early in 1963 with the aid of pick-up coils suggested either that the beam was about $0.05^{n}$ too high in most of the tanks or that, for some reason, the pick-up coils were situated too low by this amount.)

The special machining, etc., required for these tanks was done by Accurate Aircraft Parts, Inc., in the fall of 1961. A total of 18 tanks were processed. Chapter 14 lists the straight sections that contain such tanks.

\subsection{Other Straight Section Tanks; Also Valves Therein}

The tank situated immediately downstream from the. electron-ejection-type vacuum chamber (i.e., Tank 7 , on $3 / 1 / 64$ ) is of special design, such that interference with the 
emerging electron beam is avoided. The upstream end of the tank is very short and slender. The body of the tank is only $12 \frac{1}{2}$ " long. Attached to the downstream end is an auxiliary section about $7 \frac{1}{2}{ }^{m}$ long, which contains a manually operated valve.

The tank used in Straight Section 14, in electron scattering experiments, is of very special type. The central portion consists of a vertical cylinder of large diameter, for housing a remotely-controlled array of targets. The radially outer portion of the cylinder includes a long horizontal window of thin mylar; for allowing scattered particles to emerge with little scattering. At the upstream and downstream ends of the assembly there are large; manually-operated gate valves. The equipment was built 'in the Cyclotron Laboratory and was installed in the ring on $9 / 27 / 62$.

In August 1963 a special tank fitted with a $4 \frac{1}{2}$-inch manually operated gate valve was installed in Straight Section 43. The valve was made by Wright Associates, and employs an organic O-ring.

\subsection{Supports for Straight Section Tanks}

The straight section tanks, like the rf cavities, rest on cast aluminum supports that bear on the adjacent magnet girders. But whereas the rf cavities rest on recessed, cradle-like supports, the straight section tanks rest on supports that project upward (about $5 \frac{7}{16} "$ ) above the plane of the girder tops. The supports are held by a tongue at the upstream end and a pin at the downstream end, to avoid constraining the positioning of the girders. The tank is held securely to the support by means of four bolts. The effective lengths of the bolts are variable; thus the height of the tank can be adjusted, and the bolts are situated in radial slots so that the radial position can be changed.

Drawings $\mathrm{E}-16 \mathrm{M}-79 \mathrm{~A}$ and $\mathrm{E}-16 \mathrm{M}-80 \mathrm{~A}$ indicate the designs used for supports situated just downstream from an open magnet or a closed magnet respectively. Some modifications of the ends were made to avoid interference with the guide rails on the girders.

\subsection{Magnetic Shielding}

As explained-in CEA-TM-77, the region occupied by a straight section tank tends to be pervaded by stray magnetic fields due to (a) the earth, (b) neighboring synchrotronmagnets, (c) the Alnico magnets associated with the adjacent high-vacuum pump, and (d) the nearby Harvard cyclotron. Accordingly, magnetic shielding has been provided for most 
of the tanks. If the shield has suitable geometry and is made of metal having high initial permeability and a high saturation level, fields of small to moderate magnitude tend to run inside this metal and not in the region enclosed by the shield.

As shielding material, 20-mil-thick Co-netic metal AA, made by Magnetic shield Division of Precision Mica Corp., is used. The "mill-annealing" which the metal had been subjected to as purchased proved to be insufficient. Consequently the CEA had the metal re-annealed with great care - after the metal had been cut and bent to proper shape. The shield was heated to $1850^{\circ} \mathrm{F}$ in an anhydrous hydrogen atmosphere, kept at this temperature for several hours, then cooled at the rate of $100^{\circ} \mathrm{F}$.per hour. Thereafter it exhibited high initial permeability, high saturation level, and low residual magnetism.

The shield designed for use with a standard straight section tank consists of a long cylinder that fits inside the tank. A hole cut in the cylinder allows gas to pass downward into the high-vacuum pump. In some shields, holes have been cut adjacent to the various side ports of the tank.

The shield designed for use with a pick-up coil tank is mounted outside the tank, since there is not enough room inside. Being mounted outside, and having also various holes cut adjacent to the ports, this shield tends to be less effective. Consequently two layers of Co-netic metal are employed.

The shields were designed and fabricated at CEA. The annealing was done elsewhere.

\subsection{Aligning the Tanks}

To determine whether each end of each tank has the correct height and correct radial position relative to the adjacent magnets, the surveyor employs a yoke-shaped gage. The yoke rests on a guiding platform which in turn rests on top of the magnet and bears against the $1 / 2^{n}$-high shoulder there in definitive manner. With the platform held fixed, the yoke may be slid along parallel to the axis of the tank; thus it may be used to measure the position of any of the three flanges (vacuum chamber flange and bellows flanges) adjacent to the tank-end in question. The yoke is designed to clear a perfectly aligned $9.000^{\prime \prime}$ dia. flange by $1.000^{\prime \prime}$ at the top and 1.010" at either side. The platform may be adjusted for use on either a closed or open magnet. W. Shurcliff's memorandum of 4/25/61 describes the gage in detail. (The same gage is used in aligning an $r f$ cavity, although here the flange diameter is 11.000 " so the three clearances at the top and at the side are $0.000^{\prime \prime}$ and $0.010^{\prime \prime}$ respectively.) 
To verify the transverse levelness of the tank, the surveyor lays a machinist's level on the $6^{n}$-wide pedestal.

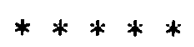

\section{References}

See specifications, drawings, etc., mentioned above. 


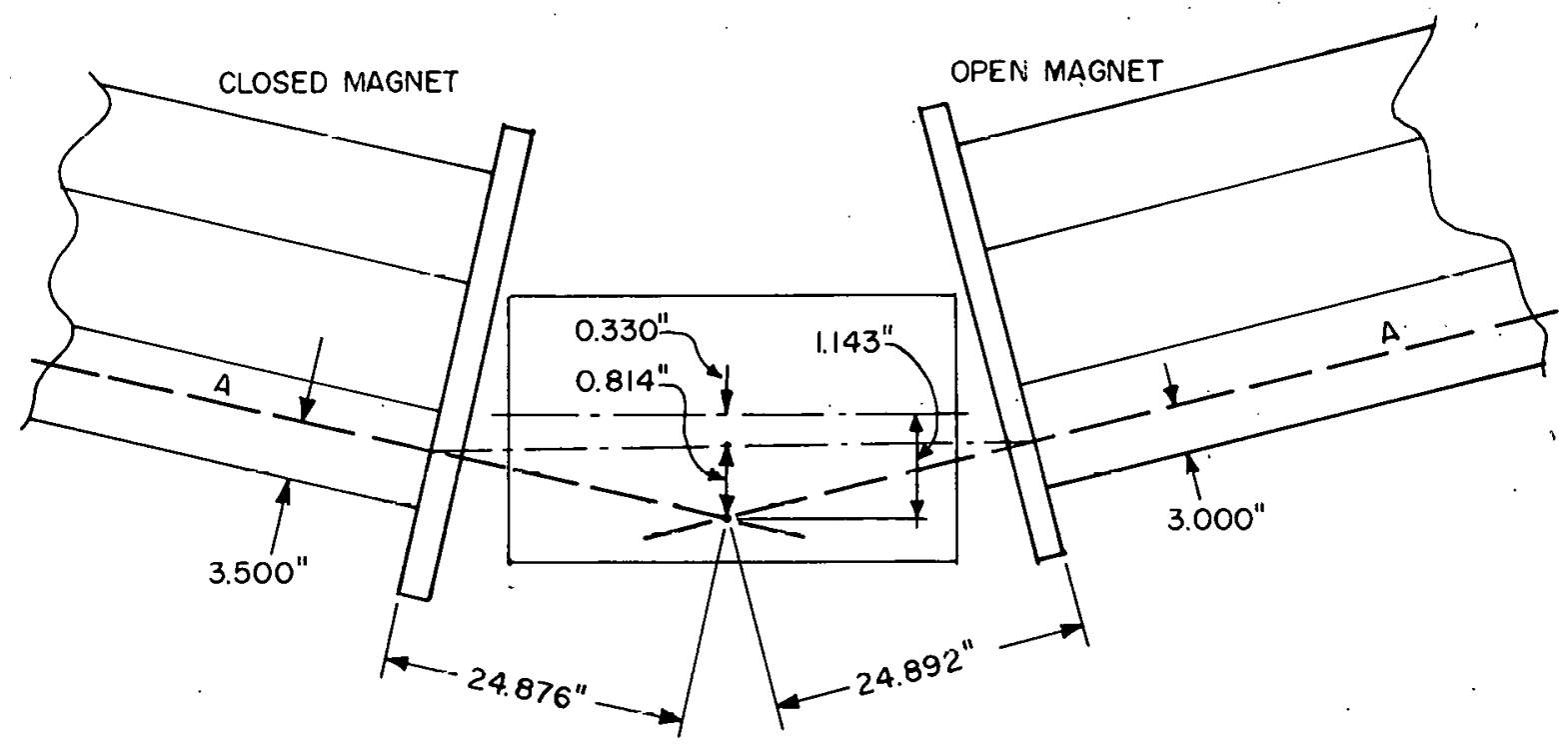

Fig. 13.1. Schematic diagram showing the original nominal orientation of the centerline of a straight section tank or $\mathrm{rf}$ cavity relative to the pertinent sides $A$ of the basic 96 -sided polygon. On $7 / 12 / 61$ the situation was changed slightly: the closed magnets were moved radially inward by $0.045^{\prime \prime}$ and the open magnets were moved radially outward by 0.045 ". Thus the numbers $3.500^{\prime \prime}$ and $3.000^{\prime \prime}$ were changed to $3.455^{\prime \prime}$ and $3.045^{\prime \prime}$ respectively. 


\section{Chapter 14}

\section{Monitors of the Orbiting Beam}

14.1 Introduction

14.2 Beam intensity monitor of resonant cavity type

14.3 Beam intensity monitor of standard pick-up coil type

14.4 Beam intensity monitor of "portable" pick-up coil type

14.5 Monitor of horizontal displacement

14.0 Munltur uf vertical dispiacement

14.7 Monitor employing synchrotron radiation

\subsection{Introduction}

Tuning the accelerator is an important, difficult, and frequent task. To accomplish the task, the operator must have accurate information concerning the orbiting beam. Specifically, he must know:

The intensity of the beam at any moment during the acceleration interval.

The horizontal (radial) displacement of the beam at many positions along the orbit.

The vertical displacement at many positions along the orbit. Possessing this information, he can make the adjustment needed (see Chap. 27) and, by trial and error, achieve a beam of satisfactory intensity. There is also the possibility of employing the information in a computer program for converting displacement values into values of necessary corrective action. The corrective action might consist, for example, of altering the currents in the horizontal and vertical steering coils that flank the rf cavities; see CEA-78 and CEA-89. Several such computer programs were carried through successfully early in 1962.

\subsection{Beam Intensity Monitor of Resonant Cavity Type}

The most sensitive monitor of average current carried by the orbiting electrons is a monitor that employs, as sensor, a spare rf cavity. The cavity is tuned to approximately the rf frequency; and the $Q$ of the (loaded) cavity is about 600; accordingly a fairly intense electromagnetic field is induced in the cavity by the orbiting electrons (which, of course, are bunched with a spacing that corresponds to the $\mathrm{rf}$ frequency). A fraction of the cavity power is transmitted by coaxial cable to the Control Room, where it may be 
put to various uses. For example, it may be used to actuate a current-vs.-time meter; the time constant is about $10 \mu \mathrm{s}$, and a precision of the order of $0.1 \%$ of the accelerator design intensity is achieved. The current may be rectified, amplified, and fed to a current integrator, i.e., a milliampere-hour meter.

\subsection{Beam Intensity Monitor of Standard Pick-Up Coil Type}

When the accelerator is being started and the operator wishes to see how much current is present in each of the first few turns, he employs a beam intensity monitor that includes magnetic pick-up coils. Such sensing devices may be installed in any of various straight section tanks.

In 1960 and early 1961 the relative merits of magnetic pick-up coils and electrostatic pick-up electrodes were debated. The issue was settled in May 1961, when the two types of sensors were tried out - in series - in the $25 \mathrm{Mev}$ beam from the linac. The signals produced by the coils were large and stable, but those produced by the electrodes fluctuated wildly as a consequence of collection (or discharge) of stray electric charge.

The feasibility of using magnetic pick-up coils (or of using electrostatic pick-up electrodes) stems from the fact (cf. Chap. 9) that the circulating train of electrons does not have a length equal to that of the orbit; the length is only about $5 / 7$. or $6 / 7$ as great, due to the time interval of about 0.1 or 0.2 microsec required to turn off the magnetic inflecting field. The train is about $600 \mathrm{ft}$. long, and there is a gap about $150 \mathrm{ft}$. long. Each time the train (or the gap!) passes by a pick-up coil, it induces therein a voltage proportional to di/dt. The system is analogous to an air-core transformer having a oneturn primary (corresponding to the electron beam). and a many-turn secondary. By resistively integrating the voltage induced in the pick-up coil, we obtain a signal that is proportional to the instantaneous current in the beam.

The device includes two coils, one above the beam and one below it. Each has the form of a coreless, rectangular-cross-section solenoid, the axis of the solenoid being horizontal and perpendicular to the beam.

CEA-89 describes the design of the coils in considerable detail. Here it is sufficient to say that the geometry is such that the total induced voltage is almost independent of any small vertical displacement of the beam centerline from the equilibrium orbit and almost independent of small horizontal displacements, such as the 0.3-inch radially-outward shift that occurs as the electron energy increases from $0.03 \mathrm{Bev}$ to $6.0 \mathrm{Bev}$. The clear aperture between coils is $1 \frac{1}{2} "$ high and $4 "$ wide. 
Epoxy coated copper wire is employed; it is wound on lava-stone insulators that have been machined and then heat-treated to produce ceramic-like properties. The d.c. resistance of the coil system is $40 \mathrm{ohms}$, the inductance is $2800 \mu \mathrm{h}$, and the capacitance is $54 \mathrm{pf}$.

To suppress signals resulting from electrostatic pick-up due to the passing train of electrons (and erratic collection or release of stray charge such as is prevalent during and immediately following injection), various precautions were taken. The coils were.. wound symmetrically, and electrostatic shields were provided. To reduce parasitic oscillations, the wires were divided into groups each provided with a damping resistor. To permit retaining a large bandwidth, the capacitance-to-ground was kept very low.

The amplifier, also, is described in CEA-89. See also Drawing :B-16E-18F. The amplifier is of differential type: it greatly amplifies the voltage difference between the two leads from the coil system and provides almost no amplification for the fluctuations in potential of the coil system as a whole. Thus spurious signals from electrostatic charge are suppressed. The factor of common-mode-rejection is about 100. The closed loop gain is 140 , the percent drop for $0.6 \mu \mathrm{s}$ pulse is $6 \%$, the rise time (10 to $90 \%)$ is $28 \mathrm{~ns}$, the maximum undistorted output is $\pm 2.0 \mathrm{v}$, the percent gain control is $10 \%$, the equivalent $\mathrm{rms}$ noise voltage referred to the input is $26 \mu \mathrm{v}$, and the output impedance is 50 ohms. Very-high-gain tubes are used. Because microphonics proved to be a major limitation, elaborate shock-mounting was. provided. The amplifier is situated only a few feet from the coils themselves. The average length of coaxial cable carrying the signal to the Control Room is $400 \mathrm{ft}$.

The multi-megacycle-bandwidth signal reaching the control room is presented on an oscilloscope, the horizontal sweep being triggered by a signal from the peaking strip (cf. Chap. 23). Any desired delay and expansion may be accomplished. The individual trace consists of a series of pips, and by regarding the peak of the pip, the maximum undershoot, and the base line, the operator can find not only the instantaneous current . but also the value of current averaged over several microseconds.

\subsection{Beam Intensity Monitor of "Portable" Pick-Up Coil Type}

Several "portable" pick-up coil systems are available for monitoring beam intensity. These devices are much like those described in the previous section, but employ smaller. coils. The coils are so small that they may be inserted in a straight section tank via one 
of the side ports, i.e., without the need for moving the tank as a whole. The responsivity is the same as that of the standard units.

On $3 / 1 / 64$ two or three of these devices were in use in the ring.

\subsection{Monitor of Horizontal Displacement}

Magnetic pick-up coils are employed also in sensing the horizontal (radial) displacement of the centerline of the electron beam. On $3 / 1 / 64$ sixteen sets of coils were in use: they were situated in Tanks $2,5,8,11,13,17,20,23,25,29,31,35,38,41$, 44 , and 47 .

The displacement sensors, like the intensity sensors, succeed only because of the gap in the orbiting train of electrons. Again, the signals are integrated, amplified, and transmitted to oscilloscopes in the Control Room. The amplifiers used, and the precautions taken to avoid trouble from stray charge, microphonics, etc., are almost identical to those described in the previous section. The operator must remember that the signals are proportional to the product of horizontal displacement and beam intensity.

CEA-89 describes the design of the coil system. Only a brief summary is requirec here. Each coil system has much resemblance to a pair of Helmholtz coils. One of the coils is above the orbit, and the other below the orbit; a $1 \frac{1}{2} n$ gap separates them. The geometry of the system was carefully chosen so that the output signal, although strongly dependent on the horizontal position of the beam, is almost independent of the vertical position; specifically, if the vertical position is too high by $2 \mathrm{~mm}$, the output signal for a typical given horizontal displacement is changed by only about $2 \%$.

The responsivity of the system is high. When a $21 \mathrm{ma}$ beam is displaced horizontally by 1 inch, the output signal measured at the coils is $4 \mathrm{mv}$. Under favorable circumstances a displacement as small as 0.01 inch is detectable. However, electrical noise is troublesome - at the time of injection - to pick-up coils situated near the linac or magnetic inflector, making it difficult to measure less-than-0.05-inch displacements at such locations during the first few turns.

The absolute accuracy of the measurement depends, of course, on the accuracy with which the coil system is positioned relative to the equilibrium orbit. Positional accuracy within 15 or 20 mils was achieved by building the coil system to close mechanical tolerances, mounting the system on a specially machined end-flange of the straight section tank (see Chap. 13), providing external survey mounts machined in accurate relationshj 
to those flanges, then aligning the tank carefully with respect to the magnets with the aid of specially designed alignment fixtures (described in W. Shurcliff's memoranda of 4/25/61 and 5/18/61). The special machining was done by Accurate Aircraft Parts Co. in Sept. 1961 in accordance with Drawings S-16M-126C, D-16M-128C, D-16M-131A.

\subsection{Monitor of Vertical Displacement}

Mounted integrally with the coil system for sensing horizontal displacement is a coil system for sensing vertical displacement. The design, indicated in CEA-89, is such that the output signal, although strongly dependent on the vertical position of the beam, is almost independent of the horizontal position. Specifically, if the beam is situated too far radially outward by $0.5^{\prime \prime}$, the output signal for a given vertical displacement is changed by only about $7 \%$. The sensitivity of the monitor is such that when a 21 ma beam is displaced 0.5 inch vertically, the output signal at the coils is $5 \mathrm{mv}$. A displacement of only 3 mils vertically is barely detectahle. However, the uncertainty in absolute vertical position of the coils themselves is of the order of 10 mils. The free aperture left by the coils is $1 \frac{1}{2} "$ by $4 \frac{1}{2} "$.

\subsection{Monitor Employing Synchrotron Radiation}

The shape and size of the cross-section of a beam of 1 to $6 \mathrm{Bev}$ electrons may be monitored visually by observing the synchrotron radiation. (The radiation itself is discussed in Chap. 25.) A plane, front-surface, aluminized quartz mirror mounted in Straight Section 26 reflects synchrotron radiation out of the straight section tank via a glass window and along Tunnel 26 to an observation station in the Power Bldg. basement. The radiation that is viewed originates near the center of the downstream half of Magnet 25 and is emitted, of course, approximately tangentially. The observer, situated $45 \mathrm{ft}$. from

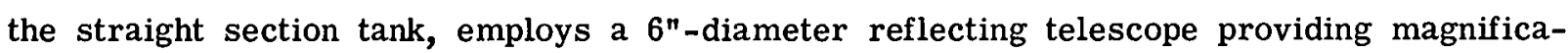
tions of 100 to 200 diameters. A stroboscope consisting of a 16-inch-diameter, two-slot aluminum disk rotating at any desired speed in the neighborhood of $1800 \mathrm{rpm}$ permits the observer to view the cross-section of the beam at any desired phase, with a resolving time of about $1 / 2 \mathrm{~ms}$.

The monitor has been used only occasionally, and in qualitative manner only. When not in use it is protected by a remotely-controlled, 0.06"-thick, stainless steel diaphragm situated 6" upstream. The minimum distance between mirror (or diaphragm) and axis of the straight section tank is $1.8^{\prime \prime}$. 
14.6

Main References

See memoranda; etc., mentioned above.

Other References

A. Krolzig "Aspects of beam detecting devices," CEA-TM-31, 5/6/58.

K. W. Robinson "Multi-turn beam alignment," CEA-78, 2/15/60.

L. Holcomb, D. I. Porat, K. W. Robinson "CEA system for measurement of electron beam intensity and position," CEA-89, 6/15/62.

L. Holcomb, D. I. Porat, K. W. Robinson "Measuring position and current of an accelerated particle beam," Nuclear Instruments and Methods 24, 399, 1963. 


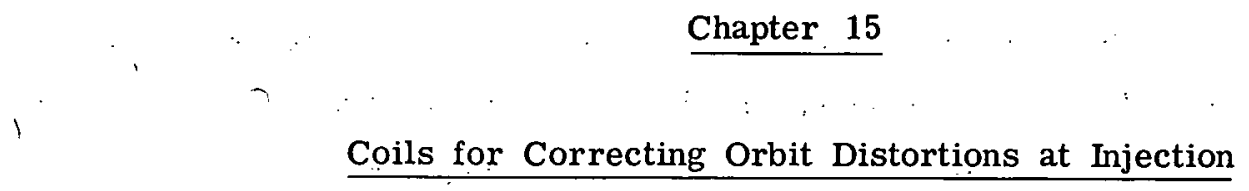

15.1 Introduction

15.2 Correction of horizontal distortions

15.3 Correction of vertical distortions

15.4 Correction coils not now in use

\subsection{Introduction}

At the time of injection, the horizontal and vertical distortions of the equilibrium orbit are large enough to reduce beam intensity considerably. Distortions that are in such relationship as to contribute to the 5th, 6th, and 7th harmonics of orbit shape are especially harmful, for reasons explained in Chap. 5.

If no corrections are made, the distortions at injection are large. The horizontal distortions tend to be especially large - as large as 1 inch, for example, when the magnets are powered for 5 or $6 \mathrm{Bev}$ operation. These distortions are presumed to be due to inequalities in the fields of the 48 magnets; the magnitude of the field at injection is about 35 gauss, and the remanence of the magnet core blocks and end packets is known to vary from magnet to magnet. Perhaps leakage currents play a part also. In addition it is likely that some of the extraneous fields discussed in the following paragraph contribute to the horizontal distortions.

The vertical distortions tend to be smaller, since variations in the (vertical, or near-vertical) fields in the 48 magnets tend to produce horizontal, rather than vertical, distortions. However, various extraneous fields are present also, and their horizontal components may be responsible for the vertical distortions. The earth's magnetic field amounts to about 1 gauss, and the horizontal component in Cambridge, Mass., is about 0.2 gauss. This is large enough to affect the orbit noticeably. The magnetic field due to the Harvard cyclotron is significant also; the cyclotron is only about $35 \mathrm{ft}$. from Straight Sections $3,4,5$, and 6 , and produces fields of the order of 1 gauss here; if the cyclotron magnet is suddenly turned off, there is an immediate change in the orbit distortions.

\subsection{Correction for Horizontal Distortions}

The horizontal distortions at injection are evaluated and then corrected by means of backleg windings on the 24 closed magnets. The individual winding consists of four 
turns, and these embrace the back leg of one half of a closed magnet. As explained in CEAL-TM-128, there are 24 pairs of windings in all; each pair is capable of producing a horizontal "bump" in the neighborhood of one of the closed magnets.

Consider, for example, the pair consisting of a winding on the downstream half of Magnet 3 and the upstream half of Magnet 7. They are about a half a betatron wavelength apart, and Magnet 5 lies midway between them. Accordingly if the operator energizes these windings identically, he produces a localized, horizontal, beam bump the peak of which lies in Magnet 5. The disturbances produced by the two windings almost cancel in all portions of the ring except the short portion between Magnets 3 and 7 .

To appraise the (uncorrected) horizontal distortion at Magnet 5 , the operator varies the sign and magnitude of direct current (of the order of $1 \mathrm{amp}$ ) in the pertinent pair of windings while observing the beam intensity. He soon finds how far the beam can be displaced inward and radially outward before it ceases to lie within the useful region of the magnet-gap cross-section and is lost. If he finds the two extreme displacements to be equal, he concludes that the horizontal distortion normally present here is zero. If he finds them to be unequal, he computes half the difference and regards this as the measure of the distortion. Using the successive pairs of windings, he computes the distortions in each of the 24 magnets.

Knowing these distortions, he can easily establish a sct of 24 potentials which, when applied to the 24 pairs of windings, will produce an orbit that is almost free of horizontal distortions.

The windings, power supplies, and procedures followed are described in CEALTM-128.

The near-elimination of the horizontal distortions has proven to be helpful. Typically, the number of electrons captured into stable orbits is increased by 30 to $100 \%$. Corrections are especially necessary when the synchrotron is operated at energies exceeding 4 Bev.

If the level of synchrotron operation is changed, e.g., from $3 \mathrm{Bev}$ to $5 \mathrm{Bev}$, the distortions change sufficiently so that a different set of correcting currents is required. Some change seems to occur from day to day even when the operating level is held fixed. Changing the injection energy also affects the distortions and necessitates changing the corrections. 
The (modest) correcting fields are of no significance during the latter part of the cycle, when the field strength of the 48 magnets is very large and remanence and extraneous fields are relatively insignificant.

The windings under discussion here have nothing to do with the windings used for beam-bump target engagement described in Chap. 16.

The backleg windings under discussion have a valuable supplementary use: they can be made to produce a bump in the orbit at injection when an experimentalist is using a target situated within the vacuum ring at a location only about 1 inch from the equilibrium orbit of the fully accelerated electrons. Such a target would be badly in the way, at injection, if there were no local bump to make the electrons detour.

\subsection{Correction of Vertical Distortions}

At injection, the vertical distortions tend to be many times smaller than the horizontal distortions, for reasons indicated in Section 15.1. Therefore, despite the small vertical dimension of the vacuum-chamber cross-section, a relatively modest number of vertical correcting coils should be sufficient. In 1960 the designers decided to provide coils at 16 regularly spaced stations around the ring, namely the 16 straight sections occupied by rf cavities. No complications such as targets or valves existed at these locations, and there is special merit: in placing the coils here in that these straight sections have no magnetic shielding.

A correction system that employs only 16 stations is, of course, less than ideal. Since open and closed magnets have different phase-angle length (see Chap. 3), since the two kinds of magnets have differently shaped fringing fields, and since successive rf cavities are not equivalently situated with respect to the two kinds of magnets, better correction could be achieved if, say, 24 stations were used. However, the extent of correction achieved with the present 16 stations appears to be adequate.

At each station, four coils are used. They lie in vertical planes, and two are mounted on each lateral face of the $\mathrm{rf}$ cavity. The two outermost coils are connected in series and called Set A; the two inner ones comprise Set B. The design of the coils is indicated in Drawing E-13M-155 and E-13M-157 and in formal specifications dated June 2, June 7, and June 27, 1961. Atkins \& Merrill, Inc., made the coils in mid-1961. Currents of the order of $50 \mathrm{ma}$ are employed. (The coils are part of a larger set discussed in the following section.) 
To evaluate the vertical distortions normally present at injection, the operator connects the 16 Set A coils so as to form 8 pairs, each capable of producing a localized beam bump. After completing the evaluation by a process similar to that described in the previous section, and after making a crude harmonic analysis with respect to the 5th, 6th, and 7th harmonics, he adjusts the d.c. potentials applied to the 16 Set B coils so as to eliminate, or at least greatly reduce, the distortions. Actually the, 16 different potentials may be governed by either of two groups of potentiometers; one group is adjusted so as to be appropriate when the cyclotron is energized to typical extent, and the other is appropriate to the "cyclotron off" condition. A relay, controlled by an on-or-off signal from the Cyclotron Laboratory, brings the correct set of potentiometers into play. (On one occasion the cyclotron was energized in opposite-from-usual sense, which caused the CEA operator much difficulty.) Once the two groups of potentiometers have been adjusted carefully, they may be left unchanged for days or weeks.

The reduction in vertical distortions has proven to be very valuable. Often the accelerator would run very poorly indeed if no reduction were made.

\subsection{Correction Coils Not Now in Use}

The groups of coils mounted on the rf cavities are more extensive than the previous paragraphs suggest. There are six, not four, vertical coils mounted on each rf cavity, and there is a horizontal coil lying above the cavity and another such coil lying below the cavity. The designers expected that, collectively, these coils would perform three functions: Correcting for horizontal distortions at injection, correcting for vertical distortions at injection, and eliminating coupling between the horizontal and vertical betatron oscillations at injection.

This last function - eliminating coupling - requires comment. When the proton AGS at CERN was started up, considerable "twist" of the quadrupole fields of the magnets was present; this implied that the two principal modes of betatron oscillation were tilted modes. (Ideally, the modes are horizontal and vertical.) This meant that coupling existed between horizontal and vertical oscillations, hence any horizontal oscillation soon became . converted into a vertical oscillation. This was disastrous for a particle undergoing a moderately large horizontal oscillation since the height of the vacuum-chamber cross section is much less than the width; such particles were soon lost from the beam. A highly satisfactory way of eliminating such coupling is to eliminate the twist of quadrupole 
field and also make sure that the frequencies of horizontal and vertical betatron oscillation differ appreciably. By employing suitably designed correction coils the accelerator operator can go a long way toward achieving both these goals. (If much twist were to remain, he could try to eliminate the coupling by making the two frequencies differ by a large amount; but in trying to achieve a large difference he would unavoidably shift each individual frequency by an even larger amount and might then find that, at some moment during the acceleration interval, one of the frequencies became coincident with a stopband and beam blow-up occurred.)

In 1962 the CEA designers found that, fortunately, the CEA ring of 48 magnets produces fields that have no appreciable twist. Perhaps this is because the magnets have such short gaps that no nearby, asymmetrically located, iron object (e.g., the magnet girder) can play an appreciable part in the magnetic circuit. In any event, tests showed that the coupling between the two frequencies was small or non-existent.

Should such coupling ever arise, the uperator could make the necessary corrections by bringing into play the (currently not used) coils referred to above.

Bibliography

H. Mieras, J. M. Paterson, and G. Voss "Correction of radial orbit distortions," CEAL-TM-128, 2/18/64. 
16.1. Internal targets

16.2 Beam-bump method of target engagement

16.3 RF turn-off method of target engagement

\subsection{Inleridal Turgets}

A typical internal target consists of a short straight piece of tungsten ribbon situated inside a straight section tank. The ribbon is mounted transverse to the axis of the tank and is perhaps 0.7 to $1.4^{n}$ from that axis. When the orbiting electrons are caused to strike the ribbon, a slender, forward-traveling bremsstrahlung beam ( $\mathrm{x}$-ray beam resulting from the sudden deceleration of electrons) is produced. Being unaffected by magnetic fields, this beam of high-energy photons proceeds straight ahead and emerges from the synchrotron approximately tangentially via a spout on the downstream end of the following vacuum chamber. Such photon beams have been the mainstay of most of the experiments performed at CEA. (For an account of the production of an external electron beam, see the next chapter.)

Other kinds of external targets have been used. A group of physicists studying the scattering of electrons by protons has employed internal targets of other materials, including carbon, polyethylene, and lithium hydride; also it has used liquid hydrogen confined within a slender container made of mylar film.

The thickness of a typical internal target of metal is usually about $0.1 \mathrm{x}_{\mathrm{o}}$, where $\mathrm{x}_{\mathrm{O}}$ is the "radiation length" of the high-energy electrons in the material in question. Such a target is thick enough to provide an intense beam of photons, yet is thin enough so that the amount of scattering involved is small and the angular width of the bremsstrahlung beam is small also. For tungsten, $\mathrm{X}_{0}$ is $0.13^{\prime \prime}$, and accordingly the typical thickness used is $0.01^{\prime \prime}$.

If the thickness is much greater than $0.1 \mathrm{x}_{\mathrm{o}}$, the amount of scattering is greater, the angular width of the bremsstrahlung beam is increased, and the target may become so hot that provision for cooling must be made. If the thickness is much less, such as $0.01 \mathrm{x}_{\mathrm{o}}$, the intensity of the bremsstrahlung beam is reduced and multiple traversal may occur, that is, many of the electrons striking the target may pass through with such small decrease in energy and such small angular deviation that they may continue in orbit and thus may strike the target again on a subsequent trip around the orbit. Multiple traversal is usually 
welcomed by an experimentalist who is employing a hydrogen target that is small enough so that the region-of-origin of the photon beam remains satisfactorily small (i.e., despite the increased width of the multiple-traversal electron beam). The actual choice of thickness of target, and also the choice of width, depend, of course, on the intensity and geometrical precision that the experimenter requires of the bremsstrahlung beam.

An internal target is usually mounted in a straight section tank, and is thus situated within the vacuum ring. It is mounted in the horizontal median plane of the synchrotron, and is situated about $0.7 "$ to $1.4 "$ to one side of the equilibrium orbit (which coincides, or nearly coincides, with the centerline of the tank). If the target is situated between the accelerator centerpoint and the tank centerline, the target is said to be inside. Otherwise it is said to be outside, although it is, of course, still within the tank. An inside location is usually preferred, since this permits the target to be engaged by the rf turn-off method, discussed in a following section. A target situated in Straight Section 5 must be mounted in an outside location in order that the bremsstrahlung beam will clear the rf cavity in the next straight section downstream. The same is true of a target in Straight Section 11.

The target is usually mounted in such a way that its distance from the tank centerline can be adjusted accurately by remote control. The typical mounting includes a reversible electric motor, a worm and gear reduction system, and a shaft that runs through bellows providing the necessary vacuum-tight seal. The assembly is attached to a port of the straight section tank. Limit switches prevent over-travel, and the exact position of the target is measured with the aid of a linear potentiometer. A special survey fixture that bears against the backs of the two adjacent magnets may be used to calibrate the potentiometer in terms of radial position of the target. The necessary controls, and also a digital voltmeter indicating the potentiometer setting, are situated in the control Ruom. Further details concerning the target mounts are available in B. K. Vetter's memorandum of $6 / 28 / 63$ on "Internal Target Devices."

Typically, targets are maintained ready for immediate use in each of several straight sections. To activate a given bremsstrahlung beam, the operator, by remote control, advances the pertinent target to the desired position close to the beam. It is sometimes the practice to advance a second target - in another straight section - just far enough so that a small fraction of the orbiting electrons will strike this latter target and provide a small but useful flux of photons for use by some parasiting group. Alternatively, two different targets may be engaged fully, sequentially during different pulses of the 
synchrotron, by the beam-bump method, discussed in the following section. This is called time-sharing.

\subsection{Beam-Bump Method of Target Engagement}

The most versatile method of target engagement is the beam-bump method, which entails distorting a portion of the equilibrium orbit.briefly in such manner that the orbiting electrons strike the target even while the rf power level is high and the electrons are still in synchronism with the rf fields. The distortion is appreciable only in one quarter of the ring and is created only during the last few milliseconds of the acceleration interval.

Fig. 16.1 indicates, in exaggerated manner, the form of the distortion employed when the target is situated 1.2" inward from the centerline of Straight Section 8. The distortion is large only between Magnets 2 and 13, and is negligible elsewhere. To create the distortion the operator produces simultaneous changes, or pulses, in the fields of four particular magnets, namely Magnets 2, 6, 9, and 13. Specifically, he produces changes of $-\Delta B,+\Delta B, \frac{1}{2} \Delta B$, and $-\frac{1}{2} \Delta B$ in these respective magnets. The reason for producing "half-size" changes in the latter two magnets is that they are closed magnets and hence have $\beta^{\frac{1}{2}}$ values approximately twice those for open magnets; yet it is desired that the downstream half of the distortion have roughly the same magnitude as the upstream half.

The pulse has the form of half a sine wave. Its duration is about $5 \mathrm{~ms}$ (or, on some occasions, about $11 \mathrm{~ms}$ ) and the timing is such that the peak of the pulse occurs at nearly the same time as the peak of magnet excitation. The magnitude of the pulse is adjusted empirically by the operator; it must be small enough so that the spill-out time will be long and must be large enough so that all of the orbiting electrons will strike the target.

The pulses in the magnetic field are produced by current pulses in special backleg windings on the magnets in question. The winding used on an open magnet has four turns, and that on a closed magnet has two turns; the various turns on any one winding are connected in series, and the windings on successive magnets are likewise connected in series. A happy feature of the scheme is that the voltages induced in the backleg windings in Magnets 2 and 6 are equal and opposite, and the same applies to the voltages induced in the backleg windings on Magnets 9 and 13.

The following somewhat naive argument helps explain the choices of magnets to be bumped and the signs of the bumps. Let us consider the paths of the orbiting electrons at 
an instant when the distortion is large but not large enough to cause the electrons to strike the target. Because the pulse in Magnet 2 is negative, the field in that magnet is reduced and the electrons traveling along there tend to proceed more nearly straight ahead; thus they start traveling along a path that has a radially. outward trend. As they approach Magnet 6 they are traveling in a radially inward direction since the pathlength from Magnet 2 to Magnet 6 is about one half of a betatron oscillation wavelength. The positive pulse in Magnet 6 deflects them more sharply inward, so that, as they enter Straight Section 8, their distance from the old equilibrium orbit is especially large almost large enough to cause them to strike the target situated 1.2 " radially inward there. As they approach Magnet 9 they are traveling rapidly outward and are about.to cross the old equilibrium orbit at a steep angle; but the positive pulse here reduces the rate of outward travel. As they approach Magnet 13 they are traveling inward, and the negative pulse there allows the direction to become almost coincident with the old equilibrium orbit. In summary, the Magnets 2 and 6 produce a large distortion and Magnets 9 and 13 annul it.

At a later instant the current pulse is larger, the distortion is larger, and some of the electrons strike the target; the first to strike are those that have a large amplitude and favorable phase of horizontal betatron oscillation. As the current pulse and the distortion continue to grow, additional electrons strike the target.

'T'o compute the form of the distorted orbil, the designer' takes advantage of a superposition principle: he computes the four individual distortions that would result from pulsing the four magnets singly, then adds the four resulting functions. Throughout most of the magnet ring the sum is small, but in the quadrant under consideration it is far from zero and has its maximum value near Straight Section 8. Actually, the maximum occurs several feet upstream from the midpoint of that straight section.

The heavy curve shown in Fig. 16.1 is, of course, the curve of the new, or distorted, equilibrium orbit. Typical electrons do not travel exactly along this orbit, but execute horizontal and vertical betatron oscillations about it.

If all four pulses are reversed in sign, the direction of the distortion is reversed and the beam can be made to strike a target situated radially outward from the old equilibrium orbit.

Increasing the magnitude of all four pulses in the same proportion increases the magnitude of the bump and permits the operator to place the target farther from the old equilibrium orbit. 
On 3/1/64 the beam-bump target-engagement system was in routine use. Bumps could be produced for serving targets in any of seven straight sections, namely \#4, 5, 7, $8,10,11,14$, and also the middle of Magnet 4 . The necessary backleg windings (on a total of 17 magnets) were installed in Aug. 1963. Preliminary trials were made with the aid of improvised power supplies, while carefully designed power supplies were on order. The current pulses in the backleg windings are produced with the aid of a storage capacitor and a choke coil; the peak current required in the backleg windings when a $1.4^{\mathrm{m}}$ beam bump is to be produced in a 6 -Bev beam is 275 amps. When bumps are to be produced at two different straight sections sequentially, so that time-sharing by two groups of experimentalists can be achieved, two different power supplies are used and a specially designed gating system, described in Chap. 23, is employed.

The beam-bump method works well for all levels of synchrotron operation. The spill-out interval depends not only on the timing and magnitude of the pulse of magnetic. field but also on the half-width of the beam of orbiting electrons and on the rf modulation wave form. The half-width of the beam at the end of the acceleration interval is large when the accelerator is operated at 1 Bev or $6 \mathrm{Bev}$, but is small when it is operated near 3-Bev. (Chap. 3 presents some estimates of the width under various circumstances.) Thus the spill-out interval is comfortably large $(0.5$ to $1.0 \mathrm{~ms})$ at energies near $1 \mathrm{Bev}$ or $6 \mathrm{Bev}$, but may be only about half as great at 3 Bev. However, even during operation at 3 Bevi, spill times as long as 2 (or possibly 3) $\mathrm{ms}$ can be achieved if the length of the beam-bump current pulse is increased to about $11 \mathrm{~ms}$. Such a long (18\%) duty cycle is achieved at the cost of a considerably increased range of output energy.

The beam-bump system is used with enthusiasm by most groups of experimentalists. It is regarded especially highly by experimentalists who employ extremely thin $\left(\sim 0.01 \mathrm{x}_{\mathrm{o}}\right)$ internal targets and wish to make use of multiple traversal. Under favorable circumstances the beam-bump system permits a given orbiting electron to traverse the target as many as 20 or 40 times.

Bibliography

T. L. Collins, informal memorandum of about 6/23/61.

L. A. Law, informal memorandum of $9 / 4 / 63$ on machine signals, beam-bump programming, etc.

R. J. Averill, informal memorandum of $2 / 21 / 64$ on "Beam bump circuits."

R. J. Averill and R. I. Samuel, informal memorandum of $3 / 25 / 64$ on "Instructions to produce beam bumps." 


\subsection{RF Turn-Off Method of Target Engagement}

The simplest method of causing orbiting high-energy electrons to strike an internal target (situated, say, 0.7 to $1.4^{\mathrm{n}}$ radially inward from the centerline of the straight section tank) is to turn off the $\mathrm{rf}$ accelerating power at (or perhaps 100 to $1000 \mu \mathrm{s}$ before) the instant the field strength of the 48 magnets reaches the peak value. The electrons lose energy rapidly by the synchrotron radiation process discussed in Chap. 25, and hence spiral inward and, after several trips around the orbit, strike the target. Electrons of $6 \mathrm{Bev}$ energy spiral inward at the rate of about $0.8 \mathrm{~mm}$ per turn; $3 \mathrm{Bev}$ electrons spiral inward at the rate of $0.1 \mathrm{~mm}$ per turn. The respective spill-out times, or intervals of target engagement, are about $10 \mu \mathrm{s}$ and $20 \mu \mathrm{s}$. When the electron energy is as low as $1 \mathrm{Bev}$, the rate of energy loss by synchrotron radiation is so low that the field strength of the magnets may begin to decrease rapidly before any great loss has occurred and: consequently the electrons may spiral outward instead of inward.

The operator may shorten the spill-out time by using premature rf turn-off, i.e., by turning off the $\mathrm{rf}$ power shortly before the magnet excitation reaches a peak. The electrons then spiral inward "for two reasons." By employing premature rf turn-off the operator may produce fairly satisfactory spill-out even when the synchrotron is adjusted for $1 \mathrm{Bev}$ operation; however, some anomalous beam-loading of the rf cavities may occur and spikes may be found in the curve of photon flux vs. time. (There is some reason to . believe that the beam-loading difficulty, and the spikes, would be reduced if the rf frequency were shifted by, say, $50 \mathrm{kc}$ at this latter part of the cycle.)

Usually the experimentalists want the spill-out time to be long, in order that their detectors will not be saturated. To lengthen the spill-out time the operator modifies the rf modulation wave form by adding a back porch, discussed in Chap. 10. He does this by turning the rf power level down but not off; he reduces it to a critical, intermediate (and gradually decreasing) value such that most of the electrons remain in stable orbits but, in each microsecond, the electrons that have the largest amplitudes of synchrotron oscillation lose synchronism with the rf field, spiral inward due to loss of energy by synchrotron radiation, and strike the target. By adjusting the level and slope of the back porch the operator can provide spill times as long as $1000 \mu \mathrm{s}$, corresponding to a $6 \%$ duty cycle. Of course, when the spill time is as long as this some variation in energy of the impacting electrons occurs, e.g., a $2 \%$ variation. 
Before November 1963 nearly all of the bremsstrahlung beams produced at CEA were produced by the $\mathrm{rf}$ turn-off method and with the use of a suitable back porch. The method worked reasonably well, but has been largely superseded by the far more versatile beam-bump method.

Bibliography

T. L. Collins and J. R. Rees "Trajectories of gamma-ray beams produced by targets within the principal orbit," CEA-A-29 of $10 / 7 / 60$. 


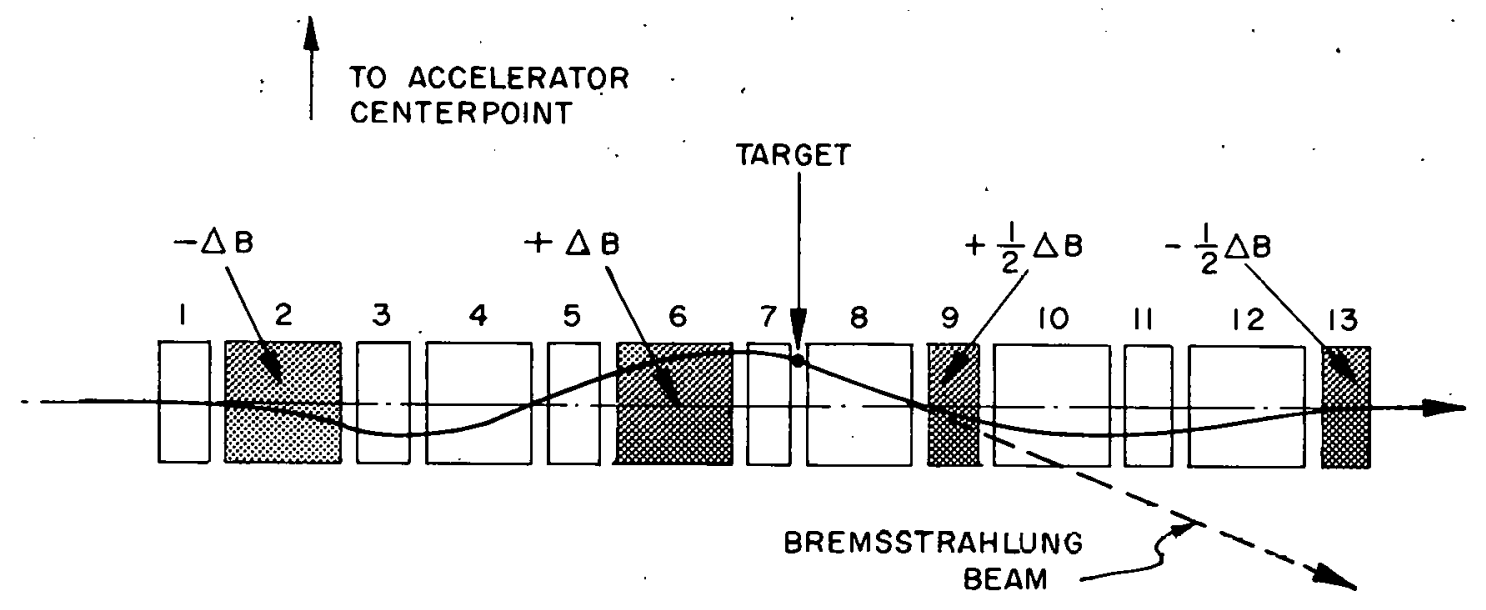

Fig: 16.1. Schematic, plan-view diagram of orbit distortion produced when Magnets 2, 6, 9, and 13 (cross-hatched) are pulsed to cause the electrons to strike a target situated 1.2 inches radially inward in Straight Section 8. The array of magnets has been "straightened out" for ease of exposition, the lengths have been adjusted so as to correspond roughly to phase-angle length with respect to horizontal betatron oscillation, only the magnet poles are shown, and the magnitude of the orbit distortion is exaggerated. Only the orbit segment from Magnet 1 to Magnet 13 is shown. The path of the resulting bremsstrahlung beam is tangent to the distorted equilibrium orbit at the target location. 


\section{Chapter. 17}

External Electron Beam

17.1 Introduction

17.2 Principle of the resonant extraction method

17.3 Design .

17.4 Performance

17.5 Miscellaneous

\subsection{Introduction}

Many kinds of electron scattering experiments become feasible only when highenergy electrons are made available outside the synchrotron. Ideally the external beam should be slender and the duration, or spill time, should be long. In recent years several groups of high-energy physicists (mainly groups concerned with electron scattering) have urged that such a beam be provided at CEA.

To eject electrons from an alternating gradient synchrotron is difficult since the central function of the strong focusing magnet system is to prevent the electrons from straying from the orbit. The Piccioni-type extraction system, used in proton synchrotrons, is not applicable to electron synchrotrons. It employs a thin plate to extract energy from the orbiting particles so that they may spiral inward and encounter a pulsed ejection magnet; but if the particles are electrons, they will emerge from the plate with intolerably large spread of energy. Pulsed electromagnets of more or less conventional type could be used to eject electrons from the orbit, but the spill time would almost certainly be too short and the intensity would be too uneven.

If an external electron beam having good geometry and a long smooth spill is to be achieved, the extraction system used must meet these two seemingly contradictory requirements: (1) the individual electron must be ejected in a single clean operation, i.e., a single pass through an ejection magnet, yet (2) the electrons must be fed to the ejection magnet gradually, e.g., only $1 \%$ or $0.1 \%$ of them per microsecond. In other words, the process of ejecting the individual electron must be unitary and brief, but the process of producing the external beam as a whole must be statistical and prolonged.

Fortunately a method of satisfying these requirements was found: a "resonant extraction method" described in CEAL-1006. 


\subsection{Principle of the Resonant Extraction Method}

The resonant extraction method employs two special electromagnets $E_{1}$ and $E_{2}$ situated in any two straight sections that are available (i.e., not occupied by an rf cavity or other essential equipment) and are situated at either end of an open magnet; the straight sections actually selected are \#4 and \#5. Each of the special magnets is mounted a short distance from the usual equilibrium orbit, and on the inner side, that is, the side toward the accelerator centerpoint.

Magnet $E_{1}$ consists of a single copper sheet, called a current strip. Being located about $1 \frac{1}{4} n$ to the inner side of the usual equilibrium orbit, it does not influence the electrons appreciably until they have been brought close to it. The simplest way of bringing them close is to turn off the rI power, so that they spiral inward as they lose energy by synchrotron radiation. Another way is to create a "beam bump," i.e., a local distortion of the orbit, by means indicated in Chap. 16. Present indications are that the beam-bump method will usually be preferred, since it is applicable to all energies of synchrotron operation, provides a smaller instantaneous range of energy of the emergent beam, and is compatible with fixed positions of Magnets $E_{1}$ and $E_{2}$.

On being energized with a flat-topped pulse near the end of the acceleration interval, Magnet $\mathrm{E}_{1}$ performs two important functions. (1) By a process discussed in a later paragraph it excites the individual orbiting electron into executing a greatly accentuated horizontal betatron oscillation so that, on some later trip around the ring, the electron will "jump the current strip," i.e., pass $\mathrm{E}_{1}$ on the inner side, as indicated in Fig. 17.1. (2) It repels electrons passing on the inner side; it repels them sufficiently strongly so that, on reaching Straight Section 5, they find themselves about 2" radially inward from the usual equilibrium orbit. They then pass alung tlie gap in Magnet $\mathrm{E}_{\mathrm{g}}$; this magnet. produces the large outward deflection that completes the extraction process.

Function 1 is the most crucial and ingenious. Its success stems from the fact that the usual horizontal betatron frequency (6.4 per turn) is close to, and slightly less than, a $\nu_{x}$ value $(6.5$ per turn) that produces horizontal blow-up of the beam. Thus by energizing current strip $\mathrm{E}_{1}$ with a pulse of suitable polarity and magnitude, the operator drives the electrons into the nearest half-integral resonance. During this time the amplitudes of horizontal betatron oscillation increase exponentially. After many turns around the orbit the individual electron comes so close to $E_{1}$ that, two turns later, it may pass it on the other side. The current strip is so thin (about $1 \mathrm{~mm}$ ) that most of the electrons can jump it cleanly. A few (perhaps 20\%) strike it and are lost from the beam. 
As the frequency of the horizontal oscillation- becomes larger, approaching 6.5 , the frequency of the vertical oscillation becomes smaller. However, it becomes only slightly smaller and does not reach any integral or half-integral value; consequently no vertical blow-up occurs and indeed there is no. noteworthy increase in spread in the vertical plane.

Magnet $\mathrm{E}_{2}$, having an iron core and a short and well-defined gap, produces a more intense field than $\mathrm{E}_{1}$ produces. Magnet $\mathrm{E}_{2}$ also is passive at the start of the acceleration interval and is pulsed near the end of this interval; it deflects the pertinent electrons sufficiently vigorously so that they cross the usual equilibrium orbit in Straight Section 6 and emerge from the vacuum ring via a spout on the downstream end of a specially designed vacuum chamber in Magnet 6.

The choice of. straight sections (Nos. 4 and 5) in which to place $E_{1}$ and $E_{2}$ is such that the ejected electrons leave the magnet ring at an open magnet (No. 6), and accordingly they encounter relatively modest fringing fields while emerging.

The first segment of the path of the electrons that have emerged from the ring conforms to the equation: $\mathrm{Y}=-12.375 \mathrm{ft}$. $-0.48421 \mathrm{X}$, where $\mathrm{X}$ and $\mathrm{Y}$ are the floor coordinates expressed in feet.

The electrons travel in an evacuated pipe. It is expected that, typically, the transport system will include two bending magnets and six small quadrupole magnets; these reduce the cross section, eliminate the "momentum vector" of the nominal exit trajectory, and bring the path into coincidence with the axis of the appropriate beampipe through the Main Shielding Wall.

\subsection{Design}

Magnet $E_{1}$ consists of a copper strip that runs parallel to the centerline of the straight section (No. 4) and about $1 \frac{1}{4} "$ from it, as indicated in Fig. 17.2. The strip is $61 \mathrm{~cm}$ long and $1 \mathrm{~mm}$ thick. The height is $5 \mathrm{~cm}$, but slots cut in the upper and lower edges confine the current to a region about $1.5 \mathrm{~cm}$ high. The upper and lower edges of the strip are clamped to water-cooled, pierced stainless steel bars, and are cooled by. them. However, the strip is electrically insulated from them by thin layers of dielectric material. During 6-Bev operation of the synchrotron, Magnet $\mathrm{E}_{1}$ is powered with a flattop, 3150 amp pulse that lasts about $1 \mathrm{~ms}$.

Magnet $\mathrm{E}_{2}$ is more conventional. The $61-\mathrm{cm}$-long core is of laminated construction and is excited by a one-turn winding of water-cooled, pierced copper. Both legs of the 
winding lie entirely within the magnet gap and their exact positions are such that very little field is produced outside the magnet. (Any appreciable external field just in front of the magnet might adversely affect the paths of electrons that have not yet jumped the current strip.) The useful area of the cross section of the magnet gap is $2.5 \mathrm{~cm}$ wide and about $0.8 \mathrm{~cm}$ high. The pole faces are not flat and parallel, but are shaped to produce a small amount of horizontal focusing and vertical defocusing; the strength of the horizontal focusing is such that the width of the emerging beam is a minimum at the exit spout of the vacuum chamber in Magnet 6. Magnet $\mathrm{E}_{2}$ is mounted so that the center of the gap is about $2 "$ from the usual equilibrium orbit. During $6-\mathrm{Bev}$ operation of the synchrotron the magnet is powered with a 5500 amp pulse that produces a field strength of 8500 gauss. The flat-top portion of the pulse lasts about $1 \mathrm{~ms}$. The shape of the pulse may be programmed to compensate for changes in electron energy that occur if $\mathrm{rf}$ turn-off takes place shortly before or shortly after peak excitation of the 48 magnets.

The power supplies for $\mathrm{E}_{1}$ and $\mathrm{E}_{2}$ are of very special type, employing energystoring chokes and transistor-bank switching circuits.

\subsection{Performance}

In May 1963 the resonant extraction method was tried out in abridged manner. Magnet $\mathrm{E}_{1}$ was used, but not Magnet $\mathrm{E}_{2}$; the gual was to show whether the electrons could be diverted successfully to the location that Magnet $\mathrm{E}_{2}$ would uccupy. The trials wcrc highly successful: irrespective of whether the accelerator was operated.at $1 \mathrm{Bev}, 2 \mathrm{Bev}$, or $6 \mathrm{Bev}$, and irrespective of whether the spill time was long or short, the desired diversion of the electrons was accomplished, and the extraction efficiency was about 60 to $70 \%$.

In July 1963 trials were made with $\mathrm{E}_{2}$ also in operation; however, only an improvised, low-power power supply was available and the accelerator energy was held down to 2 Bev. Measurements made on the fully ejected external beam of $2 \mathrm{Bev}$ electrons showed that the beam could be focused so as to have horizontal and vertical widths of about 1.0 and $0.5 \mathrm{~mm}$ respectively, and that the product of linear width and angular width was about $0.1 \mathrm{~cm}$-milliradian and $0.3 \mathrm{~cm}$-milliradian for the vertical and horizontal cross sections respectively. Even smaller values would have been found if no exit window had been used between accelerator and analyzing system, i.e., if no scattering had occurred. Beam optics, extraction efficiency, and spill-out time were found to be in good accord with the theory that has been developed. (See bibliography.) 
In mid-March 1964 the electron beam was ejected successfully during 3-Bev, 5-Bev, and 6-Bev operation of the synchrotron. Specially designed power supplies were used and the engagement of Magnet $E_{1}$ was accomplished both by $\mathrm{rf}$ turn-off and by beam bump. Spill-out intervals as long as $1 \mathrm{~ms}$ were achieved.

\subsection{Miscellaneous}

The external electron beam will probably be ready for routine use by the summer of 1964. The intensity and transverse position of the beam will be monitored with the aid of toroidal ferrite cores. In preliminary tests a Faraday cup has been used to monitor beam intensity.

It is possible that, within about a year, a switching system for directing the external electron beam to either of two beampipes through the Main Shielding Wall will be provided.

Bibliography

F. W. Brasse, G. E. Fischer, M. Fotino, K. W. Robinson "External electron beam of the CEA," CEAL-1006, Sept. 1963. 


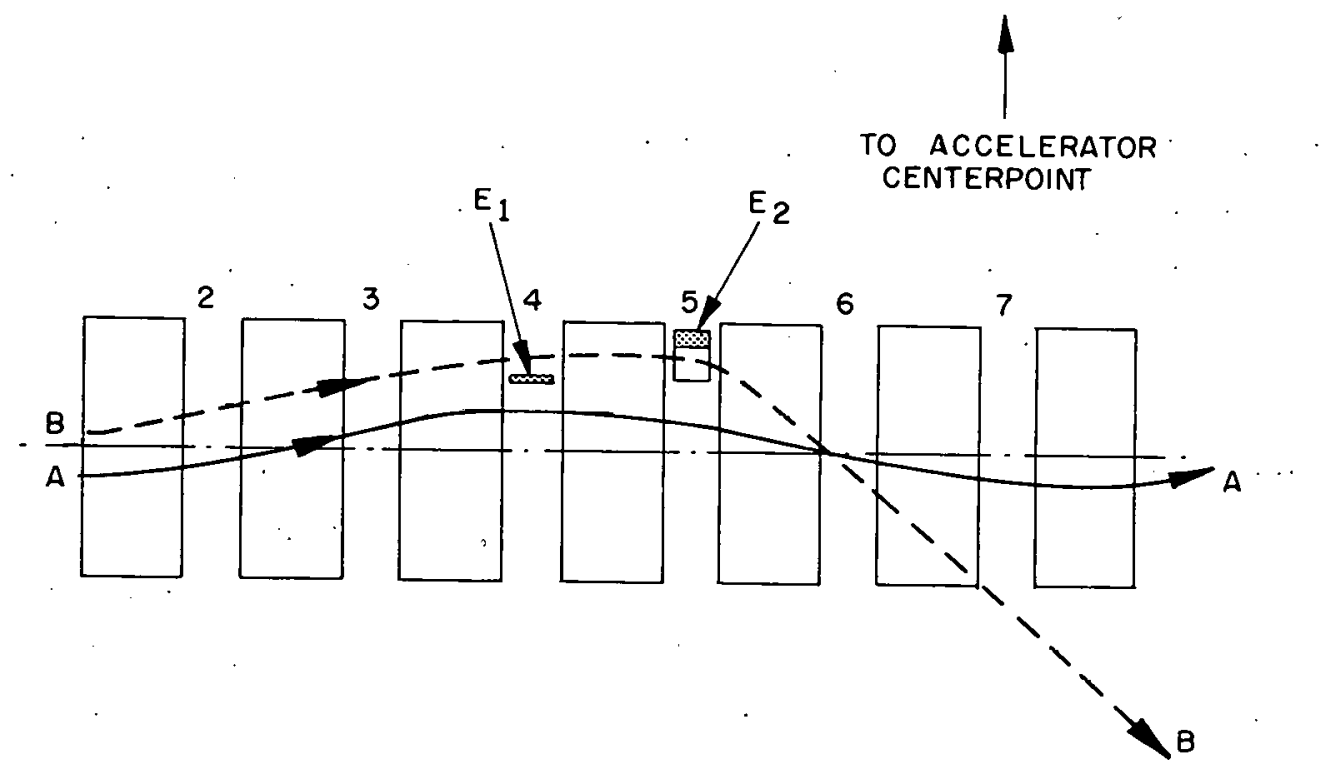

Fig. 17.1. Schematic plan view of the path of an electron during ejection by the resonant extraction method. Seven magnets, shown here with greatly exaggerated radial dimension, are included, and the serial numbers of the straight sections are indicated. Curve $A$ is the path of an electron executing a typical horizontal betatron oscillation about the usual equilibrium orbit. Curve $B$ is the path that pertains after Magnet $E_{\text {, (the current }}$ strip) has been energized, the rf power has been turned off, and the electron has "jumped the current strip;" the electron is deflected toward Magnet $E_{2}$, which deflects it sharply outward.

a.

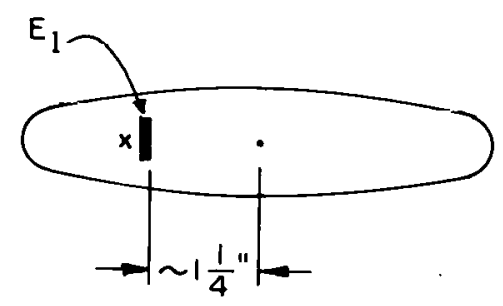

— TO ACCELERATOR CENTERPOINT

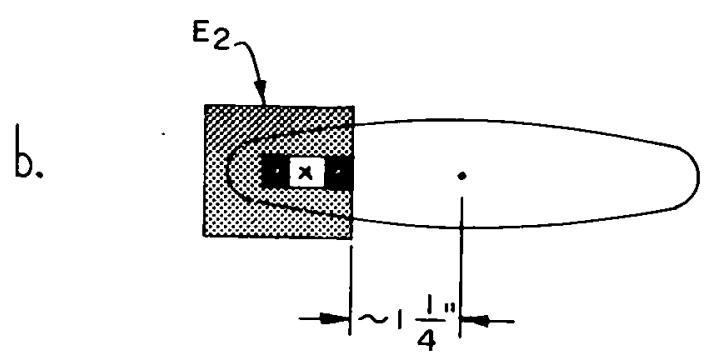

Fig. 17.2. Schematic diagrams of cross-sections of (a) Magnet $E_{1}$ (current strip) and (b) Magnet $E_{2}$, as seen by an observer looking downstream. The large oval represents the cross-section of an adjacent vacuum chamber, and the small " $x$ " indicates the path of an electron that jumps the current strip $E_{1}$ and later enters the gap of $E_{2}$. 


\title{
Cabling and Wiring
}

\author{
18.1 Introduction \\ 18.2 Cable from Cambridge Electric Light Co. \\ 18.3 Limitations on power available to CEA \\ 18.4 Cubicle system for $13.8 \mathrm{kv}$ power \\ 18.5 Electrical grounding system of the CEA \\ 18.6 Power factor correction system
}

\subsection{Introduction}

Cables and wires are used for an impressive variety of purposes, most of which may be classified as transmission of power, control of power, or transmission of signals. They serve not only the accelerator proper but also the major equipments used by experimentalists.

The major applications of electric power are those concerned with powering the 48 magnets of the accelerator', powering the transmitter supplying $\mathrm{rf}$ power to the $16 \mathrm{rf}$ cavities, and powering the various special magnets used by experimentalists. Transmission of signals includes signals for monitoring the performance of the accelerator, signals for controlling the accelerator, and the enormous volume of signals transmitted from the experimentalists' detectors to the Counting Room.

The CEA Electrical Engineering Division (see Chap. 2) is responsible for the planning, execution, and maintenance of all major cabling systems at CEA. It selects the type of cable, plans the routing, installs the cable, and tests it. In addition it decides what kinds of cable to regard as standard, maintains adequate inventories of each type, and establishes color coding conventions. It prepares and files drawings showing the cable layouts for each major application. It carries out any major changeovers required when one experiment is completed and another is to begin: It designs, installs, and maintains interlock systems for the synchrotron area and other high-radiation areas, and provides the associated command lights, information lights, warning beacons, warning beepers, crash buttons, etc. It provides equipment for monitoring the total power and total MVA figure drawn from the Cambridge Electric Light Co., and helps see that consumption does not exceed the established ceilings. It provides the necessary safeguards for electrical safety. 
An individual cabling job may entail, in addition to choosing the type of cable, performing the following tasks:

Routing: Finding the most suitable locations for the cable runs. Often, formal routing diagrams must be prepared in advance.

Providing cable trays: Many cables rest in trays mounted on walls. The tray systems must be designed, procured, and installed before the cables are pulled into place.

Cable pulling: Large number's of cables run via trays. Others run via conduits in the concrete floor or conduits in thick concrete walls. Elimination of obstructions in the conduits is sometimes a difficult lask. Pulling the heavy, stiff cables through the conduits is difficult also. Drawings showing the locations and sizes of the conduits are available.

Attaching connectors and terminals: Much effort is given to procuring connectors and terminals that permit quick changeover from one, array of experimental equipment to another as successive groups of experimentalists are granted priority of use of the synchrotron.

Verifying the correctness of hook-up: Newly installed cables cannot be put to routine use until they have been tested.

Labeling: To minimize the number of cables that no longer have any useful purpose and merely preempt valuable conduit space, the Electrical Engineering Division provides several identifying bands on each cable and makes occasional checks to see which cables, if any, are no longer needed.

Posting of warning signs, etc.; Terminals that may: be at high potential are usually enclosed in protective housings, and in addition suitable warning signs, warning lights (yellow, ordinarily) are mounted nearby.

. Controlling the electrical length of a cable: Cables used for transmitting data with accurately known time delay must be cut so as to have the correct electrical length. In some instances errors as small as $3 \mathrm{ft}$. may be harmful.

In choosing the type of copper cable appropriate to a given application, the Electrical Engineering Division engineers consider many factors, including the current and voltage ratings, number of conductors required per cable, resistance per foot, expected temperature rise, flexibility, color of outer. surface and compatibility with color-coding conventions, possible meed for external electrostatic shielding, vulnerability of the insulation to heat, ozone and nuclear radiations. Price, delivery time, and availability of suitable terminals and connectors are considered also. 
Coaxial cables must have appropriate impedance, low attenuation per foot for the frequencies concerned, adequate flexibility and mechanical strength. Price, delivery time, and availability of suitable terminals and connectors are important considerations also. In some instances the exact length ("timing") is important also.

18.2 Cable from Cambridge Electric Light Co.

The primary electrical power received at CEA is three-phase, 60-cycle, $13.8 \mathrm{kv}$ current transmitted from the Cambridge Electric Light Co. via a special, three-conductor cable rated at $15 \mathrm{kv}$ and $9 \mathrm{MVA}$, and designated \#13-34. The cable terminates in CEA Cubicle \#1, discussed in a later paragraph.

\subsection{Limitations on Power Available at CEA}

The present (March 1, 1964) contract with the Cambridge Electric Light Co. requires that, on regular week-day working days, the CEA demand remain below 5 MVA in the 16hour period following 7:00 a.m. At other times the requirement is that the demand remain below 8 MVA; this applies to the 8-hour period starting at 11:00 p.m. on every day and applies throughout all 24 hours of Saturdays, Sundays, and commercial holidays. (It is likely that, at some future time, the CEA will find it necessary to raise the MVA ceiling and will negotiate a new maximum value with the supplier.)

A special telephone line from the CEA Control Room to the Cambridge Electric Light Co. permits CEA engineers to discuss promptly with the supplier any noteworthy change planned in the demand.

\subsection{Cubicle System for $13.8 \mathrm{kv}$ Power}

The $13.8 \mathrm{kv}$ power reaching CEA Cubicle \#1 is distributed via bus bars to 13 adjacent cubicles. Each of these has its own self-protecting circuit-breakers, which also can be operated by electrical means locally or remotely.

Cubicle \#2 contains kilovolt-ampere and kilowatt-hour monitoring equipment owned and serviced by the Cambridge Electric Light Co. Cubicle \#3 contains corresponding monitoring equipment owned and serviced by CEA. Cubicles \#4 and \#5 supply transformers associated with Power. Supplies 1 and 2 for the 48 magnets. Cubicles \#6, \#7, \#12, \#13, \#14 supply transformers serving $500 \mathrm{kw}$ rectifiers for magnets used by experimentalists. Cubicle \#8 supplies transformers serving smaller power supplies and also serving the 
Cryogenics Room and Laboratory Building. Cubicle \#9 supplies transformers serving the Experimental Hall. Cubicle \#10 supplies power serving the linac, rf system, and the Power Bldg. Cubicle \#11 supplies transformers serving the smallest categories of power supplies for magnets used by experimentalists.

\subsection{Electrical Grounding System of the CEA}

The CEA electrical grounding system includes more than one hundred 8-ft-long copper-coated, 3/4-inch-diameter steel rods that have been driven into the ground to depths of 10 to $20 \mathrm{ft}$. below existing grade, at locations near the north, east, and south sides of the Power Bldg., the outer wall of the Experimental Hall, and the Lab. Bldg. The rods are extensively interconnected by a grid of heavy, bare, stranded copper conductors. Similar conductors are used to connect major electrical installations to the grid. A grounding ring of such conductor is provided in the circular tunnel, to permit grounding the magnet girders. Every utility outlet includes a ground wire, connected to the grid.

Certain special grounding cables are insulated, and the usual custom of using greencolored cables is observed.

On some occasions large currents flow in the grounding cables. However, no routine measurements of such currents are made, and indeed the complexity of the grid is such that, in general, meaningful measurements are difficult to devise.

\subsection{Power Factor Correction Equipment}

In 1962 and 1963 the power factor of major CEA installations - especially the power supplies for large magnets used by experimentalists - was low. Consequently the established ceilings on total MVA were unnecessarily restrictivè.

Equipment for correcting the power factor is now being procured. It will consist, for the most part, of high-voltage capacitor banks.

References:

Electrical Engineering Division: "Electrical engineering standards," 11/1/62. 31 pages. 
19.1 Old Control Room

19.2 New Control Room

\subsection{Old Control Room}

Until July 14, 1963, the main controls for the accelerator were centered in Room 103 of the Laboratory Building, a room that was very close to the old Counting Room (Room 104) and hence permitted close working rclationship between machine operators and the experimentalists. It soon became clear, however, that such a close relationship was not essential. When it became clear also that the size of Room 103 was inadequate and that important economies would result if the controls were moved to a site nearer to the control areas for linac, magnet power, and rf power, the decișion was made to move the main controls to the Power Building - and at the same time to mount them in more efficient and more permanent manner.

The move was completed on Aug. 26, 1963, when the synchrotron was operated for the first time after the six-week mid-summer shutdown.

\subsection{New Control Room}

The New Control Room, about $15 \times 50 \mathrm{ft}$. in area, is situated in the west side of the Power Bldg. first floor, and is thus near the various component control areas and the new Counting Room. It is insulated thermally and acoustically from the rest of the building, and is air conditioned. Special slots in the floor provide access for a large number of cables running from the basement. Long glass windows permit visitors to observe operation without entering. At the south end of the room there is a small conference area used by the Operations Division.

The Control Room contains two arrays of racks. The main array, containing Racks 1 through 10 inclusive, is at the north end of the room; it contains all the controls that must be adjusted frequently and all principal indicators. The supplementary array contains Racks 11 through 21 inclusive, which house less-frequently used controls, less essential indicators, and various small power supplies. 
The individual rack is an Amco Engineering Co. Type FX-78-25 unit, 19 inches wide and $7 \mathrm{ft}$. high.

The equipment included in the racks is listcd below. The symbol I\&C means indicators and controls.

\section{Main Array of Racks}

Rack 1

Controls for correcting horizontal orbit distortions

Rack 2 Target position, I\&C

Scraper position indicators

Beampath shutters, I\&C

Interlock system for Synchrotron Area, I\&C

Interlock system for Experimental Hall area, I\&C

Rack $3 \quad$ Linac, I\&C

Inflector, I\&C

Display of indicator lights for interlocked doors

Racks 4 \& 5 Nearly all of the most frequently used controls, including those governing the injection system, rf system, quadrupole-type pole face windings, and the beam-bump system

Vacuum monitoring system meters

Audio communication system, I\&C

Loudspeaker

Alarm and enunciation system

TV monitor tubes

Three multi-trace Type 555 Tektronix CRO's

Patch cables for connecting CRO's to monitors of beam intensity, horizontal displacement, and vertical displacement in the synchrotron ring

Rack 6 Current in the 48 magnets, I\&C

Capacitor switching panel for adjusting frequency of the magnet excitation

Vacuum pump warning lights

CRO display of rf system pārameter's

Rack $7 \quad$ TV camera tubes, remote controls

Digital meter indicating time-integrated intensity of beam in synchrotron

Beam-bump system, I\&C

Rack 8 Terminal board for signals from all monitors of beam intensity, horizontal displacement, and vertical displacement in the synchrotron ring

Terminals for beam spill signal

Terminals for linac signals

Terminals for peaking strip signals

Equipment for exciting betatron oscillations

Rack 9 Equipment for monitoring total power and KVA value for CEA as a whole

Rack 10 Spare

Rack 11 Master oscillator $(26 \mathrm{Mc} / \mathrm{sec}$, for $\mathrm{rf}$ system and linac)

RF system, I\&C

Vacuum Chamber \#48, temperature indicator 
Rack $12 \quad \mathrm{RF}$ system indicators

Spill counter, high voltage supply and distribution system

Rack $13 \quad$ Peaking strip bias supply

Double peaking strip, or predicter, system

Logic circuitry for programming output beams

Beam-bump delay units

Racks 14, 15, Radiation monitoring equipment, I\&C and 16

Rack 17 Coils for correcting orbit distortions at injection, I\&C

Rack 18 Quadrupole and sextupole pole face windings, I\&C

Rack 19. Spare

Racks 20 External electron beam, I\&C

and 21 


\section{Chapter 20}

\section{Shielding}

\subsection{Purpose}

20.2 Main shielding installations

20.3 Inventory of shielding blocks

20.4 Specifications of ilmenite-loaded concrete.

20.5 Effectiveness of ilmenite-loaded concrete in attenuating radiation

\subsection{Purpose}

The main purpose of the shielding used at CEA is to reduce the radiation levels in CEA work areas and other nearby areas to the lowest practical levels (and well below the statutory levels specified in Chap. 21). Another purpose is to reduce the background radiation that strikes detectors in use at CEA or strikes detectors used in other nearby laboratories, e.g., the Biological Laboratories.

The shielding must be effective against all kinds of radiation, including (a) electrons and photons from the synchrotron proper and (b) neutrons, muons, and other secondaries produced when collimators, targets, quantameters, or the like are struck by a beam.

\subsection{Main Shielding Installations}

Since the synchrotron unit is about $10 \mathrm{ft}$. below ground level and much of the radiation propagates approximately horizontally, a large fraction of the radiation from the synchrotron is absorbed in the concrete walls of the circular tunnel and in the surrounding earth.

Radiation levels in the Target Area tend to exceed those in typical portions of the circular tunnel, and accordingly an especially thick and dense shield has been provided above the Target Area. This shield is described in Chap. 23, which deals •with the New Counting. Room.

Between the Target Area and the Experimental Hall there is a Main Shielding Wall consisting of two layers of 4-ft-thick ilmenite-loaded concrete blocks described in detail in a following section. More than 100 Type A blocks, each $4^{\prime} \times 4^{\prime} \times 8^{\prime}$, are included. The blocks are offset, or staggered, vertically and horizontally so that no single crack extends more than half-way through the wall. The wall is in three segments, each about $50 \mathrm{ft}$. long. Each is provided with two channels, or beam pipes, for admitting collimated beams 
to the Experimental Hall. The beam pipes proper are incorporated in blocks of types L, $M, N$, and $\mathrm{W}$, and make an acute angle with the wall. When not in use, the pipes can be blocked with steel stoppers; remotely controlled lead shutters, described in Chap. 30, are provided also. The overall thickness of the wall is $8 \mathrm{ft}$., and the typical pathlength in the wall (parallel to the beam pipe) is about $16 \mathrm{ft}$.

A labyrinth provides a passageway between the Target Area and the Experimental Hall. A broader passageway can be provided by removing certain extra-large (Type D and Type E) door blocks of the Main Shielding Wall.

Each of the major experimental areas in the Experimental Hall is enclosed in a wall of concrete blocks $2 \mathrm{ft}$. or more in thickness. Most of these are $2^{\prime} \times 8^{\prime} \times 8^{\prime}$ FF blocks.

An earth bank extending around the outer wall of the Experimental Hall attenuates radiation lraveling horizontally toward the adjacent parking lot or traveling toward the buildings beyond.

Small shielding walls, or baffles, have been built in Radial Tunnels 2, 14, and 26. These baffles, built with $6^{\prime \prime} \times 12^{\prime \prime} \times 12^{\prime \prime}$ blocks of ordinary concrete and with lead bricks, stop nearly all the radiation traveling along the tunnels but still allow room for persons to walk by.

In December 1962 and July 1963 additional shielding was provided near the northwest edge of CEA property by Hammond St. in order to reduce the radiation levels found there. The radiation originates at a scraper in Straight Section 32 - when the scraper is in use. In August 1963 the temporary shielding used near Hammond St. was replaced by additional earth fill and a concrete wall.

The roof of the Experimental Hall is of light construction. Thus downward scattering of radiation by the roof is minimized.

The skyshine, i.e., radiation scattered downward into work areas by the roof or by the air above the roof, consists mainly of 0 to $5 \mathrm{Mev}$ neutrons and photons. These can be stopped by relatively thin shields. To date, the skyshine has been negligible.

The typical, high-energy photon beam terminates in a quantameter (see Chap. 30) situated at the center of a beam-catcher hut. The sides of the hut are of ilmenite-loaded concrete blocks and are 4 to $6 \mathrm{ft}$. thick; the rear wall is 6 to $8 \mathrm{ft}$. thick; the top is usually $4 \mathrm{ft}$. thick. 


\subsection{Inventory of Shielding Blocks}

Table 20.1 shows the types and quantities of large shielding blocks available for use at CEA. Most of the blocks are of ilmenite-loaded concrete, discussed in the following section.

Ncarly all of the blocks have, at the :top; a recessed lifting lug or a threaded socket in which' an eye-bolt can be inserted. The edges of the larger blocks are protected 'by angle-irons. The dimensions of the blocks are accurate to within $1 / 8^{n}$.

There are several hundred. $6 " \times 12 " \times 12^{\prime \prime}$ blocks of ordinary concrete, several thousand $2^{\prime \prime} \times 4^{\prime \prime} \times 8^{\prime \prime}$ bricks of lead, and a somewhat smaller number of iron bricks of various sizes.

Table 20.1

Inventory of Large Shielding Blocks

(Note: The blocks are of ilmenite-loaded concrete except where indicated otherwise.)

\begin{tabular}{|c|c|c|c|c|c|}
\hline Type & $\begin{array}{c}\text { Dimensions } \\
(\mathrm{H} \times \mathrm{W} \times \mathrm{L}) \\
\text { in inches }\end{array}$ & $\begin{array}{l}\text { Number } \\
\text { on Hand. }\end{array}$ & Type & $\begin{array}{c}\text { Dimensions } \\
(\mathrm{H} \times \mathrm{W} \times \mathrm{L}) \\
\text { in inches }\end{array}$ & $\begin{array}{l}\text { Number } \\
\text { on Hand }\end{array}$ \\
\hline A & $48 \times .48 \times 96$ & 111 & $\mathrm{~T}$ & $22 \times 48 \times 24$ & 5 \\
\hline $\mathrm{B}$ & $144 \times 48 \times 72$ & 3 & $\mathrm{U}$ & $22 \times 48 \times 48$ & 3. \\
\hline $\mathrm{C}$ & $120 \times 48 \times 96$ & 4 & $\mathrm{~V}$ & $22 \times 48 \times 96$ & 4 \\
\hline$D^{*}$ & $192 \times 24 \times 48$ & 36 & $W^{* *}$ & $24 \times 48 \times 114$ & 3 \\
\hline$E^{*}$ & $96 \times 24 \times 48$ & 8 & $\mathrm{X}$ & $34 \frac{1}{4} \times 18 \times 36$ & 42 \\
\hline $\mathbf{F}$ & $48 \times 24 \times 24$ & 16 & $\mathbf{Y}$ & $22 \times 48 \times 18$ & 12 \\
\hline G & $96 \times 24 \times 48$ & 6 & $\mathrm{Z}$ & $24 \times 48 \times 18$ & 10 \\
\hline $\mathrm{H}$ & $72 \times 48 \times 96$ & 21 & AA & $24 \times 48 \times 144$ & 25 \\
\hline $\mathrm{J}$ & $48 \times$ special & 10 & $\mathrm{BB}$ & $120 \times 48 \times 60$ & 2 . \\
\hline $\mathbf{K}$ & $144 \times$ special & 1 & $\mathrm{CC}$ & $24 \times 24 \times 96$ & 8 \\
\hline $\mathrm{L} * *$ & $22 \times 48 \times 96$ & 3 & DD & $24 \times 24 \times 192$ & 48 \\
\hline$M * *$ & $24 \times 48 \times 96$ & 3 & $\mathrm{EE} * * *$ & $24 \times 24 \times .192$ & 16 \\
\hline $\mathrm{N} * *$ & $22 \times 48 \times 114$ & 3 & $F F$ & $96 \times 24 \times 96$ & 48 \\
\hline $\mathrm{O}:$ & $24 \times 48 \times 12$ & 41 & GG & $24 \times$ special & 3 \\
\hline $\mathrm{P}$ & $24 \times 48 \times 24$ & 29 & $\mathrm{HH}$ & $120 \times$ special & 1 \\
\hline $\mathbf{Q}$ & $24 \times 48 \times 48$ & $1 \cdots$ & JJ & $24 \times$ special & 2 \\
\hline $\mathbf{R}$ & $24 \times 48 \times 96$ & 30 & $\mathbf{K K}$ & $96 \times 24 \times 48$ & 32 \\
\hline $\mathbf{S}$ & $22 \times 48 \times 12$ & 5 & LL*** & $24 \times 24 \times 96$ & 8 \\
\hline
\end{tabular}

* These blocks are of ordinary concrete.

** These blocks have central beam pipes of simple type.

*** These blocks have central beam pipes that can be joined (by vacuum-tight couplings) to drift tubes to form evacuated and shielded beam runs. 


\subsection{Specifications of Ilmenite-Loaded Concrete}

The standard shielding-block material used at CEA is ilmenite-loaded concrete, i.e., concrete that contains a high percentage of the iron ore ilmenite. The ore has the formula $\mathrm{FeOTiO}_{2}$ and is about $38 \%$ iron (by weight); it is chemically suitable as an ingredient of concrete and is virtually non-magnetic.

The mixture used in making this concrete contains specified proportions of coarse, medium, and fine grades of ilmenite and a specified amount of water. The density of the finished concrete block is about $240 \mathrm{lb} / \mathrm{ft}^{3}$, or about $3.9 \mathrm{~g} / \mathrm{cm}^{3}$.

Shielding blocks of ilmenite-loaded concrete have. high density and reasonably low cost, and provide high attenuation for a wide variety of radiation types and energies. The iron content is effective at attenuating gamma radiation and most other kinds of radiation, and the hydrogen content performs the essential role of moderating neutrons that havc energy less than a few Mev.

\subsection{Effectiveness of Ilmenite-Loaded Concrete in Attenuating, Radiation}

A. Bremsstrahlung Produced by 4-Bev Electrons. The effectiveness of ilmeniteloaded concrete in attenuating forward-traveling (longitudinal) bremsstrahlung was determined in tests made in 1962 and reported in CEAL-1004. A circulating beam of $4 \mathrm{Bev}$ electrons struck a thin (0.01") tungsten target mounted in Straight Section 8. The resulting bremsstrahlung (photon) beam emerged from the synchrotron via the spout of an exit chamber and passed along an evacuated pipe, through the gap of a clearing magnet, through a hole in the Main Shielding Wall, and into the Experimental Hall. It then struck a variable-thickness shielding wall (of ilmenite-loaded concrete) along the normal. The intensity of radiation emerging in forward $\left(0^{\circ}\right)$ direction was measured by a 20-liter, methane-filled, tissue-equivalent, ionization chamber situated at a fixed location stratght downstream from the wall. The chamber current was determined for various wall thicknesses. Table 20.2 presents the results.

B. High-Energy Neutrons. The effectiveness of ilmenite-loaded concrete in attenuating a transverse beam of high-energy neutrons was determined in tests made in 1963 and reported in CEAL-1007. A bremsstrahlung beam comprising $2 \times 10^{10}$ equivalent quanta per second originated in a thin tungsten target struck by a circulating beam of $5 \mathrm{Bev}$ electrons. After traveling along a 165 -ft-long evacuated pipe the bremsstrahlung beam struck a Wilson-type quantameter situated in a $2^{\prime} \times 2^{\prime} \times 8^{\prime}$ space within a concrete 
Table 20.2

Results* of Experiment on Longitudinal Shielding of Bremsstrahlung Beam Having an End-Point Energy of $4 \mathrm{Bev}$

\begin{tabular}{|c|c|c|}
\hline $\begin{array}{l}\text { Thickness } \\
\text { of wall (ft) }\end{array}$ & $\begin{array}{l}\text { Chamber current } \\
\text { (arbitrary units) }\end{array}$ & $\begin{array}{c}\text { Principal component } \\
\text { transmitted }\end{array}$ \\
\hline $\begin{array}{l}2 \\
4\end{array}$ & $\left.\begin{array}{r}100,000 \\
5,600\end{array}\right\}$ & photons and electrons \\
\hline $\begin{array}{l}6 \\
8\end{array}$ & $\left.\begin{array}{r}36 \\
7\end{array}\right\}$ & neutrons \\
\hline $\begin{array}{l}10 \\
12\end{array}$ & $\left.\begin{array}{l}4 \\
2\end{array}\right\}$ & muons \\
\hline
\end{tabular}

* Data from CEAL-1004

hut. The aggregate thickness of the copper plates in the quantameter was $18 \mathrm{~cm}$ (corresponding to $12.5 \mathrm{X}_{\mathrm{o}}$ ), and accordingly the quantameter acted as a very thick target. The time-integrated amount of neutron radiation emerging from the quantameter at $90^{\circ}$ to the incident beam and passing through a variable-thickness shielding wall of ilmenite-loaded concrete was measured by two different Hankins-type fast-neutron monitors. As indicated in Chap. 21, each of these has an output that is approximately proportional to the biological dose delivered by the neutron flux, irrespective of neutron energy; that is, the reading is in terms of rems, not rads. Each monitor includes also a discriminator that practically eliminates response to gamma radiation.

The half-value-thickness of the ilmenite-loaded concrete for the neutron flux was found to be 3.2" for wall thicknesses of 1 to $2 \mathrm{ft}$. and was $6.4^{\mathrm{N}}$ for thicknesses of 2 to $3 \mathrm{ft}$. The corresponding absorption mean free paths are $4.6^{\prime \prime}$ and $9.3^{n}$.

\section{References}

M. S. Livingston "Study of accelerator grade level," CAP-22 of 2/28/56.

R. W. Williams "Shielding design of the CEA," CEA-10 of 8/31/56.

M. S. Livingston "Radiation shielding for the CEA," CEA-27 of 5/2/57.

M. S. Livingston "Memorandum on radiation shielding for the CEA," CEA-TM-8 of 5/22/57. R. Wilson "Revision of shielding calculations," CEA-73 of 5/12/59.

J K. dePagter and M. Fotino "Shielding measurements on a 4-Gev photon beam," CEAL1004 of $6 / 6 / 63$.

S. Kao and G. A. Voss "Effectiveness of ilmenite-loaded concrete in attenuating neutron radiation produced by a $5-\mathrm{Bev}$ photon beam," CEAL-1007 of 12/20/63. 
Chapter 21

Chapter 21
Radiation Monitoring Equipment
21.1 Introduction
21.3 System of ionization chambers at fixed locations
21.4 Biological dose meters responsive to 0-to-15 Mev neutrons
21.5 Ekco-type portable survey meters
21.6 Film badges for persons
21.7 Film packets used as area monitors
21.8 Other RADSAF. equipment
21.9 Success of program

21.1 Intruduction

The CEA Radiation Safety Officer is responsible for seeing that the CEA RADSAF equipment is adequate and that satisfactory procedures for using it have been worked out. Also, he maintains records of the exposure levels found at various indoor and outdoor stations and of the doses received hy persons. The Operations Division is responsible for the day-to-day use of the equipment and for seeing that the accepted procedures are followed. The procedures are reviewed from time to time by representatives of the M.I.T. Health Physics Division and the Harvard Radiological Services.

The CEA RADSAF program must satisfy the requirements established by M.I.T., Harvard, the Department of Public Health of the Commonwealth of Massachusetts, and the Atomic Energy Commission. Also, it must be conducive to good relations with persons living nearby and with workers in nearby laboratories.

A major goal is to see that no person receives from the CEA a radiation dose in excess of the established limits. For the majority of adult radiation workers in Massachusetts the Massachusetts Department of Public Health has set a limit of 3 rems per quarter year and 5 rems per year. For a non-radiation-worker the rules state that the biological dose he may be "... reasonably estimated to have gotten" must not exceed 0.5 rem per year. (This may be compared with the figure of 0.1 rem per year corresponding roughly to the dose delivered at sea level in Cambridge, Mass., by cosmic rays and radiation from the earth.) The permissible dose for the hands of a radiation worker is about 6 times the permissible dose for his body as a whole. The permissible dose of fast ( 0.5 to $5.0 \mathrm{Mev})$ neutrons is usually stated as 20 per $\left(\mathrm{cm}^{2} \mathrm{sec}\right)$ for a radiation worker and 2 per $\left(\mathrm{cm}^{2} \mathrm{sec}\right)$ 
for a non-radiation-worker; if the exposure dose is measured in terms of rads, the rem values are obtained by multiplying the rad figure by the appropriate RBE value, which lies between 7 and 10 for neutron energies of 0.5 to $5.0 \mathrm{Mev}$, according to NBS Handbook 75 of 1961.

Experiments involving delicate measurements of radiation are in progress in the nearby Biological Laboratory, and the radiation originating at CEA must be attenuated so as not to interfere with those experiments.

\subsection{System of Ionization Chambers at Fixed Locations}

On 3/1/64 this system included eleven regularly active chambers and nine spares that were put to use occasionally. Six of the regularly active chambers were mounted outdoors - two at the perimeter of the northwest parking lot and four just outside the Experimental Hall. Three of the chambers were situated in the Power Building basement (near Tunnels 2, 14, and 26) and one was mounted near the Linac Control Room.

The design of the individual chamber is indicated in Drawing D-251-A. The heart of the device is an $8 \frac{1}{2}$-inch I.D. cylinder made of $1 / 32^{n}$-thick aluminum and lined with a $1 / 8^{\prime}$ layer of epoxy resin. The cylinder has a net volume of 20 liters and contains methane gas at two atmospheres pressure absolute, i.e., contains about 31 grams of methane. Sur-. rounding the cylinder is a cylindrical stainless steel housing having a wall thickness of $1 / 16^{\mathrm{n}}$, and the whole is usually protected by a rectangular aluminum box having a wall thickness of $1 / 16^{\prime \prime}$. Along the axis of the system there is a $1 / 4^{\text {" }}$ diameter stainless steel rod serving as anode. The rod is kept at about ground potential, and the aluminum chamber is kept at about -250 volts. A small air-tight chamber, mounted externally at the top, contains a preamplifier that includes a resistor, an electrometer tube, a magnetic reed relay, and various other electrical parts. Ordinarlly a $10^{13} \mathrm{ohm}$ resistor is used; this provides a useful range of 0 to $1 \mathrm{mr} / \mathrm{hr}$ and a time constant of 200 seconds. If a $10^{12}$ ohm resistor is used, the useful range is 0 to $10 \mathrm{mr} / \mathrm{hr}$ and the time constant is 20 sec. The electrometer tube is used in a cathode follower circuit. The output is fed by shielded cable and Amphenol connectors to the Control Room.

Each ionization chamber is served by a separate "helipot box" situated in a rack in the Control Room, and for every group of five helipot boxes there is a "five unit support." These various devices provide the necessary power and also contain provisions for checking and adjusting the performance. A responsivity equalizing system makes it possible 
to have all devices indicate on an identical $\mathrm{mr} / \mathrm{hr}$ basis, so that all can be connected to identical meters or recorders. A rack of 20 meters is provided; each has an adjustable trip-point, and when any meter is tripped a red pilot light just below: the meter lights up and a special alarm panel at the main control console lights up.

An integrating and printing device, made specially by Di-An Controls, Inc., samples and integrates the readings from each chamber, prints the cumulative total for each chamber every hour, and starts a new cycle every 24 hours. It does this for as many as 19 chambers.

Strip-chart recorders record the output of several of the chambers continuously, to show the dosage rates from minute to minute.

By 3/1/64 most of the system had been in use for 1 to 2 years, and, after some initial corrections, had performed well. A typical chamber is readily capable of detecting a change of a few percent in the cosmic ray background. Because the methane gas filling, and likewise the epoxy resin liner, contains much hydrogen, the chamber responds well (in terms of rads) not only to gamma radiation but also to neutron radiation; that is, it is approximately tissue-equivalent. In terms of rems, however, the response to neutrons is relatively low. Accordingly the chambers are used primarily as monitors of gamma radiation.

\subsection{Semi-Portable Area-Monitors}

On 3/1/64 ten Nuclear Measurements Corporation semi-portable area-monitors were on hand. Employing a sodium iodide crystal scintillator and a photomultiplier, each of these "modified Type GA-2A" devices indicates, on a 3-decade, logarithmic, 9-inch meter, any gamma radiation dose rate between 1 and 1000 millirads per hour. Atop the housing are three lamps (green, amber, red) that are energized when the radiation level exceeds the adjustable trip-point settings on a small internal meter; normally the settings are $5 \mathrm{mr} / \mathrm{hr}$ and $100 \mathrm{mr} / \mathrm{hr}$. The device reads average current from the photomultiplier, hence reads correctly even when the radiation comes in short bursts. The devices are mounted on stands and can be carried or wheeled to any desired location. They run off the $115 \mathrm{v}$, 60 cycle a.c. line.

\subsection{Biological Dose Meter Responsive to 0 to $15 \mathrm{Mev}$ Neutrons}

On 3/1/64 the CEA had on hand two Texas Nuclear Corporation Type 9120 meters for measuring the biological dose, in rems, due to 0 to $15 \mathrm{Mev}$ neutrons. The detector 
proper, a small crystal of europeum-doped $\mathrm{Li}^{6} \mathrm{I}$, is enclosed in a 12-inch-diameter sphere of polyethylene, which serves to slow the incident neutrons so that they may be detected by the $\mathrm{Li}^{6}(\mathrm{n}, a) \mathrm{H}^{3}$ reaction. The radius of the sphere is such that the probability that a given neutron incident on the sphere will produce a count is roughly proportional to the biological dose (in rems) that such a neutron delivers to a person whom it strikes. One rem corresponds to about 3000 counts. The device includes a discriminator. The " $Q$ " of the reaction is so large (greater than $4 \mathrm{Mev}$ ) that a large biasing voltage may be used a bias so great as to eliminate practically all response to gamma radiation.

On $3 / 1 / 64$ one of these "8-ball" devices was situated just below the ceiling of the Experimental Hall, near Beampath 8; the counter was situated in the Control Room. The other 8-ball device was situated just outside the Experimental Hall, atop the earth bank, near Beampath 8.

A somewhat similar device, called a Hankins-type monitor, was built at CEA and put into use in February 1963. It has a smaller (10"-diameter) sphere of Plexiglass, and is useful only in the 0 to $7 \mathrm{Mev}$ range. It has the advantage of being light-weight and battery operated, hence highly portable. It is useful in making surveys at outdoor locations.

\subsection{Ekco-Type Portable Survey Meters}

On 3/1/64 eleven portable Ekco Electronics, Ltd., N-555 beta gamma survey meters were on hand. These are used routinely in measuring radiation levels in the circular tunnel, Target Area, Experimental Hall, etc:

\subsection{Film Badges for Persons}

It has been the practice to issue film badges routinely to all persons who work in CEA areas that may contain radiation in amount exceeding $2 \frac{1}{2} \mathrm{millirad} /$ hour. Each badge contains a two-week film and a three-month film, which are developed and evaluated by the Harvard Radiological Services. .

\subsection{Film Packets Used as Area Monitors}

It has been the practice to use large quantities of film packets (about 50 packets, typically) as monitors for indicating the cumulative doses of gamma radiation at various sites outside the CEA buildings. Some are mounted on fences or trees near the various parking lots, and some are mounted in the nearby Biological Laboratory. 
21.8 Other RADSAF Equipment

On $3 / 1 / 64$ the following additional RADSAF equipments were on hand:

Two Nuclear-Chicago Corp. Model 2612 three-range beta-gamma survey meters employing GM counters.

One Nuclear-Chicago Corp. Model 2715 NEMO neutron survey meter.

One Victoreen-Jordan "Radgun" Model AGB-10KG-SR gamma radiation monitor. The device has a range of 9 decades.

Also a Tracerlab D-2X radiacmeter, a REAC Nu-Tec pocket monitor, about 30 Bendix \#862 pocket dosimeters (self-reading), about 200 Victoreen \#362-3A pocket chambers, a Victoreen 287 Minometer, two Bendix \#906/1 CD V-756 chargers, and various small radioactive sources for use in calibrating monitors.

\subsection{Success of Program}

The readings of the various area monitors have indicated that, in all work areas inside or outside the CEA buildings, no person could have received, in any quarter year, from radiation originating within the $\mathrm{CEA}$, a dose amounting to more than a small fraction of the permissible dose. The film badges indicated that no person received more than about one tenth of the permissible dose.

\section{$\underline{\text { Bibliography }}$}

H. R. Myers "Plans for radiation safety at the CEA," CEA-TM-69, June 1961.

W. A. Shurcliff "Status of radiation safety equipment in the hot half of the Experimental Hall," 11/27/62.

W. A. Shurcliff: CEA-RS Reports 1, 2, 3, 4, and also CEAL-TM Reports 101, 103, 105, 112 of 1962 and 1963.

G. Voss and H. Winick "Operations procedures relating to radiation safety," 12/20/63. 
$\underline{\text { Interlock System }}$

22.1 Main interlock system

22.2 Auxiliary interlock systems

22.3 Special access while linac is running

\subsection{Main Interlock System}

The purpose of the main interlock system is to help keep persons out of the synchrotron arca proper while the synchrotron is running, and yet permit convenient "special access" by authorized persons when the synchrotron is turned off temporarily. The system includes doors, switches, locks, keys, visual and aural warning devices, relays, indicators, and controls. The system was designed and built by the CEA staff, and has been modified from time to time to meet changing requirements. On $3 / 1 / 64$ it included the components discussed below.

Doors: Boundary doors or gates are provided at the south end of the labyrinth, just inside the west exit house (by the Linac Control Room), just inside the east exit house, and at the inner ends of the four radial tunnels. Partitioning gates are provided near Straight Sections 5 and 39 in the circular tunnel. Boundary doors keep people out of the synchrotron area; partitioning gates facilitate the tunnel search that must be made before the synchrotron can first be started, and in addition make it possible to exclude people from the linac area alone.

Limit switches: Limit switches are installed at each boundary door, to sense whether the door is open. The linac cannot run except when all doors are closed. If a door is opened at an inappropriate time, the switch operates a relay that shuts off the linac instantly, and the linac cannot be restarted until another tunnel search has been made.

Door latches: All boundary doors except one are provided with latches and locks to hold them shut. An exception is the door at the north corner of the Experimental Hall; here quick exit from the Experimental Hall into the interlocked area and east exit house area might be necessary if there were a sudden danger of a hydrogen explosion in the Experimental Hall; this door has a latch but no lock. In every case, a person inside the synchrotron area can open the door instantly, without use of a key. A person outside a locked door cannot open the door without using a chained key kept nearby. 
Chained key: Adjacent to each locked door there is a key-switch; the key is secured permanently by a long chain. The linac cannot operate unless every chained key is in its key-switch and is at "red" azimuth. One cannot withdraw a key (to unlock the door) unless one first turns the key to green. Turning it to green at an inappropriate time turns off the linac instantly. All chained keys are identical. Only the Electrical Engineering Division has spare keys.

Portable keys: Just inside the west exit house gate there are three keys that are situated in three wall switches and are normally at red azimuth. These keys are portable: they can be turned to green, removed, and pocketed. The linac cannot run unless every wall switch contains a key and every key is at red azimuth. When a man is to enter the linac area on special access, he may withdraw one of these keys and pocket it, thus insuring that nobody can start the linac; when he leaves the area, he restores the key. Wall. switches and portable keys are provided at every door where persons may be admitted on special access. All portable keys are identical. Only the Electrical Engineering Division has spare keys.

Flashing red beacons: Flashing red beacons are mounted at various locations within the synchrotron area. When the synchrotron is running or is about to run, these beacons flash. They are always off at those times when persons are permitted to be within the area in question.

Red panels: At each boundary door there is a large, internally illuminated red panel reading "Do not open door." The panel at any one door is dark at all times when persons are permitted to open this door but are illuminated when opening the door would require a new tunnel scarch.

Green panels: At each boundary door there is a large, internally illuminated green panel reading "Enter." It is dark when the red panel is illuminated, but is illuminated at . other times.

Red bulbs by doors: At the boundary doors there are red bulbs. Whenever the flashing red beacons are on, these red bulbs are lit to convey the information that the synchrotron is running or is about to run very soon and the synchrotron area is currently to be regarded as a very-high-level-radiation area, i.e., a no-access radiation area.

Crash buttons: Crash buttons are located at many places within the circular tunnel and Target Area. Pressing such a button shuts off the linac, the magnet power supply, and the rf power supply; the linac cannot be started again until a new tunnel search is made. 
Loud 'warning sound: A loud and disagreeable warning sound - a scream - may be heard throughout the synchrotron area during the one-minute period-of-grace that precedes start-up of the linac.

Confirmation buttons: Adjacent to each door there is a button that the tunnel searchers press in order to indicate that the door has been closed and the various keys are in place and are at red azimuth.

Tunnel search: Before the linac is turned on, two or three Operations Division men search the tunnels, etc., of the synchrotron area. They follow a prescribed route, secure all doors, and expel all people. The men start from one closed and locked partitioning gate; advancing methodically; searching carefully, expelling all people they meet, they close the various doors successively, press the respective confirmation buttons (and thereby illuminate the respective red panels successively), and eventually emerge at a point near the gate where the search began. Their task is facilitated by a check list indicating. the less-obvious tasks to be performed. The one-minute period-of-grace cannot start until the search has been completed successfully and all confirmation buttons have been pressed in the prescribed sequence.

Gentle warning sound: A gentle warning sound - beeping - may be heard throughout the synchrotron area when a tunnel search is in progress or a special access event is in progress.

One-minute period-of-grace: When the tunnel search has been completed successfully; a one-minute period-of-grace starts automatically; this provides a final emphatic warning to any persons still in the area and signifies that they should immediately leave the area or press a crash button to prevent start-up of the linac. During this one-minute period the red beacons flash, the loud and disagreeable warning occurs; and a majority of the white ceiling lights in the circular tuninel and target area are out. At the end of the period all the ceiling lights come on again, the acoustical warning ceases, and the beacons continue to flash.

Control room equipment: The Control Room includes an array of red pilot lights that show the conditions of the doors: if a given door is open (and/or if the portable keyswitch there is at "green" azimuth) the pertinent pilot light is on. Also there are lights showing whether a tunnel search is in progress, whether the linac is permitted to be running. In addition there are buttons for "enabling" the tunnel search to begin, enabling the linac, invoking the special access condition, and for permitting special access via a special access door. There are several such doors, each controlled. by a separate button. 
Special access: On certain occasions, specially authorized persons are allowed to enter the synchrotron area even after a tunnel search has been completed and even after the synchrotron has been running - without creating the need for a new tunnel search. Such special access may be requested by Operations Division men who wish to adjust the linac, the magnetic inflector, or other component, or by experimentalists who wish to adjust collimators, scrapers, detectors, etc. It is estimated that more than 1000 special access events took place in the first 18 months of synchrotron operation.

The procedure used in enabling special access to the synchrotron area is as follows. An Operations Division escort is assigned by the Operator to the group of men wishing to enter the area, and the escort tells the Operator (by walkie-talkie radio, usually) the names of the men concerned and states which special-access door is to be used. The Operator turns off the linac, records the names of the men, presses a "special-access condition" button, then presses a button that (a) makes it possible to open the door in question without breaking the interlock chain and (b) turns off the red panel adjacent to the door and turns on the green panel. The escort removes the chained key from the key-switch and unlocks the door. The men enter; the escort restores the key, closes the door, and informs the Operator that all men are inside. The Operator then releases the last-mentioned button. The escort removes and pockets one of the portable keys, and one or two other men may do likewise. The men then proceed to the appropriate area in the circular tunnel or Target Area and perform the intended task. On finishing this they leave the synchrotron area: they employ a procedure essentially the reverse of that followed in entering.

Logic diagrams: The relationships between key-switches, limit switches, specialaccess buttons, crash buttons, visual and aural warnings, etc., are indicated in detail in logic diagrams B-18E-19D and B-18E-22D, and in the diagrams included in CEAL-TM-114. See also CEAL-TM-130.

\subsection{Auxiliary Interlock Systems}

From time to time various Experimental Hall areas that are to contain high-energy beams and high-level radiation are demarcated and walled off by concrete blocks. This has been done, for example, for the Beam 7 area, the Beam 10 area, and other areas. At least one gate is provided to each such area.

Each such area is served by an auxiliary interlock system. A limit switch, special keys, a special light, and a warning sign are installed near the gate. A remotely controlled, 
in-vacuum shutter (described in Chap. 30) is installed in the pertinent beam path at a location just upstream from the Main Shielding Wall; the shutter is equipped with positionsensing switches tied in with the auxiliary interlock circuit. This circuit itself is tied in with the main interlock system in such manner that the opening of the gate or the shutter at an inappropriate moment turns off the linac instantly.

A special search procedure for the area in question is arranged, and also a specialaccess procedure patterned on that discussed in the previous section.

\subsection{Special Access While Linac Is Running}

Turning off the linac to permit men to enter the Target Area on special access entails loss of time, especially on occasions when the linac is not running smoothly. Accordingly, plans were made (in 1963) for providing an "extra-special access" procedure that makes it feasible to leave the linac running during a period of special access to the Target Area. Before the special-access group is allowed to enter this area, the beam emerging from the linac proper is diverted ("derailed") by the analyzing magnet to the Faraday cup; thus almost no radiation proceeds downstream as far as the Target Area. At the same time, a beam blocker (described in Chap. 9) is inserted in the beam transport pipe running from linac to synchrotron proper to provide added assurance that no beam can enter the synchrotron. Also, a radiation monitor situated well downstream from the linac is brought into play.

The protective circuit is such that the Operator cannot admit men to the Target Area - while the linac is running - unless three conditions are satisfied simultaneously: (1) a monitor of analyzing-magnet current indicates that the linac beam is deflected toward the Faraday cup, (2) a position sensor confirms that the beam blocker lies squarely in the path from linac to synchrotron, and (3) the radiation monitor indicates that, downstream from the linac, there is practically no radiation. If, after the men have been admitted to the Target Area, any of these three conditions ceases, the linac is turned off instantly, automatically.

On $3 / 1 / 64$ there was no urgent need for using the extra-special-access procedure, and there was no expectation of using it in the near future. 
Bibliography

H. R. Myers "Plans for radiation safety at the CEA," CEA-TM-69, June 1961.

G. R. Gonzalez, H. R. Myers, W. A. Shurcliff "Interlock system for synchrotron area," CEA-TM-78 of $3 / 15 / 62$ 。

G. R. Gonzalez "Interlock system for radiation protection," CEAL-TM-114 of 10/28/63. 


\section{Chapter 23}

\section{Miscellaneous Components of the Accelerator Proper}

23.1 Triggering systems

23.2 Closed circuit TV system

23.3 Audio intercommunication system

23.4 VHF radio communication system

23.5 Sound-powered interphone system

23.6 Spill indicator

23.7 Indicator of energy of orbiting electrons

23.8 Sequential beam-sharing system

23.9 Beam dumping system

23.10 Beam intensity limiter

23.11 Monitors of the external electron beam

23.12 System for exciting betatron oscillations

23.13 The 10-ft-modulus grid

23.14 Height of floor at locations of the brass plugs

23.15 Counting Room

23.16 CEA file of drawings

23.17 Safety equipment

\subsection{Triggering System}

Timing signals, or triggering signals, are required for many purposes at CEA. The most notable purpose is triggering the linac. The energy of the electrons that emerge from the linac is reasonably constant, but the field strength of the synchrotron magnets is constantly changing (in biased sinusoidal form, as explained in Chap: 6). If the electrons are to be injected into the synchrotron proper at the exact instant when electron energy and magnet excitation match, the timing of injection must be controlled accurately. Accuracy of about 1 or $2 \mu \mathrm{s}$ is required when the magnets are powered for 6-Bev operation, and an accuracy of 5 or $10 \mu \mathrm{s}$ is satisfactory during $1-\mathrm{Bev}$ operation. Timing signals are required also for initiating the modulation of the rf system, initiating the horizontal sweeps of various oscilloscopes, and controlling gating circuits associated with experimentalists' detectors.

A timing signal is usually derived from a "peaking strip" mounted between the jaws of one of the synchrotron magnets. A peaking strip is a small piece of high-permeability . metal that has (1) a nearly-square B-vs.-H hysteresis loop; i.e., a loop that has nearly vertical sides, and (2) a low coercive force. It may consist, for example, of a $1 / 2^{\text {n-long }}$ piece of 1-mil-diameter wire of specially treated molybdenum permalloy. When such a strip is placed between the jaws of an a.c.-excited magnet, a sudden reversal occurs in. 
the magnetization of the strip each time the field strength passes a certain value - a value that is approximately zero. The sudden reversal causes an electromagnetic pulse to be radiated, and this may be detected by a small enclosing pick-up coil. The current pulse from the coil is amplified, shaped to form a very short pulse, and used as a triggering signal. Usually the peaking strip proper is enclosed in a small coil carrying a (direct) biasing current; by adjusting this current the operator can cause the strip to "fire" at any desired magnet excitation up to, say, 40 gauss.

The linac triggering signal is derived from one or more peaking strips situated in one of the 48 magnets (Magnet 44 , on $3 / 1 / 64$ ); the strip is mounted close beside the pertinent vacuum chamber, i.e., about $3^{n}$ from the isomagnetic line. The signal from the pickup coil is approximately proportional to $\mathrm{dB} / \mathrm{dt}$ and amounts typically to a fcw millivolts. It is amplified, shaped, and transmitted to the Control Room via coaxial cable. A timing signal is then sent from there to the Linac Control. Room.

When tuning up the accelerator as a whole, the operator adjusts the peaking-strip biasing current by trial and error until the number of electrons captured into stable orbit is maximized.

Inevitably some delay occurs between the instant when the triggering signal is dispatched to the linac and the instant when electrons from the linac reach the synchrotron proper. In recent months (i.e., prior to March 1964) this delay amounted to 10 to $15 \mu \mathrm{s}$. Such a delay would be entirely acceptable if the excitation waveform of the magnets was the same in every cycle, since the operator could make due allowance for this once and for all. Actually, for reasons not fully understood, the excitation waveform changes significantly even within seconds.

Instead of readjusting the peaking-strip bias from moment to moment, the operatur can make use of a "double peaking-strip system," or. predicter system, designed and built in 1962. This employs two peaking strips, which are biased to different extents and hence fire at different times; the difference in time serves as an inverse measure of $\mathrm{dB} / \mathrm{dt}$ and forms the basis for automatic prediction, by a special electronic circuit, of the ideal moment at which to start the train of events culminating in the triggering of the linac. If the predicter is in proper adjustment, the electrons from the linac reach the synchrotron at approximately the ideal moment irrespective of any small changes in waveform of magnet excitation.

It would be ideal if the delay in question could be reduced to $5 \mu \mathrm{s}$, or even to $2 \mu \mathrm{s}$. There would then be no need for a double peaking-strip system; a single peaking strip 
would suffice and would seldom need to be readjusted. Since the recent introduction of a fast-acting 7-kw pulser (klystron) in the linac powering circuit, the path has been cleared for making a big reduction in the delay. It is expected that such a reduction will be accomplished soon.

On $3 / 1 / 64$ there were three peaking strips of standard design in the ring. All three were situated in Magnet 44.

\section{References:}

H. Nysater "Dynamic measurements with biased peaking strips of magnetic field and field gradient at low field," CEA-51 of $3 / 28 / 58$.

\subsection{Closed Circuit TV System}

The CEA closed circuit TV system, used in monitoring targets, entranceways to dangerous areas, and active experimental areas, consists of six TV cameras and six display units, or monitors. Cables connect the Control Room with sixteen different stations in the Circular Tunnel, Target Area, and Experimental Hall; TV cameras can be installed quickly in the neighborhood of any of these stations with the aid of 100-ft extension cables.

Operators in the Control Room make routine use of several of the TV cameras, and can control the pan angle, tilt angle, focal length (zoom), optical focus, electrical focus, iris diameter, target potential, beam brightness, and black-level of each such camerá. Cameras not used by Control Room personnel are available for use by experimentalists; several monitors and control units are available in the Counting Room.

\section{References:}

L. A. Law "CEA closed circuit television system," CEA-TM-87 of 6/27/62.

L. A. Law "Machine signals available to experimental groups, closed circuit television and communication, beam bumps and machine sharing," informal report of 9/4/63.

\subsection{Audio Intercommunication System}

The audio intercommunication system is a telephone system that is used in conjunction with the closed circuit television system. It was purchased from the Centrum Corp., and includes ten "Futura" send-and-receive boxes and a central amplifier. The equipment may be used in station-to-station mode or in conference mode.

\section{References:}

L. A. Law "Centrum audio communication system," CEA-TM-82 of 5/21/62. 


\subsection{VHF Radio Communication System}

The VHF radio communication system is used in maintaining communication between the Control Room and locations where technicians are making tunnel searches, adjusting targets, etc.

The equipment was purchased from Motorola, Inc. The system includes five base stations, and considerably larger numbers of pocket transmitters and pocket receivers. Two of the base stations are in routine use; they are situated in the Control Room and the Counting Room.

The (crystal-controlled) transmitter frequencies lie between 150 and $160 \mathrm{Mc}$, in the Experimental Research Band, and are used under FCC license.

References:

J. Fitzgerald "VHF facility, Room 104," CEAL-TM-106 of 6/21/63.

\subsection{Sound-Powered Interphone System}

The sound-powered interphone system is used in maintaining communication between a large number of work areas and the various control rooms. It includes 63 jackbox stations. There is one station at each of the 48 magnets in the ring; other stations are situated in the Target Area and the various control rooms. The system includes a general circuit common to all stations and 16 private circuits connecting certain specially designated stations. The master terminal box is situated in the Control Room.

Refereities.

R. Fucaloro "CEA sound-powered interphone system," CEA-TM-81 of 3/15/62.

\subsection{Spill Indicator}

Most users of a CEA external beam of high-energy photons or electrons want the time interval in which the external beam exists (in each cycle of synchrotron operation) to be at least as great as $1 / 2 \mathrm{~ms}$ and perhaps even as great as 1 or $2 \mathrm{~ms}$. If the spill time is long, the users' detectors are less likely to be overloaded and the number of spurious coincidence is small:

A spill indicator is used to show the actual length of the spill time and to give some idea as to the uniformity of intensity within this interval. The heart of the indicator 
is a scintillator, which is placed near a target or collimator or other object struck by the beam. Radiation scattered by such object produces light in the scintillator, the light is detected by a photomultiplier, and the output signal is fed to a CRO located in the Control Room or elsewhere.

On $3 / 1 / 64$ there were about 12 spill detectors mounted adjacent to the synchrotron ring. Each served a different straight section at which an external beam could originate.

\subsection{Indicator of Energy of Orbiting Electrons}

In the summer of 1963 a device for computing the effective strength of the magnetic field in the ring of 48 magnets was built. Called a "B-dot integrator," the device not only (1) computes the ficld strength that exists at any instant, but also (2) produces a signal each time the field strength increases by a specified increment such as 6 gauss, and (3) actuates a meter calibrated directly in energy of the orbiting electron, in Bev.

The dcvice empluys a B-dot sensor consisting of a small, multi-turn coil of wire mounted within the gap of one of the 48 magnets; it is attached to the top of a vacuum chamber, and hence is situated about $3 / 4$ inch above the isomagnetic line. The resulting instantaneous voltage is fed. to a voltage-to-frequency converter the output of which is fed to a counter. A gate circuit triggered by a peaking strip causes the counting to start at the instant the field has the value zero and is increasing. The overall responsivity is adjusted so that a peak field appropriate to an electron energy of $6 \mathrm{Bev}$ produces 6000 counts.

\subsection{Sequential Beam-Sharing System}

When two (or possibly more) groups of experimentalists wish to operate concurrently, the synchrotron operator makes use of a sequential beam-sharing system, or logic circuit, that causes the orbiting beams in successive pulses of the accelerator to strike targets in different straight sections, according to the respective needs and priorities of the two groups of users. The system is applicable if the beam-bump method of target engagement is used by one, or both, of the users. The logic circuit was designed and built by CEA engineers in 1963 and 1964 and is still being extended and improved. Only a brief description is presented here. Further details may be found in the memoranda listed at the end of this section.

The sequential beam-sharing system can operate in any of several modes. When it is operated in fast alternating mode, User $A$ is assigned pulses according to any of a great 
variety of schedules, and User $B$ is assigned the rest of the pulses. The various schedules apply to a group of 64 pulses, rather than 60, since flip-flops are employed and six of these can provide 64 combinations; i.e., $2^{6}=64$. In one schedule, pulses $1,3,5, \ldots$ through 63 may be assigned to User A. In another schedule pulses 1, 5, 9, etc. may be assigned to him. Other schedules involve pulses 1, 9, 17, etc., or pulses 1, 17, 33, etc., or pulses 1 and 33, or, finally, just pulse 1. In other words User A may be given 32, $16,8,4,2$, or 1 pulse out of every group of 64 , and User $B$ gets the rest.

When the beam-sharing system is operated in single-pulse demand mode, User A receives, on demand communicated by a special triggering signal, any single pulse that he wishes, and the remaining pulses go to User $B$. There is no limitation on the number of pulses or sequence of pulses that User A can demand; but he must dispatch a separate triggering signal for each pulse wanted. This mode is employed by the Bubble Chamber Group; whenever the bubble chamber is ready for a pulse, a demand signal is dispatched and a pulse is received. Usually about 1 or 2 pulses are demanded per second.

When the steady demand mode is used, User A receives an uninterrupted succession of pulses as long as a steady demand signal is maintained, and User B receives the pulses that occur when the demand signal is absent. By $3 / 1 / 64$ this mode had been made nearly ready for use, but had not actually been put to use.

The sequential beam-sharing system governs only the allocation of the pulses to the different straight sections concerned. Other equipment makes it possible for each user to obtain the desired amplitude and phase of beam-bump, irrespective of the amplitude and phase desired by the other user.

\section{References:}

L. A. Law "Machine signals available to experimental groups, closed circuit television and communication, beam bumps and machine sharing," informal memorandum of 9/4/63.

R. I. Samuel "Beam bump computer," informal memorandum of 12/30/63.

\subsection{Beam Dumping System}

Provision has been made whereby the pulses allocated to a given straight section (and given experimentalist) may be disposed of, or dumped, at a location far removed from the Target Area and without the need for changing the operation of the linac or other major component of the synchrotron. Dumping may be invoked whenever the pertinent experimental area in the Experimental Hall is to be made safe for temporary entry by the experimentalist 
concerned; the accelerator operator dumps the pertinent pulses, closes the pertinent shutter in the Main Shielding Wall, then allows the experimentalist to enter.

Dumping is accomplished by energizing back-leg windings that produce a beam-bump at Straight Section 19 and cause the orbiting electrons to strike a scraper there. Dumping occurs soon after the start of the acceleration interval, i.e., while the electron energy is still relatively low. The set of back-leg windings used is similar to the sets described in Chap. 16.

\subsection{Beam Intensity Limiter}

Design work is underway on a system that will limit the intensity of the orbiting beam of electrons to a specified value. If a given experimentalist has adjusted his equipment so that it performs in optimum manner when the intensity is, say, 2 ma, he may find it harmful for occasional pulses to have intensities as great as 3 or 4 ma. The proposed beam-intenstty limiter will reduce the intensity of such occasional pulses to the prearranged limit. To accomplish the reduction, the operator will employ a beam bump to make the beam graze a scraper to just such an extent that the intensity is reduced to the desired value. This will be done during the first half of the acceleration interval. The scraper will perhaps be located in Straight Section 19.

\subsection{Monitors of the External Electron Beam}

On $3 / 1 / 64$ various kinds of monitors of the external electron beam were being readied for use. One type, mounted in a tank similar to a regular straight section tank, employs coils much like those used in the small beam-intensity monitor discussed in Chap. 14; however, the circuit is tuned to the orbit frequency $(1.32 \mathrm{Mc} / \mathrm{sec})$ in order that the necessary increase in responsivity can be achieved. Another type consists essentially of a resonant cavity (spare rf cavity) such as is discussed in Chap. 14. Another type employs a small ferrite toroid. Experimentalists who wish to determine beam intensity with high accuracy will presumably use Faraday cups or secondary-emission monitors such as are described in Chap. 30.

Transverse displacements of the external electron beam can be measured with the aid of a small coil system that is tuned to the orbit frequency.

\subsection{System for Exciting Betatron Oscillations}

When the accelerator was first started up, the designers wished to know whether the frequencies of vertical and horizontal betatron oscillations had the intended value, 
namely about 6.4 per turn. With respect to electrons having high (3 to $6 \mathrm{Bev}$ ) energy, a single evaluation sufficed, since there was no likelihood that the value would change. With respect to electrons having very low energy, i.e., 20 to $100 \mathrm{Mev}$, it was necessary to evaluate the frequencies under a variety of conditions, including (1) different injection energies, (2) different values of $\mathrm{dB} / \mathrm{dt}$ at injection, (3) different times in the early portion of the acceleration interval, (4) different extents of excitation of the various kinds of pole face windings.

The betatron oscillation frequencies are evaluated conveniently with the aid of equipment that excites, or augments, the oscillations coherently-and equipment that detects the augmentation. Vertical betatron oscillations are excited with the aid of (a) a coil system mounted in Straight Section Tank 40, (b) an accurately calibrated, variable-frequency oscillator operating in the range from 0.2 to $1.0 \mathrm{Mc} / \mathrm{sec}$, (c) a bruad-band power amplifier, and (d) a variable width gate and variable delay, unit. When the coils are excited they urge the passing electrons up or down. Their effect is coherent, cumulative, and very pronounced if the frequency of the oscillator coincides with one of a set of special frequencies. $\left(\nu_{z}-n\right) f_{0}$ where $\nu_{z}$ is the frequency of vertical betatron oscillation, $n$ is a positive or negative integer, and $f_{0}$ is the orbital frequency (about $1.32 \mathrm{Mc} / \mathrm{sec}$ ).

If $\mathrm{n}$ is chosen to be 6 and $\nu_{\mathrm{z}}$ happens to be exactly 6.4 , the special value of oscillator frequency is $(0.4) \mathrm{f}_{\mathrm{o}}$ or about $0.53 \mathrm{Mc} / \mathrm{sec}$. If the coil system is excited at this frequency the resulting coherent, vertical betatron oscillations of the electrons passing thruugh can be detected easily. If the energy of the electrons is low the oscillations become so large that most of the electrons are soon lost to the walls of the vacuum ring (despite the adiabatic damping discussed in Chap. 3). and the beam intensity drops almost to zero. The drop is easily observed with the aid of a CRO display of the signal from one of the beam-intensity monitors or vertical displacement monitors discussed in Chap. 14. If the electron energy is several Bev, the coherent oscillations are not large enough to reduce the intensity appreciably; however, the magnitude of the oscillations can be judged by observing a CRO display of the signal from a vertical displacement monitor.

The operator's procedure is to shift the oscillator frequency slowly and record that particular value $\mathrm{f}_{\max }$ that causes a maximum amplitude of coherent betatron oscillation. Then $\nu_{z}$ is found from the relation: $\nu_{z}=n+\mathrm{f}_{\max } / \mathrm{f}_{0}$.

The frequency of horizontal betatron oscillations is evaluated by a similar procedure. The excitation coils are mounted in Straight Section Tank 46.

The actual frequencies found are discussed in Chapters 7 and 27. 


\subsection{The 10-ft-Modulus Grid}

The 10-ft-modulus grid of brass plugs on the floor of the Experimental Hall and Target Area permits experimentalists to place their equipment in precise relationship to the synchrotron proper even when direct line-of-sight to the synchrotron is blocked by intervening equipment or by the Main Shielding Wall.

Constructed in 1961, the grid consists of approximately 500 brass plugs recessed in the concrete floor and punch-marked to indicate the exact spots intended - with an accuracy of about $1 / 8$ inch. Large painted numbers adjacent to each plug indicate the $X$ and $\mathrm{Y}$ coordinates. The $\mathrm{Y}$-axis is the straight horizontal line that (1) passes through the accelerator centerpoint and (2) lies in the vertical plane that contains the accelerator centerpoint and also that apex (of the 96-sided polygon) that lies in Straight Section Tank 10. The azimuthal location of this plane was determined with the aid of the four radial-survey fixture plates discussed in Chap. 5. The plates were mounted in standard manner on Magnets $8,9,10$, and 11 , and, with the aid of a tape, the surveyor marked on the floor a point equidistant from the inner stations of the fixture plates on Magnets 9 and 10. This point serves as origin of coordinates. The $\mathrm{X}$-axis is a straight horizontal line lying in the vertical plane that contains the line-of-sight joining the outer stations of the Magnet 8 and Magnet 11 fixture plates. It may be shown that the $\mathrm{X}$-axis is nominally 14.026 inches from the above-mentioned apex, 15.169 inches from the centerpoint of the above-mentioned tank, and 1435.870 inches from the accelerator centerpoint. The positive Y-axis runs approximately north; the positive $\mathrm{X}$-axis runs approximately east and passes almost through the extreme northeast corner of the Experimental Hall.

\subsection{Height of the Floor at Locations of the Brass Plugs}

In 1962 the height of the Experimental Hall floor and Target Area floor was measured at each plug location - more specifically, at a location 2 inches in the plus-Y direction from the punchmark on the plug. The height was compared with that of a nominal plane 60.00 inches below the mechanical median plane of the set of 48 magnets. The results are shown in Drawing E-21M-166A-WS; see also CEA-TM-88. Typically, the floor is $1 / 2$ to 1 inch lower than the nominal plane; the highest plug location coincides with that plane and the lowest plug location is 1.29 inches below it. In many areas the floor slopes considerably; the height changes by more than $1 / 2$ inch in ten feet. of travel along the floor. 
The height changes when the floor is loaded. In one test, a $20 \times 20 \times 10 \mathrm{ft}$., 500-ton pile of shielding blocks depressed the floor $1 / 8$ inch. In another test, a $36 \times 36 \times 16 \mathrm{ft}$., 2500-ton pile depressed it one inch. When a large load is removed, the floor height reverts part way toward the earlier value.

\subsection{Counting Room}

In 1963 the recording and analysis of data from the experimentalists' installations in the Target Area and Experimental Hall was centered in Room 104 of the Laboratory Building. It soon became evident, however, that more space was required. Accordingly a much larger Counting Room was constructed just above the Target Area - in the latter half of 1963. The room is equipped with windows by which the experimentalists can look down into the Experimental Hall to observe their equipment.

Much effort was given to including sufficient shielding in the floor of the new Counting Room (i.e., the ceiling of the Target Area). The goal was to provide enough shielding so that even if the full-intensity $6-\mathrm{Bev}$ beam of orbiting electrons struck a massive target just below the Counting Room, the dose rate in the Counting Room would be acceptable. An analysis of the requirements was made in March 1963; see an informal memorandum of $3 / 26 / 63$ by J. C. Street. As finally constructed, the floor of the Counting Room was 80 inches thick; it contained these layers of shielding:

2 inches of ordinary concrete (uppermost layer)

33 inches of ilmenite-loaded concrete

15 inches of iron billets

30 inches of ordinary concrete (forming the original ceiling of the Target Area)

Radiation surveys made in the new Counting Room show that, during normal operation of the synchrotron, the radiation in that room is too weak to be detected.

\subsection{CEA File of Drawings}

The CEA Mechanical Engineering Division maintains a file of drawings of accelerator components, buildings, and apparatus that is not a part of the accelerator. Each drawing bears a symbol which includes a preliminary letter indicating the size (A is very small, E very large), a preliminary pair of digits indicating the subject, and a serial number. The significance of the preliminary pair of digits is as follows:

11: Magnet and magnet coils

12: Magnet power supply, cooling, cooling tower

13: RF system including cavities and waveguides
15: Vacuum system and straight section tanks

16: Controls, pick-up coils, and other special equipment

21: Buildings and shielding

35: Surveying and miscellaneous

14: Linac and injection system 
The letters $\mathrm{M}$ or $\mathrm{E}$ appended to the preliminary pair of digits show whether the drawing pertains to a mechanical or electrical component.

No preliminary pair of digits is assigned to a drawing of a piece of apparatus that is not a part of the accelerator.

Revised drawings are indicated by letters appended to the serial number.

For further details see CEA-TM-21.

\subsection{Safety Equipment}

Much of the safety equipment at CEA pertains to radiation and is discussed in Chapters 21 and 22. Safety equipment pertinent to liquid hydrogen targets and bubble chambers is discussed in Chap. 29.

The CEA has no division concerned exclusively with safety, but operates under the general rules promulgated by the Division of Environmental Health and Safety of the Harvard University Health Services. Representatives of that Division, and also representatives of the corresponding group at M.I.T., make occasional inspections of CEA facilities and equipment and, where appropriate, make suggestions as to improved equipment and practices. The CEA staff attempts also to conform to general safety regulations established by the Atomic Energy Commission and periodic inspections are made by representatives of the Commission. 


\section{Chapter 24 \\ Photon Beams from Machine Targets}

24.1 Introduction

24.2 Predicted paths of the photon beams

24.3 Miscellaneous properties of the photon beams

\subsection{Introduction}

During the first two years of operation of the CEA, the great majority of experiments performed here employed high-energy photon beams (bremsstrahlung beams) that originated within the vacuum ring of the accelerator, emerged roughly tangentially from. the ring, passed through prearranged holes in the Main Shielding Wall, and entered the Experimental Hall. By 3/1/64 plans for ejecting the orbiting electrons themselves and conveying them into the Experimental Hall were well advanced, but no such beam had yet been made available in the Experimental Hall. One group had introduced its hydrogen target into one of the straight section tanks and studied the interaction of the primary electron beam with this target. The fact remained, however, that most of the beams used by experimentalists were photon beams originating at small tungsten targets situated within the vacuum ring.

\subsection{Predicted Paths of the Photon Beams}

Since the path of a given beam of bremsstrahlung photons lies along the extension to the line-of-flight of the electrons striking the target, the problem of calculating the axis of the photon beam reduces to that of calculating the tangent line to the path of a typical electron at the point where the electron strikes the target. If the target were situated on the centerline of the straight section concerned, the line in question would be essentially identical to this centerline. Usually the target is situated roughly 1 or $1 \frac{1}{2}$ inches to one side of the centerline; ordinarily it is situated on the inner side, i.e., the side toward the accelerator centerpoint. Accordingly (for reasons implied by the Chap. 3 discussions of betatron oscillations and beta function) the pertinent segment of the electron path is not parallel to the centerline but, rather, makes a slight angle with it. The magnitude of the angle depends on the cause of the electron's 1 or $1 \frac{1}{2}$ inch excursion.

One way of displacing the electron's path laterally by 1 or $1 \frac{1}{2}$ inches is to bump the pertinent portion of the equilibrium orbit in the manner described in Chap. 16. The shape 
and azimuth of the typical bump is such that a straight line that is tangent to the bump at the target location intersects the centerline of the straight section at a point (focal point) about $144^{\prime \prime}$ away, according to results presented by T. L. Collins in an informal memorandum of about $6 / 23 / 61$. The focal point is situated downstream or upstream from the target depending on whether the target is in a straight section immediately downstream from a closed magnet or an open magnet. The target may be on the inner side or the outer side of the usual equilibrium orbit.

Another way of displacing the electron's path laterally is to employ the rf-turn-off method of target engagement, also described in Chap. 16. As the electron loses energy and spirals inward, the shape of the equilibrium orbit changes and the tangent line at the target intersects the straight section centerline at a point 173" away, according to results presented in CEA-A-29. Again the focal point is downstream or upstream depending on whether the target is just downstream from a closed magnet or open magnet.

The above-mentioned focal-point distances of 144 and 173 inches must be regarded as approximations only, since they were derived without reference to horizontal betatron oscillations. Yet, when the accelerator is operated at 6-Bev level, such oscillations at the end of the acceleration interval are quite large, as indicated in Chap. 3. The first electrons to strike the target are those that have the largest oscillations. Experience indicates that, irrespective of what type of larget engagement method is used, the focal-point distances are specified with adequate accuracy by the single figure of 156 inches.

In an informal memorandum of 1961, T. L. Collins reported calculations as to the expected locations of the tangent lines pertinent to the two methods of target engagement and to the eight straight sections of principal interest to experimentalists. Later $(7 / 6 / 62)$ the results were made more widely available in an informal memorandum edited by W. A. Shurcliff.

Table 24.1 presents data as to the locations of each pertinent apex of the basic 96-sided polygon. Also it indicates the locations of the centerpoints of the pertinent straight sections. Locations are expressed in terms of the cartesian coordinates associated with the 10-ft-modulus grid described in Chap. 23. The table also shows the constants $\underline{a}$ and $\underline{b}$ whereby the centerline of a given straight section may be specified as: $Y=a+b X$. The centerline is, of course, perpendicular to the radius vector from accelerator centerpoint to the pertinent apex of the polygon.

Table 24.2 presents data as to target location $\left(\mathrm{X}_{\mathrm{t}}, \mathrm{Y}_{\mathrm{t}}\right)$ for a target that is $1.4^{\text {n }}$ radially inward or radially outward in the straight section concerned and gives values 
Table 24.1

Basic Data on Straight Sections

(Note: $\mathrm{X}, \mathrm{Y}$, and $\underline{\mathrm{a}}$ are in feet; $\underline{\mathrm{b}}$ is a pure number)

\begin{tabular}{|c|c|c|c|c|c|c|c|}
\hline \multicolumn{2}{|c|}{ Straight Section } & \multirow{2}{*}{\multicolumn{2}{|c|}{$\begin{array}{c}\text { Apex } \\
\text { coordinates }\end{array}$}} & \multirow{2}{*}{\multicolumn{2}{|c|}{$\begin{array}{l}\text { Centerpoint } \\
\text { coordinates }\end{array}$}} & \multirow{2}{*}{\multicolumn{2}{|c|}{$\begin{array}{c}\text { Centerline } \\
\text { coordinates }\end{array}$}} \\
\hline $\begin{array}{c}\text { Serial } \\
\text { Number }\end{array}$ & Azimuth & & & & & & \\
\hline 4 & $-45^{\circ}$ & $\begin{array}{l}X \\
Y\end{array}$ & $\begin{array}{r}-83.7830 \\
35.8730\end{array}$ & $\begin{array}{l}\mathrm{X} \\
\mathrm{Y}\end{array}$ & $\begin{array}{r}-83.716 \\
35.940\end{array}$ & $\begin{array}{l}\mathrm{a} \\
\mathrm{b}\end{array}$ & $\begin{array}{l}-47.7767 \\
-1.0000\end{array}$ \\
\hline 5 & $-37.5^{\circ}$ & $\begin{array}{l}X \\
Y\end{array}$ & $\begin{array}{r}-72.1303 \\
25.6539\end{array}$ & $\begin{array}{l}X \\
Y\end{array}$ & $\begin{array}{r}-72.072 \\
25.729\end{array}$ & $\begin{array}{l}\mathrm{a} \\
\mathrm{b}\end{array}$ & $\begin{array}{l}-29.573 \\
-\quad 0.76732\end{array}$ \\
\hline 7 & $-22.5^{\circ}$ & $\begin{array}{l}X \\
Y\end{array}$ & $\begin{array}{r}-45.3430 \\
10.1883\end{array}$ & $\begin{array}{l}X \\
Y\end{array}$ & $\begin{array}{r}-45.307 \\
10.276\end{array}$ & $\begin{array}{l}\mathrm{a} \\
\mathrm{b}\end{array}$ & $\begin{array}{l}-8.4900 \\
-0.41420\end{array}$ \\
\hline 8 & $-15^{\circ}$ & $\begin{array}{l}\mathrm{X} \\
\mathrm{Y}\end{array}$ & $\begin{array}{r}-30.6667 \\
5.2063\end{array}$ & $\begin{array}{l}\mathrm{X} \\
\mathrm{Y}\end{array}$ & $\begin{array}{r}-30.642 \\
5.298\end{array}$ & $\begin{array}{l}a \\
b\end{array}$ & $\begin{array}{l}-2.9121 \\
-0.26795\end{array}$ \\
\hline 10 & $0^{\circ}$ & $\begin{array}{l}X \\
Y\end{array}$ & $\begin{array}{l}0.0000 \\
1.169\end{array}$ & $\begin{array}{l}\mathrm{X} \\
\mathrm{Y}\end{array}$ & $\begin{array}{l}0.000 \\
1.264\end{array}$ & $\begin{array}{l}a \\
b\end{array}$ & $\begin{array}{l}1.2640 \\
0.0000\end{array}$ \\
\hline 11 & $7.5^{\circ}$ & $\begin{array}{l}\mathrm{X} \\
\mathrm{Y}\end{array}$ & $\begin{array}{r}15.4657 \\
2.1827\end{array}$ & $\begin{array}{l}\mathrm{X} \\
\mathrm{Y}\end{array}$ & $\begin{array}{r}15.453 \\
2.277\end{array}$ & $\begin{array}{l}\mathrm{a} \\
\mathrm{b}\end{array}$ & $\begin{array}{l}0.2425 \\
0.13165\end{array}$ \\
\hline 13 & $22.5^{\circ}$ & $\begin{array}{l}\mathrm{X} \\
\mathrm{Y}\end{array}$ & $\begin{array}{l}45.3430 \\
10.1883\end{array}$ & $\begin{array}{l}\mathbf{X} \\
\mathbf{Y}\end{array}$ & $\begin{array}{l}45.307 \\
10.276\end{array}$ & $\begin{array}{l}\mathrm{a} \\
\mathrm{b}\end{array}$ & $\begin{array}{ll}- & 8.4900 \\
& 0.41420\end{array}$ \\
\hline 14 & $30^{\circ}$ & $\begin{array}{l}\mathbf{X} \\
\mathbf{Y}\end{array}$ & $\begin{array}{l}59.2435 \\
17.0432\end{array}$ & $\begin{array}{l}\mathbf{X} \\
\mathbf{Y}\end{array}$ & $\begin{array}{l}59.196 \\
17.126\end{array}$ & $\begin{array}{l}a \\
b\end{array}$ & $\begin{array}{r}-17.0513 \\
0.57735\end{array}$ \\
\hline
\end{tabular}

$\underline{\mathrm{a}}$ and $\underline{\mathrm{b}}$ that permit one to specify the axis of the photon beam as: $\mathrm{Y}=\mathrm{a}+\mathrm{bX}$. Usually the target is 6" upstream or 6" downstream from the mid-cross-sectional plane of the straight section; but this fact has little significance and the figures presented in the tables are reasonably valid even if it is disregarded.

If the target position is closer to the orbit by, say, $0.4 "$, the location of the beam is easily found by "rotating" the beam specified in the table about the focal point.

Since the magnet ring is horizontal, the bremsstrahlung beams are approximately horizontal also. They may depart slightly from the horizontal if there is a vertical distortion of the orbit in the neighborhood of the target. In 1963, indication was obtained that Photon Beam 10 ran very slightly uphill, being about $\frac{1}{2}^{n} \pm \frac{1}{2}$ " above orbit height at a distance $100 \mathrm{ft}$. downstream from the target. 
Table 24.2

Coordinates $\mathrm{X}_{\mathrm{t}}$ and $\mathrm{Y}_{\mathrm{t}}$ of Target and Constants $\mathrm{a}$ and $\mathrm{b}$ $\therefore$ Pertinent to Axis of Photon Beam

(Note: The data refer to two methods of target engagement. The words in and out refer to displacements toward and away from the accelerator centerpoint. $X_{t}, \bar{Y}_{t}$, and $\bar{a}$ are in feet; $b$ is a pure number.)

\begin{tabular}{|c|c|c|c|c|}
\hline \multirow{2}{*}{$\begin{array}{l}\text { Straight } \\
\text { section } \\
\text { number }\end{array}$} & & \multicolumn{2}{|c|}{ Beam-Bump Engagement } & \multirow{2}{*}{$\begin{array}{l}\text { RF Turn-Of } \\
\text { when target } \\
\text { is } 1.4^{\prime \prime} \text { in }\end{array}$} \\
\hline & & $\begin{array}{c}\text { when target } \\
\text { is } 1.4^{n} \text { in }\end{array}$ & $\begin{array}{l}\text { when target } \\
\text { is } 1.4^{\prime \prime} \text { out }\end{array}$ & \\
\hline 4 & $\begin{array}{l}X_{t} \\
Y_{t} \\
a \\
b\end{array}$ & $\begin{array}{l}-83.633 \\
36.023 \\
-49.242 \\
-1.01963\end{array}$ & $\begin{array}{l}-83.799 \\
35.857 \\
-46.327 \\
-0.98074\end{array}$ & $\begin{array}{c}-83.633 \\
36.023 \\
-48.974 \\
-1.0163\end{array}$ \\
\hline 5 & $\begin{array}{l}\mathrm{X}_{\mathrm{t}} \\
\mathrm{Y}_{\mathrm{t}} \\
\mathrm{a} \\
\mathrm{b}\end{array}$ & $\begin{array}{l}-72.001 \\
25.822 \\
-28.323 \\
-0.75200\end{array}$ & $\begin{array}{c}-72.143 \\
25.636 \\
-30.843 \\
-0.78288\end{array}$ & $\begin{array}{l}-72.001 \\
25.822 \\
-28.506 \\
-0.75455\end{array}$ \\
\hline 7 & $\begin{array}{l}\mathrm{X}_{\mathrm{t}} \\
\mathrm{Y}_{\mathrm{t}} \\
\mathrm{a} \\
\mathrm{b}\end{array}$ & $\begin{array}{l}-45.262 \\
10.384 \\
-\quad 7.850 \\
-0.40286\end{array}$ & $\begin{array}{l}-45.352 \\
10.168 \\
-9.136 \\
-0.42564\end{array}$ & $\begin{array}{l}-45.262 \\
10.384 \\
-7.936 \\
-0.40476\end{array}$ \\
\hline 8 & $\begin{array}{l}X_{t} \\
Y_{t} \\
a \\
b\end{array}$ & $\begin{array}{l}-30.612 \\
5.411 \\
-3.111 \\
-0.27839\end{array}$ & $\begin{array}{l}-30.672 \\
5.185 \\
-2.715 \\
-0.25755\end{array}$ & $\begin{array}{l}-30.612 \\
\quad 5.411 \\
-3.058 \\
-0.27664\end{array}$ \\
\hline 10 & $\begin{array}{l}X_{t} \\
Y_{t} \\
a \\
b\end{array}$ & $\begin{aligned} & 0.000 \\
& 1.381 \\
& 1.381 \\
&- 0.00972\end{aligned}$ & $\begin{array}{l}0.000 \\
1.147 \\
1.147 \\
0.00972\end{array}$ & $\begin{aligned} & 0.000 \\
& 1.381 \\
& 1.381 \\
- & 0.00809\end{aligned}$ \\
\hline 11 & $\begin{array}{l}X_{t} \\
Y_{t} \\
a \\
b\end{array}$ & $\begin{array}{l}15.438 \\
2.393 \\
0.207 \\
0.14155\end{array}$ & $\begin{array}{l}15.468 \\
2.161 \\
0.277 \\
0.12177\end{array}$ & $\begin{array}{l}15.438 \\
2.393 \\
0.234 \\
0.13988\end{array}$ \\
\hline 13 & $\begin{array}{l}X_{t} \\
Y_{t} \\
a \\
b\end{array}$ & $\begin{array}{c}45.262 \\
10.384 \\
-\quad 8.881 \\
0.42564\end{array}$ & $\begin{array}{c}45.352 \\
10.168 \\
-\quad 8.103 \\
0.40286\end{array}$ & $\begin{array}{c}45.262 \\
10.384 \\
-8.794 \\
0.42371\end{array}$ \\
\hline 14 & $\begin{array}{l}\mathrm{X}_{\mathrm{t}} \\
\mathrm{Y}_{\mathrm{t}} \\
\mathrm{a} \\
\mathrm{b}\end{array}$ & $\begin{array}{c}59.138 \\
17.227 \\
-16.154 \\
0.56446\end{array}$ & $\begin{array}{c}59.255 \\
17.025 \\
-17.958 \\
0.59038\end{array}$ & $\begin{array}{c}59.138 \\
17.227 \\
-16.281 \\
0.56661\end{array}$ \\
\hline
\end{tabular}




\subsection{Miscellaneous Properties of the Photon Beams}

Because electrons that strike a tungsten target are multiply scattered within it, the resulting bremsstrahlung beam has appreciable angular width. The width varies inversely with the energy of the incident electrons. If the target thickness is $0.1 \mathrm{X}_{\mathrm{o}}$ (see Chap. 16) and the electron energy is $6 \mathrm{Bev}$, the "full width at half-height" of the photon beam is of the order of 2 milliradians. The width is greater if a thick target is used, or if the electrons strike the target in a variety of directions, or if the photon beam emerges from the vacuum ring via a thick window.

If the target thickness is $0.1 \mathrm{x}_{\mathrm{o}}$, the number of equivalent quanta in the photon beam is about one tenth of the number of electrons striking the target. Thus if the beam intensity has the design value of $10^{11}$ electrons per pulse, about $10^{10}$ equivalent quanta are produced per pulse. (Number of equivalent quanta means the ratio of energy carried by the bremsstrahlung beam to the energy of the individual electron that strikes the target. It may be regarded as the number of photons that would be produced if every photon had the maximum energy.) Actually, the number of photons in the beam is much greater than the number of equivalent quanta, and all photon-energies, from zero to the electron energy, occur in roughly equal abundance.

The 100-to-200 ft. gap in the orbiting electron beam of electrons leads to a corresponding gap in the photon beam. Also, the $\mathrm{rf}$ structure of the orbiting beam, that is, the bunching with a spacing of the order of $2 \mathrm{ft}$., leads to a similar structure of the photon beam if the beam-bump method of target engagement is used, i.e., if the rf power level remains high and the electrons remain captive within $r f$ buckets until the instant that the electrons strike the target. If target engagement is accomplished by $\mathrm{rf}$ turn-off, much of the rf structure of the orbiting electrons is lost before they strike the target and accordingly the photon beam exhibits little rf structure.

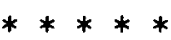

\section{Bibliography:}

T. L. Collins and J. R. Rees "Trajectories of gamma-ray beams produced by targets within the principal orbit," CEA-A-29 of $10 / 7 / 60$.

T. L. Collins, informal memorandum of about $6 / 23 / 61$ on beam-bump system.

W. A. Shurcliff, informal memorandum of $7 / 6 / 62$ on nominal paths of photon beams; $6 \mathrm{pp}$. W. A. Shurcliff and G. E. Fischer "Method of determining photon beam in straight section," CEA-TM-84 of $5 / 29 / 62$. 
Chapter 25

Synchrotron Radiation

25.1 Introduction

25.2 Predicted characteristics of the radiation

25.3 Observations of the radiation

\subsection{Introduction}

The synchrotron radiation emitted by the orbiting electrons is important in three ways. First, it constitutes the sole significant power loss by the high-energy orbiting electrons; the loss is so large, and increases so rapidly with electron energy, that it sets the practical upper limit on energy attainable in an electron accelerator of given radius. Second, it produces an anti-damping effect on the horizontal betatron oscillations of the electrons and would cause horizontal blow-up of the beam if the acceleration interval were prolonged greatly; see p. 3.14 for further details. Third, it strikes a slender strip of the vacuum chamber wall and produces some heating there; possibly it produces some radiation damage there, and perhaps it also produces some "photo-desorption" of gas molecules contained within the wall material.

\subsection{Predicted Characteristics of the Radiation}

Synchrotron radiation is electromagnetic radiation that is emitted by high-speed charged particles that are being deflected by an intense magnetic field. If the particles are electrons, if their speed exceeds about $0.999,999 \mathrm{c}$, and if they are traveling transversely to a magnetic field the magnitude of which exceeds several kilogauss, the synchrotron radiation is extremely intense and covers a broad spectral range that includes the $\mathrm{X}$-ray, ultraviolet, visual, and infra-red regions.

The principal articles on the predicted and observed characteristics of synchrotron radiation are listed in the bibliography appended to this chapter. Only a few of the characteristics will be mentioned here.

In 1949 J. Schwinger showed that, to the extent that classical electromagnetic theory is valid, the instantaneous rate $S_{e}$ at which a given electron (of charge e and rest mass $E_{o}$ ) emits synchrotron radiation varies as the fourth power of the instantaneous energy $E_{i}$ of the electron and varies inversely with the square of the instantaneous radius of curvature $r_{i}$ of the path. More exactly, 


$$
S_{e}=\frac{2 e^{2} c}{3 r_{i}^{2}}\left(\frac{E_{i}}{E_{o}}\right)^{4}
$$

To compute the amount of energy radiated by the electron in the time taken for it to travel once around the orbit, one must multiply by $2 \pi r_{i} / c$. One obtains

$$
\frac{4 \pi \mathrm{e}^{2}}{3 \mathrm{r}_{\mathrm{i}}{ }^{2}}\left(\frac{\mathrm{E}_{\mathrm{i}}}{\mathrm{E}_{\mathrm{o}}}\right)^{4} \text {. }
$$

A 6-Bev electron traveling within the gap of one of the $48 \mathrm{CEA}$ magnets follows a path having a radius of curvature $r_{i}$ of about $86 \mathrm{ft}$., and the energy it radiates per turn around the orbit is $4.5 \mathrm{Mev}$. Most of the emitted energy consists of X-radiation of 0.5 to 50 angstrom units, but even the small fraction of energy that consists of untraviolet and visible light is of high intensity. Spectral energy distribution curves are presented in the articles listed at the end of this chapter.

If the orbit were a true circle of fixed radius, the radiation would consist of a discrete set of frequencies each of which is a harmonic of the orbital frequency, i.e., an integer times 1.32 megacycles/sec. Most of the integers- would be very large, i.e., in the neighborhood of $10^{10}$ to $10^{13}$. Consequently one would not expect to be able to resolve the frequencies. Actually, electrons of slightly different momentum follow orbits of slightly different size, and none of the orbits are exactly circular; consequently the actual spectrum is reasonably continuous and smooth.

The energy radiated by a given electron at a given point on the CEA orbit is radiated in a slender cone that opens outward along the downstream tangent line to the orbit segment in question. The width of the cone of X-radiation associated with a 6-Bev electron is of the order of 0.1 milliradian. The greater the energy of the electron, the narrower the cone. The width of the cone of visibie light produced by a $\theta$-Bev electrun is of the order of 20 milliradians. As the electron travels around the orbit, the cone sweeps out a horizontal "fan."

The radiation emitted by any one electron.at any one moment is polarized. Radiation emitted horizontally by an electron traveling along an orbit that lies exactly in a horizontal plane is $100 \%$ linearly polarized with the electric vector horizontal. Radiation emitted (by such an electron) slightly obliquely to the horizontal is elliptically polarized. Since different electrons travel along paths that make different angles with the horizontal, the polarization observed along any one line of sight is a mixture. It is calculated that about $7 / 8$ of the energy radiated by $6-\mathrm{Bev}$ electrons in the CEA would pass through an ideal 
linear polarizer oriented with the transmission axis horizontal and $1 / 8$ would pass through if the polarizer were turned so that its transmission axis were vertical.

\subsection{Observations of the Radiation}

In Oct. 1962 a small, front-surface, aluminized, SiO-coated, fused quartz mirror was mounted in Straight Section Tank 26 at such an angle as to reflect synchrotron radiation (originating in the middle of the downstream half of Magnet 25) straight toward the Power Building basement via Tunnel 26. At the same time, a glass window was installed in the downstream port of that tank, to let the light out. A two-slot stroboscopic disk, rotated at speeds up to about $2000 \mathrm{rpm}$ by a G. K. Heller Co. 2T60 variable-speed electric motor, was set up in the Power Building basement, at the inner end of Tunnel 26, so as to chop the beam about 60 times a second. Adjacent to the disk, a Meischner Co. sixinch reflecting telescope was mounted so that the cross-section of the light-beam in Vacuum Chamber 25 could be viewed at a magnification of 100 to 200 times.

Results were in accord with expectations. The cross-section of the beam appeared oval; it was very bright, and somewhat bluish. As the acceleration process progressed, the oval beam became smaller and bluer. Toward the end of the acceleration interval (at about $4 \mathrm{Bev}$ ) the small dimension of the oval (the height) was a small fraction of a millimeter and the large dimension was several millimeters. At rf turn-off, a horizontal "spoke" was seen as the electrons spiraled inward in the manner indicated in Chap. 16. Many of the phenomena observed by eye were recorded by still and motion photography.

When the electron energy was below $0.2 \mathrm{Bev}$, no light was seen.

Three months later it was found that the mirror had become discolored and pitted. A new one was installed on $8 / 3 / 63$ - and was protected, when not in use, by a remotely controlled $1 / 16^{\prime \prime}$-thick stainless steel vane situated 6 " upstream. The mirror and the vane extend to within $1.8 "$ of the axis of the straight section tank.

$\underline{\text { References: }}$

\section{CEA Reports:}

K. W. Robinson "Electron radiation at $6 \mathrm{Bev}$, " CEA-14 of 10/23/56. K. W. Robinson "Radiation effects in circular electron accelerators," CEA-44 of 1/6/58. 
K. W. Robinson "Optical system for monitoring cross section of beam," CEA-TM-37 of $10 / 1 / 58$.

\section{Other Reports:}

J. Schwinger "On the classical radiation of accelerated electrons," Phys. Rev. $\underline{75}, 1912$, 1949.

D. H. Tomboulian and P. L. Hartman "Spectral and angular distribution of ultraviolet radiation from the 300-Mev Cornell synchrotron," Phys. Rev. 102, 1423, 1956.

K. W. Robinson "Radiation effects in circular electron accelerators," Phys. Rev. 111, 373, 1958.

D. H. Tomboulian and D. E. Bedo "Spectral characteristics of the radiation emitted by electrons accelerated in a synchrotron," J. Applied Physics 29, 804, 1958.

L. G. Parratt "Use of the synchrotron orbit-radiation in X-ray physics," Rev. Sci. Instr. 30, 297, 1959.

P. Joos "Measurement of the polarization of synchrotron radiation," Phys. Rev. Ltr. 4, $558,1960$.

R. Haensel "Experimente mit der Synchrotronstrahlung," DESY Report A 2.101 of August 1963. Presents superb bibliography and great variety of graphs. 


\section{Chapter 26}

\section{Cooling Plant and Water Treatment Plant}

26.1 Cooling water for the accelerator proper

26.2 Cooling water for experimentalists' magnets

26.3 Cooling tower

26.4 Water supply from the City of Cambridge

26.5 Air cooling for the accelerator proper

26.6 Air cooling for the Experimental Hall

\subsection{Cooling Water for the Accelerator Proper}

The excitation coils of the 48 magnets are cooled by "machine water." This is water that has been demineralized and has a conductivity of less than 5 micromhos; it flows in aluminum pipes that are of aluminum color; it flows in a closed circuit that includes a shell-and-tube type heat interchanger situated in the basement of the Cooling Tower.

The water is used not only to cool the copper coils of the 48 magnets but also other devices that are of copper and are isolated from the aluminum supply pipes by pieces of dielectric hose. For example, the rf cavities, the coils of the 60-ton inductor, and various power supplies in the Power Bldg. basement. This water may also be used to cool various aluminum devices.

The machine water is usually supplied at a pressure of about 80 psi and a temperature of 80 to $95^{\circ} \mathrm{F}$.

\subsection{Cooling Water for Experimentalists' Magnets}

The excitation coils of the various focusing, bending, and analyzing magnets and bubble chamber magnets used by experimentalists are cooled by "experimental water." This is water that has been demineralized and contains triethanolamine phosphate as a corrosion inhibitor; the conductivity is about 50 micromhos; it flows in green-colored steel pipes; it flows in a closed circuit that includes three heat interchangers situated in the basement of the Cooling Tower.

The water is used not only in cooling the coils of experimentalists' magnets but also for cooling other devices made of ordinary steel, stainless steel, or copper. For example: the coils of the two prototope magnets in the north corner of the Experimental Hall and the large helium compression system in the Cryogenics Room. 
The experimental water is usually supplied at a pressure of about 180 psi and about 80 to $95^{\circ} \mathrm{F}$.

(Persons designing equipment to be cooled by one of the types of recirculated water available at CEA should bear in mind the limitations as to type of metal that may be used, as well as limitations on pressure, temperature, etc. Also, they are urged to make sure that their equipment is clean before they connect it to the circulating water system.)

\subsection{Cooling Tower}

The Cooling Tower, situated beside the Power Building, employs "spray water" that extracts the heat from the heat interchangers mentioned above and delivers the heat to the ambient air. The water, treated with triethanolamine phosphate corrosion inhibitor and with household bleach to inhibit growth of algae, is pumped via blue pipes into the twocell, forced-draft, cooling tower proper. This latter is constructed of California redwood and is sheathed with aluminum. Suitable regulators are provided, and operation is largely automatic. The maximum flow rate is 4200 gallons per minute, and the total heat dissipation capability is perhaps 8 megawatts.

26.4 Water Supply from the City of Cambridge

Because the reservoirs that serve the City of Cambridge are occasionally low, the designers of CEA planned the CEA cooling system so that most of the water used is cycled in a closed system and only a relatively small amount of "new water." is needed. New water is obtained through 2-inch-diameter pipes. There are four of these available. The pressure is about 60 to $65 \mathrm{psi}$. The temperature ranges from about $40^{\circ} \mathrm{F}$ in February to about $75^{\circ} \mathrm{F}$ in August.

Some of this new water, is. used (en route to the Cooling Tower) to cool the closedcircuit water systems that cool various components of the linac.

\subsection{Air Cooling for the Accelerator Proper}

Air taken in at the NW side of the Power Building by large blowers is forced to pass downward into the basement and thence radially outward along the four radial tunnels and into the circular tunnel., It is exhausted from the tunnel by large blowers situated in the West Exit House and East Exit House. The blowers are running whenever the accelerator is running and at most other times also; thus there can be no appreciable build-up 
of ozone or radioactive gases in the circular tunnel or spur tunnel. In winter the air taken into the Power Building is heated by steam coils.

\subsection{Air Cooling for the Experimental Hall}

Outdoor air is forced into the Experimental Hall by fans and blowers situated on the mezzanine floor of that Hall. Five single-speed, axial-type 25,000 $\mathrm{cfm}$ fans are available, and six 15,000 cfm blowers. The air emerges from the Hall via louvres in the outer (southeast) wall. Because the louvres are near the ceiling and because the ceiling itself slopes upward toward the louvres, no large amount of hydrogen gas can be trapped in the upper portion of the Hall. The heat dissipation capability of this air cooling system is rated at about 3 megawatts. Associated with each of the $15,000 \mathrm{cfm}$ blowers there is a steam coil for use in heating the incoming air in winter.

References:

Chas. T. Main Co. pamphlet entitled "Cooling Systems Operations Manual." 


\title{
Tune-Up and Early Operation
}

\author{
27.1 Initial operation \\ 27.2 Tune-up \\ 27.3 Later operation
}

\subsection{Initial Operation}

After being reassembled in the Spur Tunnel, the linac was operated successfully on Aug. 1, 1961, and on Oct. 5 of that year electrons werc injected into a train of 16 of the 48 magnets. By mid-February of 1962 the 60-ton inductor of the magnet power supply had been energized at intermediate levels and assembly of the $750-\mathrm{ft}$ vacuum ring had been completed. On March 1, 1962 the synchrotron-area interlock system was ready for use, and on the following day high-level $\mathrm{rf}$ power was transmitted to the waveguide ring for the first time. On March 5 multiturn circulation of electrons in the vacuum ring was achieved with d.c. excitation of the 48 magnets. At 4:00 p.m. on March 7, 1962, the accelerator sprang into full life and became the world's highest energy accelerator; the electrons acquired energies as great as $2.2 \mathrm{Bcv}$. Operation was halted at 4:45 p.m., champagne was drunk, and telegrams were dispatched.

\section{$\underline{27.2 \cdot \text { Tune-Up }}$}

To describe the tune-up activities of the succeeding months would be difficult. A wealth of information on day to day developments is available (in the Operations Log Book, started on $3 / 6 / 62$ by $\mathrm{H}$. Winick, and in an informal seven-volume "Compendium on the CEA" by W. A. Shurcliff), but is difficult to summarize, perhaps because so few of the difficulties were difficulties of principle. The fact is that no gross errors of large stature in the design or construction came to light. Although many components were found to exhibit a variety of shortcomings, all components were operable to a considerable extent and all were capable of being improved within a few months to such an extent that the accelerator could be used routinely and reasonably reliably up to energies of several Bev and intensities of several milliamperes. Later improvements, and the attainment of the 6-Bev goal, are discussed in Section 27.3

In tuning up the accelerator, the Operations men made much use of the various indicators that had been provided, e.g., indicators of linac pulse shape, linac timing, 
inflector current, magnet excitation waveform, beam intensity, beam horizontal and vertical displacements, rf system frequency, rf modulation waveform, temperature of $\mathrm{rf}$ cavities, and gas pressure in the vacuum ring. Several other indicators were added during the first months of operation of the accelerator; these included an indicator of the temperature of the upstream post of the Y-type injection chamber, an indicator of rf reflected power waveform, and scintillation-type spill indicators.

The main goals, during the first few months of tune-up activity, were to:

1. Find how the linac beam and orbiting beam responded to the controls provided.

2. Evaluate the frequencies of horizontal and vertical betatron oscillation at injection and at high energy and find what pole-face-winding currents would correct the frequencies at injection.

3. Measure the orbit distortions that occurred at injection and find what correction coil currents would reduce the distortions to adequate extent.

4. Study the "one-and-a-half-inch effect" and see to what extent it can be overcome. (To date, the operators of the accelerator have been unable to demonstrate that any effective use can be made, at the start of the acceleration interval, of magnet-gap cross-sectional regions that lie more than about $3 / 4$ inch farther out, or more than $3 / 4$ inch farther in, than the ideal equilibrium orbit - measured at a straight section). If the attempt is made to move the equilibrium orbit (at injection) outside of the central $1 \frac{1}{2}$ inch band, or if the electrons travel outside this band because of large synchrotron oscillations: or large horizontal betatron oscillations, the electrons in question are promptly lost from the beam. On some occasions the privileged band is slightly wider than $1 \frac{1}{2}$ inches, and on some occasions it is as narrow as $3 / 4$ inch. The cause of the one-and-a-half inch effect is not known; possibly it is associated with errors of higher order components of the fields of the 48 magnets.

5. Find the optimum rf modulation waveform and explore any beam loading difficulties.

6. Maximize the beam intensity at whatever energy was chosen.

7. Find how to increase the stability of operation.

8. Find how to simplify the operating procedures so that the accelerator can be run by a small crew of men. 
During tune-up a large number of defects or other shortcomings of various components were uncovered. Much effort was devoted to eliminating the shortcomings or compensating for them. Some of the more interesting shortcomings' are listed below:

Magnets and magnet power supply: At the start of the acceleration interval, different magnets appeared to be. excited to different extents, and the excitations in successive cycles appeared to vary also. : These difficulties were probably due in large part. to insufficient regulation of the power that was supplied to Power Supply No. 1 and Power Supply No. 2 (see. Chap. 6), to insufficient filtering of 360-cycle ripple, to leakage currents in the capacitor banks, and to stray capacitances. Also, the design of the coils of the 60-ton inductor was such that, on a number: of occasions, a coil failed mechanically, perhaps because of sudden large transient currents. The tie-rods of the inductor failed occasionally, the coil clamps proved to be inadequate, the end-plates of the core blocks tended to become overheated, and the silicone-oil coolant leaked. The capacitor banks suffered from a "rainy day" effect.

$\because$ Pole face windings: Some short circuits and faulty terminals were found.

Linac: A great many components proved to have neither the stability nor the reliability needed. The time delay in initiating linac pulses. was large and the variability in delay was appreciable. It became clear, for reasons stated in Chapters 8 and 31, that the maximum energy provided by the linac $(30 \mathrm{Mev})$ is too low for really satisfactory operation of the synchrotron.

Inflector: A variety of difficulties arose.

RF system: Many minor problems arose; these included parasitic oscillations in the transmitter, non-linearity of the transmitter's response to modulation, imperfect tuning of the cavity system, leakage of $\mathrm{rf}$ power from waveguide expansion joints, failure of a waveguide switch. Beam loading appeared to contribute to the limitations on intensity of circulating beam and is a problem of continuing interest.

Vacuum chambers: Many of the chambers developed leaks for various' reasons. Chambers just downstream from the linac or from targets, scrapers, etc., suffered radiation damage; the chamber coatings became discolored and brittle, and leaks soon developed.

Vacuum pumps: Various electrical difficulties in the high-vacuum pumps proper and in the power supplies were encountered. Spontaneous out-gassing of the pumps was sometimes a problem; on a number of occasions several of the pumps began to "run away" and had to be shut off.

Monitors of beam intensity and transverse displacement: Various minor difficulties arose. 
Nearly all of these difficulties were overcome - by means discussed in the pertinent preceding chapters.

The frequencies of betatron oscillations were found to conform closely to the design frequencies, at high energy. At injection the situation was less satisfactory, presumably because of remanence in the magnets, but correction was accomplished with the aid of the quadrupole-type pole face windings, as explained in Chapters 4 and 7.

The orbit distortions at high field were found to be small. The rms value of vertical and horizontal distortions were of the order of $1 \mathrm{~mm}$ and $3 \mathrm{~mm}$ respectively. The distortions at injection were much larger, probably because the magnetic shielding against stray magnetic fields was imperfect and because of remanence in the 48 magnets; vertical and horizontal distortions as large as $7 \mathrm{~mm}$ and $25 \mathrm{~mm}$ were measured in some straight sections, when the correcting coils discussed in Chap. 15 were not in use. However, even when these coils were in use, the one-and-a-half inch effect remained.

Beam loading was found to be a problem, and tended to limit the beam intensity. The freshly injected electrons tended to induce reverse voltages (in the $16 \mathrm{rf}$ cavities) of the order of $1 \mathrm{Mev}$ per turn; coherent synchrotron oscillations of the orbiting electrons were produced, a phase shift in cavity voltage occurred, and the amount of rf reflected power increased, as explained in an informal memorandum of July 1963 by G. A. Voss. The difficulty was diminished, and the beam intensity was increased, by use of frequency modulation of the rf transmitter; see Chapter 10.

\subsection{Later Operation}

On the first day of operation (March 7, 1962) the accelerator produced electrons of 2.2 Bev energy. Two weeks later (March 21) it was operated at $3.2 \mathrm{Bev}$, but operation was halted when excessive vibration of certain components of the 60-ton inductor was noticed. In April and May 1962 the accelerator was operated with but a single rf cavity under power, to permit study of machine performance in the absence of beam loading. On May 18, 1962, after all 16 cavities had been reconnected, beam currents as great as 7 milliamperes were achieved.

On Aug. 13, 1962, after a 7-week shutdown to permit some reconstruction of the 60-ton inductor (see Chap. 6), the accelerator was operated briefly at $6.25 \mathrm{Bev}$. On Aug. 21, 1962, an external photon beam was produced by inserting a thin target in Straight Section 8; the resulting bremsstrahlung beam was allowed to enter the Experimental Hall, 
and the way was thus clear for experiments to be started. Dedication ceremonies were held on Sept. 14, 1962 .

In the following 18 months, operation. was gradually improved. Additional targets, including a small liquid hydrogen target, were installed in straight sections in the Target . Area. Additional beam runs and collimation systems were prepared and aligned, and Ex-. perimental Hall floor areas. and auxiliary. interlock systems were made: ready for use. .. A number of quantameters, ionization chambers, and Faraday cups for appraising external : beams were built. A variety of special vacuum chambers of "exit type" for use with external beams were designed and procured.

In December 1962 two-shift operation of the accelerator was begun, and on June 3, 1963, three-shift operation was started. In September 1963 the new Control Room was put to use. The beam-bump method of target engagement was put into use on April' 10, 1963. Equipment for synchronizing the linac pulses and synchrotron rf was designed, and likewise equipment for achleving momentum match between linac and synchrotron.

Meanwhile a large variety of magnets for use by experimentalists had been designed and procured, and suitable power supplies and controls had been provided. Also a large cryogenic plant was built, construction of a number of coolers for liquid hydrogen targets was begun, additional shielding blocks were purchased, radiation monitoring systems were extended, and a new Counting Room was built. Equipment for ejecting the orbiting highenergy electrons and forming an external electron beam having good geometry and long spill time was developed and tried out successfully. A positron beam, produced by a double conversion process, was made available for use by experimentalists.

In tests made on September 20,1963, electrons were successfully retained in orbit throughout six complete cycles of accelerator operation, i.e., for $0.1 \mathrm{sec}$. Suitably modified waveforms of magnet excitation and $\mathrm{rf}$ modulation were employed, and the linac and inflector were operated only ten times per second.

Currently (March 1, 1964) the accelerator is running well. Despite occasional difficulty with the linac and the 60-ton inductor, and frequent minor difficulties with other components, operation is reasonably consistent. During 3-Bev operation, intensities of 4 to 6 milliamperes are common; at 5 or $6 \mathrm{Bev}$, intensities of 2 to 4 milliamperes are not uncommon. The highest intensity achieved to date is 14 milliamperes, achieved during a 5-Bev run on April 10, 1963. 
As early as September 1962, CEA physicists put the accelerator to use in connection with shielding experiments described in Report CEAL-1004; they employed a 4-Bev photon beam in the Beam 8 area of the Experimental Hall. By Jan. 15, 1963, two groups of high-energy physicists from nearby universities were actively engaged in high-energy experiments involving electron-proton scattering and wide-anglc muon production. By March 1, 1964, eight experiments had been completed, four others were in progress, and eleven others were being prepared. 


\section{Chapter 28}

\section{Magnets for Use in Experiments}

28.1. Types of :magnets needed

28.2 Design considerations.

28.3 Design and procurement

28.4 Power supplies

28.5 Cooling

28.6 Supports

\subsection{Types of Magnets Needed}

By far the most satisfactory tool for manipulating beams of charged, high-energy particles is the electromagnet. In most applications it is far superior to electrostatic devices and to permanent magnets of comparable cost.

The main functional classes of clectromagnets used at CEA are as follows:

Clearing magnet. This is used to deflect charged particles to right or left (depending on the sign of charge) while allowing photons, and also neutral particles of all kinds, to proceed straight ahead. The typical use is to "clean up" a photon beam. The pole faces are usually parallel, and the dimension along the beampath is usually large compared to the width of the useful aperture of the gap. No great accuracy of manufacture or energizing is required.

Bending magnet (deflecting magnet, analyzing magnet). This is used to change the direction of a beam of particles having given charge and momentum and to deflect particles having different charge-to-momentum ratio through different angles so that they can be detected or used separately. The pole faces are usually parallel and long. If particles of one sign of charge only are of interest, the entire magnet may be designed to "curve with the particles." Accuracy of manufacture and energizing is usually essential.

Pair spectrometer magnet. This is designed to permit accurate separation of positively and negatively charged particles and accurate measurement of charge-to-momentum ratio of particles of either sign. Thus the pole geometry must be symmetric with respect to the axis of the incident beam, and high accuracy of manufacture and excitation is essential. In one common design the pole face is circular. Another design employs long parallel pole faces that are wider at the downstream end than at the upstream end, to allow room for particles curving to right or left. 
Quadrupole-type focusing magnet. This magnet, which possesses four symmetrically arranged poles, is capable of focusing a beam of particles (of given charge and momentum) horizontally and defocusing it vertically, or vice versa. Using two such magnets in series an investigator can produce both horizontal and vertical focusing.

Bubble-chamber magnets and spark-chamber magnets. Here the designer's intent is to provide a field of approximately uniform direction and magnitude throughout the region to be occupied by the bubble chamber or spark chamber.

\subsection{Design Considerations}

The designer of an electromagnet must take into account the magnitude and distribution of field required, the necd to avoid excessively high (saturated) fields in any portion of the iron, and the costs of the iron required and the necessary machining operations. He must design excitation coils that will be compact, rugged, adequately cooled by circulating water, and convenient to construct and install. The resistance of the coils must be matched to available power supplies and to existing ceilings on power consumption by the Laboratory as a whole. .

\subsection{Design and Procurement}

The large magnets used by experimentalists were designed at CEA. The magnet frames were made from hot-rolled billets of C-1010 low-carbon steel. The windings consist of pierced rods of high-conductivity copper. Typical values of intensity of magnetic field in the magnet gap range from 10,000 to 18,000 gauss. The magnetic efficiencies of certain types were measured and found to be in the neighborhood of 0.9 .

Table 28.1 indicates the lypes and quantities of magnets procured.

\subsection{Power Supplies}

Typical magnets are powered with 100 to 500 amperes of direct current at 200 to 450 volts. Where desired, current regulation accurate to about $0.5 \%$, or in some cases $0.1 \%$ is provided.

Power is supplied by standard power supply units rated at $36,48,75,125,250$, or $500 \mathrm{kw}$. The 36 and $48 \mathrm{kw}$ units utilize silicon rectifiers and series transistors; the higherpower units use self-saturating reactors and; silicon diode rectifiers. The supplies are located in the Power Building basement and can be controlled from there or, in some instances, from the Experimental Hall or other control area. 


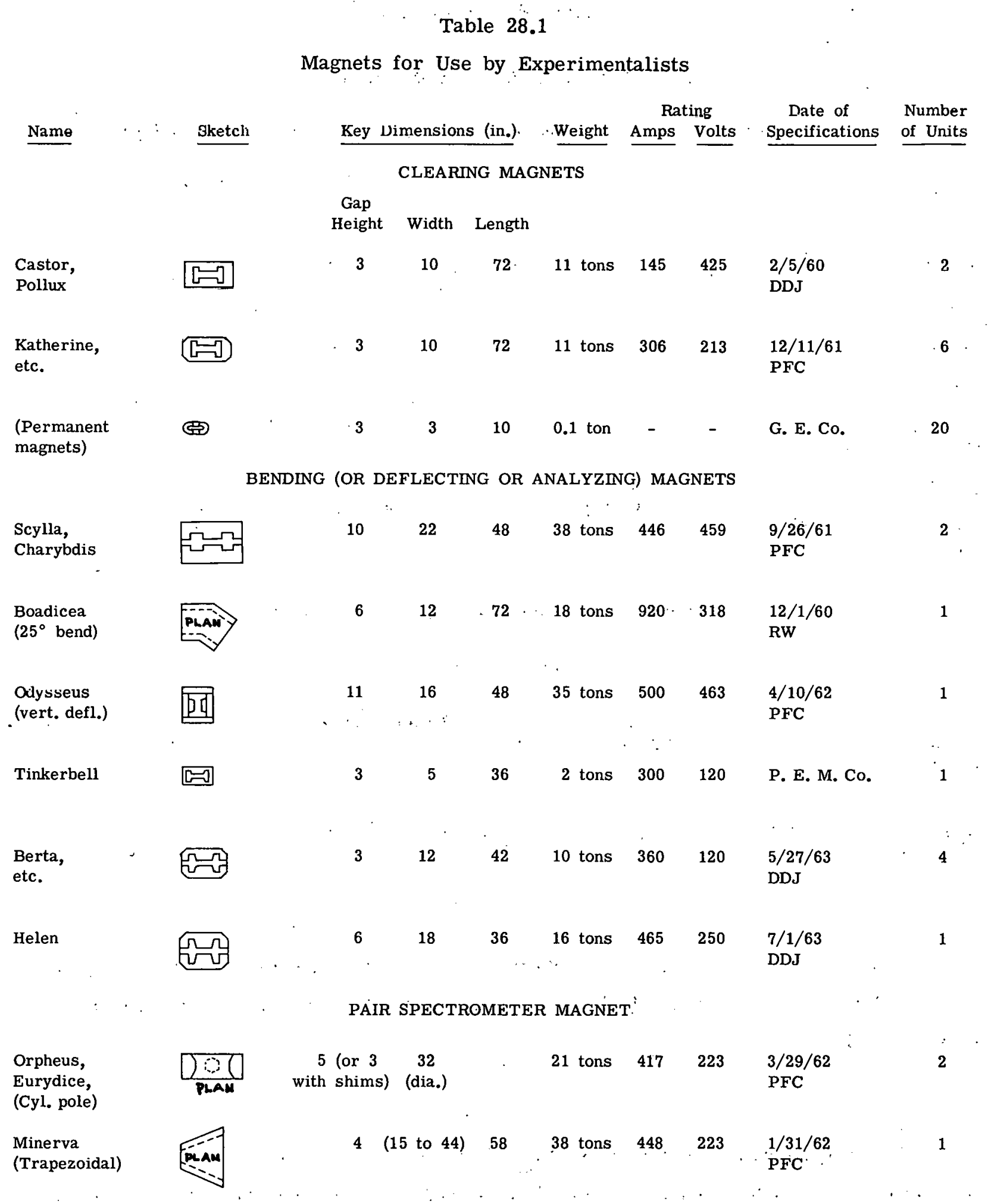


Table 28.1 (continued)

QUADRUPOLE MAGNETS (FOCUSING MAGNETS)

\begin{tabular}{|c|c|c|c|c|c|c|c|c|}
\hline \multirow[b]{2}{*}{ Name } & \multirow[b]{2}{*}{$\underline{\text { Sketch }}$} & \multirow[b]{2}{*}{ Bore (in.) } & \multirow[b]{2}{*}{ Length (in.) } & \multirow[b]{2}{*}{ Weight } & \multicolumn{2}{|c|}{ Rating } & \multirow{2}{*}{$\begin{array}{c}\begin{array}{c}\text { Date of } \\
\text { Specifications }\end{array} \\
\end{array}$} & \multirow{2}{*}{$\begin{array}{l}\text { Number } \\
\text { of Units }\end{array}$} \\
\hline & & & & & Amps & Volts & & \\
\hline $\begin{array}{l}\text { Metis; } \\
\text { etc. }\end{array}$ & $\hat{\Delta}$ & 4 & 12 & $1 / 2$ ton & 300 & 55 & P. E. M. Co. & 27 \\
\hline $\begin{array}{l}\text { Deidre,* } \\
\text { etc. }\end{array}$ & & 12 & 48 & 10 tons & 455 & 224 & $\begin{array}{l}\text { 10/24/60 } \\
\text { DDJ, PHB }\end{array}$ & 8* \\
\hline $\begin{array}{l}\text { Aideen I, } \\
\text { A ideen II, } \\
\text { (Half-quad.) }\end{array}$ & 对 & 12 & 48 & 7 tons & 455 & 112 & $\begin{array}{l}\text { 10/24/60 } \\
\text { DDJ, PHB }\end{array}$ & 2 \\
\hline $\begin{array}{l}\text { E.linor J., } \\
\text { etc. }\end{array}$ & (6) & 8 & 36 & 4 tons & 495 & 119 & $\begin{array}{l}2 / 5 / 63 \\
\text { PFC, DDJ }\end{array}$ & 4 \\
\hline $\begin{array}{l}\text { Andre, } \\
\text { Elinor R. } \\
\text { (Lozenge) }\end{array}$ & I & $(6 \times 24)$ & 36 & 8 tons & 440 & 250 & $\begin{array}{l}1 / 18 / 63 \\
\text { FPB, PFC }\end{array}$ & 2 \\
\hline
\end{tabular}

* One of these 12-inch-bore quadrupole magnets, namely Niamh, has been modified to permit extraction of a beam through the side.

OTHER TYPES

$\begin{aligned} & \begin{array}{l}\text { Colossus } \\ \text { (for bubble } \\ \text { chamber) }\end{array} \\ & \begin{array}{l}\text { Mascot } \\ \text { (for Epark } \\ \text { chamber) }\end{array}\end{aligned}$
(gap length)

The power requirements and limitations of individual magnets are indicated in a memorandum of $1 / 23 / 64$ by $W$. J. Jones. Frequency response data are presented in CEAL-TM-127.

\subsection{Cooling}

The magnet coils are cooled by water that circulates through the axial passages in the copper conductors. Chapter 26 describes the supply of "experimental water" provided for this purpose. 


\subsection{Supports}

A variety of stands are available for supporting the magnets. Most of the stands include three pedestals each of which is equipped with a precision jack to facilitate accurate positioning with respect to the incident beam.

\section{References:}

L. A. Young "Specifications for DC power supplies for experimental magnets," 6/16/60.

P. F. Cooper, Jr. "Mu-pair spectrometer design," 6/24/61.

P. F. Cooper, Jr. "Estimation of weights of deflecting magnets," CEA-TM-71 of 9/26/61.

P. F. Cooper, Jr. "Preliminary measurements on 12-inch quadrupole Deirdre," CEA-TM-74 of $12 / 11 / 61$.

P. F. Cooper, Jr., and R. Fessel "Design of an 11-inch-gap vertical bending magnet," $2 / 1 / 62$.

P. F. Cooper, Jr. "Six-inch by 24-inch luzenge quadrupole," 5/21/62.

G. E. Fischer "Emergent electron beam apparatus VII: four-inch quadrupoles," 11/13/62.

W. J. Jones "Memorandum on the powering of the individual magnets," $1 / 23 / 64$.

A. Brambilla and G. R. Gonzalez "Magnet frequency response," CEAL-TM-127 of 2/5/64.

D. D. Jacobus, P. F. Cooper, Jr., L. A. Young, P. Burr, R. Wilson: See the formal specifications referred to in Table 28.1. 


\section{Cryogenics}

\subsection{Purpose .}

29.2 Policies concerning safety

29.3 Some properties of the liquids and gases concerned

29.4 Sources of supply; quantities used

29.5 Helium compression system

29.6 Cold box for servicing the 40-inch bubble chamber

29.7 Helium liquefier

29.8 Cooler for liquid hydrogen target

29.9 Helium recovery system

29.10 Storage yard for liquid nitrogen and gaseous helium

\subsection{Purpose}

The CEA cryogenics installation was designed to serve several purposes, including these: (1) To provide low temperature helium gas for use in producing and maintaining liquid hydrogen in a 40 -inch-diameter, 550-liter-capacity, bubble chamber built by the Cambridge Bubble Chamber Group. (2) To produce liquid helium to be used as refrigerant in producing and maintaining liquid hydrogen in various small liquid-hydrogen targets. (3) To provide a supply of liquid nitrogen for use in infrared radiation shields and in the cold traps of vacuum pumping lines.

Cryogenics equipment that is designed, installed, and operated by the experimentalists themselves is outside the scope of this report. Such equipment includes the bubble cham-: bers themselves, the compressors serving the actuators (i.e., pulsers) of the bubble chambers, liquid hydrogen targets proper, and certain other devices.

\subsection{Policies Concerning Safety}

To eliminate a large fraction of the danger associated with servicing liquid hydrogen targets and liquid-hydrogen-filled bubble chambers, CEA administrators adopted the policy of using helium, rather than hydrogen, as primary refrigerant. The compressors, expansion engines, and heat exchangers associated with the expansion engines all operate on helium, not hydrogen. When a very cold liquid is to be transported within the Experimental Hall, it is helium, not hydrogen, that is transported. The liquid hydrogen used at any site is liquefied in situ with the aid of helium. 
Care is taken to see that no appreciable amount of hydrogen can escape from the bubble chamber vessels, condensation chambers, etc. To provide a margin of safety even if leakage should occur, CEA cryogenics engineers have made plans for installing devices for sensing any appreciable concentration of hydrogen in the surrounding air; if a sensor were to detect such a concentration, it would set off a local alarm. At all times powerful fans, described in Chap. 26, produce a strong circulation of air toward louvres at the upper level of the east (outer) wall of the Experimental Hall; the upward slope of the ceiling assists the elimination of air contaminated with hydrogen. At locations where controlled release of small amounts of hydrogen is to be permitted, forced-flow, flexible, 8-inch-diameter vent pipes are provided, leading outside the building.

Although the use of flames, spark-producing apparatus, open switches, etc., near quantities of liquid hydrogen is discouraged, no main reliance is placed on this approach to safety. Unavoidably, many sources of ignition are present that could trigger an explosion if a high concentration of hydrogen should occur throughout a large region.

CEA cryogenics engineers study the designs of cryogenics equipment to be procured by experimentalists, study the intended plans-of-use, and, where appropriate, suggest revisions that may increase the safety of the operation.

\subsection{Some Properties of the Liquids and Gases Concerned}

Liquid hydrogen at 1 atm. pressure boils at $20^{\circ} \mathrm{K}$ and freezes at $14^{\circ} \mathrm{K}$. The density of the liquid at $20^{\circ} \mathrm{K}$ is $4.4 \mathrm{lbs} / \mathrm{cu}$. $\mathrm{ft}$. When gaseous hydrogen is mixed with air in any proportion between 4 and 90 percent by volume and an ignition source is available, an explosion can occur. Hydrogen is cheap enough so thal it docs not have to be saved and reused, and there is no objection to venting 11 out-of doors in an approved manner. Being light, free hydrogen gas that is liberated indoors tends to collect near the ceiling and may create an explosion danger there unless adequate ventilation is provided.

Liquid helium at $1 \mathrm{~atm}$. pressure boils at $4.2^{\circ} \mathrm{K}$; at which temperature the liquid has a density of $7.9 \mathrm{lbs} / \mathrm{cu}$.ft. The heat of vaporaization of the liquid helium is low- so low that the heat necessary to vaporize one gram of the liquid is only about $10 \%$ or $20 \%$ of that necessary to raise the temperature of a gram of the gas from $4.2^{\circ} \mathrm{K}$ to $20^{\circ} \mathrm{K}$. Accordingly it is common practice, when using helium to cool other materials to temperatures as low as $20^{\circ} \mathrm{K}$, to use gaseous, rather than liquid, helium. Helium is incombustible. Because it is in short supply (controlled by the U.S. Bureau of Mines) and is expensive, effort is made to recover and re-use all appreciable quantities. 
Liquid nitrogen at 1 atm, pressure boils at $77.8^{\circ} \mathrm{K}$. It is incombustible and is sufficiently cheap that no effort need be made to recover it.

\subsection{Sources of Supply; Quantities Used}

Gaseous hydrogen, purchased from commercial distributors, is obtained in steel cylinders each of which holds 220 standard cubic feet (SCF) at a pressure of 2300 psig. The cost is about $\$ 3$ per cylinder. Expectations are that, in future months, five or ten cylinders will be purchased per week. No liquid hydrogen is procured, for reasons explained in the previous section.

The liquid helium that was used at CEA prior to March 1964 was purchased; most of it was obtained from M.I.T., in 50-liter dewar flasks. Expectations are that most of the liquid helium used at CEA in 1964 will be produced at CEA and that the production rate will occasionally reach 4000 liters per week. Perhaps $90 \%$ of the helium will be :recovered, repurified, and re-used. At any one time, as much as $\$ 5000$ worth of helium may be on hand at CEA.

Gaseous helium, purchased from commercial distributors, is obtained in 220 SCF steel cylinders; the pressure is $2300 \mathrm{psig}$, and the cost is about $\$ 9$ per cylinder. Expectations are that during 1964 about 10,000 to 30,000 SCF will be purchased per week.

The liquid nitrogen used at CEA is purchased from the Linde Division of Union Carbide Corporation. The material is brought to CEA in special tank-trucks and is stored in a 5000-liter tank described in a later paragraph. A typical shipment includes about 1500 liters. The cost is between $5 \notin$ and $10 \notin$ per liter. In mid-1963 the consumption of liquid nitrogen averaged approximately 6000 liters per week, and in 1964 the consumption is expected to be slightly greater.

\subsection{Helium Compression System}

The largest piece of cryogenic equipment at CEA is the helium compressor situated in the Cryogenics Room adjacent to the south corner of the Experimental Hall. The compression system as a whole is capable of providing 740 pounds of 550 psig, ambienttemperature, helium gas per hour. Nominally, about half of the output, i.e., 370 pounds per hour, is delivered to the helium liquefier and the remainder is available for use in. the cold box that services the 40 -inch bubble chamber.

The compressor proper, built by Ingersoll-Rand Co., includes three stages, each consisting of a double-acting reciprocating piston. The three cylinders are of progressively 
smaller size; the respective inlet pressures are 35,100 , and $250 \mathrm{psig}$ and the outlet pressures are 100, 250, and 525 psig. When an output of half or less of the maximum output is sufficient, only one half of each stage is used. Immediately ahead of each stage there is an accumulator, or cushioning tank, and immediately beyond each stage there is a water-cooled after-cooler. The last stage is followed by a mechanical oil separator and, to remove any final traces of oil, a liquid-nitrogen-cooled freeze-out trap. The compressor, driven by a 300-HP, 3-phase, $360 \mathrm{rpm}$ General Electric Co. motor, weighs 8 tons and rests on a 40-ton concrete block. Cooling is provided by a $30 \mathrm{gpm}$ flow of "experimental water" discussed in Chap. 26; the temperature of this water is slightly above room temperature.

\subsection{Cold Box for Servicing the 40-Inch Bubble Chamber}

This device, for cooling gaseous helium and supplying it to the hydrogen-recondensation coil of the 40 -inch bubble chamber, consists of a vertical cylindrical tank that contains a heat exchanger, two beds of activated charcoal, and two expanders. The tank is 48" in diameter and 130" high. Unused regions within the tank are filled with insulating powder, and the assembly is then evacuated and sealed.

The heat exchanger is of counterflow, regenerative type, and is of very intricate design. Each expander includes an expansion engine and a crosshead assembly. The piston of the expansion engine is driven by the isentropically expanding (and cooling) helium gas. Linear motion of the piston is converted to rotary motion of the crosshead shaft, and this drives a dynamotor which converts mechanical energy into electrical energy. At any one time, only one expander is in use; the other serves as standby. It is essential that there be a standby, since a prolonged breakdown of the cold bux would rosult in dumping the entire charge of liquid hydrogen from the bubble chamber.

Fig. 29.1 shows the helium flow circuit. Helium gas at high pressure and room temperature leaves the compression system and enters the warm end of the heat exchanger; as it passes along the exchanger tubes its temperature is reduced to approximately $23^{\circ} \mathrm{K}$. Still at high pressure, the gas then passes through a charcoal bed, which adsorbs small quantities of impurities, and then through the expander. Here the temperature decreases to about $13^{\circ} \mathrm{K}$ as the pressure decreases to 45 psia. The cold, low-pressure helium gas then passes through the hydrogen-recondensation coil of the bubble chamber, becoming warmed to $19^{\circ} \mathrm{K}$ while converting hydrogen gas to a liquid at $21^{\circ} \mathrm{K}$. The helium gas then 
passes upward along the low pressure leg of the heat interchanger and becomes heated to room temperature while cooling the incoming helium stream. The low-pressure roomtemperature helium gas then flows back to the helium compression system.

The cold hox was assembled, lested; and put into operation by CEA engineers. Normally, it is attached to the back of the frame of the 40-inch-bubble-chamber magnet.

\subsection{Helium Liquefier}

The helium liquefier is intended to provide a supply of liquid helium adequate for all reasonable needs by the various groups of experimentalists at CEA. It is designed to produce as much as 100 liters of liquid helium per hour, and can store as much as 1000 liters. The rate of loss of stored helium is less than 10 liters per day.

The liquefier includes three expanders arranged thermally in series, three beds of activated charcoal, and several heat exchangers, all mounted in a vertical, vacuum-jacketed, cylindrical tank. The unused regions within the tank have been filled with insulating powder, and the assembly has been evacuated and sealed. The liquid helium that is produced will be stored in a 1000-liter Dewar flask that was on order in March 1964.

\subsection{Cooler for Liquid Hydrogen Target}

The CEA has undertaken to provide semi-portable devices for cooling liquid hydrogen targets employed by experimentalists. Such a device must liquefy the appropriate amount of hydrogen at the site of use, maintain this quantity of liquid hydrogen by reflux condensation, and keep the temperature and pressure (and hence density) of the liquid hydrogen approximately constant. On $3 / 1 / 64$ just one target cooler - a prototype designed and built at CEA - was available for routine use. Expectations were that several units, built by a commercial firm, would be ready for use within a few months.

The typical cooler has the general appearance of a vertical tank about $40^{\circ}$ high and $12^{\prime \prime}$ in diameter. As indicated in the highly simplified drawing of Fig. 29.2, the outermost portion consists of a vacuum jacket; inside this there is a liquid-nitrogen-filled jacket; and inside this there is another region of vacuum. The upper, innermost region consists of a reservoir filled with liquid helium. Below, there is a hydrogen condensation chamber and a hydrogen reservoir; the former is annular and encloses the latter. The target proper is not a part of the cooler and is indicated in the diagram by dashed lines. The target is connected (by tubing) both to the hydrogen condensation chamber and to the hydrogen 
reservoir. Enclosing the target there is a copper shell which is joined (with good thermal. contact) to a copper skirt at the base of the above-mentioned liquid-nitrogen-filled jacket; thus the copper shell also is cool and serves as a radiation shield. The copper shell is enclosed in turn by a larger shell that is bolted to the base flange of the cooler proper.

The liquid helium is loaded into the target cooler manually, from a dewar flask. After enough hydrogen has been liquefied to fill the liquid hydrogen condenser, both the target and the reservoir are filled from it (by means which will not be described here). The liquid helium reservoir must be refilled at intervals of perhaps 10 to 15 hours. Each refilling requires about 16 liters of liquid helium. Suitable indicators and controllers are provided at a remotely-operated console.

\subsection{Helium Recovery System}

Recovery of a large fraction of the helium used is necessary for reasons relating to conservation and cost. In one day as much as 300 liters of liquid helium may be used; this is equivalent to 9000 standard cubic feet (SCF) of helium gas, costing about $\$ 700$.

It is expected that, during routine operation of the 40 -inch bubble chamber, associated cold-box, and compressor, no helium-recovery problem will arise: the system is a closed one, and no appreciable loss of helium, or contamination of helium, is expected.

However, loss of helium from the target coolers can occur, and opportunity for contamination can arise. Accordingly, the CEA is planning to install a header-pipe system for collecting helium from such coolers. There will be one inlet for each cooler, and at each inlet there will be a valve that is closed automatically whenever the helium vent line leading to this inlet is disconnected. The pressure in the header will be kept slightly greater than atmospheric pressure, to insure that no air leaks in. The helium gae collected will be stored in a tank discussed in the next section.

It is presumed that most of the recovered helium will be reasonably pure. If the main impurity is air that amounts to less than $0.01 \%$ by volume, purification occurs automatically during the subsequent liquefaction process. But if the concentration of air exceeds $0.01 \%$, the gas must be purified prior to liquefaction; it will be purified by being passed through a bed of activated charcoal at the temperature of liquid nitrogen.

\subsection{Storage Yard for Liquid Nitrogen and Gaseous Helium}

The Cryogenics Storage Yard, situated between the Experimental Hall and the Laboratory Building, includes the following: 
Tank for long-term storage of pure helium gas at moderate pressure; more specifically, a 400-cu.ft. tank. for storing pure helium gas at ambient temperature and a pressure of 0 to 30 psig.

Compressor ballast tank for pure helium gas at high pressure. This is a surge tank for short-term or long-term storage of as much as 400 cu.ft. of pure helium gas at room temperature and at a pressure of 0 to $550 \mathrm{psig}$. The gas is available for immediate supply to the helium compression system.

Note: The two above-mentioned tanks may be regarded as being virtually integral parts of the compression system. They are in almost constant use when that system is in operation.

Tank for storing impure helium gas. This is a $400 \mathrm{cu} . \mathrm{ft}$. tank for storing impure helium gas at ambient temperature and a pressure of 0 to 30 psig.

Tank for storing liquid nitrogen. This is a well-insulated 5000-liter tank for storing liquid nitrogen at atmospheric pressure and at about $77.3^{\circ} \mathrm{K}$. The liquid is transported to the Cryogenics Room by means of a vacuum-jacketed pipe:

Bibliography:

J. I.. Smith, Jr. "Cryogenics System for CEA," CEA-77 of 12/29/59. 


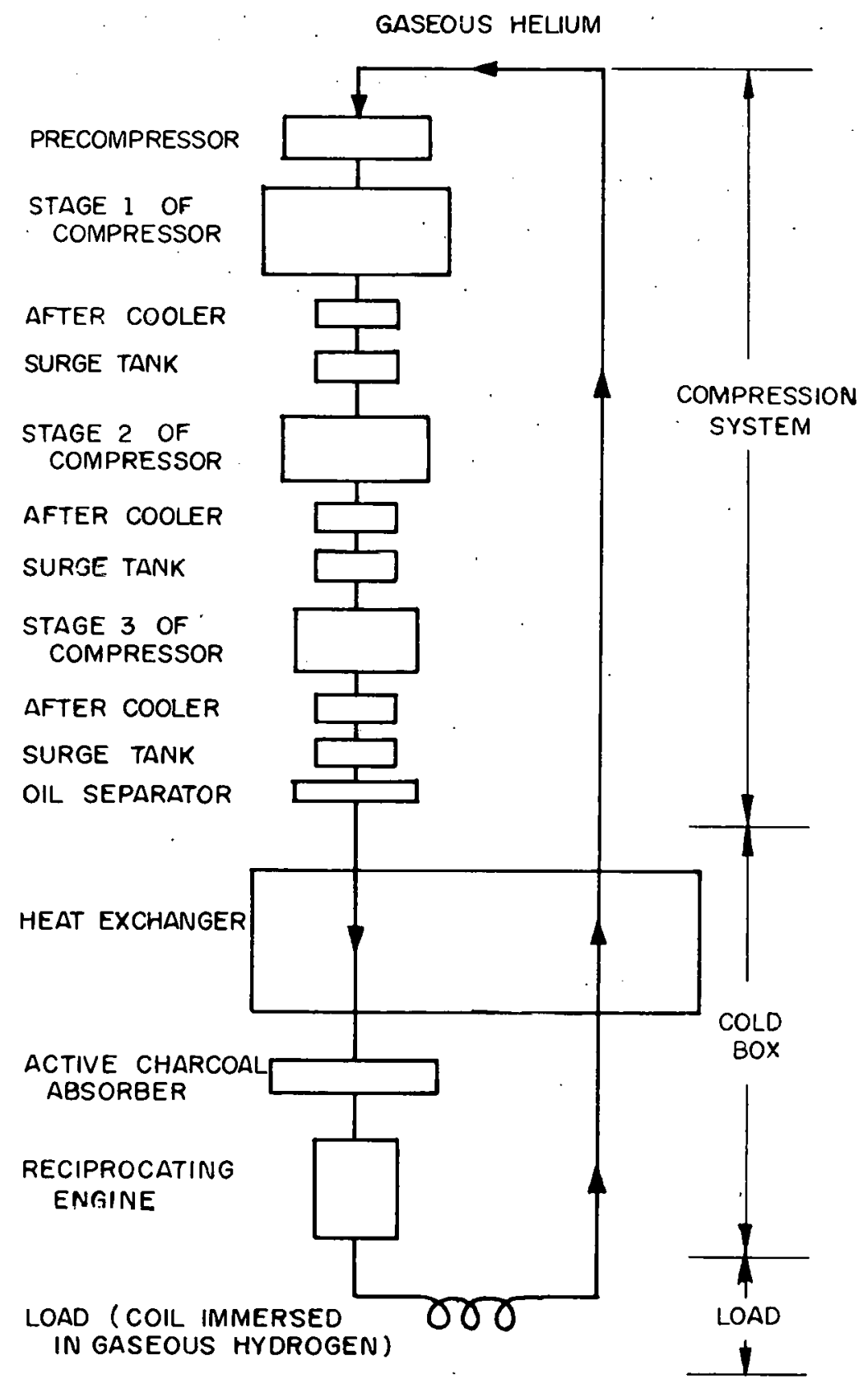

Fig. 29.1. Refrigeration system for hydrogen condenser of the 40-inch bubble chamber. The refrigerant (helium) remains in gas phase at all times. 


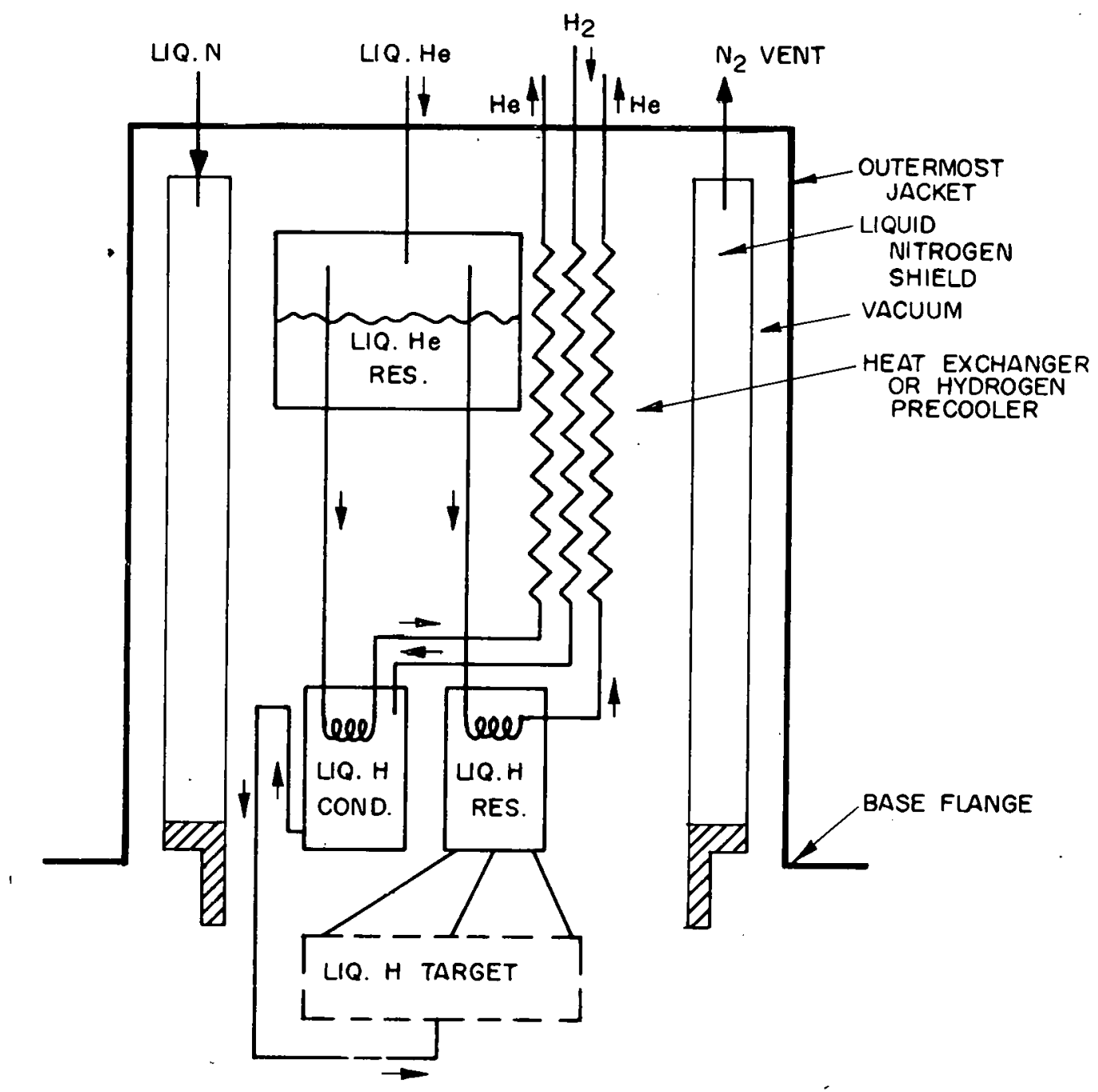

Fig. 29.2. Highly schematic diagram of a cooler for liquid hydrogen target. The liquid helium reservoir is at the top, and the liquid hydrogen condenser and liquid hydrogen reservoir are below. A vacuum-tight base housing, not shown, is bolted to the base flange, and just inside this there is a base shield, not shown, which is joined to the copper base of the liquid-nitrogen-filled infrared radiation shield. 
Chapter 30

\title{
Miscellaneous Facilities for Experiments
}

\author{
30.1 Collimators \\ 30.2 Beam transport tubes \\ 30.3 Supports for tubes \\ 30:4 Vacuum pumps for transport tubes \\ 30.5 Shutters \\ 30.6 Movable platforms \\ 30.7 Ionization chambers \\ 30.8 Quantameters \\ 30.9 Faraday cups \\ 30.10 Secondary-emission-type monitors \\ 30.11 Other detectors \\ 30.12 Conduits and cable trays
}

\subsection{Collimators}

Collimators of lead or the General Electric Co. high-density alloy Hevimet are used to reduce and sharply delimit the cross-sectional areas and angular widths of external photon beams. Hevimet, which consists mainly of tungsten (about $90 \%$ by weight) has a higher melting point than lead and also a higher thermal conductivity; in addition it can be more easily machined to close tolerances. Another advantage is its greater den-

sity, namely 17 as compared to 11.4 for lead. For Hevimet, one radiation length ( $\mathrm{X}_{\mathrm{O}}$ ) with respect to soft showers is about the same as that of tungsten, namely $0.16^{\prime \prime}$. per an informal memorandum of 1961 by F. Brasse.

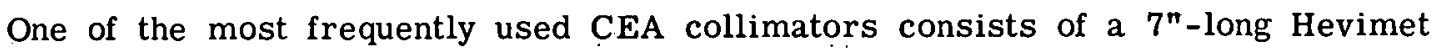
block $2^{\prime \prime} \times 2^{n}$ in cross section, with a central rectangular hole $0.125 " \times 0.330^{\prime \prime}$. The length of the block corresponds to about $43 \mathrm{x}_{\mathrm{o}}$. Hevimet collimators having other crosssections are available also. Several of the more frequently used collimators are mounted on lathe beds bolted to massive concrete blocks.

\subsection{Beam Transport Tubes}

External beams are usually housed within evacuated tubes, to eliminate unnecessary scattering. The tubes are usually of stainless steel and have outside diameters of $2 \frac{7}{8}$ inches, $3 \frac{1}{2}$ inches, or 6 inches. Tubes $2 \frac{7}{8}$ inches in diameter can be inserted between the jaws of clearing magnets having a 3-inch gap height. Tubes of greater diameter are appropriate to long unsupported runs. 
Such tubes may be joined together by means of Dresser couplings or by means of special flanges and O-rings.

\subsection{Supports for Tubes}

Most of the supports for tubes are of three-legged type and employ three-jaw or four-jaw "Steady-rest" chucks; see Drawings E-802 and D-803. A total of 76 such supports were procured. There are 10 supports of single-pillar type, described in Drawing D-1174-A.

\subsection{Vacuum Pumps for Transport Tubes}

It is customary to maintain a vacuum of about $10^{-3}$ torr or better in the tubes housing external photon beams and a vacuum somewhat better than $10^{-5}$ torr in housings that contain liquid hydrogen targets. For such purposes fore-pumps and oil diffusion pumps are used.

\subsection{Shutters}

Just upstream from each beampipe, or channel, through the Main Shielding Wall there is a remotely-controlled shutter for stopping all radiation that might otherwise pass through the hole. When the shutter is closed, persons may work in Experimental Hall areas directly downstream from the hole without danger from radiation. The shutter consists of a 16-inch-long block of lead suspended inside an air-tight evacuated housing. The lead block may be raised clear of the beam path by an electric motor controlled from the Control Room, or may be lowered into the beam. If it were to fall, it would fall into the beam path; in this respect it is fail-safe: Microswitches, connected to the appropriate Experimental-Ḧall-area linterlock system, sense whether the shutter is in open or closed position and the outcome is displayed by pilot lights in the Control Room and panel llylits in the Experimental Hall. The housing, attached to the Shielding Wall, contains flanges to which beampipes may be bolted in vacuum-tight manner.

\subsection{Movable Platforms}

A number of movable platforms, or spectrometer carriages, are used in the Target Area and Experimental Hall to support carefully aligned trains of special magnets and detectors. Usually one end of the carriage is fixed and the other end can be swung through a wide angle, on an arc-shaped track, to permit the experimentalists to obtain data pertinent 
to a wide range of scattering angles. The experimentalists' target is situated directly above the pivot point at the fixed end.

The largest of these movable platforms, MOBY DICK, was procured by CEA in 1962. The CEA is procuring another large carriage that is expected to be completed by the summer of 1964 .

\subsection{Ionization Chambers}

A number of ionization chambers for monitoring high-energy photon beams have been built and used. Some have such thin walls, e.g., walls of 1 or 2 mil stainless steel, , that they produce little scattering and could be used upstream from an experimenter's target. Others have thicker walls, e.g., 1/8"-thick aluminum walls, hence are less affected by changes in ambient pressure and produce stronger signals; however, they produce considerable scattering and are not suitable for use upstream from a target. The gas filling may consist of air, helium, or other gas.

When calibrated against an accurate quantameter and with respect to a photon beam having a given spectral energy distribution, the ionization chamber current is a measure of the intensity, or number of equivalent quanta per unit time, of an incident photon beam having this spectral energy distribution. If the spectral energy distribution is changed, a new calibration is required.

\subsection{Quantameters}

On $3 / 1 / 64$ three quantameters were available for routine use in measuring the energy of a high-energy photon beam. Several others were being completed. A typical quantameter contains a stack of eighteen 1-cm-thick copper disks separated by 1 or $2 \mathrm{~mm}$. layers of gas (such as carbon dioxide in argon, or nitrogen in helium). The stack is mounted so that the photon beam is normal to the planes of the disk. Most of the energy of the beam is dissipated in the disks, but a certain fraction goes into the production of ion pairs in the gas. The positive ions are collected, and the negative ions likewise; the total amount of positive charge collected serves as a measure of the time-integrated power of the incident beam. A precision of $\frac{1}{2} \%$ is attainable, but the absolute accuracy is usually considerably poorer. It is expected that the quantameters will be calibrated with the aid of a Faraday cup and that an absolute accuracy of 1 or $2 \%$ may then be achieved. 


\subsection{Faraday Cups}

In Jan. 1964 the construction of a 6-inch-aperture Faraday cup for determining the cumulative number of electrons in an external electron beam was completed. The cup proper, which is 12 " long and 27" in diameter, consists mainly of lead, but employs a tungsten insert and an aluminum sheath. A set of permanent magnets mounted at the front helps reduce the loss of backscattered charged particles. The space betwcen the cup proper and the aluminum housing is evacuated by means of a rough pump and a Varian high-vacuum pump. By integrating the electric current (from ground to cup) required to maintain the cup at constant potential, the operator determines the cumulative amount of charge delivered to the cup by the incident beam of elcctrons. It is hoped that an absolute accuracy of the order of $0.1 \%$ will be achicved. The device is described in CEAL-1008 of $1 / 2 / 64$ by P. H. Burr.

Some design work has been done on a much larger Faraday cup, to be embedded in the earth fill just beyond the rear wall of the Experimental Hall.

\subsection{Secondary-Emission-Type Monitors}

By $3 / 1 / 64$ the construction of a secondary-emission-type monitor was nearly complete. The device is designed to monitor the intensity of an external electron beam. The heart of the device is a series of very thin aluminum foils situated in an evacuated chamber fitted with thin aluminum windows. When the high-energy electrons strike the foils, sprays of secondary electrons are produced. These are collected, and the current they comprise is proportional to the number of high-energy electrons incident per unit time.

Because the windows and foils are so thin, the device produces relatively little scattering and therefore may be inserted tn the high-onergy electron beam at a location upstream from the experimentalist's target.

\subsection{Other Detectors}

Various other detectors of high-energy particles have been purchased or built by

CEA. One important type is the scintillation counter, which detects charged particles by virtue of "scintillation" light produced in a transparent object of appropriate chemical composition. The scintillations are measured by a photomultiplier tube.

Another type is the glass Cerenkov counter, which detects charged particles that are traveling at speeds exceeding the speed of light in the glass. When such a particle 
travels through the glass, a small amount of light is emitted and is detected by a photomultiplier.

By $3 / 1 / 64$ the construction of a magnetic spectrometer for determining the spectral energy distribution of the photons in a bremsstrahlung beam was well underway, and the device may be ready for use by 1/1/65. Photons striking a very thin target adjacent to an Orpheus-type magnet produce electron-positron pairs, which are momentum-analyzed by the 32-inch-diameter magnetic field and are detected by an array of 32 detectors each of which employs a scintillation counter and a Cerenkov counter. The spectrometer is expected to provide an energy resolution of about $1 \frac{1}{2} \%$.

\subsection{Conduits and Cable Trays}

There are 28 five-inch-I.D. conduits situated within the thick reinforced concrete floors of the Power Building basement, Target Area, and Experimental Hall. They facilitate the establishing of runs of power cables, etc., from Experimental Hall or Target Area to the Power Building basement. Early in 1961 many of the conduits were found to be partially obstructed or blocked, but by Aug. 1961 all had been cleared and made ready for use, as explained in P. F. Cooper's 7/15/61 memorandum "Conduit cleaning and testing." Various other conduits are available elsewhere; for example, there are 16 conduits following circumferential paths within the floor of the Experimental Hall and the Target Area. Different sets of man-holes provide access to the radial conduits and the circumferential conduits.

There are 28 eight-inch-I.D. conduits running radially from the Counting Room, through the massive "red wall," into the Experimental Hall.

Light-weight metal trays are provided along many of the walls of the synchrotron area and Experimental Hall for the purpose of supporting large numbers of coaxial cables and other cables. 


\section{Chapter 31}

\section{Conclusions}

The feasibility of operating a 6-Bev electron accelerator on university grounds within a large city has been demonstrated. No radiation danger to the public has arisen. Joint control of the accelerator by two universities has proved to be completely successful. Major decisions have been made in harmonious spirit by the Executive Committee drawn from the two universities, and the Joint Planning Committee has demonstrated consistent skill and tact in deciding the operating priorities from week to week.

The encouragement and financial support by the Atomic Energy Commission have been effective, and contractual and fiscal matters have been handled smoothly by the Harvard University Office for Research Contracts.

The accelerator was completed rcasonably promptly (see Chap. 2) and the final cost exceeded the estimate made five years earlier (May 1957) by only $45 \%$.

The design proved to be sound and the accelerator operates almost exactly as originally planned. The design energy is achieved, but not the design intensity. The accelerator could be made to operate with greater intensity, and also' greater reliability, if the present linac were replaced by one designed for greater stability and higher output energy; if the output energy were 60 or $70 \mathrm{Mev}$ instead of 25 to $30 \mathrm{Mev}$ as at present, the harmfulness of remanence in the magnet $\mathrm{ring}$ and of various extraneous fields would be reduced, much higher intensity could be achieved, and the usefulness of the laboratory as a whole would be significantly increased.

The close association between CEA staff and experimentalists from nearby universities has been even more rewarding than had been expected. Each group has adapted its plans promptly to accommodate the latest changes required by the other group. The accelerator is being used in a greater variety of ways, and with greater convenience, than had been contemplated; the flexibility of operation goes beyond what was contemplated when construction began.

The Operations Division is operating the accelerator effectively, and a large program of high-energy experiments is now in full swing. The backlog of experiments is large and is increasing. 
(The references are to pages, except where an entire chapter is indicated.)

Acceptance ellipse, 3.15

Administration, Chap. 2

Alarm, Chaps. 21 and 22

Alignment, Chap. 5

Alternating gradient, 3.8, 3.9, Chap. 4

Analysis, harmonic, 5.6, 15.4

Analyzing magnet, linac, 8.5

Analyzing magnet for experiments, Chap. 28

Anti-damping, 3.7, 3.14

Atomic Energy Commission, Chaps. 1 and 2

Attenuator, Venetian-blind type, 9.1

Audio intercommunication system, 23.3

Back-leg winding, Chap. 15, 16.3

Beacon, 22.2

Beam bump, 16.3, 16.4, 16.5, 27.5

Beam catcher hut, 20.2

Beam cross-section, 3.7

Beam displacement monitor, Chap. 14

Beam dumping, 23.6

Beam ejection, Chaps. 16, 17, 24

Beam intensity, 3.8

Beam intensity monitor, Chap. 14

Beam loading, 27.4

Beam path, Chap. 24

Beam pipe, 20.1, 20.2, 30.2

Beam sharing, 23.5

Bellows, Chap. 11

Bending magnet, Chap. 28

Biased waveform, 6.1, 6.2

Bremsstrahlung beam, 16.1, Chap. 24

Bubble chamber magnet, Chap. 28

Cabling, Chap. 18

Cambridge Base Grade, 3.3, 5.2, 5.3

Cambridge Electric Light Co., Chap. 18

Capacitor bank, Chap. 6, 27.3

Carriage, 30.2, 30.3

Cavity, Chap. 10

Cerenkov detector, 30.4

Characteristic length, 4.7

Choke, 60-ton, Chap. 6, Chap. 27

Chopper, linac, 9.2

Clearing magnet, Chap. 28

Closed magnet, 3.8, Chap. 4
Coaxial cable, 18.3

Coils for the 48 magnets, 4.9

Coils, orbit correction, Chap. 15

Collimator, 30.1

Concrete for shielding, Chap. 20

Conduit, 30.5

Contracts, Chaps. 1 and 2

Control Room, Chap. 19

Cooler for liquid hydrogen target, Chap. 29

Cooling system, Chaps. 26 and 29

Core block, 4.8

Correction coil, Chap. 15

Cost, Chap. 2

Counting Room, 23.10

Cradle for rf cavity, 10.15

Crash button, 22.2

Cross section of beam, 3.7

Cryogenics, Chap. 29

Cubicles for power distribution, 18.4

Current monitor, Chap. 14

Current strip, 17.2

Damping of oscillation, $3.7,3.14$

Decoupling coil, 15.4

Defocusing magnet, Chaps. 3 and 4

Design, general, Chap. 3

Diode rectifier, obsolete, 6.12

Direction of magnetic field, 6.2

Distortion of orbit, 3.14, Chap. 15, 27.4

Displacement monitor, Chap. 14

Dose, permissible, 21.1, 21.2

Dosimeter, Chap. 21

Drawings file, 23.10

Drivac pump, 12.1, 12.2, 12.3

Dry nitrogen system, 12.5

Dummy load, 10.15

Dumping system, 23.6

Ejection chamber, 11.7, 11.8

Ejection magnet, Chap. 17

Ejection of electron beam, 11.8, Chap. 17

Ejection of photon beam, 11.7, 11.8, Chap. 24

Ekco survey meter, 21.4

Electron beam, external, Chap. 17

Electron energy, Chap. 3, 23.5 
Electron speed, 3.6, 8.1

Energy demand, 3.11

Energy storage, 6.12

Equilibrium orbit, 3.9, 3.12

Equivalent quanta, number of, Chap. 20, 24.5

Executive Committee, 2.1, 2.2

Exit-type vacuum chamber, Chap. 11

Expansion engine, Chap. 29

Experimental Hall grid, 23.9

Experimental magnets, Chap. 28

External electron beam, Chap. 17, 23.7

Faraday cup, 8.5, 17.5, 30.4

Field, magnetic, Chap. 4, 6.2, 8.2

Film badge, 21.4

Financial data, Chap. 2

Flour grid, 23.9

Focal point distance, 24.2

Focusing magnets in ring, Chaps. 3 and 4

Focusing magnets for experiments, Chap. 28

FODO sequence of magnets, 4.6

Foreline assembly, 12.9

Forepump, 12.4

Frequency, linac, 8.3, 8.4, 8.5

Frequency modulation, 8.4, 8.5, 10.12

Frequency of betatron oscillation, 3.1, 3.6, $4.3,4.6,4.11,7.1,23.7,23.8,27.4$

Frequency of synchrotron oscillation, 3.10

Frequency of rf system, Chaps. 3 and 10

Frequency, orbital, 3.1, 3.3

Funds, Chap. 2

Gamma radiation, Chaps. 20, 21, 22

Gamma radiation, permissible dose, 21.1, 31.2

Gamma radiation shielding, Chap. 20

Gap height in magnet ring, 4.7

Girder, 4.8, 4.10

Gradient, 3.9, Chap. 4

Grid in Experimental Hall, 23.9

Grounding, electrical, $6.11,18.4$

Half-value thickness of ilmenite-loaded concrete, 20.5

Harmonic analysis, 5.5, 15.4

Harvard University, Chaps. 1 and 2

Health Physics, Chap. 21

Helium, Chap. 29

Height of accelerator, 3.3, 5.2, 5.3, 5.4

Height of floor, 23.9
Height survey of magnet ring, Chap. 5

Hevimet, 30.1

History of áccelerator project, Chaps. 1 and 27

Hydrogen, 23.11, 26.3, Chap. 29

Hydrogen target, 26.3, Chap. 29

Hyperbolic shape of magnet pole, 4.4, 4.5

Ilmenite, Chap. 20

Inductor, 60-ton, Chap. 6, 27.3

Inflector, Chap. 9

Injection, Chap. 9

Injection chamber, 11.6

Intensity, nominal, $3.2 ; 3.8$

Intercommunication system, 23.3

Interlock system, Chap. 22

Ionization chamber, 20.4, 21.2, 30.3

Iron-loaded concrete, Chap. 20

Isolator, $10.9,10.10$

Isomagnetic line, 3.14, Chap. 4, Fig. 13.1

Limit switch, 22.1

Linac, 3.2, Chap. 8, 27.3, Chap. 31

Linac drift tube, 9.1, 9.2, 9.3

Liquefier, Chap. 29

Liquid helium, 29.2

Liquid hydrogen, 23.10, 26.3, 27.5, 29.2

Liquid nitrogen, 29.3

Machine target, Chap. 16

Magnet, analyzing, bending, bubble chamber, clearing, Chap. 28; closed, 3.8; deflecting, Chap. 28; defocusing, 3.8; experimental, Chap. 28; focusing, open, 3.8; quadrupole, spark chamber, spectrometer, Chap. 28

Magnet coil, 4.8

Magnet core block, Chap. 4

Magnet design, Chaps. 4 and 28

Magnet end extension, 4.10, 4.11

Magnet installation, 5.3

Magnet phase angle length, 3.10

Magnet pole face profile, Chap. 4

Magnet power supply, Chap. 6, 27.3, Chap. 28

Magnet ring, Chap. 4

Magnet sequence, 4.6

Magnet steel, 4.9, 4.10

Magnet support, Chap. 6

Magnet survey, Chap. 5

Magnetic field, Chap. 4, 6.2, 8.2

Magnetic field gradient, 4.3

Magnetic inflector, 9.3, 9.4 
Magnetic shielding, 13.3, 13.4

Main parameters, 3.1, 3.2

Main Shielding Wall, 20.1

Massachusetts Institute of Technology,

Chaps. 1 and 2

Modulation waveform generator, 10.13

Momentum, Chaps. 3 and 4

Momentum compaction factor, 3.13, 4.3

Momentum match, 9.4, 9.5

Momentum vector, 3.13

Monitor of beam current, Chap. 14

Monitor of radiation, Chap. 21

Monitor of synchrotron radiation, 14.5

Monitor, TV, 23.3

Monuments, 5.2

Multiple traversal, 16.1, 16.5

n-value, 4.4, 4.5

Neutron detection, Chap. 21

Neutron shielding, Chap. 20

Neutrons, permissible dose, 21.1, 21.2

Nitrogen gas, for vacuum chambers, 12.5

Nominal intensity, 3.2

One-and-a-half-inch effect, 27.2

Open magnet, 3.4 , Chap. 4

Operation, general, 3.6, Chap. 27

Orbit correction, Chap. 15

Orbit distortion, 3.14, Chap. 15, 27.4

Orbit, equilibrium, 3.12 , Chap. 15

Orbit frequency, $3.1,3.3$

Orbit height, 3.3 .

Orbit radius, $3.1,3.2,3.3$

Orbit shape, 3.9

Oscillation: see betatron, synchrotron, parasitic

Out-gassing, Chaps. 11 and 12

Pair spectrometer magnet, Chap. 28

Parameters, main, 3.1, 3.2

Path of external electron beam, Chap. 17

Path of photon beam, Chap. 24

Parasitic oscillation, 10.11

Peaking strip, 23.1, 23.2

Peaking strip, double, 23.2

Period of grace, 22.3

Period of orbital electron, 3.1, 3.2

Permissible dose, 21.1, 21.2

Personnel, Chaps. 1 and 2

Phase angle length of magnet, 3.10
Phase angle of synchrotron, 3.11, 3.16

Phase angle of an electron, 3.16

Phase space, 3.16, 9.5

Phase stability, 3.10, 3.11

Photon beam, 16.1, Chap. 24

Pick-up coil, Chap. 14

Pier, 5.1

Planning Committee, 2.2

Platform, 30.2, 30.3

Pole face profile, Chap. 4

Pole face winding, Chap. 7

Polygon, 96-șided, 3.15, Fig. 13.1, 24.3

Power ceiling, Chap. 18

Power consumption of accelerator, Chap. 18

Power factor correction, 18.4

Power supplies for experimental magnets

Chap. 28

Power supply for ring of 48 magnets, Chaps. 6 and 27

Power Supply No. $1,6.9,6.10$

Power Supply No. 2, 6.8, 6.9

Prebuncher, linac, 8.3, 8.4, 8.5

Pump, vacuum, Chap. 12

Quadrupole magnet, Chap. 28

Quadrupole triplet, Chap. 9

Quadrupole winding, Chap. 7

Quanta, size of, 3.7

Quantameter, 20.4, 20.5, 30.3

Radial survey, Chap. 5

Radiation, gamma, neutron, etc., Chaps. 20 and 21

Radiation losses, 3.7, 10.1, Chap. 25

Radiation meter, Chap. 21

Radiation protection, Chaps. 20 and 21

Radiation safety, Chaps. 20, 21, and 22

Radio communication, 23.4

Radius of orbit, 3.1, 3.2, 3.3, 5.8

Refrigerator, Chap. 29

Remanence, Chap. 7

Repetition rate, $3.2,6.3$

rf bucket, 3.11, 3.17

rf cavity, 10.3, 10.4, 10.5, 10.14

rf system frequency, 3.2, 3.4, 10.3, 10.4, 10.12

rf isolator, 10.9, 10.10

rf modulation, 10.13

rf power requirement, $3.4,10.1,10.3$

rf system, Chap. 10 
rf transmitter, $10.10,10.11,10.12$

rf turn-off method, 10.13, 10.14, 16.6

rf waveguide, 10.7, 10.8, 10.9

Ring of magnets, Chaps. 3 and 4

S-band, 8.3

Safety, general, 23.11, 29.1, 29.2; see also Radiation safety

Scientific Subcommittee, 2.2

Seal, vacuum, 11.9

Search of tunnel, 22.3

Secondary-emission-type monitor, 30.4

Sequential beam sharing, 23.5

Shielding against gamma radiation and neutrons, Chap. 20

Shielding block, Chap. 20

Shielding, magnetic, 13.3, 13.4

Shielding, radiation, Chap. 20

Shielding wall, Chap. 20

Shutter, 30.2

Skyshine, 20.2

Sound powered interphone, 23.4

Spark chamber magnet, Chap. 28

Special access, 22.4

Spectrometer magnet, Chap. 28

Speed of electron, 3.6, 8.1

Spill indicator, 23.4

Spill-time, Chap. 16, 23.4

Stability, phase, 3.10, 3.11

Staff, 2.3, 2.4, 2.5

Steering coil, 9.1, 9.3

Stop-band, 4.3, 7.1, 17.2

Storage of energy, 6.12

Straight section, length of, 3.3, 4.5

Straight section tank, Chap. 13, 24.2, 24.3

Stray field, 8.2

Strong focusing synchrotron, 3.12, 3.17,

Chap. 4

Survey height, Chap. 5

Survey, radial, Chap. 5

Synchronizing linac and rf system, 3.5, 8.7
Synchronous phase angle of accelerator, 3.11, $3.16,10.2$

Synchronous phase angle of an electron; 3.16

Synchrotron, definition, 3.2

Synchrotron, general design, Chap. 3

Synchrotron oscillation, $3.10,3.13$

Synchrotron radiation, 3.7, 10.1, Chap. 25

Synchrotron radiation monitor, 14.5

Tank, Chap. 13

Target, Chaps. 16, 24, and 29

Target Area, 3.2, 20.1, 23.10

Telephone system, 18.3, 23.4, 23.5

Television, 23.3

Timing signal, 23.1

Transmitter, rf, 10.10, 10.11, 10.12

Tray for cables, Chap. 18, 30.5

Trigger, 23.1

TTU-1B, 10.10

Tune-up, Chap. 27

Tungsten target, Chap. 16, 30.1

Tunnel search, 22.3

Vacuum chamber, Chap. 11

Vacuum pump, Chap. 12

Vacuum requirements, $11.1,11.2$

Vacuum seal, 11.9

Valves in ring, 13.3

Venetian-blind-type attenuator, 9.1

Ventilation, Chap. 26, 29.2

VHF radin communication system, 23.4

Walkie-talkie, 23.4

Wall, shielding, Chap. 20

Warning of radiation danger, Chaps. 21 and 22

Water for cooling, Chap. 26

Waveguide for linac, $8.3,8.4$

Waveguide for $\mathrm{rf}$ system, 10.7, 10.8, 10.9

Wiring, Chap. 18 